\title{
10 Microsecond Time Resolution Studies of Cygnus X-1
}

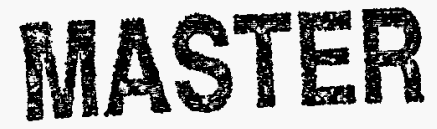

\author{
H. C. Wen \\ SLAC-Report-514 \\ June 1997

\section{DISCLAIMER}

\begin{abstract}
This report was prepared as an account of work sponsored by an agency of the United States Government. Neither the United States Government nor any agency thereof, nor any of their employees, makes any warranty, express or implied, or assumes any legal liability or responsibility for the accuracy, completeness, or usefulness of any information, apparatus, product, or process disclosed, or represents that its use would not infringe privately owned rights. Reference herein to any specific commercial product, process, or service by trade name, trademark, manufacturer, or otherwise does not necessarily constitute or imply its endorsement, recommendation, or favoring by the United States Government or any agency thereof. The views and opinions of authors expressed herein do not necessarily state or reflect those of the United States Government or any agency thereof.
\end{abstract}

Prepared for the Department of Energy under contract number DE-AC03-76SF00515 


\section{DISCLAMMER}

Portions of this document may be illegible in electronic image products. Images are produced from the best available original document. 
SLAC-R-514

UC-414

\title{
10 Microsecond Time Resolution Studies of Cygnus X-1 ${ }^{*}$
}

\author{
H. C. Wen \\ Stanford Linear Accelerator Center \\ Stanford University \\ Stanford, CA 94309
}

SLAC-Report-514

June 1997

Prepared for the Department of Energy

under contract number DE-AC03-76SF00515

Printed in the United States of America. Available from the National Technical Information Service, U.S. Department of Commerce, 5285 Port Royal Road, Springfield, VA 22161.

\footnotetext{
"Ph.D. thesis, Stanford Linear Accelerator, Stanford, CA 94309.
} 
Time variability analyses have be applied to data composed of event times of $\mathrm{X}$-rays emitted from the binary system Cygnus $\mathrm{X}-1$ to search for unique black hole signatures. The X-ray data analyzed was collected at ten microsecond time resolution or better from two instruments, the High Energy Astrophysical Observatory (HEAO) A-1 detector and the Rossi X-ray Timing Explorer (XTE) Proportional Counter Array (PCA). HEAO A-1 and RXTE/PCA collected data from 1979-79 and from 1996 on with energy sensitivity from $1-25 \mathrm{keV}$ and 2-60 keV, respectively. Variability characteristics predicted by various models of an accretion disk around a black hole have be searched for in the data. Drop-offs or quasi-periodic oscillations (QPOs) in the Fourier power spectra are expected from some of these models. A re-examination of an analysis of the Cygnus X-1 data from HEAO A-1 by Meekins, et. al. indicates that the reported excess variability at the ten millisecond time scale can be attributed to instrumental effects.

The Fourier spectral technique was applied to the HEAO A-1 and RXTE/PCA data with careful consideration given for correcting the Poisson noise floor for instrumental effects. The resulting noise-subtracted Fourier power spectrum is described by a $\sim 1 / f$ power-law with a break at $3 \mathrm{~Hz}$ in the HEAO A-1 data and between $10-20 \mathrm{~Hz}$ in the RXTE/PCA data. Evidence for a drop-off may be interpreted from the faster fall off in variability at frequencies greater than the observed breaks. Both breaks occur within the range of Keplerian frequencies associated with the inner edge radii of advectiondominated accretion disks predicted for Cyg X-1. The break between $10-20 \mathrm{~Hz}$ is also 
near the sharp rollover predicted by Nowak and Wagoner's model of accretion disk turbulence. Evidence is seen in the RXTE/PCA data for marginal excess power at frequencies from $100-4000 \mathrm{~Hz}$ with $33 \%$ of the power spectrum at those frequencies exceeding the $95 \%$ confidence level upper limit for detecting a signal in the presence of Poisson noise. No QPOs were observed in the data for quality factors $Q>9$ with a $95 \%$ confidence level upper limit for the fractional rms amplitude at $1.2 \%$ for a $16 \mathrm{M}_{\odot}$ black hole. 


\section{ACKNOWLEDGMENTS}

I wish to foremost thank my advisor, Elliott Bloom for his continued guidance, support and encouragement over the years that I have been at SLAC. Special thanks go to Professor Bloom and Professor Minh Duong-van for their efforts that have helped me succeed in continuing my graduate studies at Stanford University. The enjoyment I have received from the success of the Group K High Energy Astrophysics program derived principally from Professor Bloom's pioneering efforts. His enthusiasm for physics coupled with a grasp of important issues are ever present sources of inspiration.

Another integral part of my graduate research was the tireless assistance received by many members of SLAC for which I owe much gratitude. For teaching me experimental physics and the finer aspects of data analysis, I thank Gary Godfrey, Bill Atwood, Art Snyder and Ken Fairfield. I thank Dr. Godfrey, John Broeder, Noad Shapiro and Charles Martell for their assistance with the X-Ray Cannon experiment. Additional appreciation goes to Steve Meyer for PC consulting, Linda Lee Evans for paychecks and travel, and Terry Anderson for plots and posters.

All of the data analyzed in this dissertation were made available by Kent Wood and his group at NRL. I thank Kent Wood, Daryl Yentis, Terry Crandall and Doug McNutt for their extensive archaeological assistance in resurrecting the HEAO A-1 HighBit-Rate (HBR) data and understanding its data format. I am also grateful to Paul Hertz for the RXTE data and the sanity checks it provided against the many unique "features" I uncovered in the HBR data.

Providing experience in analyzing X-Ray data and understanding astrophysics was Lynn Cominsky (Sonoma State) and Jeffrey Scargle (NASA/Ames). I thank them both for providing insights in analyzing the HEAO A-1 and RXTE data and educating me on astrophysics. I also thank Robert Wagoner (Stanford University) for discussions about diskoseismology. 
Of course, another important element to surviving the graduate experience was the collection of friends I have made at Stanford. I thank John Hanson for his friendship and camaraderie during the many trips to NRL and Rockwell as USA was being designed, constructed and tested. When I was mired in mathematical details or befuddled with my computer, Andrew Lee has been there to lend a hand. I thank him for his generosity and our shared hiking adventures in Aspen. For her work with USA preflight data and an entertaining exchange of American and Russian cultures, I thank Ganya Shabad and hope she remembers her colleagues as she follows in her father's political footsteps. I thank Chris Chaput for helping me lose a little around my equator while I repeatedly slam dunked over him.

Finally, I wish to thank my family and most importantly express my gratitude and love for my spouse, Lavonne. She has provided unwavering support, encouragement and tremendous patience over the years I have been at Stanford. My bulging equator and good spirits are tributes to her love. Another wave of thanks must be given to my parents, Chi-Yu Hu and John Lung Wen, my parent-in-laws, Lilan and Henry Fan and KungHsung Wu, and other family members, Anna, John, Brian and Michelle. You have all helped Lavonne and I make graduate life as palatable as possible with your tremendous financial support and encouragement. 


\section{Table of Contents}

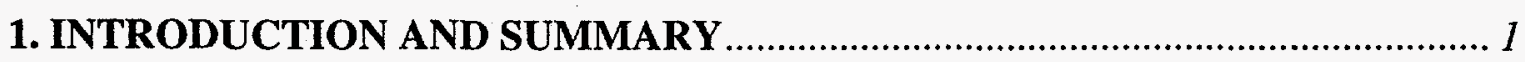

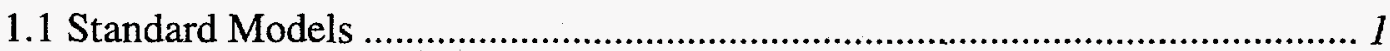

1.2 Searching for Black Hole Signatures in Cygnus X-1 .............................. 14

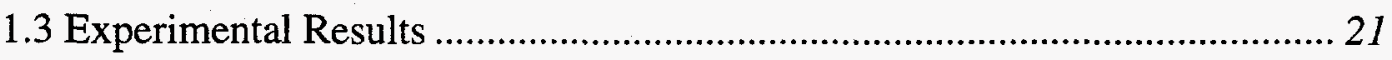

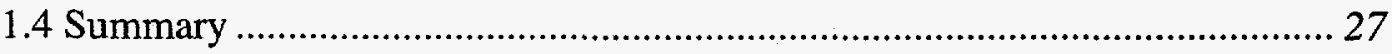

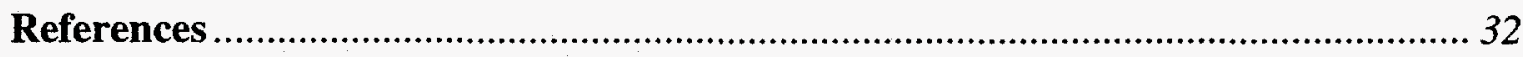

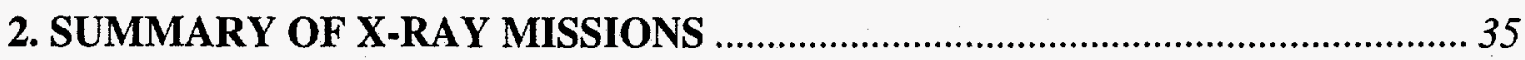

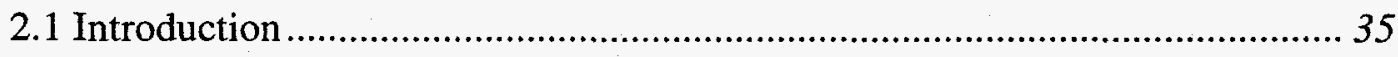

2.2 High Energy Astronomical Observatory 1 (HEAO 1) ................................ 35

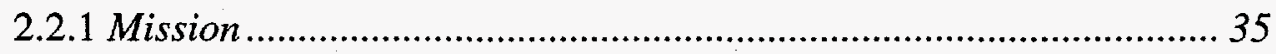

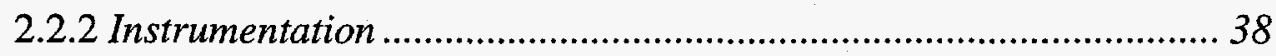

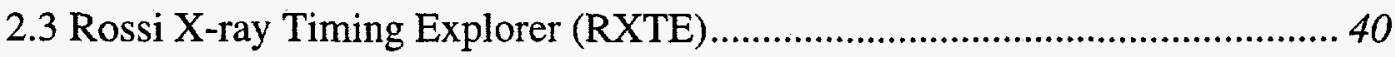

2.3.1 Mission ................................................................................ 40

2.3.2 Instrumentation ............................................................... 41 
2.4 Unconventional Stellar Aspect (USA) ................................................. 44

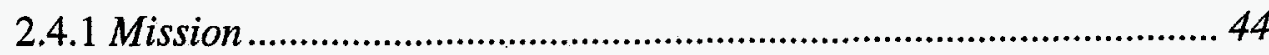

2.4.2 Instrumentation ............................................................... 45

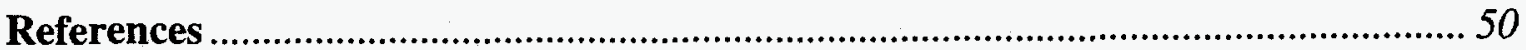

3. HEAO A-1 HIGH BIT RATE DATA STUDIES ......................................... 51

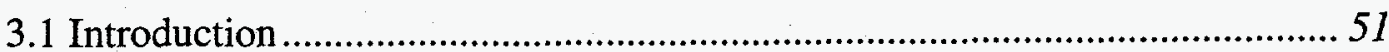

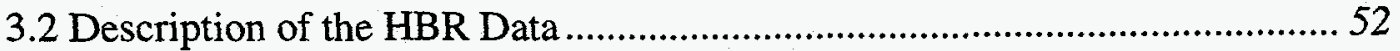

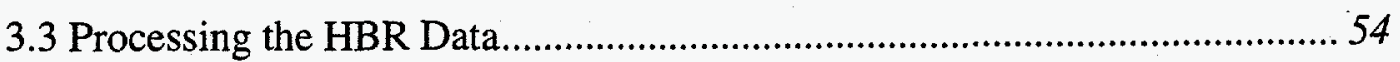

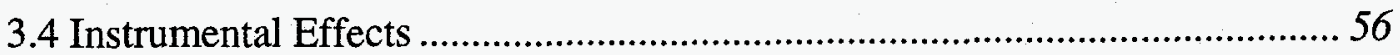

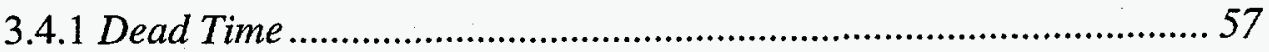

3.4.2 HEAO A-1 Module Electronics Malfunction ................................ 59

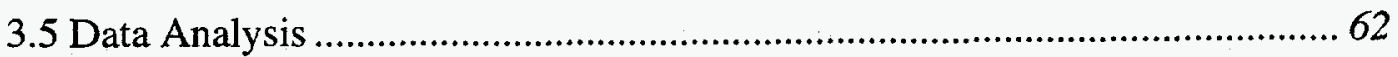

3.5.1 Correcting the Poisson Noise Floor .........................................6 62

3.5.2 Correcting the Meekins, et. al. Analysis ....................................... 64

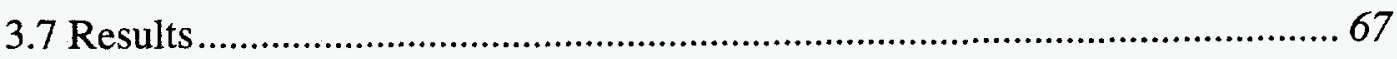

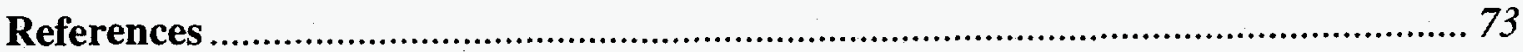

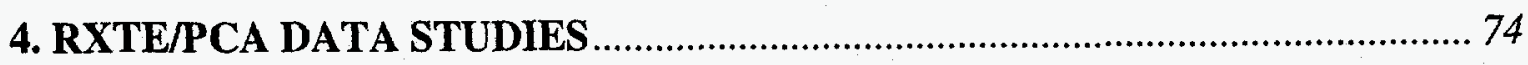

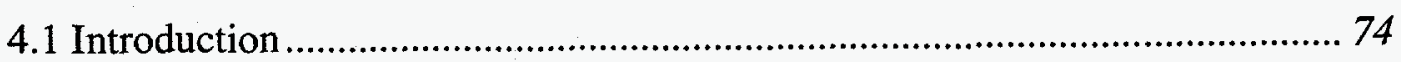

4.2 Description of the RXTE/PCA Data ................................................... 74

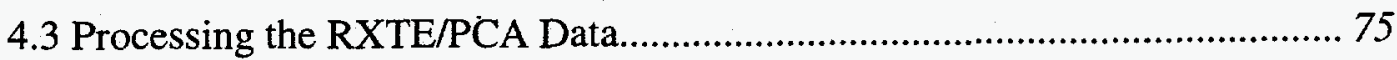

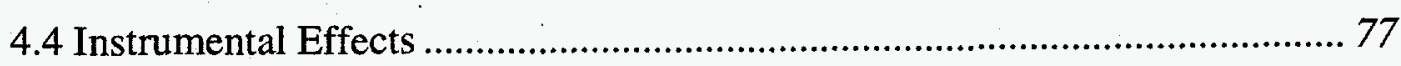




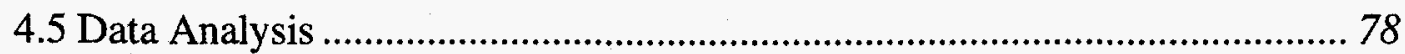

4.5.1 Correcting the Poisson Noise Floor ............................................... 78

4.5.2 Frequency Upper Limit on Detected Intrinsic Variability............... 79

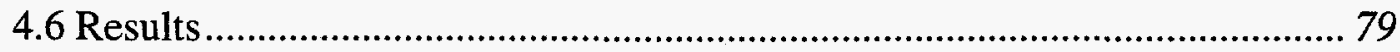

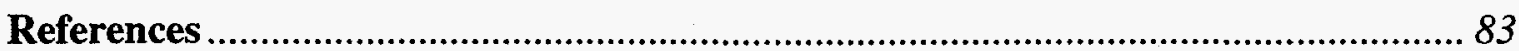

Appendix A. HEAO A-1 High-Bit-Rate Data Format ............................................ 84

A.1 Bit Telemetry Mode Format........................................................................... 84

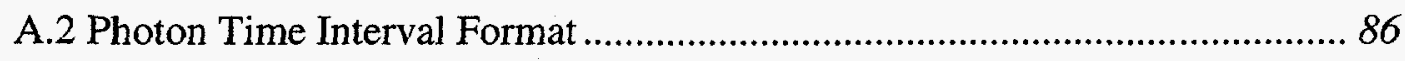

A. 3 Concatenated Time Interval Format........................................................ 92

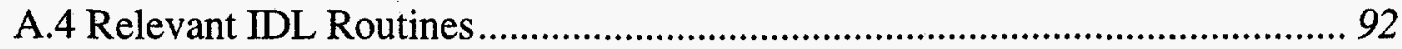

A.4.1 Bit Telemetry Mode Format ....................................................... 92

A.4.2 Photon Time Interval Format ..................................................... 100

A.4.3 Concatenated Time Interval Format .......................................... 111

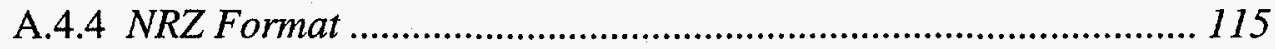

Appendix B. HEAO A-1 High Bit Rate Data Archive .......................................... 122

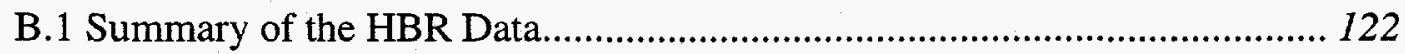

B.2 Data Screening ..................................................................................... 125

B.2.1 Correcting for Adjacent Pairs ................................................... 125

B.2.2 Data Integrity Check ……………............................................ 127

Appendix C. Dead-Time-Distorted Waiting Time Distributions.............................. 129

C.1 Probability Distribution of Binned Waiting Times .................................... 129 
C.2 Waiting Time Density of the USA Perimeter Veto Problem 130

Appendix D. Testing of the USA Collimators ............................................... 133

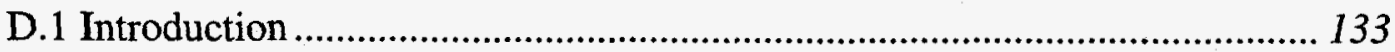

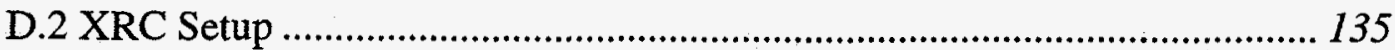

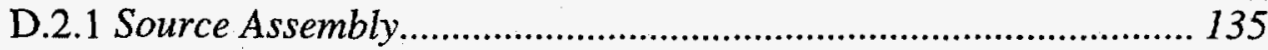

D.2.2 Detector Assembly............................................................... 137

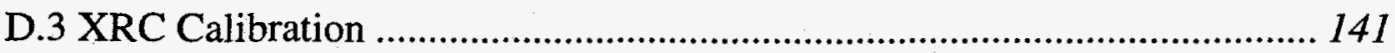

D.3.1 Alignment of the XRC Cannon ................................................. 141

D.3.2 Calibration of the Goniometer ................................................. 142

D.3.3 Plateauing of the Detector ................................................... 142

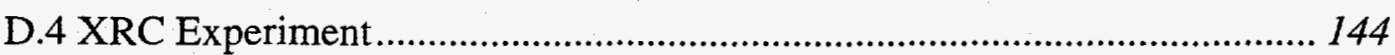

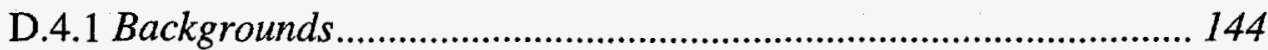

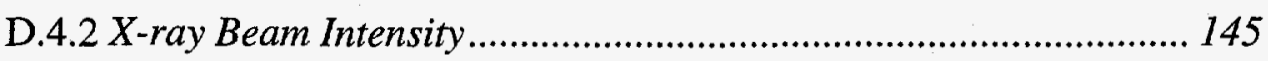

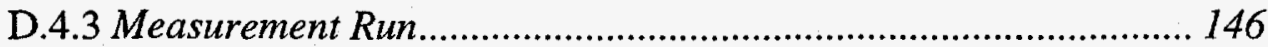

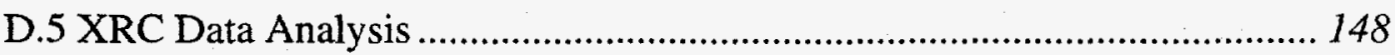

D.5.1 Coordinate Systems .............................................................. 148

D.5.2 Method for Determining Collimator Response Characteristics ... 149

D.5.3 Accuracy of the Detector Apparatus ......................................... 150

D.5.4 Determination of the Goniometer Axis ..................................... 151

D.5.5 Monte Carlo Simulation ......................................................... 153 


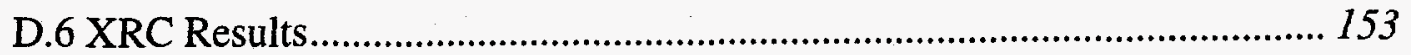

D.6.1 Characterization of the Collimator Modules ............................ 153

D.6.2 Final Selection of Collimator Modules ................................... 154

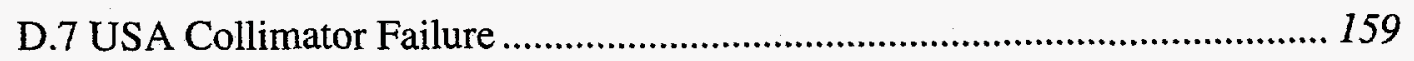

Appendix E. Charged Particle Backgrounds in the USA Detector ..................... 177

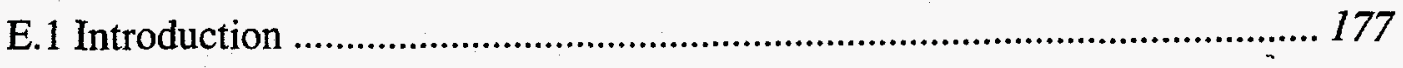

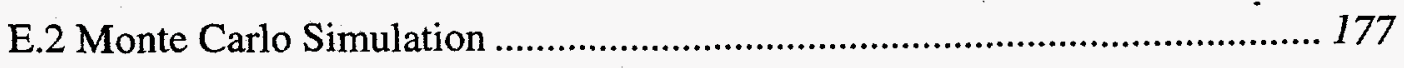

E.2.1 Sources of Charged Particle Backgrounds .............................. 179

E.2.2 Detector physics ............................................................. 180

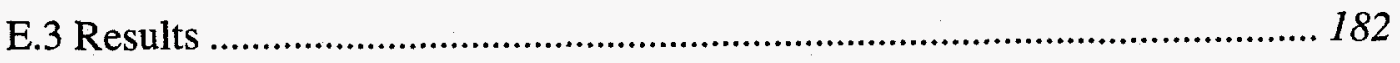

E.3.1 Charged Particle Background Counting Rates ......................... 182

E.3.2 X-ray Observation Uptime.................................................. 183 
[1.1] Illustration of a close binary system (not to scale) .......................................... 2

[1.2] X-ray energy spectra of Cyg X-1 in the high state and the low state .................... 4

[1.3] The effective potential for a test particle of mass $m$ of various angular momenta, orbiting about a Schwarzschild black hole........................... 6

[1.4] The radial distribution of the integrated flux emitted from the faces of a thin, Keplerian accretion disk ................................................................ 8

[1.5] Schematic diagram of the two component advective disk model ....................... 10

[1.6] Variation of the energy spectral index $\alpha$ for the TCAF model as functions of the disk and halo accretion rates

[1.7] Power spectrum of many simulated optically thick blobs orbiting about a black hole 12

[1.8] Combination of viscous/thermal timescale and acoustic mode power from a model of accretion disk turbulence.

[1.9] The dependence of the Keplerian frequency at the marginally stable circular orbit on the black hole angular momentum parameter

[1.10] The dependence of the frequency of the lowest radial $(m=0)$ $\mathrm{g}$-modes on the black hole angular momentum parameter

[1.11] Summary of the frequencies where drop-offs or QPOs are predicted in Cyg X-1

[1.12] The relative integral power defined by Meekins, et. al. for Cyg X-1, uncorrected and corrected for instrumental effects.

[1.13] The noise-subtracted Fourier power spectrum of Cygnus X-1 from the HEAO A-1 data. 
[1.14] The noise-subtracted Fourier power spectrum of Cygnus $X-1$ from the RXTE/PCA data.

[1.15] The 95\% confidence level upper limit for detecting QPOs in Cyg $X-1$ with a quality factor, $Q>9$ for a given relative amplitude

[1.16] The noise-subtracted Fourier power spectra of Cygnus X-1 from the HEAO A-1 and RXTE/PCA data.

[1.17] Total integrated RXTE/PCA observation time required to detect diskoseismic QPOs in Cyg X-1.

[2.1] The High Energy Astrophysical Observatory-1 HEAO-1 satellite shown with its four major experiements identified.

[2.2] HEAO A-1 effective area for a single Module $(1,2,3$ or 4$)$ for the sum of both wire layers as a function of photon energy

[2.3] The Rossi X-Ray Timing Explorer satellite......................................................... 41

[2.4] Layout and cross section of the Proportional Counter Array (PCA) ..................... 42

[2.5] RXTE/PCA effective area for all PCUs and all anodes as a function of photon energy

[2.6] The Advanced Research and Global Observation (ARGOS) satellite.

[2.7] USA effective area for both detector units and all three wire layers as a function of photon energy

[2.8] Layout of a USA detector unit with an exploded view of the collimator.

[2.9] Front views of the Unconventional Stellar Aspect Experiment........................... 47

[2.10] Rear views of the Unconventional Stellar Aspect Experiment.............................. 48

[3.1] Typical light curve for a HBR raw data file....................................................... 55

[3.2] The Fourier power spectrum and the waiting time distribution of a Poisson source, Cas A 60

[3.3] The average relative integral power for Cygnus X-1 from Meekins, et. al. and our reproduction of their analysis.

[3.4] The average relative integral power for Cyg X-1 calculated by using Leahy normalized Fourier power.

[3.5] The average relative integral power for Cyg X-1 after the Poisson noise floor is corrected for instrumental effects. 
[3.6] The raw and noise-subtracted Fourier power spectrum of Cyg X-1 from the HBR data

[3.7] The 95\% confidence level upper limit for detecting QPOs in Cyg $\mathrm{X}-1$ from HEAO A-1 with a quality factor, $\mathrm{Q}>9$

[4.1] The Fourier power spectrum of the RXTE/PCA data on the Crab, excluding values at the harmonics of the Crab pulsar frequency.

[4.2] The raw and noise-subtracted Fourier power spectrum of Cyg $\mathrm{X}-1$ from the RXTE/PCA data.

[4.3] The 95\% confidence level upper limit for detecting QPOs in Cyg $\mathrm{X}-1$ from $\mathrm{RXTE} / \mathrm{PCA}$ with a quality factor, $\mathrm{Q}>9$.

[A.1] Example of two MNF sections in the an HBR Data block 89

[B.1] Waiting time distribution for an uncorrected HBR data file showing an anomalous number of pairs of events with a waiting time of 1 .

[B.2] The average of the absolute valued differences between the corrected HBR and NRZ time series as a function of offset

[B.3] The difference between the $5 \mathrm{~ms}$ resolution time series of the corrected HBR and NRZ data for the data set, 015_2_3.

[D.1] One of three USA collimators constructed at SLAC ................................... 134

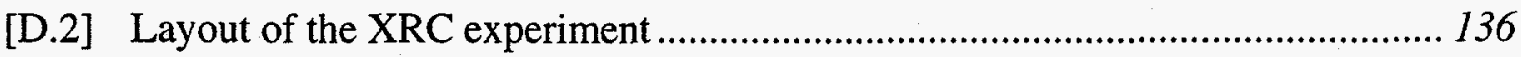

[D.3] Geometry of the XRC goniometer .......................................................... 138

[D.4] Circuit diagram of the pre-amplifier used in the XRC electronics ................... 140

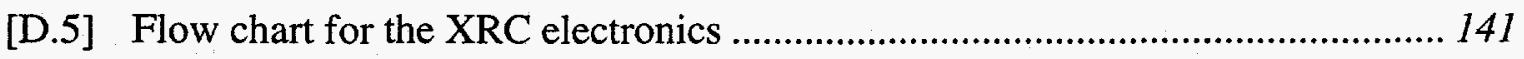

[D.6] Plateau curves for phototubes 1 and 2 and PWC....................................... 143

[D.7] Definition of $\phi$ and $\theta$ in the xyz-coordinate system ................................... 148

[D.8] Simulated collimator response functions for collimators C-1, C-2 and C-3 from data taken in XRC test

[D.9] Damage from burst PWC window behind USA collimator C-2 resulting in a large eye-shaped opening

[D.10] Scans in $\phi$ and $\theta$ for unit: Collimator C-1, Segment 1, Module 5

[D.11] Scans in $\phi$ and $\theta$ for unit: Collimator C-1, Segment 1, Module 20 ................... 162

[D.12] Scans in $\phi$ and $\theta$ for unit: Collimator C-1, Segment 1, Module 8 .................... 163 
[D.13] Scans in $\phi$ and $\theta$ for unit: Collimator C-1, Segment 1, Module 14 .................. 164

[D.14] Scans in $\phi$ and $\theta$ for unit: Collimator C-1, Segment 2, Module 1 .................... 165

[D.15] Scans in $\phi$ and $\theta$ for unit: Collimator C-1, Segment 2, Module 7 ................... 166

[D.16] Scans in $\phi$ and $\theta$ for unit: Collimator C-1, Segment 2, Module 17 .................. 167

[D.17] Scans in $\phi$ and $\theta$ for unit: Collimator C-1, Segment 2, Module 26 .................. 168

[D.18] Scans in $\phi$ and $\theta$ for unit: Collimator C-3, Segment 1, Module 2 .................... 169

[D.19] Scans in $\phi$ and $\theta$ for unit: Collimator C-3, Segment 1, Module 13 .................. 170

[D.20] Scans in $\phi$ and $\theta$ for unit: Collimator C-3, Segment 1, Module 23 ................. 171

[D.21] Scans in $\phi$ and $\theta$ for unit: Collimator C-3, Segment 1, Module 27 ................. 172

[D.22] Scans in $\phi$ and $\theta$ for unit: Collimator C-3, Segment 2, Module 11 ................. 173

[D.23] Scans in $\phi$ and $\theta$ for unit: Collimator C-3, Segment 2, Module 16 ................. 174

[D.24] Scans in $\phi$ and $\theta$ for unit: Collimator C-3, Segment 2, Module 21 .................. 175

[D.25] Scans in $\phi$ and $\theta$ for unit: Collimator C-3, Segment 2, Module 25 .................. 176

[E.1] Typical differential flux distribution of electrons trapped in the van Allen radiation belts ...................................................................................... 179

[E.2] Typical differential flux distribution of galactic cosmic rays. ......................... 181

[E.3] Transmission probabilities through the various faces of the collimator and the proportional chamber walls....

[E.4] Contour map of the expected background rates in one USA detector unit due to trapped charged particles.

[E.5] Contour map of the expected background rates in one USA detector unit due to galactic cosmic rays

[E.6] Contour map of the expected background rates in one USA detector unit due to all charged particles. 
[1.1] Summary of power law fits to the noise-subtracted Fourier power spectrum.

[2.1] Brief summary of the four experiments in HEAO 1 ...........................................37

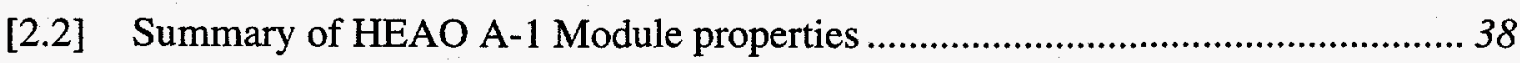

[3.1] Description of the telemetry modes available for the HBR data...........................52

[3.2] The major data processing steps for creating the HBR data archive..................... 54

[3.3] Summary of the properties of two types of dead time ….................................... 57

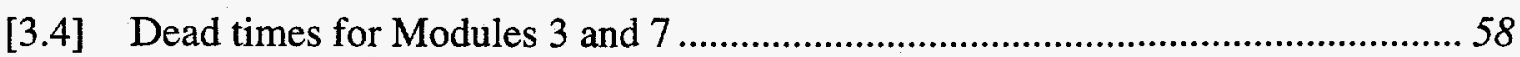

[3.5] The offset hyperexponential distribution parameters that defined the effective Poisson noise floor for two Cyg X-1 data files .................................... 63

[3.6] Summary of power law fits to the noise-subtracted Fourier power

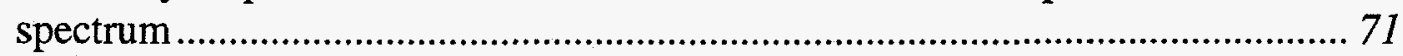

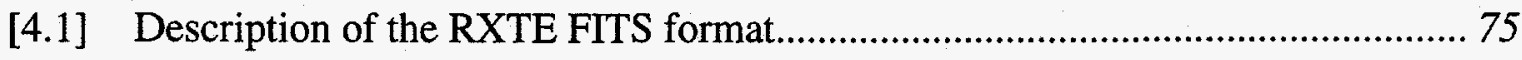

[4.2] The major off-line processing steps for the RXTE/PCA data............................... 76

[4.3] Non-extended dead times for each PCU ............................................................ 77

[4.4] Summary of power law fits to the noise-subtracted Fourier power

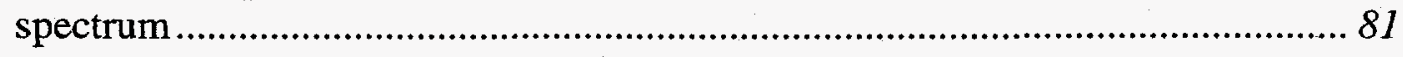

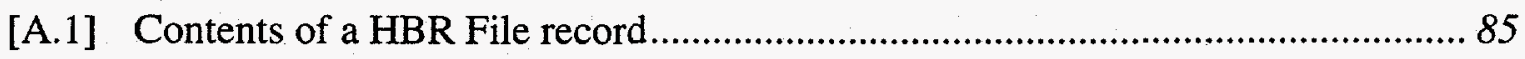

[A.2] Format of the bits in a give HBR data word ........................................................ 85

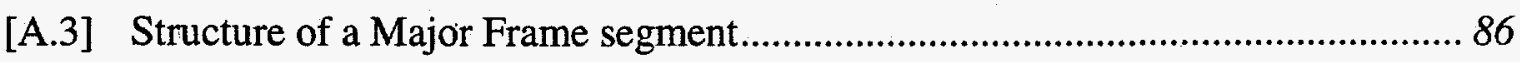

[A.4] Description of the Major Frame Header ............................................................ 87 
[A.5] Bit assignments for the MJF flags word. ............................................... 88

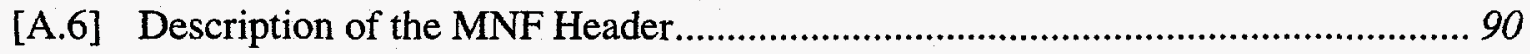

[A.7] Bit assignments for the MNF flags word ................................................... 90

[A.8] Description of the cat structure variable...................................................... 91

[B.1] Description of the column headings in Table B.2 ........................................ 123

[B.2] Summary of all usable HBR data files.................................................. 124

[D.1] Summary of the transmissions through the various materials and obstructions in the PWC ...................................................................... 146

[D.2] List of measurements made for each run ................................................ 147

[D.3] Summary of the fit results to five separate measurement runs with Collimator 18

[D.4] Euler angles needed to rotate goniometer axis to the direction observed from from the inertial $\mathrm{z}$-axis.

[D.5] Characterization of all the collimator modules by fitting an elliptical cone to the data

[D.6] Collimator modules selected for the three assembled full collimators.

[D.7] Results of fitting the simulations of the response functions of collimators C-1, C-2 and C-3 for a flat top.

[E.1] Average expected counting rate in a USA detector unit from all estimated particle backgrounds.

[E.2] The percentage of uptime below a $10^{4}$ counts $\mathrm{sec}^{-1}$ cutoff for several scenarios in the charged particle models 


\subsection{Standard Models}

Over the last three decades, the field of X-ray astronomy has yielded significant advances in understanding the end states of stars. Central to this progress was the observation and study of close binary systems in which a compact object orbits tightly about a companion star. The compact objects are believed to be stars that have reached the end of their lives or end states. By observing how a companion star's behavior is affected by the compact object, properties of the compact object can be indirectly determined. Measurements of the orbital motion of the companion star about the compact object lead to a lower limit for the compact object's mass. One type of compact object, a black hole, is by itself observationally invisible. Indirect observation is the only means whereby information about the object may be extracted.

In addition to affecting the companion's orbital motion, the compact object also strips the companion of its matter. The matter, with a specific angular momentum equal to that of the companion, gradually falls towards the compact object as the angular momentum is removed by viscosity. The flow encircles the compact primary and forms a quasi-stationary structure around it commonly known as an accretion disk, as illustrated in Figure 1.1. The process of accreting matter onto the compact object generates observable X-ray emissions. 


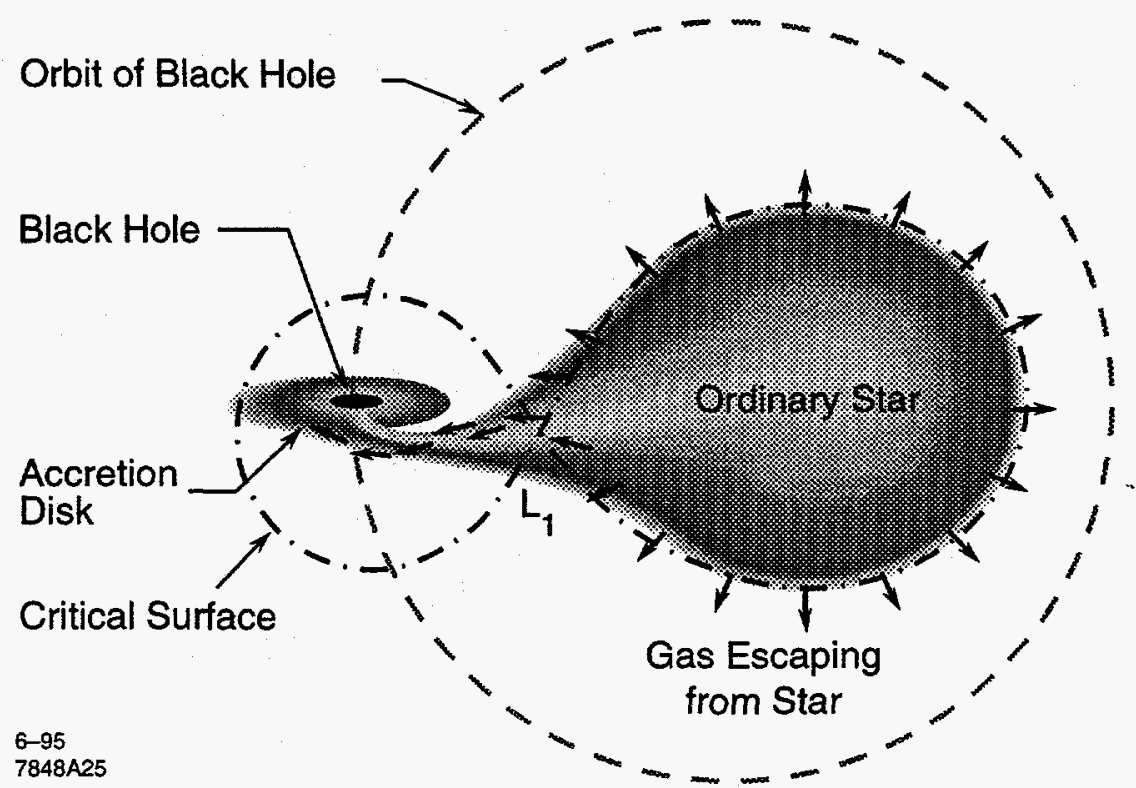

Fig. 1.1 Illustration of a close binary system (not to scale). The critical surface is the innermost self-intersecting equipotential surface commonly referred to as the Roche lobe. The compact object accretes matter that has overflowed the companion's Roche lobe through the Lagrange point, $\mathrm{L}_{1}$ and onto its accretion disk.

In our current standard model, three types of compact objects are believed to exist in close binary systems. All three compact objects are gravitationally bound. When a star has exhausted its nuclear fuel, the loss of thermal pressure leads to the star's gravitational collapse. One of the possible outcomes of the remaining core is a white dwarf with typical radii of $10^{-2} \mathrm{R}_{\odot}$ and densities of $10^{6} \mathrm{~g} \mathrm{~cm}^{-3}$, where $\mathrm{R}_{\odot}$ is the radius of the sun. For white dwarfs, the Fermi electron-degeneracy pressure balances the object's selfgravitation.

Above $10^{7} \mathrm{~g} \mathrm{~cm}^{-3}$, the density is high enough that all the Fermi levels are filled beyond the energy of the electrons from neutron-beta decay, and inverse beta decay is favored,

$$
e^{-}+p \rightarrow n+v
$$

When the density exceeds $10^{11} \mathrm{~g} \mathrm{~cm}^{-3}$ the neutron population begins to dominate both in number and in its contribution to the total pressure by its Fermi degeneracy pressure. The 
compact object at such densities is called a neutron star with typical radii of $10^{-5} R_{\odot}$.

At nuclear densities $\left(10^{14} \mathrm{~g} \mathrm{~cm}^{-3}\right)$ and higher the equation of state for matter is incomplete. Beyond a certain density no known repulsive force of nature can balance the object's tremendous gravitational attraction. It is currently believed that the remaining core continues to collapse to a gravitational singularity called a black hole, one of the most startling predictions of general relativity. The exact density separating a neutron star and a black hole is not known, but should be less than $10^{16}\left(\mathrm{M}_{\odot} \mathrm{M}\right)^{2} \mathrm{~g} \mathrm{~cm}^{-3}$, the effective density of a black hole.

Cygnus $\mathrm{X}-1$ is a close binary system where the compact object is believed to be a black hole. Discovered by Bowyer et. al. ${ }^{[1]}$ in 1965, Cyg X-1 is one of the brightest X-ray sources in the sky and one of the best studied X-ray sources. The compact object has ${ }^{[2]}$ a mass greater than $7 \mathrm{M}_{\odot}$ and a best estimate of $16 \pm 5 \mathrm{M}_{\odot}$ with a 5.6 day orbital period. The companion star is a supergiant (O9.7 Iab) with a mass greater than $20 \mathrm{M}_{\odot}$ and a best estimate of $33 \pm 9 \mathrm{M}_{\odot}$.

The binary system is additionally characterized by frequent transitions it undergoes between two states ${ }^{[3]}$ : a "low" state, where it spends about $90 \%$ of its time, and a "high" state. The two states are distinguished by their energy spectra shown in Figure 1.2 and their total luminosity. During the low state, the source emits primarily a hard Xray component, described by a single power-law spectrum over the range $1-250 \mathrm{keV}$ :

$$
d F / d E=b E^{-\alpha}
$$

where $d F / d E$ is the differential flux [photons $\mathrm{cm}^{-2} \mathrm{~s}^{-1} \mathrm{keV}^{-1}$ ], $E$ is the energy, $b$ is the proportionality constant and $\alpha$ is a constant called the spectral index. For Cyg X-1 in the low state, $\alpha$ ranges from $1.3-2.3$ and the total $X$-ray luminosity ${ }^{[4]}$ is about $3 \times 10^{37} \mathrm{erg} \mathrm{s}^{-1}$.

The rarer high state makes its transition over periods of days and lasts for about a month. An intense, ultrasoft spectral component appears in the 3-6 keV band, followed 


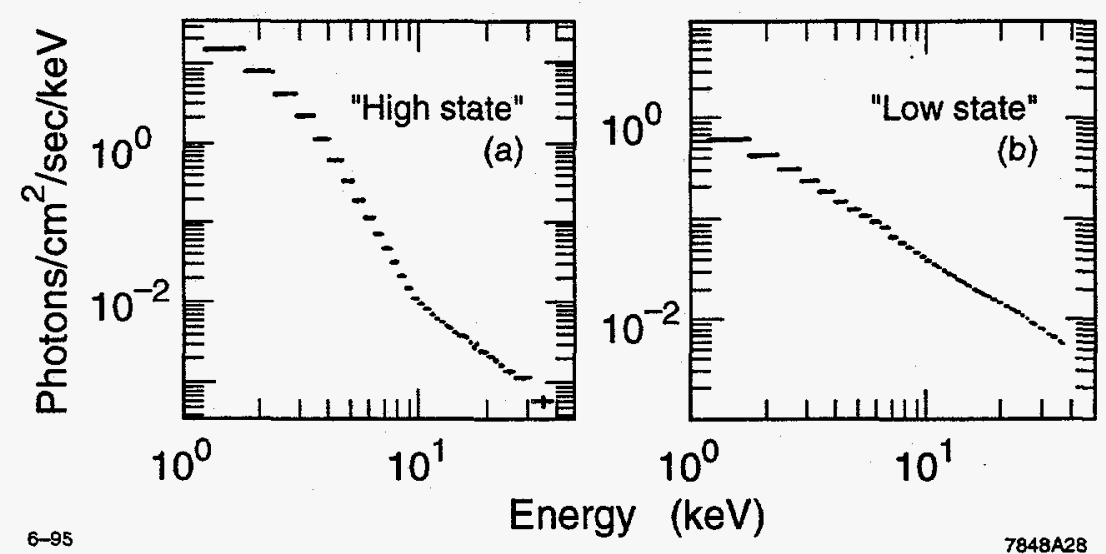

Fig. 1.2 X-ray energy spectra of Cyg X-1 in the a) high state and the b) low state. The low state is characterized by a single power law while the high state is described by a ultrasoft component plus a weaker hard power law tail.

by a weaker power-law above $10 \mathrm{keV}$ with $\alpha \sim 1.6-2.3$. The total luminosity in the high state increases by a factor of about 2 to over $6 \times 10^{37} \mathrm{erg} \mathrm{s}^{-1}$.

The Cyg X-1 data analyzed in this Thesis were collected from two instruments, the High Energy Astrophysical Observatory (HEAO) A-1 and the Rossi X-ray Timing Explorer (RXTE) Proportional Counter Array (PCA), described further in Section 1.3 and Chapters 3 and 4. During the HEAO A-1 observations, Cyg X-1 was in the low state ${ }^{[5]}$, with a mean energy flux of $1.25 \times 10^{-8} \mathrm{ergs} \mathrm{cm}^{-2} \mathrm{~s}^{-1}$ from $1-30 \mathrm{keV}$ assuming a power law with a spectral index of 1.56. An estimated distance to Cyg X-1 of $2.5 \mathrm{kpc}^{[6]}$ yields a mean source luminosity of $9.4 \times 10^{36} \mathrm{ergs} \mathrm{s}^{-1}$. The RXTE observation occurred while Cyg X-1 was in the high state ${ }^{[7]}$. The energy spectrum was fit to a $0.34 \mathrm{keV}$ soft blackbody component plus a power law between 3-200 keV with a spectral index of 2.5 . During the time of the RXTE observation, the bolometric luminosity was estimated to be $\sim 4.7 \times 10^{37}$ ergs s $^{-1}$ from observations of Cyg X-1 ${ }^{[7]}$ using the All-Sky Monitor/RXTE and BATSE/Compton Gamma Ray Observatory.

A black hole, as the name suggests, is a region of spacetime in which nothing, not even light, can escape. Predicted by general relativity, a black hole arises when the 
gravitational potential of matter becomes so high that nothing can stop self-gravitation from collapsing the matter into a singularity.

The mass distribution of a black hole distorts its surrounding vacuum spacetime geometry. For a non-rotating black hole in a vacuum, the spacetime geometry is described by the Schwarzschild metric ${ }^{[8]}$

$$
d s^{2}=-(1-2 M / r) d t^{2}+(1-2 M / r)^{-1} d r^{2}+r^{2} d \Omega^{2}
$$

where $M$ is the black hole mass. The quantity $r$ is the radius of a two-dimensional spherical surface whose proper circumference is $2 \pi r$. Unless otherwise specified, the units for this Chapter are chosen such that $c$ (speed of light $)=G$ (gravitational constant $)=1$. Schwarzschild derived this metric by solving the Einstein field equations in a vacuum assuming spherical symmetry. The surface of the black hole or event horizon lies at $R_{s}=$ $2 M \cong 3\left(M / M_{\odot}\right)[\mathrm{km}]$, commonly called the Schwarzschild radius.

For a test particle of mass $m$ and angular momentum $L$, the effective potential in the spacetime geometry defined by the Schwarzschild metric is ${ }^{[9]}$

$$
V(r)=m\left[(1-2 M / r)\left(1+L^{2} /(m r)^{2}\right)\right]^{1 / 2}
$$

such that the equation of motion for the test particle is given by

$$
m^{2}(d r / d \tau)^{2}=E^{2}-V(r)^{2}
$$

where $E$ is the energy of the particle and $\tau$ is the proper time of a comoving observer.

The effective potential is shown in Figure 1.3 for several values of the test particle's angular momentum. The local minima are represented by dots, corresponding to radii of stable circular orbits. Such orbits exist only for $L>2 \sqrt{3} M$. The innermost or marginally stable circular orbit occurs for a test particle with angular momentum $L=2 \sqrt{3} M$ at $r_{m s}=3 R_{s}=9\left(M / M_{\odot}\right)[\mathrm{km}]$, corresponding to the point of inflection of the effective potentials. 


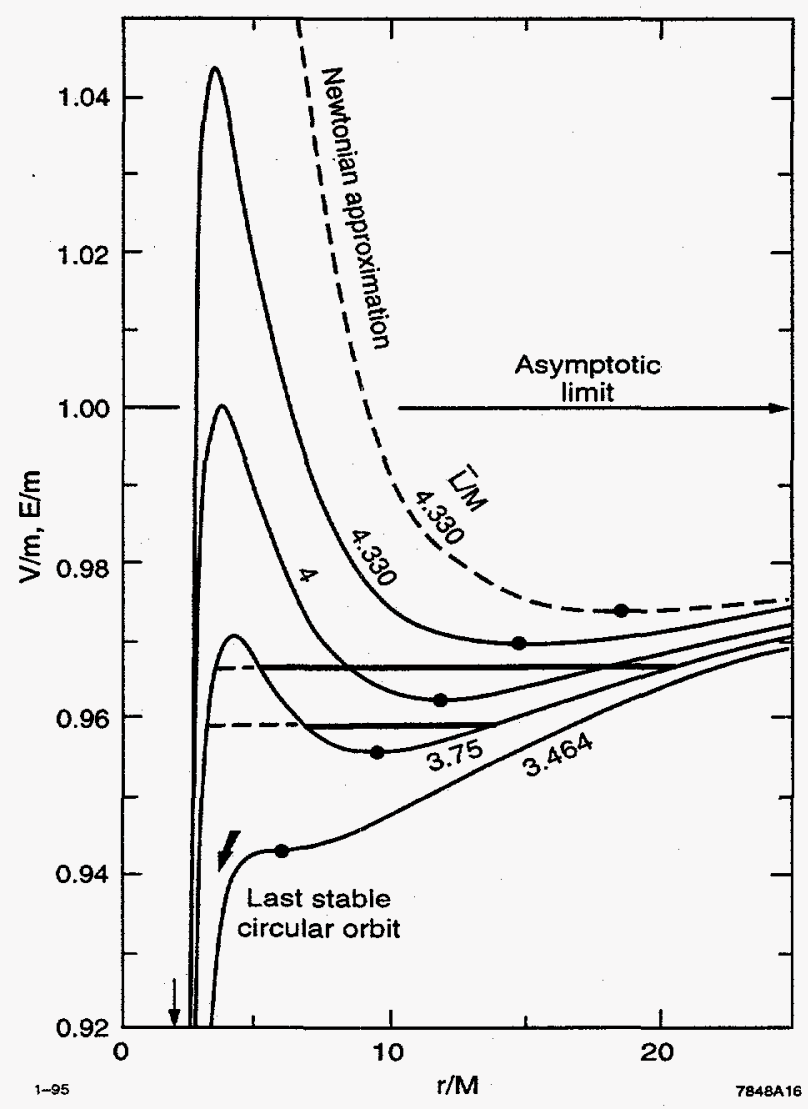

Fig. 1.3 The effective potential for a test particle of mass $m$ of various angular momenta $L$, orbiting a Schwarzschild black hole of mass $M$. The horizontal lines mark various energies of the test particle. The dots at the local minima of the effective potential curves identify radii of stable circular orbits. For comparison, the effective Newtonian potential is shown by the dashed line for one of the angular momenta.

When rotation of the black hole is considered, the radius of the marginally stable circular orbit becomes dependent on the black hole's angular momentum. For a chargeless rotating black hole, the spacetime geometry is described by the Kerr metric ${ }^{[10]}$. In Boyer-Linquist coordinates ${ }^{[11]}$, the Kerr metric is given by

$$
\begin{aligned}
d s^{2}= & -(1-2 M r / \Sigma) d t^{2}-\left(4 a r M^{2} \sin ^{2} \theta / \Sigma\right) d t d \phi+(\Sigma / \Delta) d r^{2} \\
& +\Sigma d \theta^{2}+\left(\dot{r}^{2}+a^{2} M^{2}+2 a^{2} r M^{2} \sin ^{2} \theta / \Sigma\right) \sin ^{2} \theta d \phi^{2}
\end{aligned}
$$

where $a \equiv c J /\left(G M^{2}\right), \Delta \equiv r^{2}-2 M r+a^{2} M^{2}$ and $\Sigma \equiv r^{2}+a^{2} M^{2} \cos ^{2} \theta$. The dimensionless angular momentum parameter, $a$ is defined here explicitly in terms of $c$ and $G$ for clarity. 
Setting $a=0$ in Equation [1.6] gives the Schwarzschild metric. Solved by Bardeen et. al. $^{[12]}$, the radius of the marginally stable circular orbit for the Kerr metric is given by

$$
\begin{aligned}
& r_{m s}=M\left(3+Z_{2} \mp\left[\left(3-Z_{1}\right)\left(3+Z_{1}+2 Z_{2}\right)\right]^{1 / 2}\right) \\
& Z_{1} \equiv 1+\left(1-a^{2}\right)^{1 / 3}\left[\left(1+a^{2}\right)^{1 / 3}+\left(1-a^{2}\right)^{1 / 3}\right] \\
& Z_{2} \equiv\left(3 a^{2}+Z_{1}^{2}\right)^{1 / 2}
\end{aligned}
$$

The upper sign refers to corotating or direct orbits where the orbiting test particle and black hole angular momenta are parallel, the lower sign to counterrotating or retrograde orbits. For non-rotating black holes $(a=0)$, Equation [1.7] reduces to $r_{m s}=6 M$. At maximum rotation $(a=1), r_{m s}=M=1.5\left(M / M_{\odot}\right) \mathrm{km}$ for direct orbits and $r_{m s}=9 M=$ $13.5\left(M / M_{\odot}\right) \mathrm{km}$ for retrograde orbits.

The radius of the marginally stable orbit defines the inner edge of the accretion disk around a black hole. The amount of gravitational binding energy at the inner edge of the disk is the total energy that is radiated by matter as it drifts inward through the disk. For a non-rotating black hole binary, the radiation efficiency for matter accreting onto the surface of the disk is $5.7 \%$, increasing to up to $42 \%$ for a maximally rotating black hole. For a white dwarf or a neutron star binary, the inner edge of the accretion disk may extend all the way down to the compact object's surface, leading to radiation efficiencies of $0.01 \%$ and $10 \%$, respectively. Comparing these efficiencies to that of nuclear burning, $0.9 \%$, the conversion of rest mass to other forms of energy for accretion onto disks around black holes and neutron stars is considered highly efficient.

Most of the radiation from accreting matter originates from the innermost regions of the accretion disk. A thin, Keplerian accretion disk around a black hole has an integrated flux emitted from the top and bottom faces of the disk of ${ }^{[13]}$

$$
F(r) \approx \frac{3}{4 \pi} \frac{L}{r^{2}}\left(\frac{r_{m s}}{r}\right)\left[1-\left(\frac{r_{m s}}{r}\right)^{1 / 2}\right]
$$




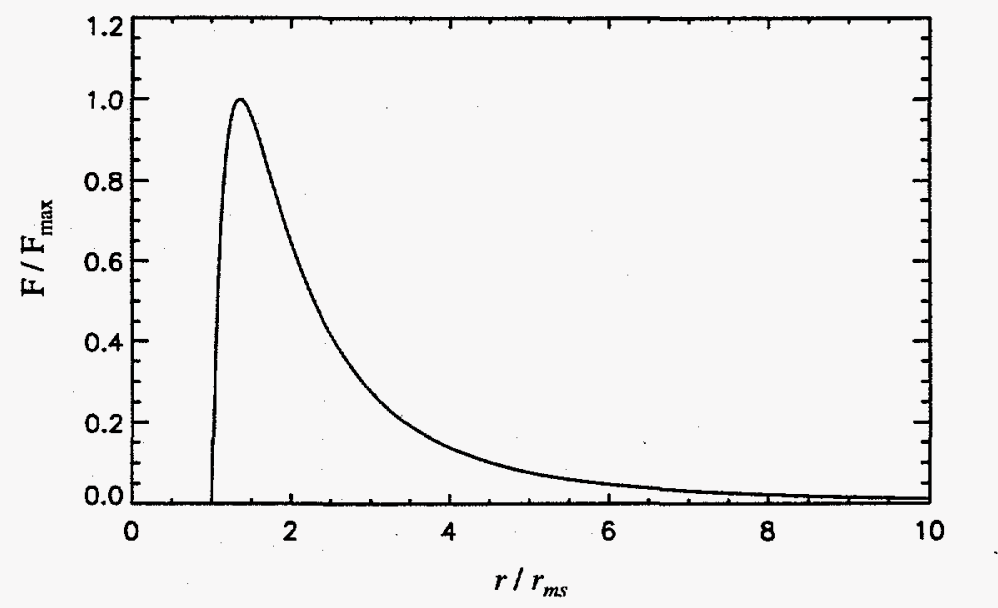

Fig. 1.4 The radial distribution of the integrated flux emitted from the faces of a thin, Keplerian accretion disk. Most of the radiation originates from the innermost parts of the disk. The peak of the distribution occurs at $1.4 r_{m s}$, where $r_{m s}$ is the radius corresponding to the innermost stable orbit.

where $L$ is the total luminosity of the disk. As Figure 1.4 shows, the integrated flux distribution is strongly peaked at $1.4 r_{m s}$. Accreting matter is drawn to the inner disk regions due to interactions with viscous stresses in the disk that transport angular momentum outward from the inner to the outer regions of the disk. The narrow peak of flux distribution observed in Figure 1.4 reflects the viscous stress that is seen by the accreting matter as it falls towards the compact object.

The standard thin disk models obtained by Shakura and Sunyaev ${ }^{[14]}$ and by Novikov and Thorne ${ }^{[15]}$ successfully characterize some of the energy spectra of radiation seen from binary systems. If the disk is optically thick, the expected emitted radiation is a modified black body spectrum described by the differential flux $F_{v}$,

$$
F_{v} \propto \frac{x^{3 / 2} \exp (-x / 2)}{\left(e^{x}-1\right)^{1 / 2}}
$$

where $x \equiv h v / k T_{s}$ and $T_{s} \approx 10^{9} \mathrm{~K}$ is the characteristic temperature of the accretion disk surface at which the emergent photon spectrum is formed. Equation [1.9] successfully describes the ultrasoft spectrum observed in the high state of Cyg X-1. 
However, the standard thin disk models cannot produce the hard X-rays $(\sim 100$ $\mathrm{keV}$ ) observed from Cyg X-1 ${ }^{[15]}$. Other disk models have been proposed to explain the hard X-rays by adding Comptonization mechanisms to the existing standard thin disk models. Thorne and Price ${ }^{[16]}$ have argued that the instability in the inner region of a disk could swell the usual optically thick, radiation-pressure dominated region to a hotter $\left(\sim 10^{9} \mathrm{~K}\right)$, gas-pressure dominated, optically thin region. This would result in a "Compton cloud" occupying the inner region of a disk. By Compton scattering soft X-ray photons into hard X-rays, the Compton cloud model predicts a power-law for the spectral curve from 8 to $\sim 500 \mathrm{keV}$, which compares well with the observed hard X-ray spectrum from Cyg X-1.

Another disk model first proposed by Ostriker ${ }^{[17]}$, Liang and Price ${ }^{[18]}$, and Bisnovatyi-Kogan and Blinnikov ${ }^{[19]}$, consists of an optically thick disk surrounded by a hot corona. The high temperatures in the corona are produced by acoustic and Alfvén waves and magnetic dissipation. The hot, thermal electrons in the corona Comptonize the soft photons into hard X-rays.

Recent advective-dominated accretion disk models proposed by Chakrabarti and Titarchuck $^{[20]}$ and Narayan ${ }^{[21]}$ provide a self-consistent explanation for both the ultrasoft and hard power law tail energy spectra of Cyg X-1. The Two-Component Advective Flow (TCAF) model ${ }^{[20]}$ consists of an optically thin sub-Keplerian halo sandwiching an optically thick Keplerian disk, as illustrated in Figure 1.5. The disk component consists of viscous, predominantly Keplerian gases accreted through the Lagrange point from the companion star. As in the standard thin disk models of Shakura and Sunyaev, soft X-ray photons are expected to dominate the distribution of radiation emitted from the Keplerian disk. The surrounding halo component consists of a low viscosity, sub-Keplerian gas that is fed from the Keplerian disk far away and contributed to by companion winds.

The low angular momentum, sub-Keplerian gas flows advectively towards the black hole with roughly constant angular momentum, building up ram pressure due to its radial motion as it nears the centrifugal barrier. Where the ram pressure of the pre-shock flow roughly matches with the thermal pressure of the rotation dominated post-shock 


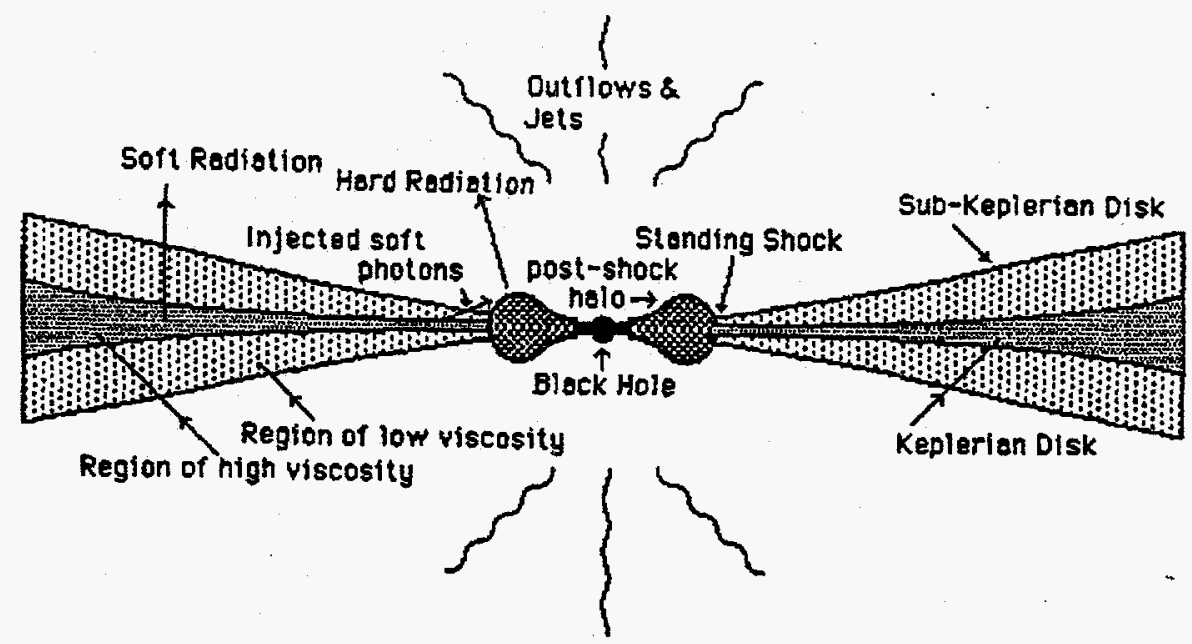

Fig. 1.5 Schematic diagram of the two component advective disk model. A Keplerian disk component is enveloped by a sub-Keplerian halo component. A standing shock forms near where the sub-Keplerian gas hits the centrifugal barrier. Jets emitted from the post-shock region are predicted for supermassive black holes $\left(M>10^{7} \mathrm{M}_{\odot}\right)$.

flow a standing shock wave is formed. For matter with marginally bound angular momentum, $2 \sqrt{3} M$, the shock forms around $\sim 10-30 R_{s}$. The location of the standing shock defines the effective inner edge of the accretion disk for both the disk and halo components. The post-shock thermalizes the accretion kinetic energy from the subKeplerian halo and a creates hot, optically slim and geometrically thick post-shock region. The post-shock halo intercepts soft photons from the Keplerian component and reradiates them as hard $\mathrm{X}$-rays after Comptonization.

Figure 1.6 shows how the TCAF model describes the spectral index of the low and high states of black hole binary systems as a function of the accretion rate of the disk $\partial_{t} m_{d}=\partial_{t} M_{d} / \partial_{t} M_{E d d}$ and the accretion rate of the halo $\partial_{t} m_{h}=\partial_{t} M_{h} / \partial_{t} M_{E d d}$. The Eddington accretion rate $\partial_{t} M_{E d d}$ is proportional to the Eddington luminosity ${ }^{[22]}$,

$$
L_{E d d}=\frac{4 \pi G M m_{p} c}{\sigma_{T}}=1.3 \times 10^{38}\left(M / M_{\odot}\right) \mathrm{erg} \mathrm{s}^{-1}
$$

which is derived by balancing the gravitational attraction from the compact object's mass to the the radiation pressure from the photons emitted from the disk. For a $16 \mathrm{M}_{\odot}$ black hole in Cyg X-1, the Eddington limit is $2 \times 10^{39} \mathrm{erg} \mathrm{s}^{-1}$. 


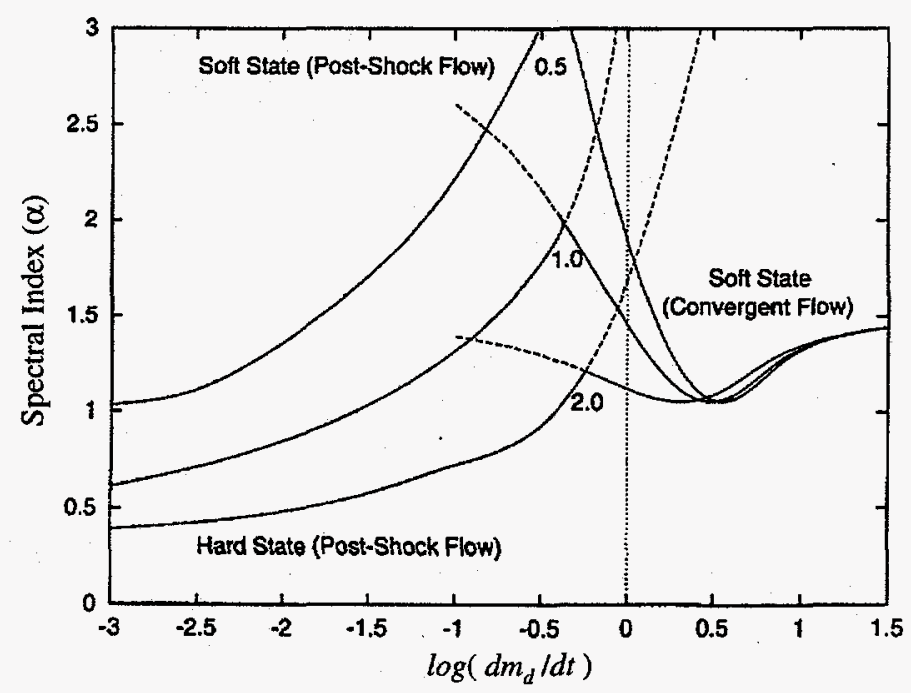

Fig. 1.6 Variation of the energy spectral index $\alpha$ (observed slope in the 2-50 $\mathrm{keV}$ region) for the TCAF model ${ }^{[20]}$ as functions of the disk and halo accretion rates. Halo rates are marked on the curve. Computed spectral index in soft states due to convergent pre-shock inflow from the sub-Keplerian halo is also provided. Dashed curves indicate regions where both components could contribute. $M=5 M_{\odot}$ is chosen.

During the low state the soft photons emitted from near the inner edge of the Keplerian disk (i.e. at the standing shock) are reradiated as hard X-rays by the post-shock halo through Comptonization. This accounts for the observed hard power-law energy spectrum. During the high state, when the accretion rate is higher, the additional flux of soft photons cools the post-shock region through inverse Compton scattering. This leads to a suppression of the hard power-law tail and an enhancement of the ultrasoft spectrum.

All of the accretion disk models described are steady state models that provide little temporal information about the X-ray emissions from the accretion process. However, there are several models that attempt to describe the time variability of X-ray emissions from a black hole binary.

Bao and $\varnothing_{\text {stgaard }}{ }^{[23]}$ have numerically modelled orbiting blobs in a geometrically thin accretion disk around a black hole including all relativistic effects. The blobs or "hot spots" were simulated to radiate photons isotropically in their proper rest frames. For various assumed blob distributions and different inclination angles, they find that the time variability power spectra exhibit drop-offs at the Keplerian frequency of the 


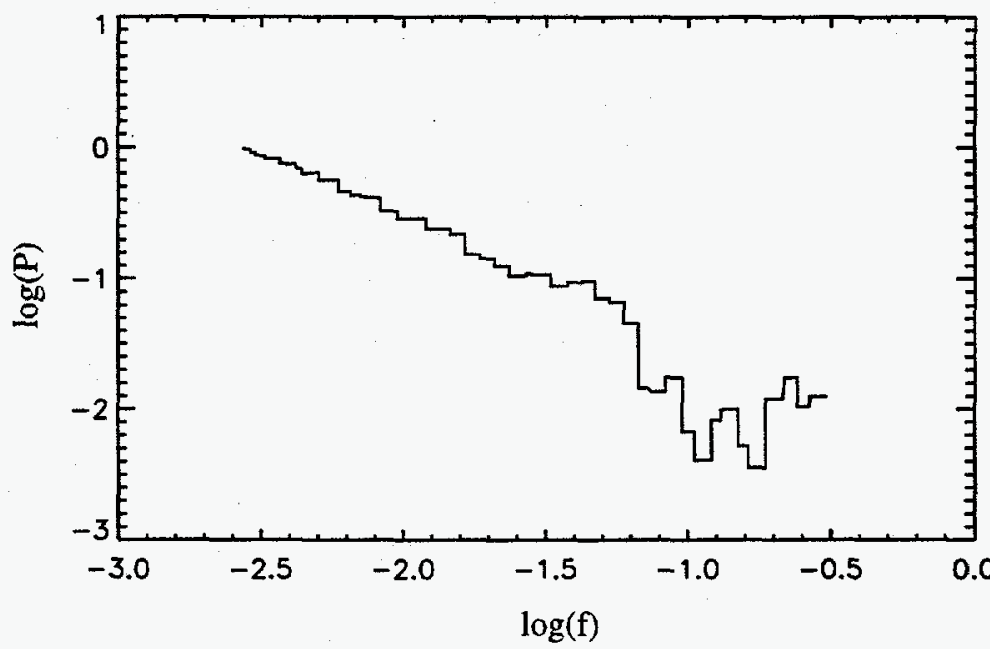

Fig. 1.7 Power spectrum of many simulated optically thick blobs orbiting about a black hole. Units for power are arbitrary and units of frequency are in $-1.5 \log (r / M)$. The drop-off in power occurs at the innermost orbit of the blobs corresponding to the Keplerian frequency $-1.5 \log (6)=-1.2$. The inclination of the accretion disk is $40^{\circ}$ and the number distribution for the blobs, $n(r) \sim r^{-0.5}$.

inner edge of the accretion disk. These drop-offs are present whether the disk is optically thick or optically thin. Figure 1.7 shows an example of one of their power spectra that exhibits such a drop-off. The drop-off signifies an end of the self-similar $1 / f$ structure of the variability. While Figure 1.7 shows a sharp drop-off in the power spectra, Bao and Østgaard recognized that noise in the observation data may smooth out this feature.

A model of accretion disk turbulence by Nowak and Wagoner ${ }^{[24]}$ also predicts a sharp drop-off in the Fourier power spectrum as shown in Figure 1.8. Three-dimensional hydrodynamic turbulence was considered as the mechanism driving accretion disk viscosity, affecting the observed flux by coupling to acoustic modes. In addition, lowfrequency variability was modelled by weak fluctuations on viscous/thermal time-scales. For a $6 \mathrm{M}_{\odot}$ black holeand a detector bandpass from 1.2 to $15.7 \mathrm{keV}$, their model predicts a sharp rollover in the Fourier power spectrum that starts between $\sim 20-60 \mathrm{~Hz}$ and falls as $f^{-5}$ for frequencies $f \geq 100 \mathrm{~Hz}$. 


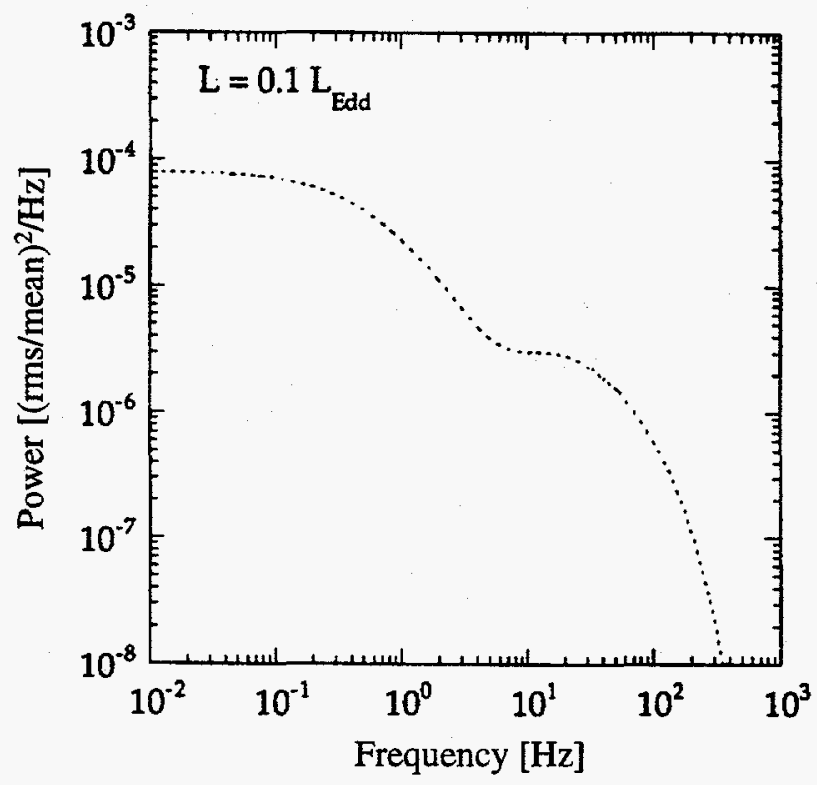

Fig. 1.8 Combination of viscous/thermal timescale and acoustic mode power from a model of accretion disk turbulence by Nowak and Wagoner ${ }^{[24]}$. The detector bandpass $=1.2-15.7 \mathrm{keV}$; the luminosity $=0.1$ times the Eddington limit; the viscosity parameter $\alpha=0.3$; and the mass of the black hole $=6 \mathrm{M}_{\odot}$.

In contrast to drop-offs in variability, Kato and Fukue ${ }^{[25]}$ realized that normal modes of oscillations can be trapped by general relativity near the inner edge of accretion disks around black holes. These modes do not exist in Newtonian gravity. Recent progress has been made in quantitatively identifying these modes. Nowak and Wagoner ${ }^{[26]}$ used a modified Newtonian potential to identify and characterize two of these modes. Perez, et. al. ${ }^{[27]}$ reanalyzed these modes by applying the relativistic fluid perturbation formalism of Ipser and Lindblom ${ }^{[28]}$ to thin accretion disks about a Kerr black hole. These studies of adiabatic oscillations that are trapped in the inner regions of accretion disks by non-Newtonian gravitational effects of a black hole have been called relativistic diskoseismology ${ }^{[27]}$.

Three types of trapped adiabatic oscillations have been identified and characterized by Nowak and Wagoner and Perez, et. al. ${ }^{[26],[27]}$ : internal gravity $(\mathrm{g})$ modes, acoustic (p) modes and corrugation (c) modes. The lowest g-mode oscillations result from vertical displacements of the disk. Small radial displacements trapped near the inner 
radius characterize the lowest $\mathrm{p}$-mode. The c-mode oscillations are nonradial incompressible waves that slowly precess about the angular momentum of the black hole.

These trapped oscillations are expected to manifest themselves in the observed Xray fluctuations as quasi-periodic oscillations (QPOs). The quality factor, $Q$ of these QPOs can range from $\sim 1$ to $\sim 100$ depending on the disk viscosity and the number of radial and vertical modes excited. Nowak and Wagoner ${ }^{[26]}$ obtained an estimation of the fractional modulation of the disk luminosity to be $\sim 1 \%$.

A prominent observed characteristic in black hole binaries such as Cyg X-1 is the power law shape of its variability power spectra (PSD). Attempts have been made to explain this power law using phenomenological models. The shot-noise model ${ }^{[29]}$ in particular, has been very successful in reproducing power law spectra. Each shot consists of an exponential rise and decay and the shots are distributed randomly in time. Variants of the shot model have also been proposed, such as the self-organized criticality (SOC) model proposed by Bak et al. ${ }^{[30]}$ and later applied to black hole objects by Mineshige, Ouchi and Nishimori ${ }^{[31]}$. Here it is assumed that the regions near the inner edge of the disk are populated by numerous small reservoirs. If a critical mass density is reached at some reservoir, an unknown instability becomes established and accumulated material drifts inward as an avalanche, thereby emitting $X$-rays. The size of the X-ray shot is proportional to the number of reservoirs involved in an avalanche.

\subsection{Searching for Black Hole Signatures in Cygnus X-1}

Cygnus X-1 has been studied extensively for over 30 years because of several observed characteristics that indicate that its compact object is a black hole. Finding these characteristics or signatures is important in determining if black holes really exist. Signatures may also help determine one or more of the three physical properties of the black hole: its mass, angular momentum and charge. If black hole and neutron star binaries can be distinguished from each other, then observational characteristics unique to either a black hole or a neutron star may be identified. Finally, searching for expected 
black hole signatures tests models of the accretion process and its underlying physics, general relativity.

Currently, the most popular and easiest method for identifying a compact object as a potential black hole is if its mass exceeds the Rhoades \& Ruffini limit ${ }^{[32]}$ of $3.2 \mathrm{M}_{\odot}$. The mass limit results from integrating over the volume of a gravitationally bound compact object in general relativistic hydrostatic equilibrium assuming an equation of state with the greatest possible stiffness. Matter cannot be so stiff that the speed of sound exceeds the speed of light, and this sets an upper limit on how strongly repulsive nuclear forces can become at ultrahigh densities. The Baym-Pethick-Sutherland equation of state $^{[33]}$ is assumed below nuclear densities, but the resulting mass contribution amounts to only a few percent of the total mass limit. However, the Rhoades \& Ruffini limit assumes that the compact object is bound gravitationally and that the nuclear physics of normal matter can be extrapolated to compact objects where the number of nucleons can exceed $10^{57}$. Other equations of states have been proposed ${ }^{[34]}$ that circumvent these two assumptions, leading to much larger mass limits for compact objects.

Additional evidence for the existence of black holes in close binary systems has been sought by studying the characteristics of the X-ray emissions. Initial studies of the $\mathrm{X}$-ray spectral data showed much promise for such evidence. Observed blackbody and power law energy spectra may be understood from standard ${ }^{[14],[15]}$ or advectiondominated ${ }^{[20],[21]}$ accretion disk models. However, these spectral characteristics appear to be mostly independent of the nature of the compact object since they are observed in both black hole and neutron star binary systems ${ }^{[35]}$.

The challenge remains to find $\mathrm{X}$-ray characteristics that are unique to black hole binary systems. One of the physical properties that distinguishes a black hole from a neutron star is the absence of a solid surface. A non-rotating black hole's "surface" is characterized by an event horizon at the Schwarzschild radius, $R_{s}$. Nothing within this surface can be emitted to the outside observer. Nothing from the outside can reflect off this surface. These properties are in stark contrast to those of a neutron star. In close binary systems, X-ray bursts can be emitted from a neutron star's surface due to 
thermonuclear flashes ${ }^{[36]}$. Isolated neutron stars called pulsars have intense magnetic fields that can beam $X$-rays out along their magnetic poles ${ }^{[37]}$.

The dynamic timescale associated with a black hole is defined by the proper circumference at its surface, $2 \pi R_{s}$. Light emitted from this spatial region should have a dynamic timescale $t_{d} \approx 2 \pi R_{s} / c$. For a $16 M_{\odot}$ black hole, the dynamic timescale is roughly one millisecond. Since $t_{d}$ corresponds to light emitted from the smallest spatial region around a black hole, no variability is expected at timescales below this characteristic value. In terms of frequency, this dynamic timescale becomes

$$
f_{d}=c\left(2 \pi R_{s_{\odot}}\right)^{-1}\left(M_{\odot} / M\right)=16\left(M_{\odot} / M\right) \mathrm{kHz}
$$

where $R_{s_{\odot}}$ is the Schwarzschild radius of a black hole with a mass of the sun. For Cyg X1 , the range of possible black hole masses, 7 to $21 M_{\odot}$, correspond to dynamic frequencies $2.3 \mathrm{kHz}$ to $758 \mathrm{~Hz}$, respectively. At the most probable mass, $16 M_{\odot}$, the dynamic frequency is $<1 \mathrm{kHz}$.

The dynamic frequency sets a rough upper limit to measuring variability in excess of Poisson noise. However, for close binary systems variability is expected to drop-off near the Keplerian frequency of the marginally stable circular orbit, as shown by Bao and $\emptyset_{\text {stgaard }}{ }^{[19]}$. The motion of a test particle about a central mass is characterized by its orbital frequency. The Keplerian frequency refers specifically to circular equatorial orbits. It is determined by finding the local minimum of the effective potential for radial motion introduced by the spacetime metric. For a rotating black hole governed by the Kerr metric the Keplerian frequency is ${ }^{[13]}$

$$
f_{k}=31.8\left(M_{\odot} / M\right)\left(\left(2 r / R_{s}\right)^{3 / 2} \pm a\right)^{-1} \mathrm{kHz}
$$

where the upper and lower signs refer to direct and retrograde orbits, respectively. In the limit of a non-rotating black hole $(a=0)$. with a mass of $16 M_{\odot}$, a test particle at the marginally stable orbit rotates with a Keplerian frequency of $135 \mathrm{~Hz}$.

By substituting the radius of marginally stable circular orbit for the Kerr metric, equation [1.7] into equation [1.14], the Keplerian frequency can be expressed as a 


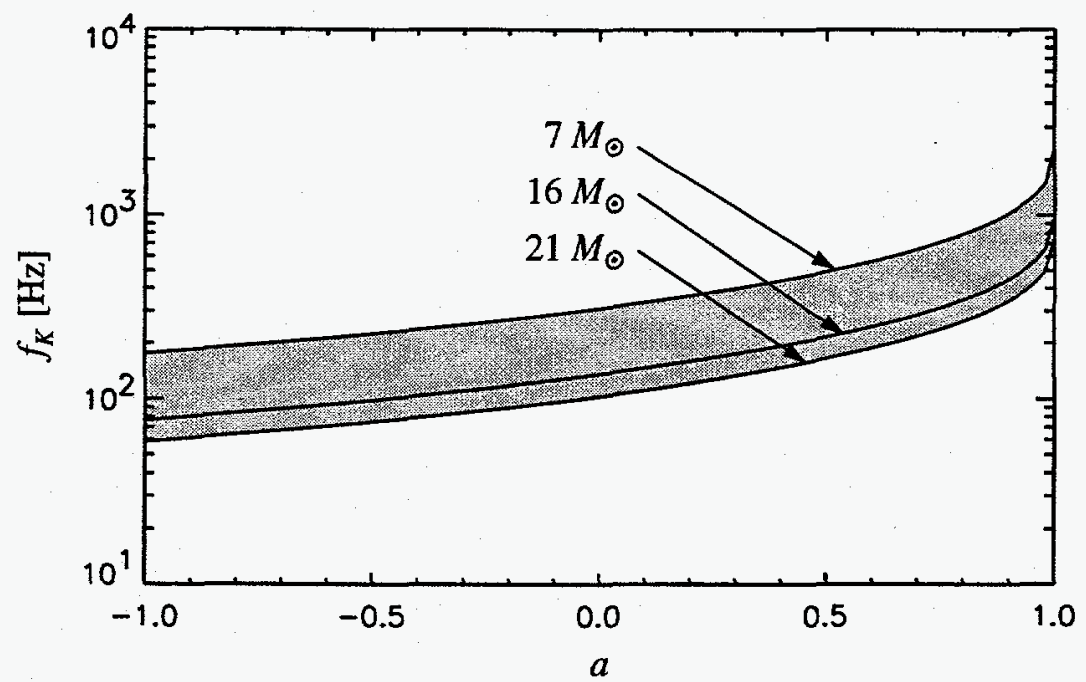

Fig. 1.9 The dependence of the Keplerian frequency at the marginally stable circular orbit on the black hole angular momentum parameter $a=$ $c J / G M^{2}$. Positive and negative values of $a$ represent direct and retrograde orbits, respectively. The boundaries of each band are defined by the mass limits of Cyg X-1's black hole.

function of the black hole's mass $M$ and angular momentum parameter $a$. Figure 1.9 shows the dependence of the Keplerian frequency over the ranges of possible $a$ and black hole masses of Cyg X-1. Positive and negative values of $a$ correspond to direct and retrograde orbits, respectively. The shaded band in Figure 1.9 covers frequencies from $58 \mathrm{~Hz}$ to $2.3 \mathrm{kHz}$.

For the advection-dominated TCAF disk model, the effective inner edge of the disk lies farther out from the marginally stable orbit at the edge of an expected standing shock. The location of the standing shock is a function of the black hole's angular momentum and several properties of the accretion disk. Viscosity, the efficiency for transferring heat, and the specific energy and angular momentum of the accreting gas all contribute to determining the location of the shock. These properties are insensitive to the accretion rate ${ }^{[20]}$, so the location of the standing shock is expected to be remain unchanged whether the binary system is in the high or low state.

For accreting gas with marginally bound angular momentum, the location of the standing shock ranges from $\sim 10-30 R_{s}$ to $\sim 5 R_{s}$ for a non-rotating $(a=0)$ and maximally 
rotating $(a=0.99)$ Kerr black hole, respectively ${ }^{[20]}$. At these radii, the Keplerian frequencies for the range of black hole masses, $7-21 M_{\odot}$ are from $51-3.3 \mathrm{~Hz}$ for $a=0$, and from $139-46 \mathrm{~Hz}$ for $a=1$, respectively. For a non-rotating $16 M_{\odot}$ black hole the Keplerian frequency is $22 \mathrm{~Hz}$ if the shock lies at $\sim 10 R_{s}$.

Unlike the radius of a marginally stable circular orbit, the effective inner edge of an advection-dominated accretion disk may not be as sharply defined. Several shock characteristics such as its strength may affect how well the shock obscures the Keplerian disk component behind the centrifugal barrier ${ }^{[20]}$. A partial obstruction of this Keplerian disk component may smear this effective edge.

Other possible signatures of a black hole in a close binary system are the non-Newtonian quasi-periodic oscillations (QPOs) predicted by relativistic diskoseismology. ${ }^{[26],[27]}$. The QPOs result from modes of oscillations trapped near the inner edge of the accretion disk. Of the three types of identified trapped oscillations, the g-modes are the most robust and observable. The lowest radial $(m=0)$ g-modes have a frequency given by ${ }^{[27]}$

$$
f_{g}=714\left(1-\varepsilon_{n j}\right)\left(M_{\odot} / M\right) F(a) \mathrm{Hz}, \quad \varepsilon_{n j} \approx\left(\frac{n+1 / 2}{j+1}\right) \frac{h}{r_{0}}
$$

The dependency of $f_{g}$ on the properties of the accretion disk is limited to one small term, $\varepsilon_{n j}$, in which $r_{0}$ is the radius of the mode, $2 h\left(r_{0}\right)$ is the disk thickness, and $n$ and $j$ are the radial and vertical mode numbers, respectively. A radiation-pressure dominated optically thick disk region has typical geometrical fractional thickness $h\left(r_{0}\right) / r_{0} \sim 0.1 L / L_{E d d} \sim 10^{-3}$ for Cyg X-1. $F(a)$ depends on the black hole angular momentum parameter $a=$ $c J /\left(G M^{2}\right)$, increasing from $F(-0.99)=0.60$ to $F(0.99)=3.44$. For a non-rotating, $16 M_{\odot}$ black hole, Equation [1.15] gives $\sim 45 \mathrm{~Hz}$ for the lowest g-mode frequency.

Using [1.15], the frequencies of the g-modes of oscillations are plotted in Figure 1.10 for the same three black hole masses chosen for the Keplerian frequency profile. The g-mode frequencies range from $20.4 \mathrm{~Hz}$ at $a=-1$ to $351 \mathrm{~Hz}$ at $a=1$. The frequency 


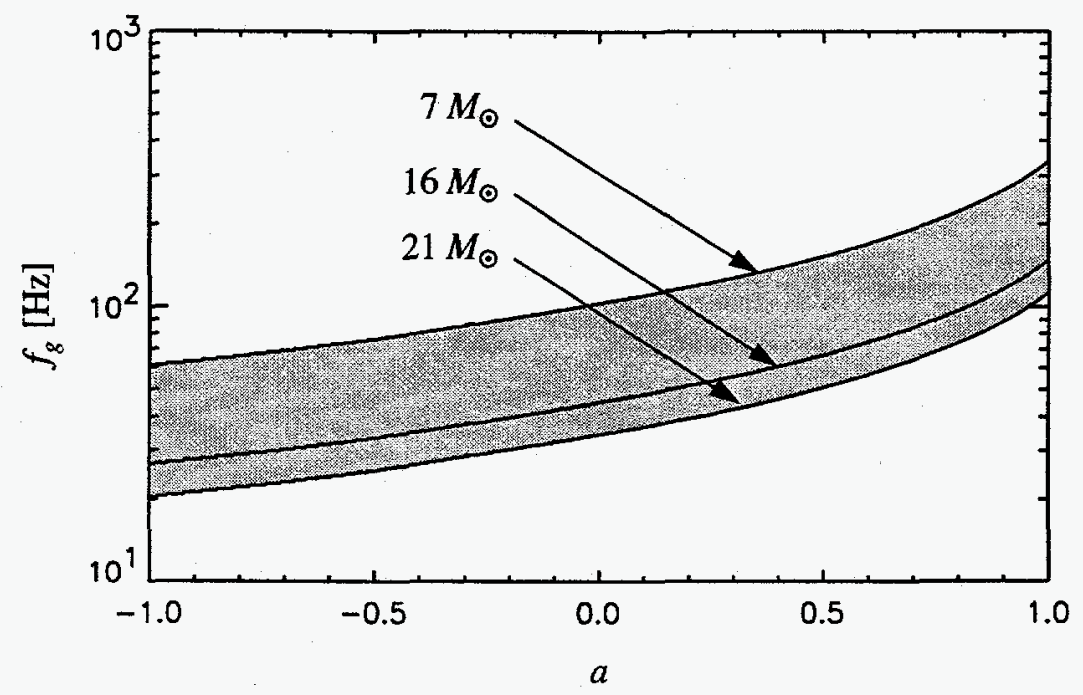

Fig. 1.10 The dependence of the frequency of the lowest radial ( $m=0)$ g-modes on the black hole angular momentum parameter $a=c J / G M^{2}$. Positive and negative values of $a$ represent direct and retrograde orbits, respectively. The boundaries of the band are defined by the mass limits of Cyg X-1's black hole.

band for these g-modes lies just underneath the frequency band for direct marginally stable orbits shown in Figure 1.9.

A summary of all the different characteristic frequencies predicted from these accretion disk models of Cyg X-1 are shown in Figure 1.11, spanning from roughly $3 \mathrm{~Hz}$ to $2.5 \mathrm{kHz}$. For each shaded region, the range of frequencies covers the ranges of possible black hole mass ( 7 to $21 M_{\odot}$ ) and angular momenta ( $a=0$ to 1 ).

To search for these variability characteristics, the Fourier spectral technique is employed. The finite Fourier transform of a time series of length $T$ divided into $N$ equal-length bins is

$$
a_{j}=\sum_{k=0}^{N-1} x_{k} e^{2 \pi i j k / N} \quad(j=0,1,2 \ldots N / 2)
$$

where $x_{k}$ is the number of events in the $k$ th time bin, and $a_{j}$ is the Fourier coefficient at frequency $f_{j}\left(f_{j}=j / T\right)$. The squared magnitude of the $j$ th Fourier coefficient, $\left|a_{j}\right|^{2}$, is a measure of the variability power in the source at frequency $f_{j}$. 


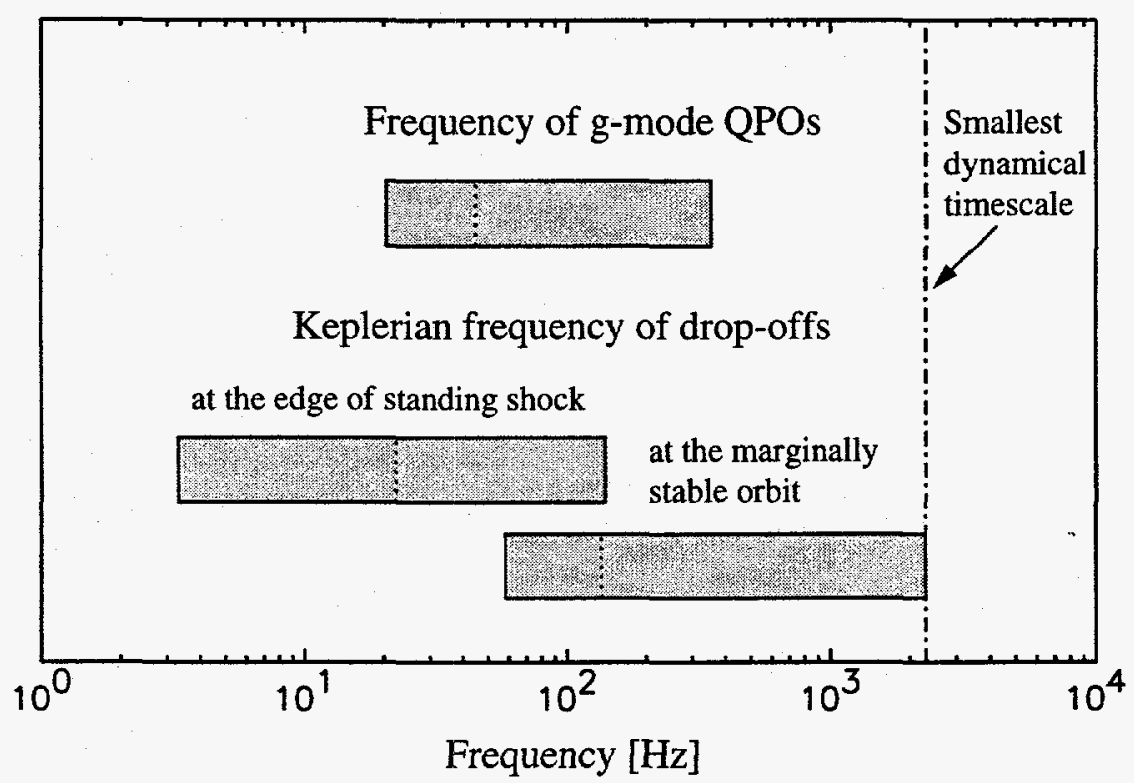

Fig. 1.11 Summary of the frequencies where drop-offs or QPOs are predicted in Cyg X-1 over the range of possible black hole mass and angular momentum. The dashed vertical line in each shaded region marks the predicted frequency for a non-rotating, $16 M_{\odot}$ black hole.

The distribution of the variability power over all possible frequencies forms the Fourier power spectrum. With the Leahy normalization, this power spectrum is given by $^{[38]}$

$$
P_{j}=\frac{2\left|a_{j}\right|^{2}}{N_{\gamma}}
$$

where $N_{\gamma}$ is the total number of photons observed in the interval from 0 to $T$. ( $N_{\gamma}$ equals the value of the zeroth Fourier coefficient, $a_{0}$ ).

For a steady source, the power given by [1.17] is not zero because of noise introduced by Poisson counting statistics. However, Poisson noise introduces the same amount of power at all frequencies, and the power introduced can be readily calculated from the count rate. Excluding any instrumental effects, the power in [1.17] due to Poisson noise is 2 at every frequency ${ }^{[38]}$, forming what is usually called the Poisson noise floor. 
At a given frequency, the powers introduced by Poisson noise are normally distributed by the central limit theorem. Namely, $P_{j}$ is distributed as $\chi^{2}$ with 2 degrees of freedom, where the mean is equal to the standard deviation. Fourier transforming a longer time series simply extends the frequency range where power is calculated. The power at each frequency is still distributed with the same standard deviation.

To improve the signal-to-noise, it is necessary to sum a large number of power spectra of independent segments of data. In addition, the powers are usually summed over adjacent frequency bins in a narrow interval. The actual procedure consists of first dividing the data into sequential time segments of equal length. The Fourier power spectra are then calculated for all of these segments and then averaged together over equal logarithmic frequency intervals. If the power spectrum is averaged over $S$ independent data segments and $M$ adjacent frequency bins then the distribution of averaged power due to Poisson noise approaches a Gaussian with standard deviation $2 / \sqrt{S M}$. An intrinsic average power spectrum may be determined by subtracting the Poisson noise floor from the average power spectrum. The standard error on the average noise-subtracted power at a given frequency is determined by adding in quadrature the standard errors of the average power spectrum and the Poisson noise floor.

\subsection{Experimental Results}

Results from analysis of Cyg X-1 data from HEAO A-1 and RXTE/PCA, detailed in Chapter 3 and 4, are presented here. HEAO A-1 and RXTE/PCA have time resolution capabilities of 10 microseconds or better. Such capabilities are useful to search for black hole characteristics predicted at the frequencies summarized in Figure 1.11. Studies of the HEAO A-1 data were motivated by evidence for the inner edge of an accretion disk found in one of its observations of Cyg X-1. An analysis of that observation by Meekins, et. al. ${ }^{[6]}$ showed evidence for excess intrinsic variability between the $1-10$ millisecond timescales that cut off at the one millisecond timescale. For a $16 M_{\odot}$ black hole, the marginally stable orbit lies at a timescale of $\sim 7$ milliseconds and the dynamic timescale of equation [1.13] is expected at one millisecond. 
Encouraged by this possible black hole signature, this evidence was first reproduced by applying the same analysis to the same observation. Figure 1.12a shows good agreement between the original and reproduced analyses. In their analysis of the HEAO A-1 data, Meekins, et. al. neglected the effects due to dead time and other possible instrumental effects. After an extensive analysis of all the high time resolution HEAO A-1 data and a comparison to Monte Carlo models of the detector response, a previously undetected instrumental effect was found. This instrumental effect has been observed in all the high time resolution pointed observations. A HEAO A-1 module-electronics malfunction is suspected of causing these distortions. Chapter 3 describes this instrumental effect in further detail. Figure $1.12 \mathrm{c}$ shows the result of correcting the Meekins, et. al. analysis for dead time and other instrumental effects using a Leahy normalized Fourier power approximation to the relative power described in Chapter 3. After correcting for instrumental effects, the excess intrinsic variability between the 1-10 millisecond timescale is no longer present.

The Fourier power spectral technique was then applied to all the high time resolution Cyg X-1 data from HEAO A-1. Figure 1.13 shows the resulting noisesubtracted Fourier power spectrum. The noise floor due to Poisson statistics was corrected for instrumental effects before the subtraction, described further in Chapter 3. To measure the significance of the noise-subtracted power, the 95\% confidence level for detecting a signal above the Poisson noise floor is overlayed in Figure 1.13 as a solid curve. Excess power is observed at frequencies less than $20 \mathrm{~Hz}$ at greater than a $95 \%$ confidence level above the Poisson noise. At frequencies greater than $40 \mathrm{~Hz}$, the noisesubtracted spectrum was forced to be consistent with the null hypothesis in order to correct the Poisson noise floor for HEAO A-1's instrumental effects.

Between frequencies 0.1 and $20 \mathrm{~Hz}$, the spectrum follows power laws with a break to a steeper index at $3 \mathrm{~Hz}$. Power laws were fit separately to the frequency region before and after the break. As Table 1.1 shows, the power spectrum roughly follows a $1 / f$ spectrum before the break and then falls more steeply afterwards. 

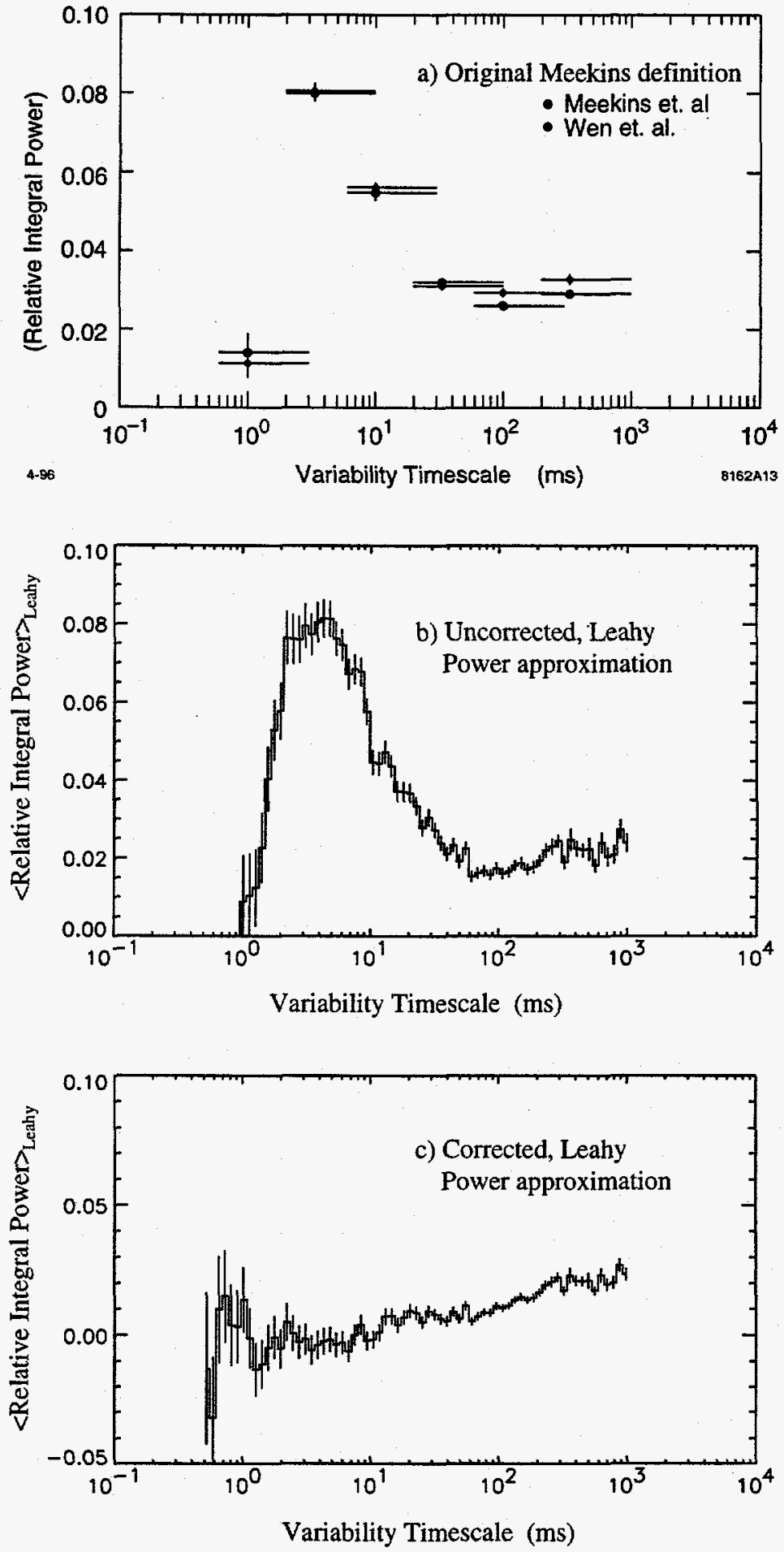

Fig. 1.12 The relative integral power defined by Meekins, et. al. for Cyg X-1, using a) the original Meekins definition, b) the Leahy power approximation, and c) the Leahy approximation corrected for dead time and additional instrumental effects. The two sets of relative integral power in a) are from the original Meekins, et. al. analysis and a reanalysis by Wen, et. al. using the general theoretical framework of Meekins, et. al. 


\begin{tabular}{|l|c|c|c|c|c|c|}
\cline { 2 - 7 } \multicolumn{1}{c|}{} & \multicolumn{2}{|c|}{ Power Law Before Apparent Break } & \multicolumn{2}{c|}{ Power Law After Apparent Break } \\
\hline Detector & $\begin{array}{c}\text { Frequency } \\
\text { Range } \\
{[\mathrm{Hz}]}\end{array}$ & $\begin{array}{c}\text { Photon } \\
\text { Index }\end{array}$ & $\chi^{2} /$ dof & $\begin{array}{c}\text { Frequency } \\
\text { Range } \\
{[\mathrm{Hz}]}\end{array}$ & $\begin{array}{c}\text { Photon } \\
\text { Index }\end{array}$ & $\chi^{2} / \mathrm{dof}$ \\
\hline HEAO A-1 & $0.1-3$ & $1.20 \pm 0.08$ & $15 / 13$ & $3-40$ & $1.7 \pm 0.2$ & $18 / 19$ \\
\hline RXTE/PCA & $0.1-3$ & $1.24 \pm 0.04$ & $28 / 14$ & $14-40$ & $2.1 \pm 0.5$ & $4 / 8$ \\
\hline
\end{tabular}

Table 1.1 Summary of power law fits to the noise-subtracted Fourier power spectrum. The breaks between the two effective power laws occur at $3 \mathrm{~Hz}$ and $10-20 \mathrm{~Hz}$ for the HEAO A-1 and RXTE/PCA data, respectively.

The RXTE/PCA data was Fourier analyzed in the same manner as the HEAO A-1 data in the attempt to both verify the HEAO A-1 results and resolve some of the uncertainties in those results that remained even after correcting for instrumental effects. The RXTE/PCA lacked evidence for any other instrumental effect except that due to dead time. Correcting the Poisson noise floor for dead time is discussed further in Chapter 4.

The resulting noise-subtracted power spectrum also shows two power laws in the spectrum as shown in Figure 1.14. The break between the two power laws is seen at a higher frequency between 10 to $20 \mathrm{~Hz}$, and features a more rounded shape. Table 1.1 summarizes the results of fitting power laws to the regions before and after this break.

The effect of approaching the Poisson noise floor is clearly demonstrated by the flattened spectrum at frequencies greater than $50 \mathrm{~Hz}$. It is an artifact introduced by limited counting statistics. The downward slope results from more statistics at higher frequencies. Despite the limiting statistics, evidence for marginal excess power is seen at frequencies between $100-4000 \mathrm{~Hz}$. Over $33 \%$ of the excess power at frequencies greater than $100 \mathrm{~Hz}$ exceeds the $95 \%$ confidence level curve.

No evidence is seen in this analysis of the Cyg X-1 data for the QPOs. A 95\% confidence level upper limit is shown in Figure 1.15 for detecting QPOs of a given fractional root-mean-square (rms) amplitude over a given logarithmic frequency interval. The QPOs for this limit are contained within a given logarithmic frequency interval, giving a quality factor, $\mathrm{Q}>9$. The best upper limits are obtained from RXTE/PCA 


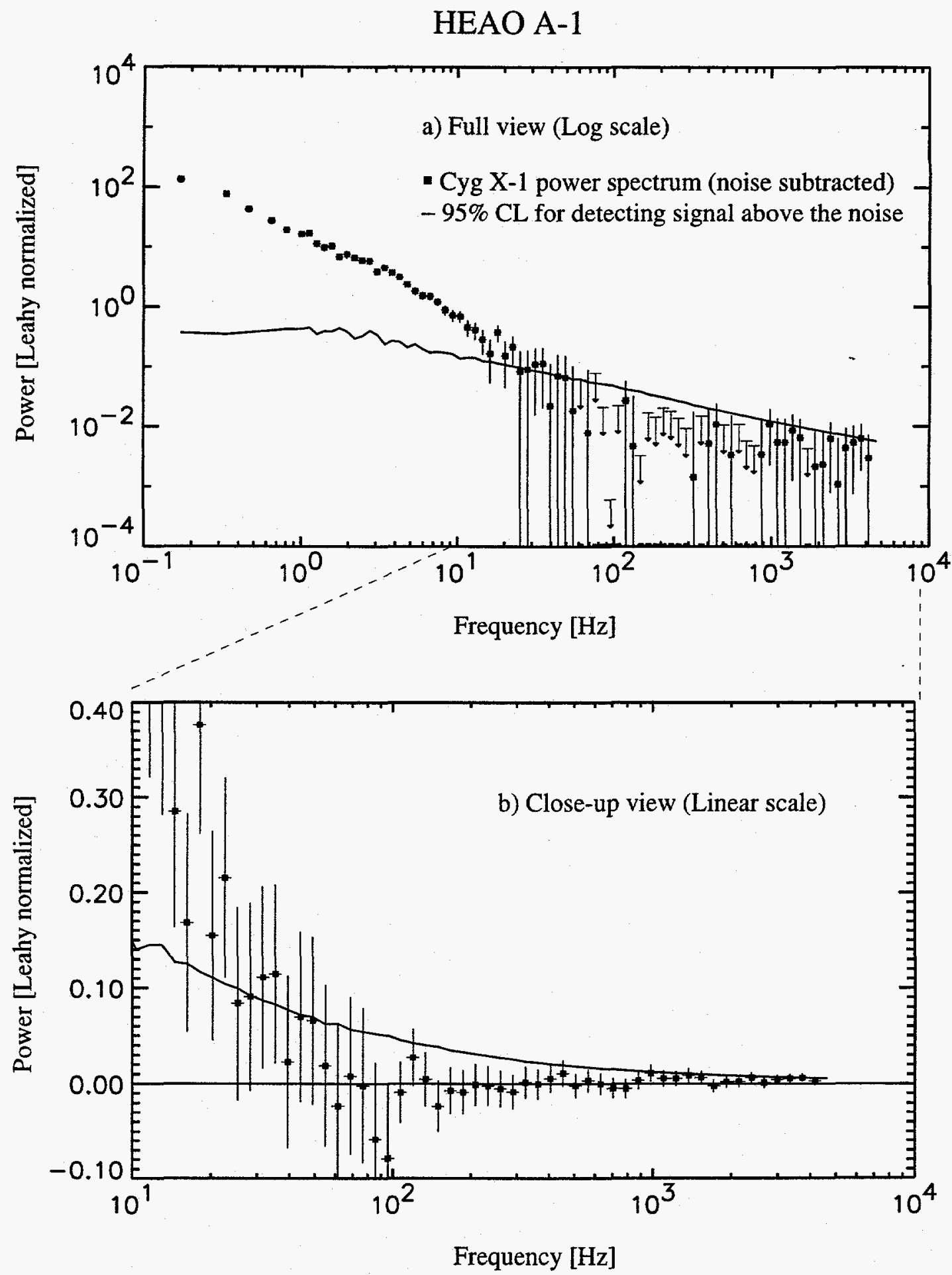

Fig. 1.13 The noise-subtracted Fourier power spectrum of Cygnus X-1 from the HBR data in a) full and b) close-up views. The Poisson noise floor was corrected for instrumental effects before the subtraction. The solid curve represents the $95 \%$ confidence level for detecting a signal above the Poisson noise floor. 


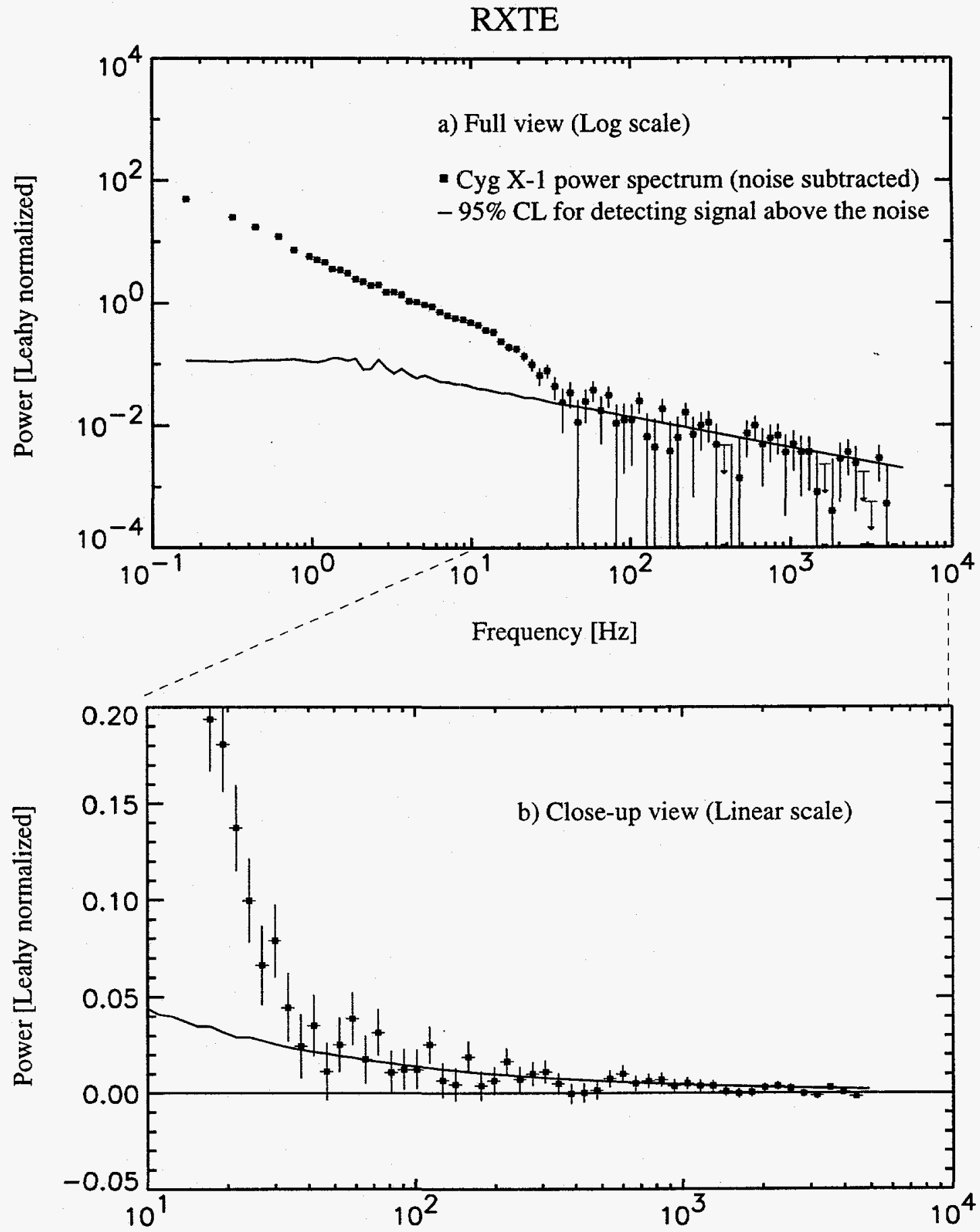

Frequency $[\mathrm{Hz}]$

Fig. 1.14 The noise-subtracted Fourier power spectrum of Cygnus X-1 from the RXTE/PCA data in a) full and b) close-up views. The Poisson noise floor was corrected for dead time before the subtraction. The solid curve represents the $95 \%$ confidence level for detecting a signal above the Poisson noise floor. 


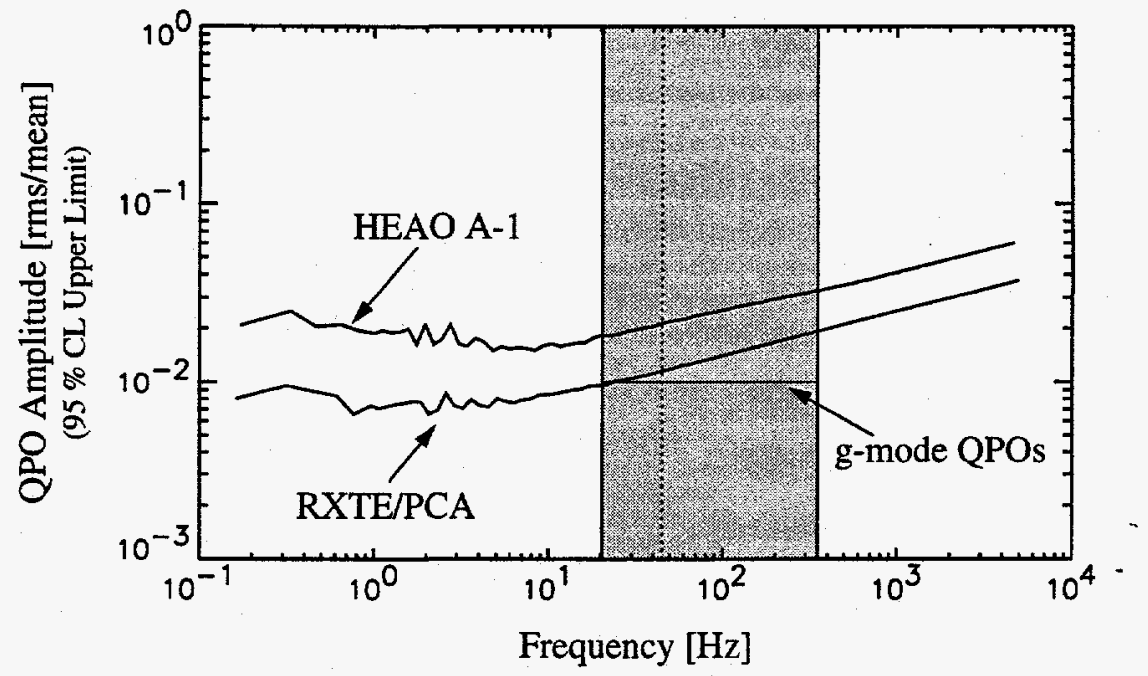

Fig. 1.15 The 95\% confidence level upper limit for detecting QPOs in Cyg X-1 with a quality factor, $Q>9$ for a given fractional rms amplitude. The shaded region spans the range of expected frequencies for g-mode QPOs, with the horizontal line marking the expected g-mode QPO amplitude and the dashed vertical line marking the frequency for a non-rotating, $16 M_{\odot}$ black hole.

since that observation contained six times more data than HEAO A-1 on Cyg X-1. Over the frequency range from 20.4 to $351 \mathrm{~Hz}$ where diskoseismic QPOs are predicted for Cyg $\mathrm{X}-1$, the fractional $\mathrm{rms}$ amplitudes are less than $1.9 \%$ at a $95 \%$ confidence level. For a $16 M_{\odot}$ black hole, the limit is $1.2 \%$. As Figure 1.15 shows, the $95 \%$ confidence level upper limit curve in the shaded region lies above the $\sim 1 \%$ fractional rms amplitude predicted for diskoseismic QPOs.

\subsection{Summary}

The study of X-ray time variability of close binary systems appears to be a promising method of searching for predicted black hole features. Evidence for a smooth drop-off may be interpreted from the faster fall off in variability at frequencies greater than the observed break from the $\sim 1 / f$ power law behavior. The break occurs in the HEAO A-1 and RXTE/PCA data at roughly $3 \mathrm{~Hz}$ and between $10-20 \mathrm{~Hz}$, respectively. Both breaks occur at frequencies within the range of drop-offs expected from the advection-dominated TCAF model. The break between $10-20 \mathrm{~Hz}$ is also near the sharp 
rollover predicted by Nowak and Wagoner's model of accretion disk turbulence. More RXTE/PCA data is needed to determine how the drop-off behaves at frequencies greater than $50 \mathrm{~Hz}$, by lowering the expected variance in the Poisson noise floor (the solid curve in Figure 1.14). As Figure 1.16 shows, the noise-subtracted spectra from the HEAO A-1 and RXTE/PCA data are consistent with each other within their given error bars at frequencies greater than $\sim 10 \mathrm{~Hz}$. The drop-off starting at $3 \mathrm{~Hz}$ in the HEAO A-1 data matches smoothly to the start of the drop-off at $\sim 10 \mathrm{~Hz}$ in the RXTE/PCA data.

At frequencies less than $10 \mathrm{~Hz}$, the variability in the HEAO A-1 data is 2-3 times greater than the RXTE/PCA data with the same approximate $\sim 1 / f$ power law behavior. These differences may be attributed in part to the different states Cyg X-1 was in for the two periods of observations: low state for HEAO A-1 and high state for RXTE/PCA. In addition, the two instruments have different detector efficiences and are thus sensitive to detecting X-rays in different energy ranges. As described in Chapter 2, the HEAO A-1 and RXTE/PCA detectors are sensitive to detecting photons at $15 \%$ efficiency with energies of $1 \mathrm{keV}$ and $2.5 \mathrm{keV}$, respectively. Assuming a power law with a spectral index of 1.5 , the photons from $1-2.5 \mathrm{keV}$ comprise about $17 \%$ of the total number of photons detected by HEAO A-1. Unfortunately, individual photon energy information was not available to the HEAO A-1 detector. The USA detector, which is sensitive to detecting $1.1 \mathrm{keV} \mathrm{X}$-rays at $15 \%$ efficiency will be able to test if photons from $1-2.5 \mathrm{keV}$ exhibit greater variability than photons with energies greater than 2.5 $\mathrm{keV}$.

The locations of the apparent breaks in the two power spectra occur at frequencies too low to be evidence of drop-offs near the marginally stable orbit. Instead, they may indicate the location of the shock formed from advection-dominated accretion or for the HEAO A-1 data, the outer edge of the Compton cloud.

The interpreted drop-offs exhibit a lower degree of steepness than predicted by

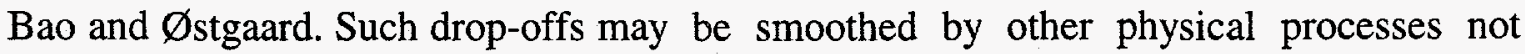
explicitly included in their model. The X-ray emissions from Cyg X-1 may suffer from interference from a Compton cloud or hot corona believed to surround the black hole. In 


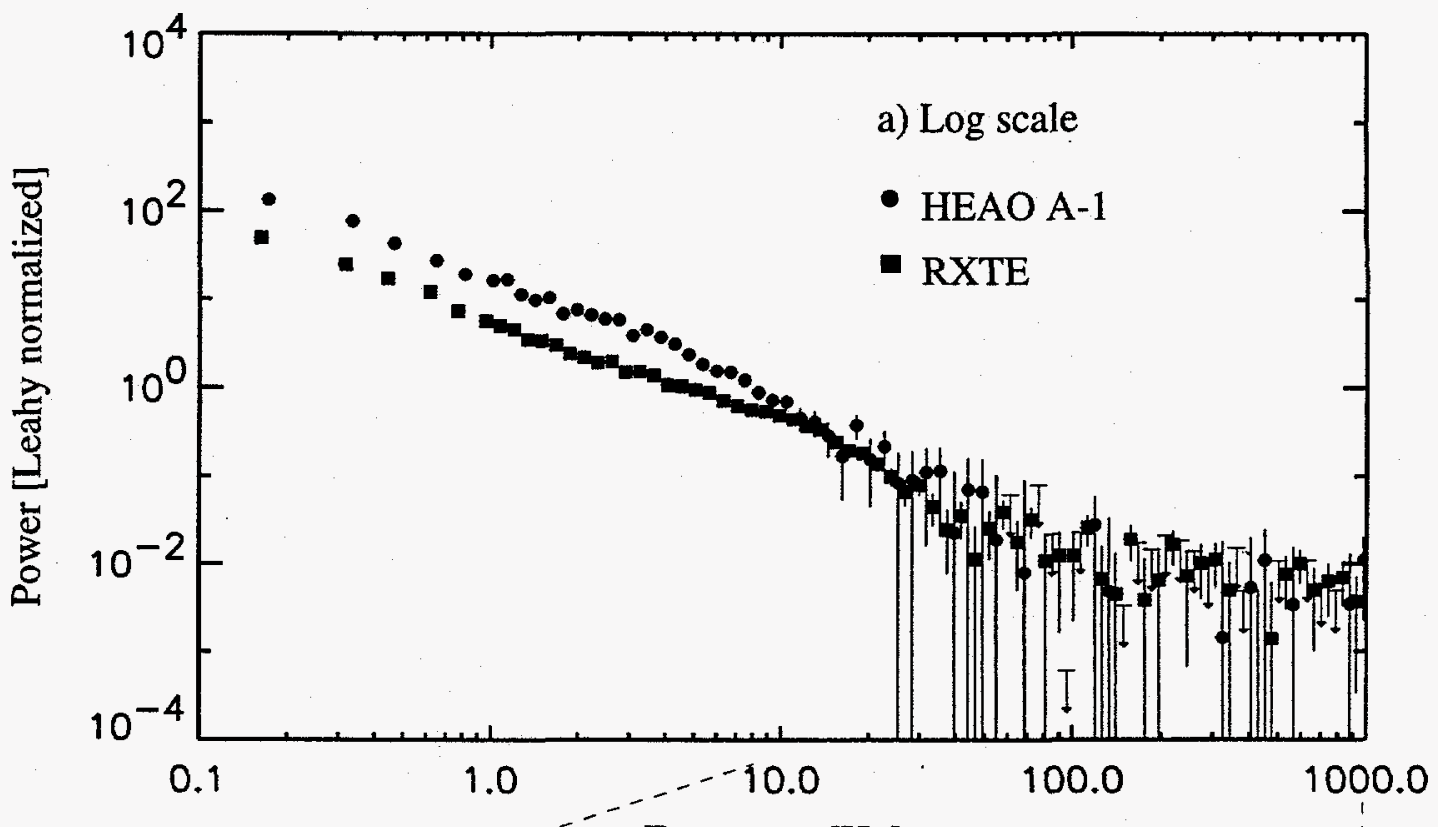

Frequency [Hz]

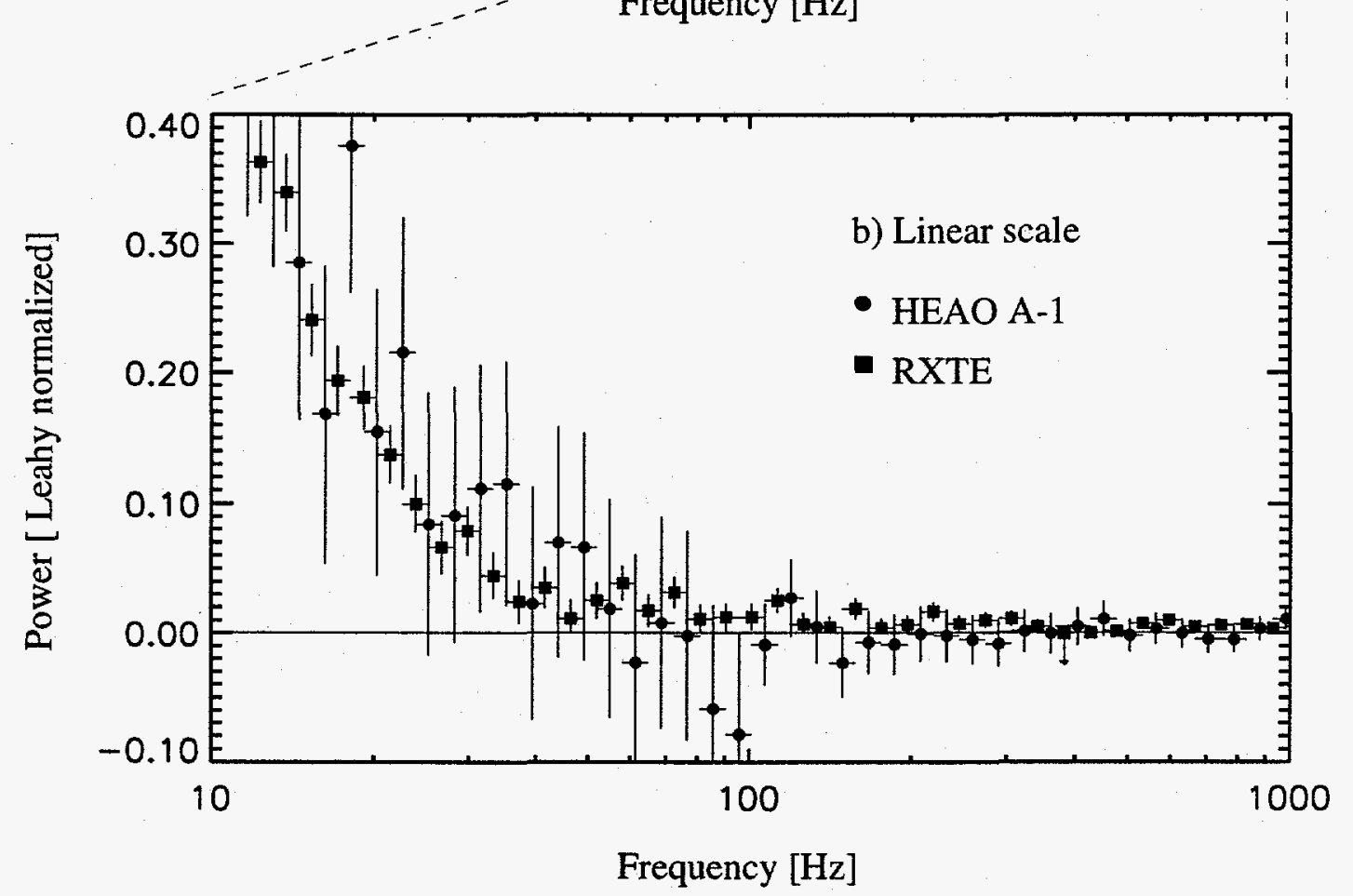

Fig. 1.16 The noise-subtracted Fourier power spectra of Cygnus X-1 from the HEAO A-1 and RXTE/PCA data shown together a) from 1-100 Hz on a $\log$ scale and b) from $10-100 \mathrm{~Hz}$ on a linear scale. The two spectra are consistent with each other within their given error bars for frequencies greater than $\sim 10 \mathrm{~Hz}$. 


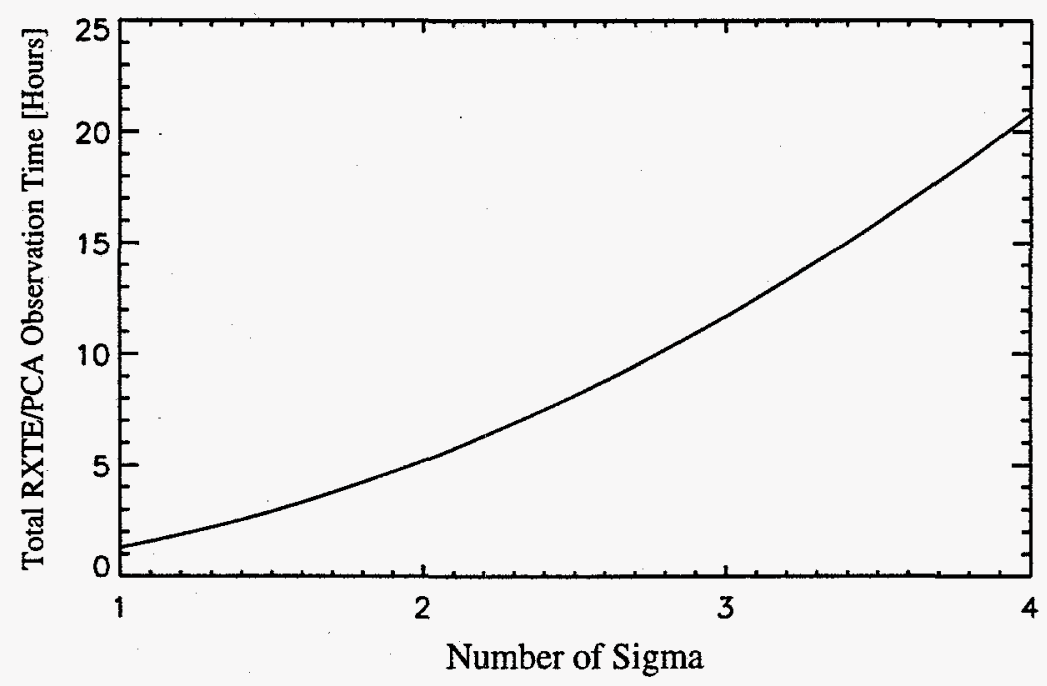

Fig. 1.17 Total integrated RXTE/PCA observation time required to detect diskoseismic QPOs in Cyg X-1 with a Fourier power amplitude a given number of sigma above Poisson noise. A fractional rms amplitude $\sim 1 \%$ and quality factor, $\mathrm{Q}>9$ are assumed for the QPOs.

the alternative advection-dominated disk model, the shock formed by sub-Keplerian flows can strongly interfere with the X-ray emissions near the inner edge of the accretion disk.

Given the amount of Cyg X-1 data analyzed from RXTE/PCA, diskoseismic QPOs with $1 \%$ fractional rms amplitudes can be excluded for frequencies $\leq 25 \mathrm{~Hz}$ at a 95\% confidence level. The excluded frequencies contained within the shaded region in Figure 1.15 correspond to retrograde orbits with black hole angular momenta parameter $a \leq-0.5$. As discussed in Chapter 3, the QPO amplitude is proportional to the square root of the standard error of the average Fourier power. This standard error as described in Section 1.2 is inversely proportional to the square root of the number of data segments averaged, namely, the length of the data. Therefore, the QPO amplitude is inversely proportional to the fourth root of the length of data. A $95 \%$ confidence level upper limit for detecting diskoseismic QPOs in Cyg X-1 at a 1\% fractional rms amplitude over the entire range of predicted frequencies, requires a total of five hours of integrated RXTE/PCA observation time. 
If we instead assume that diskoseismic QPOs are present in Cyg X-1, then an important experimental quantity is the amount of data needed to detect the QPOs at a given confidence level above the Poisson noise. Figure 1.17 shows the total integrated RXTE/PCA observation time required to detect diskoseismic QPOs with a Fourier power amplitude a given number of sigma above Poisson noise. A quality factor, $Q>9$ and a1\% fractional rms amplitude over the range of predicted frequencies were assumed. To detect 4-sigma diskoseismic QPOs in Cyg X-1, a total integrated RXTE/PCA observation time of 21 hours or about a day is required. 


\section{References}

1) Bowyer, S., Byram, E.T., Chubb, T.A. \& Friedman, H. (1965), Science, 147, 394.

2) Gies, D.R. \& Bolton, C.T. (1982), ApJ, 260, 240; (1986), ApJ, 304, 371.

3) Dolan, J. F., Crannell, C. J., Dennis, B. R., Frost, K. J., \& Orwig, L.E. (1979), ApJ, 230, 551.

4) Tanaka, Y. \& Lewin, W.H.G. (1995), X-Ray Binaries, W.H.G. Lewin, J. V. Paradijs and E.P.J. Van den Heuvel, editors, Cambridge University Press, Cambridge, England, p. 131.

5) Meekins, J. F., Wood, K. S., Hedler, R. L., Byram, E. T., Yentis, D. J., Chubb, T. A., \& Friedman, H.. (1984), ApJ, 278, 288.

6) Margon, B., Bowyer, S., \& Stone, R.P.S. (1973), ApJ Lett. 185, L113; Bregman, J., Butler, D., Kemper, E., Koski, A., Kraft. R.P., \& Stone, R.P.S. (1973), ApJ Lett. 185, L117.

7) Zhang, S. N., Cui, W., Harmon, B. A., Paciesas, W. S., Remillard, R. E., \& Paradijs, J. V. (1997), ApJ Lett. 477, L95; Cui, W., Heindl, W. A., Rothschild, R. E., Zhang, S. N., Jahoda, K., \& Focke, W. (1997), ApJ Lett. 474, L57; Belloni, T., Mendez, M., Van der Klis, M., Hasinger, G., Lewin, W.H.G., Paradijs, J. V. (1996), ApJ Lett. 472, L107; Dotani, T., Negoro, H., Mitsuda, K., Inoue, H., \& Nagase, F. (1996), IAU Circ. 6415.

8) Schwarzschild, K. (1916), Sitzungsberichte der Deutschen Akademie der Wisenschaften zu Berlin, Klasse fur Mathematik, Physik, und Technik, 189.

9) Misner. C. W., Thorne, K. S., \& Wheeler, J. A. (1973), Gravitation, W. H. Freeman: New York.

10) Kerr, R. P. (1963), Phys. Rev. Lett., 11, 237.

11) Boyer, R. H. \& Lindquist, R. W. (1967), J. Math. Phys. 8, 265.

12) Bardeen, J. M., Press, W. H., and Teukolsky, S. A. (1972), ApJ, 178, 347. 
13) Shapiro, S. L. \& Teukolsky, S. A. (1983), Black Holes, White Dwarfs, and Neutron Stars: The Physics of Compact Objects, J. Wiley: New York.

14) Shakura, N. I. \& Sunyaev, R. A. (1973), Astron. Astrophys. 24, 337.

15) Novikov, I. D. \& Thorne, K. S. (1973), Black Holes, C. DeWitt and B. DeWitt, editors, Gordon and Breach, New York, New York.

16) Thorne, K. S. \& Price, R. H. (1975), ApJ Lett. 195, L101.

17) Ostriker, J. P. (1976), ApJ, 218, 243.

18) Liang, E.P.T. \& Price, R. H. (1977), ApJ, 218, 247.

19) Bisnovatyi-Kogan, G. S. \& Blinnikov, S. I. (1977), Astron. Astrophys. 59; 111.

20) Chakrabarti, S. K. \& Titarchuck, L. G. (1996), ApJ, 455, 623; Chakrabarti, S. K. \& Molteni, D. (1995), MNRAS, 272, 80; Chakrabarti, S. K. (1996a), Phys. Rep., 266, 229; (1996b), ApJ, 464, 664 .

21) Narayan, R. (1996), ApJ, 462, 136; Narayan, R., et al. (1995), ApJ, 444, 231; Narayan, R., et al. (1995), ApJ, 452, 710.

22) Eddington, A. S. (1926), The Internal Constitution of the Stars, Cambridge University Press, Cambridge, England.

23) Bao, G. \& Østgaard, E. (1995), ApJ, 443, 54; Bao, G. (1992), A\&A, 257, 594; Bao, G., Hadrava, P., \& Østgaard, E. (1994a), ApJ, 425, 63.

24) Nowak, M. A. \& Wagoner, R. V. (1995), MNRAS, 274, 37.

25) Kato, S. \& Fukue, J. (1980), PASJ, 32, 377.

26) Nowak, M. A. \& Wagoner, R. V. (1991), ApJ, 378, 656; (1992), ApJ, 393, 697; (1993), ApJ, 418, 187; (1995), MNRAS, 274, 37; Nowak, M. A., Wagoner, R. V., Begelman, M. C., \& Lehr, D. E. (1997), ApJ, 477, L91.

27) Perez, C. A., Silbergleit, A.S., Wagoner, R. V., \& Lehr, D. E. (1997), ApJ, 476, 589.

28) Ipser, J. R. \& Lindblom, L. (1992), ApJ, 389, 392.

29) Terrell, N. J. (1972), ApJ, 174, L35; Lochner, J. C., Swank, J. H., \& Szymkowiak, A. E. (1991), ApJ, 376, 295.

30) Bak, P., Tang, C., \& Wiesenfeld, K. (1988), Phys. Rev. A, 38, 364.

31) Mineshige, S., Ouchi, N. B., \& Nishimori, H. (1994), PASJ, 46, 97; (1994), ApJ, 435, L125.

32) Rhoades, C. E. \& Ruffini, R. (1974), Phys Rev Lett. 32, 324.

33) Baym, G., Pethick, C. \& Sutherland, P. (1971b), ApJ, 170, 299. 
34) Bloom, E. B. (1994), Particle Physics, Astrophysics \& Cosmology, Proceedings of the XXII SLAC Summer Institute on Particle Physics, SLAC-Report-484, p. 133.

35) Mitsuda, K. et al. (1984), PASJ, 36, 741.

36) Lewin, W.H.G. \& Joss, P.C. (1981), Space Sci. Rev. 28, 3; (1983), Accretion Driven Stellar X-ray Sources, W.H.G. Lewin and E.P.J. Van den Heuvel, editors, Cambridge University Press, Cambridge, England, p. 41.

37) Hoyle, F., Narlikar, J.V., \& Wheeler, J.A. (1964), Nature, 203, 914; Tsuruta, S. \& Cameron, A.G.W. (1966), Nature, 211, 356; Woltjer, L. (1964), ApJ, 140, 1309; Wheeler, J. A. (1966), Ann. Rev. Astron. Astrophys. 4, 393.

38) Leahy, D. A., Darbro, W., Elsner, R. F., Weisskopf, M. C., Sutherland, P. G., Kahn, S., \& Grindlay, J. E. (1983), ApJ, 266, 160. 
Chapter 2

SUMMARY OF X-RAY MISSIONS

\subsection{Introduction}

$\mathrm{X}$-ray time variability characteristics unique to black hole binary systems are expected at frequencies spanning several orders of magnitude depending on the properties of the black hole and the accretion disk. For Cyg X-1, the frequency range spans from tens of Hertz to several kiloHertz. A search for such variability characteristics in Cyg X-1 requires an instrument with time resolution of 50 microseconds or better. Three astronomical instruments that have flown, are flying or will soon fly currently satisfy this requirement, the High Energy Astrophysical Observatory (HEAO-1), the Rossi X-ray Timing Explorer (RXTE) and the Unconventional Stellar Aspect Experiment (USA). All three of these instruments have 10 microsecond time resolution capabilities or better. This Chapter will describe these instruments in greater detail.

\subsection{High Energy Astrophysical Observatory (HEAO-1)}

\subsubsection{Mission}

The first of NASA's three High Energy Astrophysical Observatories, HEAO-1 was launched aboard an Atlas Centaur rocket on 12 August 1977 and operated until 9 January 1979. During that time, it scanned the X-ray sky almost three times in 


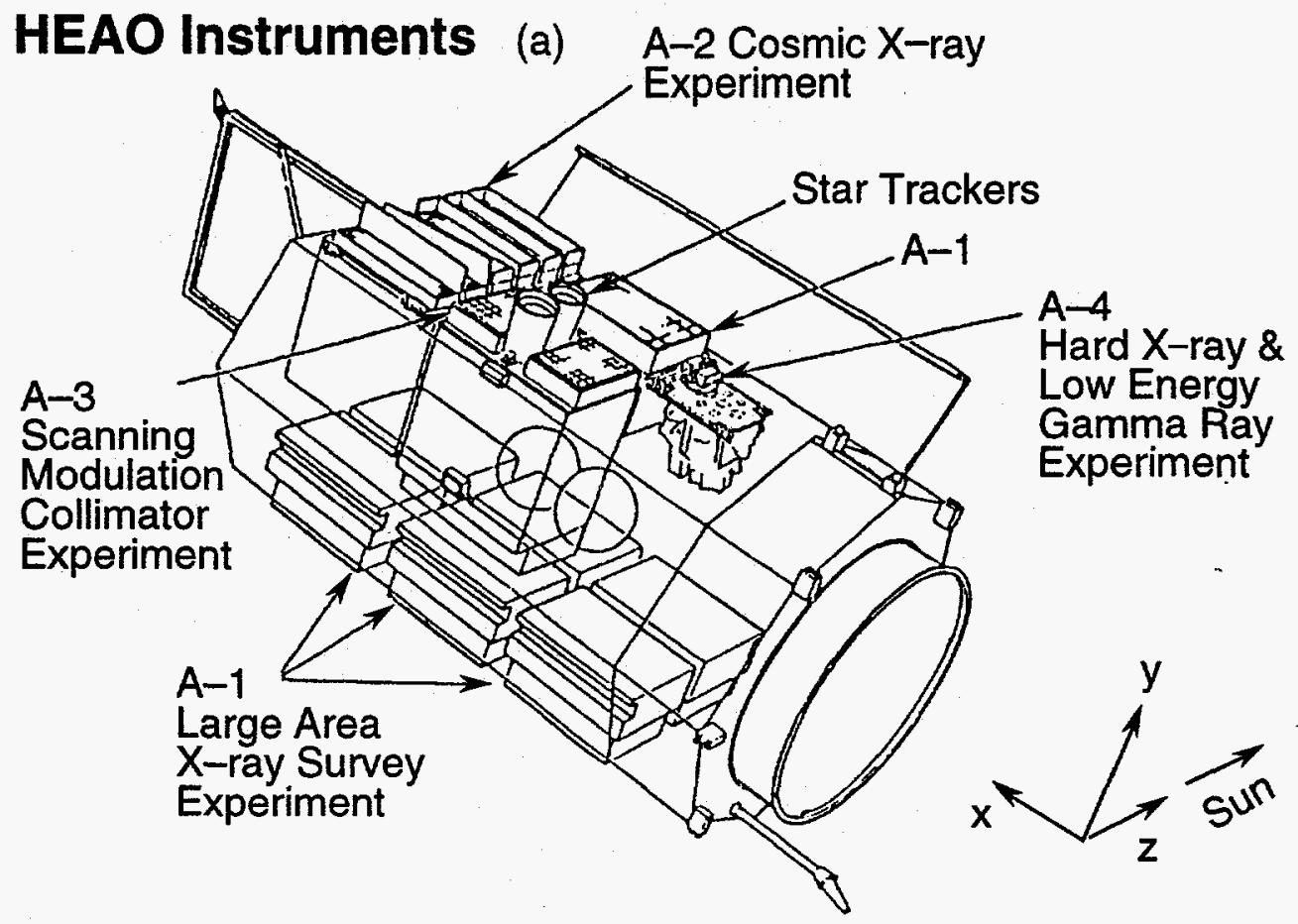

\section{NRL \& NASA (b) \\ 1977-79}

HEAO A-1

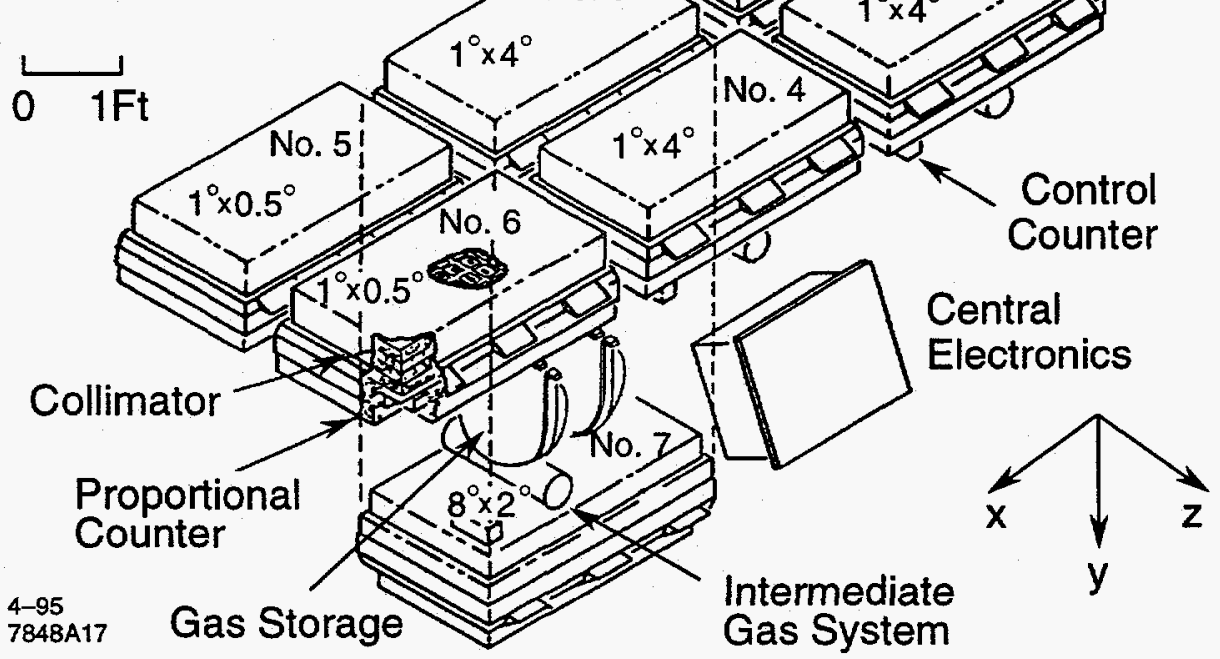

Fig. $2.1 \quad$ a) The High Energy Astrophysical Observatory-1 HEAO-1 satellite shown with its four major experiments identified. b) An exploded view of the HEAO A-1 detector. 


\begin{tabular}{|c|c|c|}
\hline Name & Mission & Instrumentation \\
\hline $\begin{array}{l}\text { A1 - Large Area Sky } \\
\text { Survey experiment } \\
(\text { LASS })^{[1]}\end{array}$ & All-sky survey of X-ray sources & $\begin{array}{l}\text { Array of seven proportional } \\
\text { counters with } 1350 \mathrm{~cm}^{2}, 1650 \\
\mathrm{~cm}^{2} \text { and } 1900 \mathrm{~cm}^{2} \text { open areas } \\
\text { covering } 0.25 \text { to } 25.0 \mathrm{keV} \text {. }\end{array}$ \\
\hline $\begin{array}{l}\text { A2 - Cosmic X-ray } \\
\text { Experiment }(\mathrm{CXE})^{[2]}\end{array}$ & $\begin{array}{l}\text { Study the large scale structure } \\
\text { of the galaxy and the universe } \\
\text { (diffuse X-ray background) }\end{array}$ & $\begin{array}{l}\text { Array of six proportional } \\
\text { counters with } 400 \mathrm{~cm}^{2} \text { and } 800 \\
\mathrm{~cm}^{2} \text { open areas covering } 0.15- \\
60 \mathrm{keV}\end{array}$ \\
\hline $\begin{array}{l}\text { A3 - Modulation } \\
\text { Collimator }(\mathrm{MC})^{[3]}\end{array}$ & $\begin{array}{l}\text { Measure the positions of X-ray } \\
\text { sources with sufficient } \\
\text { precision to identify optical } \\
\text { and/or radio counterparts. }\end{array}$ & $\begin{array}{l}\text { Two four-grid modulation } \\
\text { collimators with } 300 \mathrm{~cm}^{2} \\
\text { effective area covering } 0.9- \\
13.3 \mathrm{keV} \text {. Positional accuracy } \\
\text { was projected to be } \sim 2 \text {. }\end{array}$ \\
\hline $\begin{array}{l}\text { A4 - Hard X-Ray / } \\
\text { Low Energy Gamma } \\
\text { Ray Experiment }^{[4]}\end{array}$ & $\begin{array}{l}\text { Observe hard X-rays } \\
\text { / low-energy gamma-rays }\end{array}$ & $\begin{array}{l}\text { Seven scintillators with } 45 \mathrm{~cm}^{2} \\
\text { and } 100 \mathrm{~cm}^{2} \text { areas covering } 15 \\
\mathrm{keV}-10 \mathrm{MeV} \text {. }\end{array}$ \\
\hline
\end{tabular}

Table 2.1 Brief summary of the four experiments in HEAO 1.

the energy range $0.2 \mathrm{keV}-10 \mathrm{MeV}$, provided nearly constant monitoring of X-ray sources near the ecliptic poles, as well as more detailed studies of a number of objects through pointed observations. HEAO-1 was primarily a survey mission, dedicated to systematically mapping the X-ray sky every 6 months. The satellite was launched into a nearly circular orbit with apogee $445 \mathrm{~km}$ and inclination $22.75^{\circ}$. HEAO-1 had a 93 minute orbital period, and, while in scanning mode, spun with a nominal period of 33 minutes. Each spin traced out a great circle of constant ecliptic longitude. Every twelve hours, the spin axis was moved approximately $0.5^{\circ}$ in order to keep it pointed at the Sun; thus, after 6 months, the entire sky had been observed. After the first $\sim 100$ days of the mission, scanning was interrupted from time to time to point the detectors at particular objects of interest. These pointing times became more frequent until 9 January 1979, when the gas used to control the spacecraft attitude ran out. The systems were shutdown, and HEAO-1 drifted in a decaying orbit until March 1979, when it burned up 


\begin{tabular}{|c|c|c|c|l|}
\hline $\begin{array}{c}\text { Sensor } \\
\text { Module }\end{array}$ & FWHM & $\begin{array}{c}\text { View } \\
\text { Direction }\end{array}$ & $\begin{array}{c}\text { Open Area } \\
\left(\mathrm{cm}^{2}\right)\end{array}$ & \multicolumn{1}{|c|}{ Comments } \\
\hline 1 & $1^{\circ} \times 4^{\circ}$ & $-Y$ & 1650 & Failed 9/23/77 \\
\hline 2 & $1^{\circ} \times 4^{\circ}$ & $-Y$ & 1650 & Failed 9/22/77 \\
\hline 3 & $1^{\circ} \times 4^{\circ}$ & $-Y$ & 1650 & Single Detector Case \\
\hline 4 & $1^{\circ} \times 4^{\circ}$ & $-Y$ & 1650 & Failed $1 / 26 / 78$ \\
\hline 5 & $1^{\circ} \times 1 / 2^{\circ}$ & $-Y+1 / 3^{\circ} Z$ & 1350 & Not used in this analysis \\
\hline 6 & $1^{\circ} \times 1 / 2^{\circ}$ & $-Y-1 / 3^{\circ} Z$ & 1350 & \\
\hline 7 & $2^{\circ} \times 8^{\circ}$ & $+Y$ & 1900 & Intermittent severe noise \\
\hline
\end{tabular}

Table 2.2 Summary of HEAO A-1 Module properties. Modules 5 and 6 were tilted a third of a degree either towards or away from the $\mathrm{Z}$ (Sun-pointed) axis.

on re-entry into the atmosphere.

\subsubsection{Instrumentation}

HEAO-1 carried four instruments all used primarily in a scanning mode, with a small number of pointed observations. Table 2.1 briefly describes each of these four experiments. Of special interest is the A1 instrument, also known as the NRL Large Area Sky Survey Experiment (LASS) or HEAO A-1 ${ }^{[1]}$.

As Figure 2.1 shows, the HEAO A-1 instrument consisted of seven sensor modules, six mounted on the $-Y$ side of the spacecraft, the seventh on the $+Y$ side. Each sensor module had a collimator constructed of rectangular molybdenum tubes of various dimensions. Table 2.2 summarizes their properties. The experiment had sufficient sensitivity to detect sources as faint as $0.25 \mu \mathrm{Jy}$ at $5 \mathrm{keV}$ for sources with a Crab-like spectrum. Data was typically collected in either a 5 or a 320 millisecond timing resolution mode. Full sky coverage for both time resolutions was achieved before the mission's end.

Each sensor module consisted of a $2^{\prime} \times 3^{\prime}$ proportional counter body frame on which was mounted support structures, a collimator assembly and electronic subassemblies. 


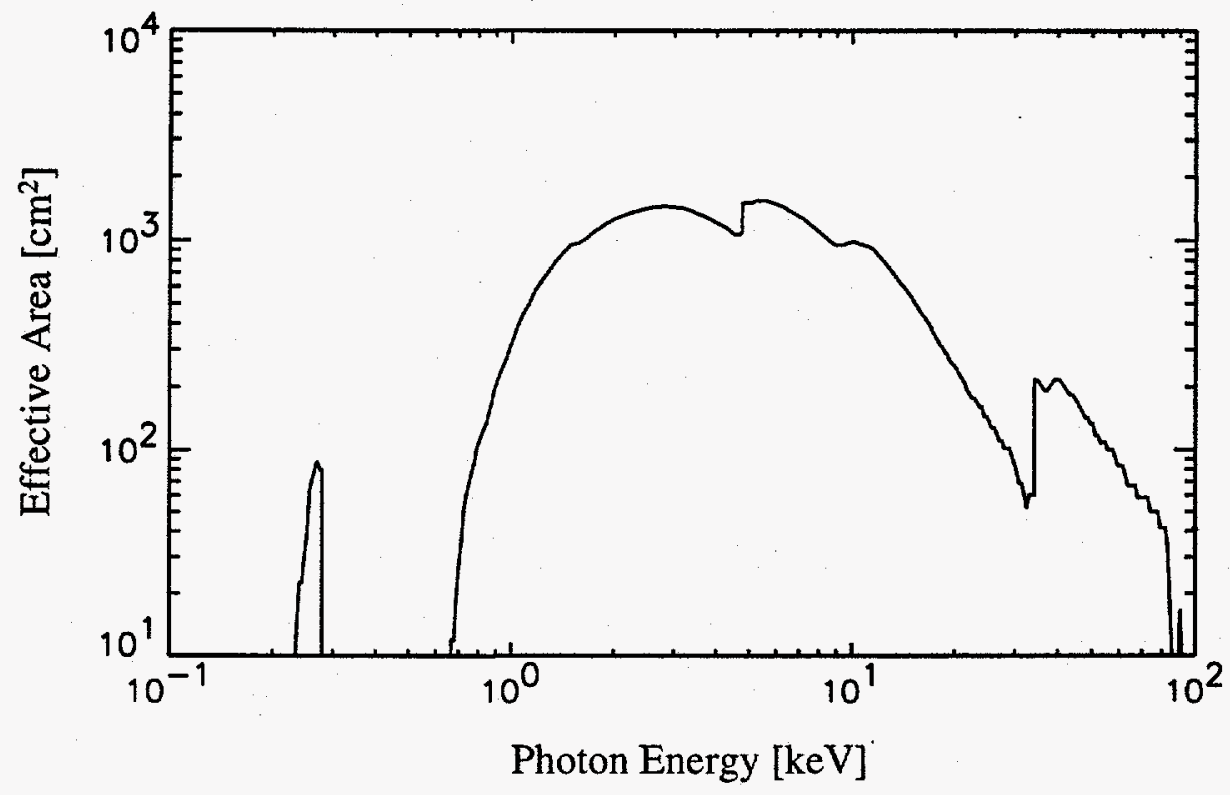

Fig. 2.2 HEAO A-1 effective area for a single Module (1,2,3 or 4$)$ for the sum of both wire layers as a function of photon energy. Cyg X-1 data used in this analysis collected from Module 3 only.

The proportional chamber contained three wire layers, where the top layer usually served as the X-ray sensor while the lower two layers provided anticoincidence protection against charged particle events. Additional anticoincidence protection was provided on the ends and sides.

The gas in the chamber was supplied by a central gas system consisting of two 524 psi spherical tanks filled with a gas mixture of $77.5 \%$ Xenon and $22.5 \%$ Methane. Each tank was separately valved to a gas distribution system, supplying gas to any or all of the seven sensor modules.

A $2.5 \mu \mathrm{m}$ layer of mylar contained the gas volume. To maintain electrical conductivity on the inward surface, the mylar was coated with a thin film of Nichrome, $45 \AA$ thick. In addition to the collimator face, a stainless steel wire mesh was placed in-between the collimator and the mylar window to provide additional support and protect against any small burrs on the collimator face. To protect against overheating from the sun, the collimator was covered with a $2 \mu \mathrm{m}$ aluminized Kimfol heat shield that had an 
effective transmission of $91.2 \%$. Figure 2.2 shows the resulting effective $X$-ray acceptance for HEAO A-1 module 1, 2, 3 or 4 .

Each sensor module had its own independent electronics consisting of preamplifiers, high voltage filter and logic and digitizing circuitry. The circuitry was composed of a remote serial magnitude command decoder, a data analog-to-digital converter and a housekeeping multiplexer.

Processing the digitized data from the modules was a task left to the central electronics. It sorted the module data according to either a 16-channel or a 40 -channel nonlinear energy scale and routed it to scalar accumulators. Depending on the telemetry format chosen (320 msec/5 msec) additional processing may have been applied to the data with the accumulated counts saved into the appropriate data format.

One special telemetry mode consisted of utilizing the Random Encoder unit to transmit events at a $1-8 \mu$ s time resolution in real time at a high-bit-rate (HBR) of 128 kps. Events received originated from either the sum of sensor modules 1-4 or singly from module 7 , depending on which side of the satellite the $\mathrm{X}$-ray source was located. Since modules 1, 2 and 4 had failed by the time most of these HBR telemetry modes were commanded, most of the resulting data in this mode originated only from either sensor module 3 or 7 .

\subsection{Rossi X-Ray Timing Explorer (RXTE)}

\subsubsection{Mission}

The Rossi X-ray Timing Explorer is a NASA mission with instruments built by the NASA Goddard Space Flight Center (GSFC), Massachusetts Institute of Technology (MIT) and the University of California, San Diego (UCSD) that was launched on December 30, 1995. RXTE is designed to facilitate the study of time variability in the emission of X-ray sources with moderate spectral resolution. Time scales from microseconds to months are covered in an instantaneous spectral range from 2 to 250 $\mathrm{keV}$. It is designed for a required lifetime of two years, with a goal of five years. 


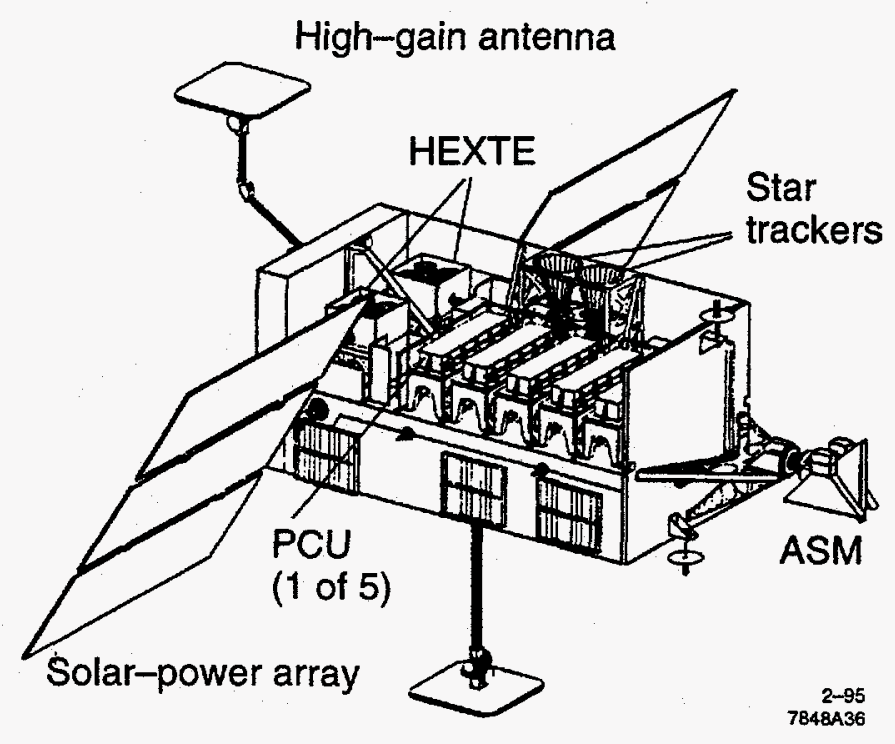

Fig. 2.3 The Rossi X-Ray Timing Explorer satellite.

The spacecraft was designed and built by the Engineering Directorate at GSFC. The launch vehicle was a Delta II rocket that put RXTE into a low-earth circular orbit at an altitude of $580 \mathrm{~km}$, corresponding to an orbital period of about 90 minutes, with an inclination of $22.75^{\circ}$.

\subsubsection{Instrumentation}

The mission carries two pointed instruments, the Proportional Counter Array $(\mathrm{PCA})^{[5]}$ developed by GSFC to cover the lower part of the energy range, and the High Energy X-ray Timing Experiment (HEXTE) ${ }^{[6]}$ developed by UCSD covering the upper energy range. These instruments shown in Figure 2.3 are equipped with collimators yielding a $1^{\circ}$ FWHM. RXTE also carries an All-Sky Monitor (ASM) ${ }^{[7]}$ from MIT that scans about $80 \%$ of the sky every orbit, allowing monitoring at time scales of 90 minutes or longer. Data from the PCA and the ASM are processed on board by the Experiment Data System (EDS) ${ }^{[8]}$, also built by MIT.

The PCA is an array of five proportional counters shown in Figure 2.4 with a total collecting area of $6500 \mathrm{~cm}^{2}$. Sensitive to a spectral range from $2-60 \mathrm{keV}$ at an energy resolution $<18 \%$ at $6 \mathrm{keV}$, the $\mathrm{X}$-rays can be recorded to 1 microsecond resolution. The effective X-ray acceptance for RXTE/PCA is shown in Figure 2.5. Each PCA unit has a 
a) PCA Assembly (5 units)

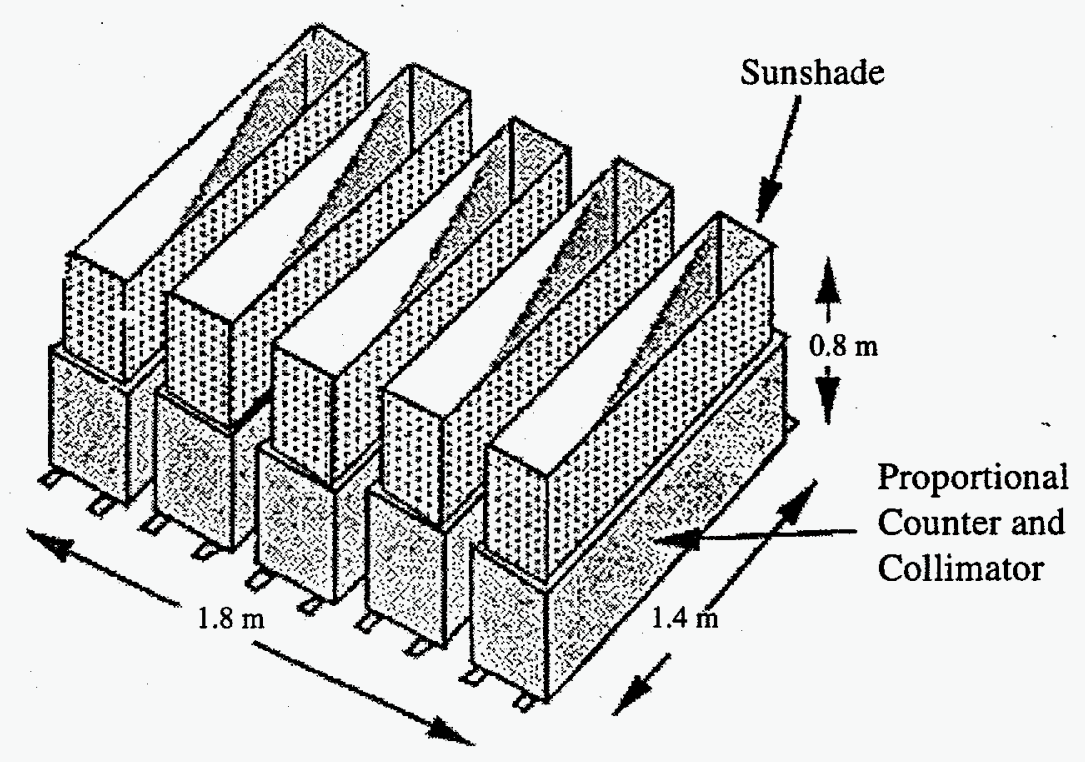

b) Proportional Counter (1 unit)

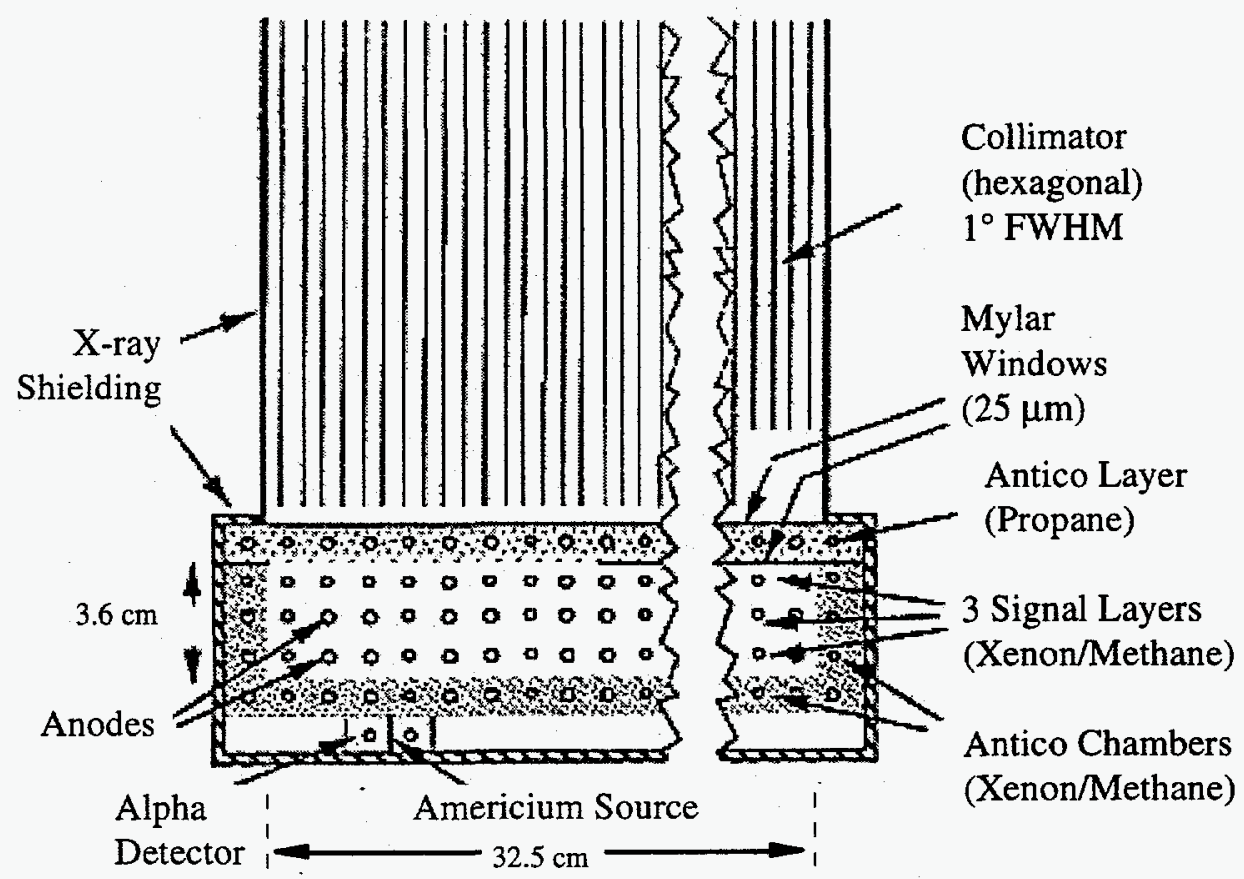

Fig. 2.4 a) Layout of the Proportional Counter Array (PCA). b) Cross section of a PCA unit showing the various components of its operation. 


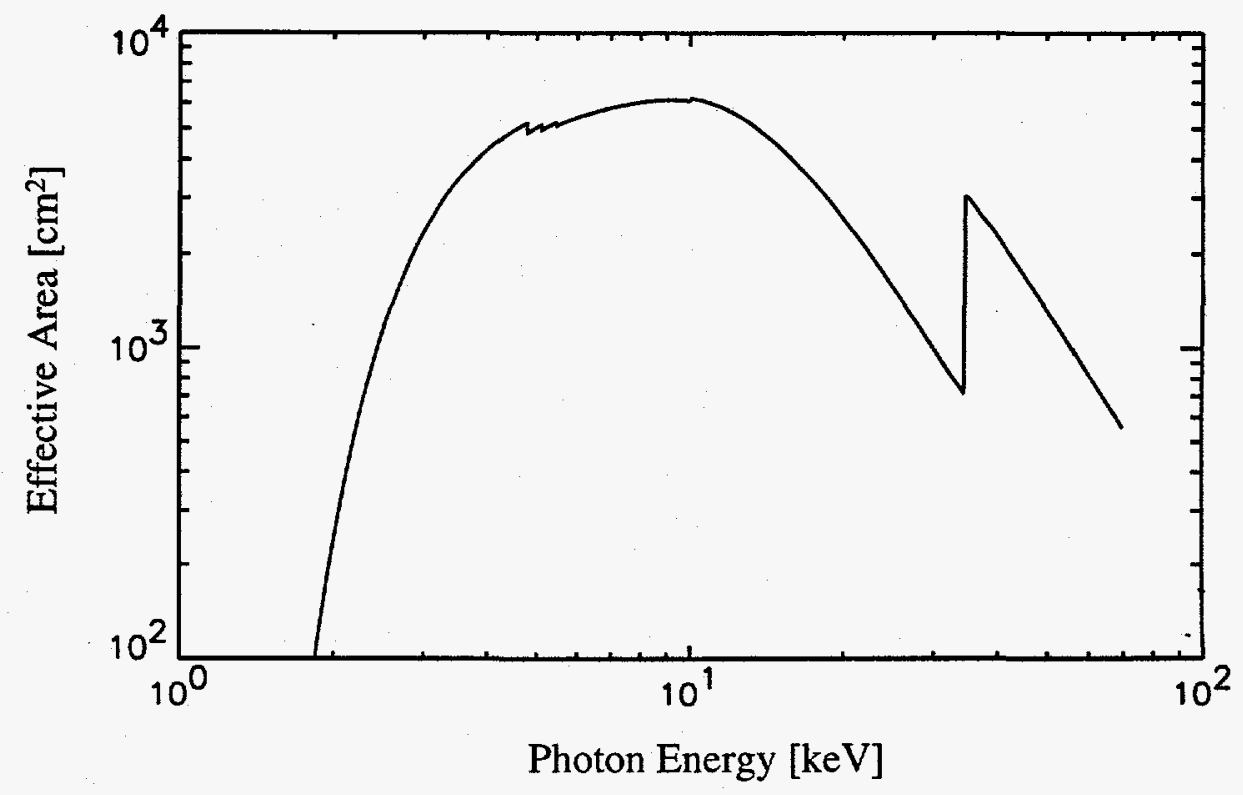

Fig. 2.5 RXTE/PCA effective area for all PCUs and all anodes as a function of photon energy.

collimator constructed of a honeycomb of hexagonal tubes with $1^{\circ}$ FWHM. An additional "single-cell" rectangular tube sits on top of the collimator to serve as a sun shade.

Sitting below the collimator, a thin proportional chamber is filled with propane gas and netted down its center with a single layer of wires to serve as a anticoincidence layer. Below this thin chamber is the main proportional chamber surrounded along its perimeter by anticoincidence chambers with a single layer of wires running through. The main proportional chamber contains three signal layers and shares a mixture of $78 \%$ Xenon and 22\% Methane gas with the anticoincidence chambers. To contain the propane gas in the anticoincidence layer, two layers of $25 \mu \mathrm{m}$ thick mylar are positioned, one above and the other below the layer's boundaries. Figure 2.4 summarizes the components of this PCA unit. 


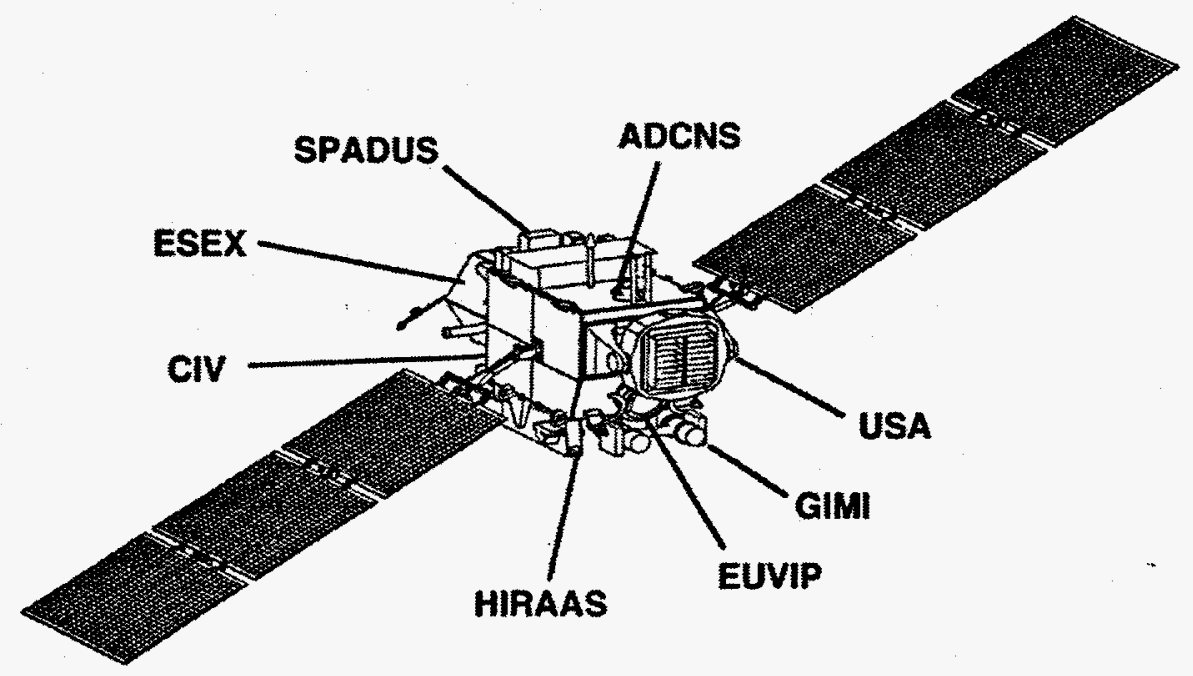

Fig. 2.6 The Advanced Research and Global Observation (ARGOS) satellite.

\subsection{Unconventional Stellar Aspect Experiment (USA)}

\subsubsection{Mission}

The Unconventional Stellar Aspect Experiment (USA) ${ }^{[9]}$ is a joint collaboration of the Naval Research Laboratory (NRL) and the Stanford Linear Accelerator Center (SLAC) to develop and operate a pointing X-ray observatory. The expected launch date is December 1997. USA is designed to collect large amounts of high resolution X-ray timing data with moderate spectral resolution on 30 of the most luminous sources in the $\mathrm{X}$-ray sky. Each X-ray source will be observed at roughly one microsecond time resolution in the spectral range from 1 to $25 \mathrm{keV}$ for a total collected duration of about one month. The experiment is designed for a required lifetime of three years, with a goal of five years.

As one of several experiments on the Air Force's Advanced Research and Global Observation Satellite (ARGOS), the USA experiment will be mounted on the aft or antivelocity face of ARGOS, shown in Figure 2.6. ARGOS will be launched by a Delta II rocket into a low-earth orbit at an altitude of $834 \mathrm{~km}$ or a nominal orbital period of 102 minutes with an orbital inclination of $98.7^{\circ}$. Onboard gyros will keep the orbit sunsynchronous so that in a period of a year, the orbital plane will sweep the entire celestial sky. 


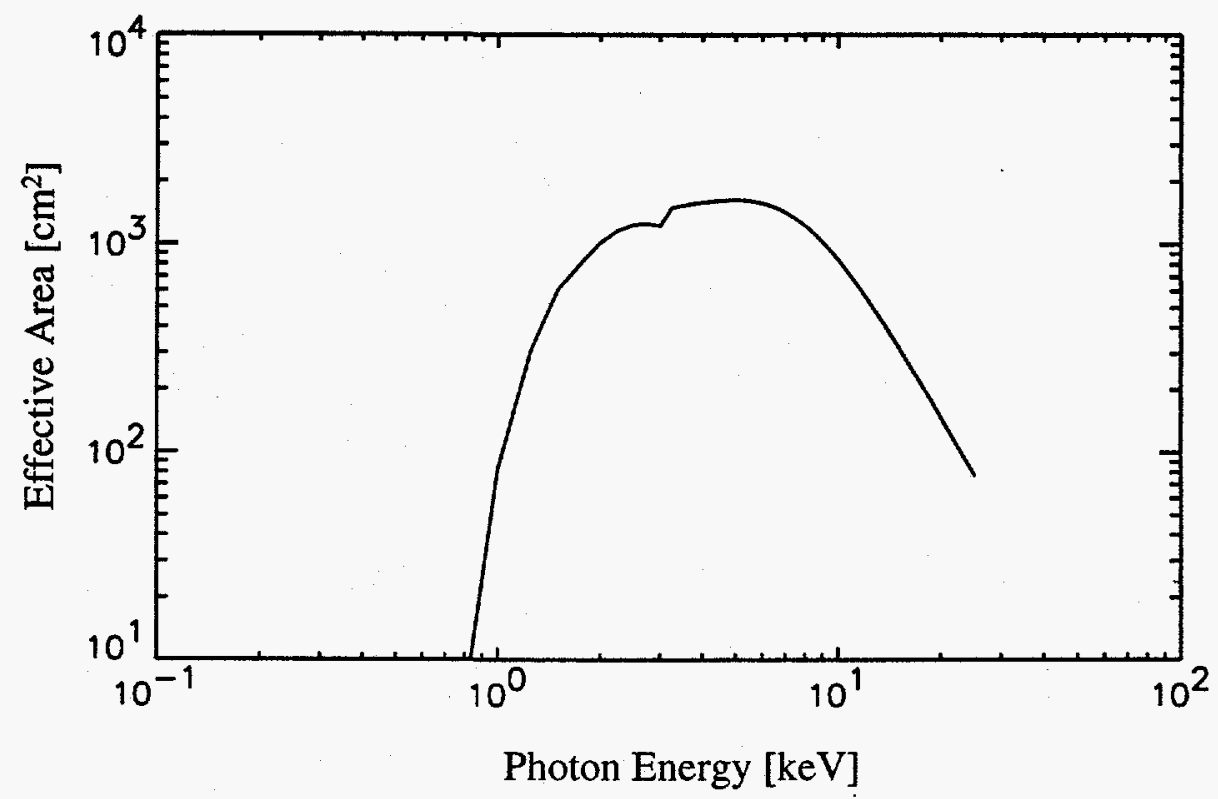

Fig. 2.7 USA effective area for both detector units and all three wire layers as a function of photon energy.

\subsubsection{Instrumentation}

Two independent detector units (DU) with a total collecting area of $2000 \mathrm{~cm}^{2}$ comprise the primary components of the USA experiment. Each detector unit consists of a collimator, a proportional counter, gas system and central electronics. Figure 2.7 shows the effective X-ray acceptance for the USA detectors. The collimator is a honeycomb of hexagonal copper tubes $\left(1.2^{\circ} \mathrm{FWHM}\right)$ formed into eight separate modules to facilitate their construction. Each module is $4 \frac{1}{2}$ " deep with a $3 " \times 11$ " face. They are stacked along the longest dimension in groups of four to form the two segments that fill its support structure, an aluminum collimator frame.

Construction and testing of the USA collimator modules were performed at SLAC. The acceptance of each module to $\mathrm{X}$-rays incident at given relative angles were measured. The X-ray beam consisted of an ${ }^{55} \mathrm{Fe}$ source placed at the end of a long evacuated pipe that was assembled in the PEP Machine tunnel. A detector apparatus was designed and constructed to position the modules and detect the $\mathrm{X}$-rays transmitted through the module. Further details of this testing are given in Appendix D. 


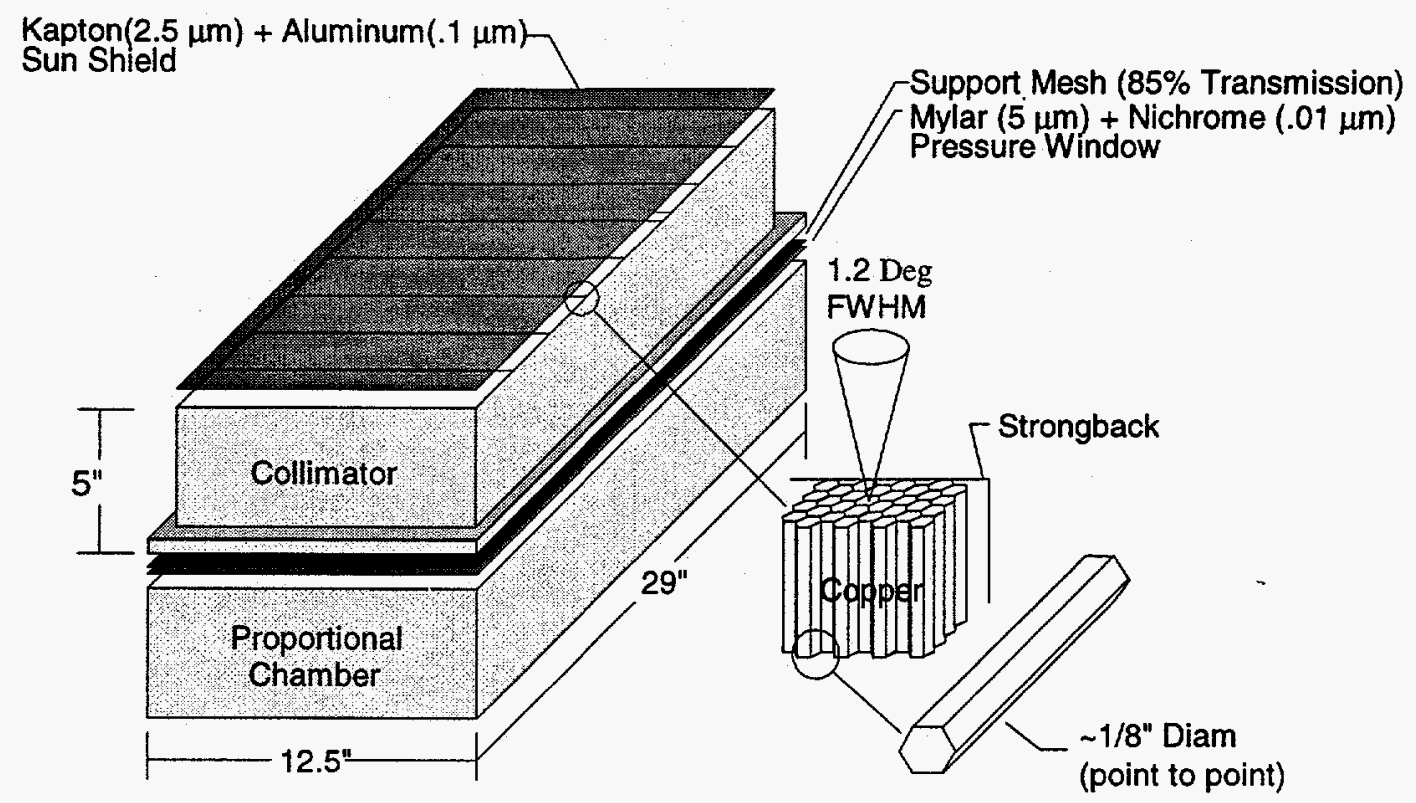

Fig. 2.8 Layout of a USA detector unit with an exploded view of the collimator.

The collimator frame was bolted onto a proportional chamber with a thin window in between to contain the gas volume. The window's thickness was chosen to be $5 \mu \mathrm{m}$ mylar to lower the energy spectrum of detected $\mathrm{X}$-rays to the $1 \mathrm{keV}$ region. A fine wire mesh is placed between the window and the collimator to give the window additional structural support and protection against any small burs on the collimator face. Along the junction of two stacked collimator modules, the two supporting bridge sheets formed a thin separation. At the end of a thermal-vac cycle during preflight testing, the window burst along one of these thin separations, causing a catastrophic collimator failure. Additional details about this incident are provided in Appendix D.7. After this incident, all of the junctions between collimator modules and between the modules and the support frame were taped over to protect against the window expanding into and possibly breaking this junction.

Refurbished from the Spartan -shuttle mission, the multi-wire proportional chamber contains one perimeter veto wire and three wire layers. The chamber is filled with P-10 gas (90\% Argon, 10\% Methane). A spherical titanium gas bottle bolted to the detector backplane supplies the P-10 gas at 2700 psi. The pressure regulation system 
a) USA front view, rotated

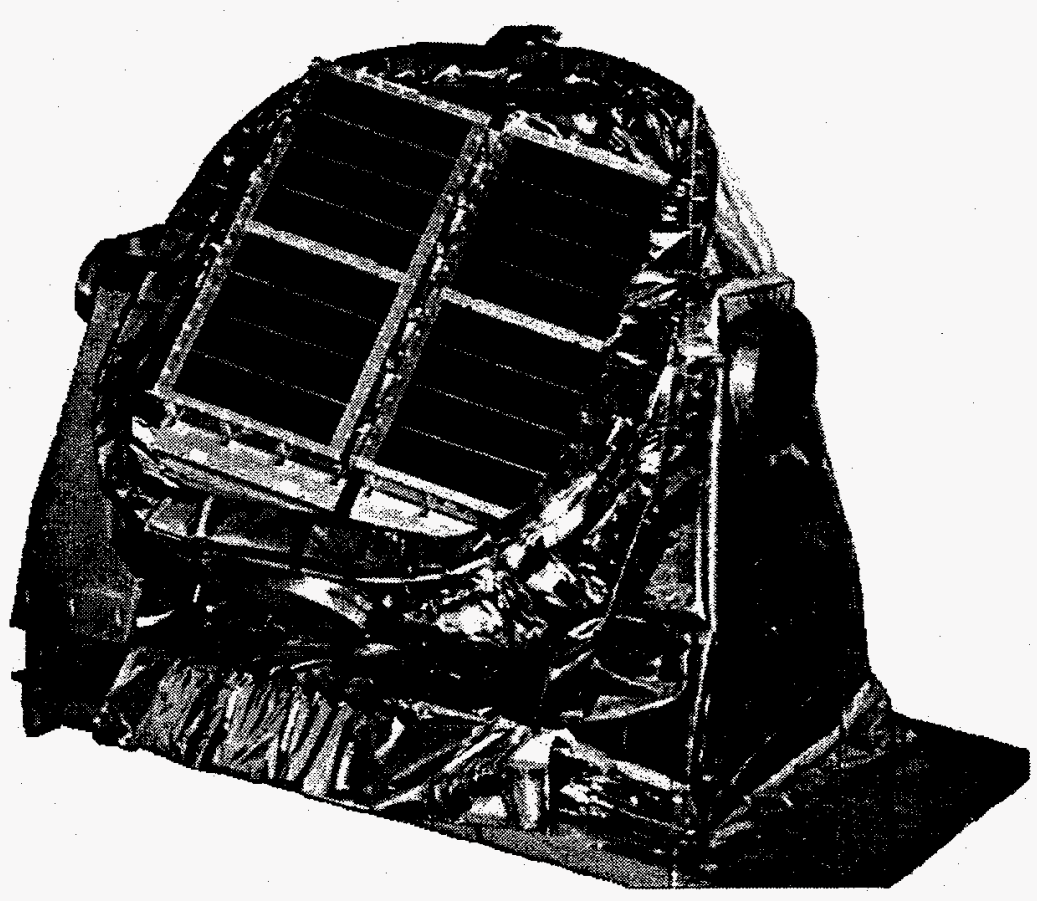

b) USA front view, straight-on

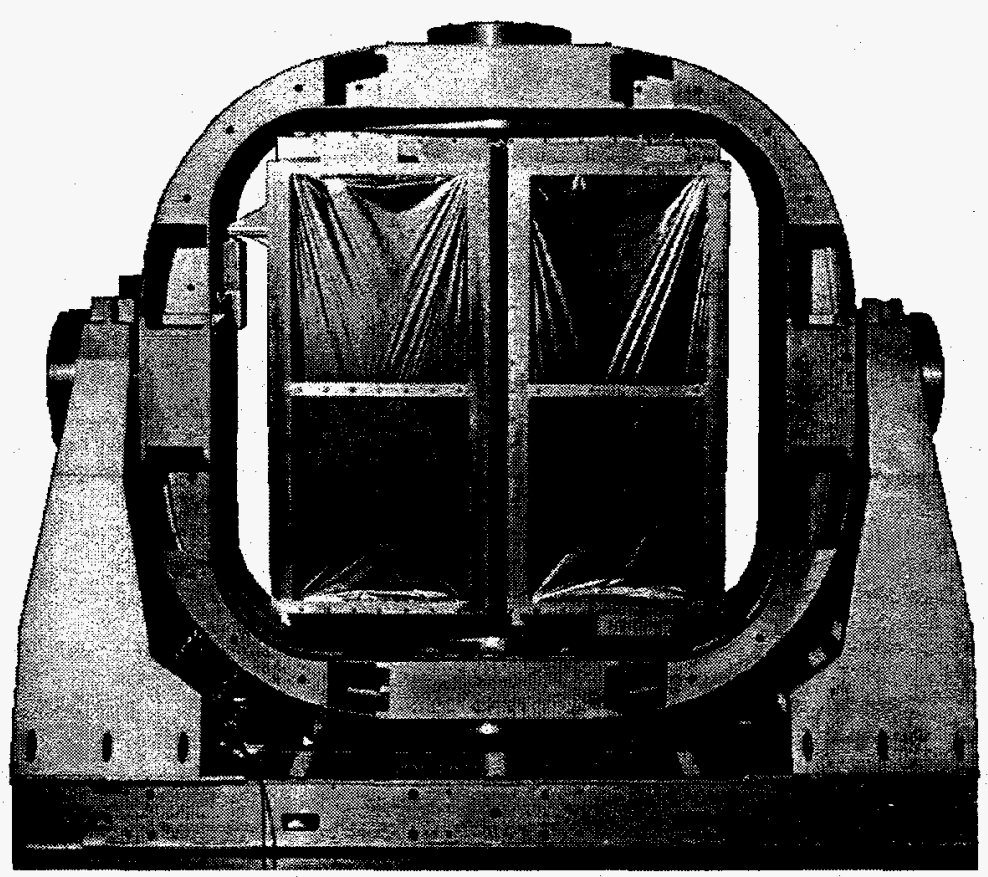

Fig. 2.9 Front views of the Unconventional Stellar Aspect Experiment shown here a) rotated with its collimator face exposed and b) straight-on with its sun shield in place, but without its Multi-Layer Insulation (MLI) thermal blanket. 
a) USA rear view, rotated

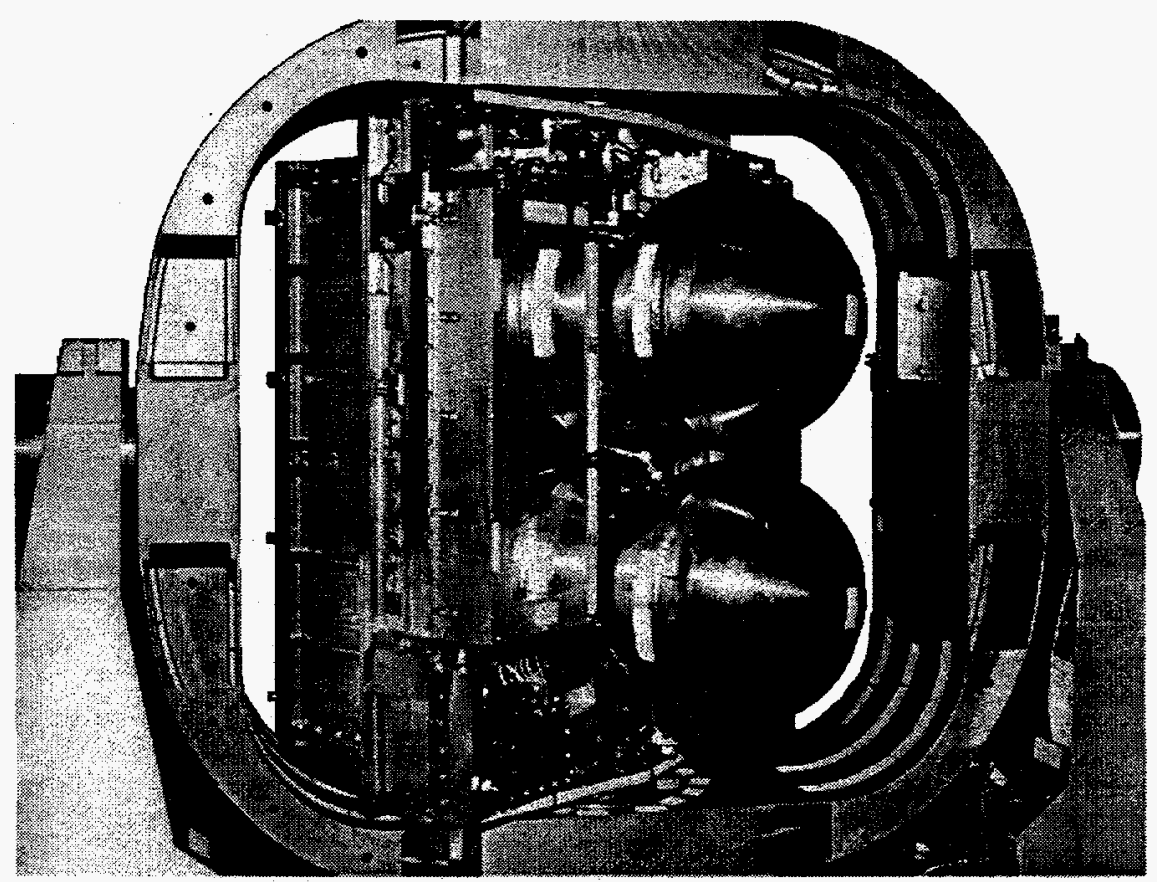

b) USA rear view, straight-on

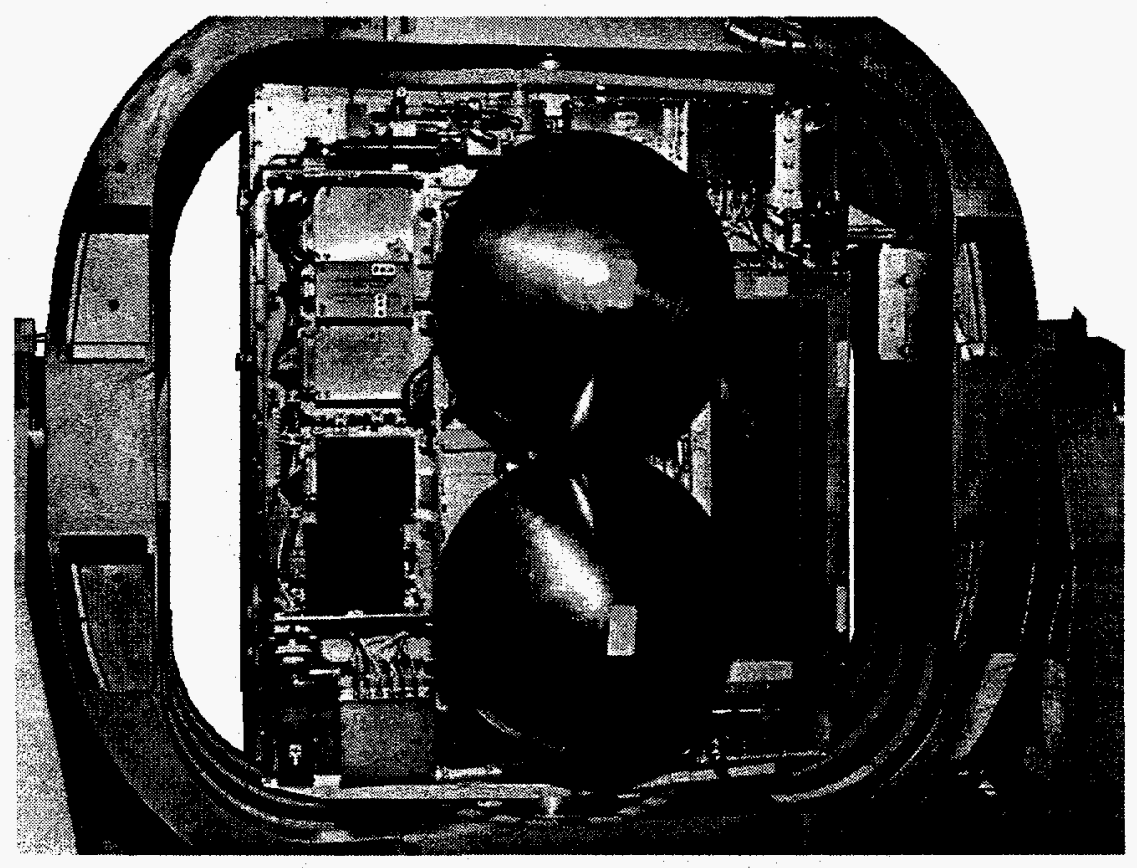

Fig. 2.10 Rear views of the Unconventional Stellar Aspect Experiment shown here without its MLI thermal blanket a) rotated and b) straight-on. The gas tanks are indicated by the two metal spheres, one for each detector unit. The two views are rotated from each other by $180^{\circ}$ about the yoke and the pylon axes. 
maintains the chamber at 14.7 psi.

A $2.5 \mu \mathrm{m}$ layer of aluminized kapton is suspended above the collimator by an aluminum heat shield frame. The layer serves as a heat shield to keep the sun heat input through the face of the collimator to a minimum. Figure 2.8 summarizes the geometry of a USA detector unit.

The two detector units are supported together by a two-axis gimbal system ${ }^{[10]}$. As Figure 2.9 and 2.10 shows, the two detector units are first supported by a yoke along an axis that enables rotations of $\pm 90^{\circ}$ in yaw. The yoke, in turn is supported by two pylons along an axis perpendicular to the first that enables $+70^{\circ} / 100^{\circ}$ rotations in pitch. With this two-axis gimbal system any source within the limits in pitch and yaw as described may be tracked by the USA detector during its orbital motion. 


\section{References}

1) Wood, K. S., et al. (1984), ApJS, 56, 507; Wood, K. S. (1978), "HEAO General Information, 2nd Edition", NRL internal report.

2) Allen, J., Jahoda, K., \& Whitlock, L. (1994), Legacy, 5, 27.

3) Gursky, et al. (1978), ApJ, 223, 973.

4) Gruber \& Whitlock (1994), Legacy, 5, 35.

5) Jahoda, K., et al. (1996), SPIE, 2808, 59.

6) Gruber, D. E., et al. (1996), Astron. Astrophys. Suppl. 120, 641.

7) Lochner, J. C., Remillard, R. A., \& McGlynn, T. A., (1996), Proceedings of the 1996 High Energy Astrophysics Division Meeting in San Diego.

8) Gordon, D. (1992), MIT 64-02006.01.

9) Wood, K. S., et al. (1994), EUV, X-Ray, and Gamma-Ray Instrumentation for Astronomy V, O. H. Siegmund \& J. V. Vallerga, editors, SPIE Proceedings, 2280, 19; Wood, K. S., et al. (1994), The Evolution of X-ray Binaries, S. S. Holt \& C. S. Day, editors, AIP Proceedings, 308, 561.

10) Hanson, J. E., Ph.D. Thesis, "Principles of X-ray Navigation", Aeronautics and Astronautics Department and SLAC, Stanford University, 1996. 
Chapter 3

HEAO A-1 HIGH-BIT-RATE DATA STUDIES

\subsection{Introduction}

The HEAO A-1 High-Bit-Rate (HBR) data is the highest time resolution data available from the A-1 instrument. It was obtained by commanding a special telemetry mode to transmit event times in real time at a one or eight microsecond time resolution. The HBR data were recorded periodically from 1977 to 1979 at time resolutions higher than any other X-ray detector before or after the HEAO-1 mission. HEAO A-1 held this distinction until 1995 when XTE was launched.

Given that distinction, the HBR data represented a unique opportunity to measure the X-ray time variability of astronomical objects at submillisecond timescales. In 1984, Meekins, et. al. ${ }^{[1]}$ analyzed an HBR pointed observation of Cyg X-1. They reported evidence of a cutoff in the time variability at the one millisecond timescale, described further in Section 3.6. Motivated by this finding as evidence for a black hole, the HBR data were recovered in 1995 by a joint effort of the Stanford Linear Accelerator Center (SLAC) and the Naval Research Laboratory (NRL). This Chapter will describe the recovery of the HBR data, the development of a data archive at SLAC, and the subsequent processing and data analysis. 


\begin{tabular}{|l|c|c|l|}
\hline Mode & $\begin{array}{c}\text { Time } \\
\text { Resolution } \\
{[\mu \mathrm{s}]}\end{array}$ & $\begin{array}{c}\text { Maximum } \\
\text { Data Rate } \\
{\left[\times 10^{3} \text { events/sec }\right]}\end{array}$ & Description \\
\hline Bit & 7.8125 & 128 & $\begin{array}{l}\text { Characterized by a change in level of the bit } \\
\text { stream to signify an event. }\end{array}$ \\
\hline Word & 7.8125 & 12.8 & $\begin{array}{l}\text { Characterized by the occurrence of a } 10 \text { bit } \\
\text { sync word to signify an event. }\end{array}$ \\
\hline Frame & 1 & 11 & $\begin{array}{l}\text { Consists of } 11 \mathrm{x} 10 \text { bit words, } 17 \text { bits of sync } \\
\text { and one overflow bit, for a frame length of } \\
128 \text { bits. The } 10 \text { bit data words represent the } \\
\text { time from the beginning of the frame to an } \\
\text { event. This time is in binary counts of the } \\
1.024 \mathrm{MHz} \text { spacecraft clock. }\end{array}$ \\
\hline
\end{tabular}

Table 3.1 Description of the telemetry modes available for the HBR data. Only the Bit mode was used.

\subsection{Description of the HBR Data}

During the normal workhorse mode, the A-1 instrument ${ }^{[2]}$ accumulated X-ray events in sequential 320 or 5 millisecond time bins and stored the resulting data on its onboard tape recorders. The stored normal telemetry data were then downloaded during the spacecraft's next pass over a ground station. For historic reasons, the normal telemetry data are referred to as the Non-Return-to-Zero (NRZ) data. For the HBR data, the onboard tape recorder was not available. The HBR data were instead obtained by preempting the transmission of the normal telemetry data and using the spacecraft's entire $128 \mathrm{kbps}$ data channel to downlink the data in real time. This restricted observations at the HBR time resolutions to 11 minute periods, four times per orbit, when the spacecraft was in direct radio contact with a ground station.

The time resolution of the HBR data varied between one and eight microseconds depending upon which of the three HBR telemetry modes was selected. The majority of the HBR observations were collected at an eight microsecond time resolution in the Bit mode, described in Table 3.1. This is the only mode whose data format is understood. For a detailed description of Bit mode data format see Appendix A.1. 
At the ground stations, the HBR and NRZ data were recorded together onto analog magnetic tapes on separate tracks. The tapes were then forwarded to NRL under an arrangement with NASA, whereby NRL assumed the responsibility for their digitization. At NRL the analog tapes were read with a video tape drive, digitized using a NOVA 800 and the data were written onto 9-track 6250 bpi magnetic tapes.

Except for the Meekins, et. al. analysis in 1984, these magnetic tapes lay primarily in storage at NRL for eleven years. In 1995, a joint effort of SLAC and NRL led to the recovery of these data. This process was made difficult by the paucity of documentation and the dispersion of the personnel familiar with the data, by-products of the extended period of time since the HEAO-1 mission. Nevertheless, with the guidance of K. Wood and D. Yentis, the two resident experts on the HBR data, the magnetic tapes were successfully located and read by a Data General Eclipse. The data were written to disk, one file for each observation. Log book records identified the observations on each tape. The size of the total data sample is 2 gigabytes.

The HBR data were then stored permanently in the SLAC cartridge silo facility onto high density magnetic cartridges. First, the data files were transmitted to SLAC over the internet and written to disk. These files were then transferred and staged to the SLAC staging farm. UNIX shell scripts ${ }^{[3]}$ were written to automate this process. Once in the staging farm, the files were written to one of the cartridges in the SLAC silos.

With the data readily accessible, the next task was understanding the data format. A search of documentation at NRL yielded a few routines that were used in the preliminary data processing and in the Meekins, et. al. analysis. Only one of the routines found described the HBR data format of only one of the telemetry modes, the Bit mode. To process the data with an acceptable performance using the hardware at the time, this routine was written in Data General Assembly. This routine was deciphered after considerable effort with generous assistance from D. McNutt and T. Crandall, and translated into an Interactive Data Language (IDL) ${ }^{[4]}$ routine. The data format of the HBR Bit mode is described in detail in Appendix A. 


\begin{tabular}{|l|l|}
\hline \multicolumn{2}{|c|}{ DATA PROCESSING STEPS } \\
\hline 1) MKHBRECS & Produce "Quick Look" light curves \\
\hline 2) HBRFMT & Re-format raw data into the Photon Time Interval (PTI) format \\
\hline 3) CTIFMT & $\begin{array}{l}\text { Simplify PTI format data into the Concatenated Time Interval } \\
\text { (CTI) format }\end{array}$ \\
\hline
\end{tabular}

Table 3.2 The major data processing steps for creating the HBR data archive.

\subsection{Processing the HBR Data}

The construction of the HBR data archive at SLAC is summarized by the three major production steps shown in Table 3.2. In general, each step reduces the data set size and simplifies the retrieval of event times. All of the production routines were developed at SLAC and the relevant source listings are provided in Appendix A.4. The first step in the data processing examined a quick look of the data. The MKHBRECS routine extracted the light curves from the data. Plots of the light curves revealed that counts near the ends were too high to be interpreted as real count rates, as Figure 3.1 shows. These numbers occur during the periods when either an acquisition is lost or gained over a ground station or when the telemetry mode is switching between HBR and NRZ. The data records corresponding to these regions were cut from further processing.

A product of the real time acquisition of the HBR raw data is a highly inefficient data format. One bit is recorded for each sequential 8 microsecond interval. Namely, information is stored for every time bin. A bit is set if one or more events occur in that 8 microsecond interval or is otherwise cleared. The resulting data is sparsely populated with set bits, even at Crab intensities ( $6 \%)$. The second production step improves the data storage efficiency by storing information for every event instead of every time bin. The HBRFMT routine reformats the HBR raw data into storing the time intervals or waiting times between sequential events. This HBRFMT routine was originally developed at NRL in skeletal form and later rewritten and expanded at SLAC. The format of the reformatted data is called the Photon Time Interval (PTI) format. Further description of the PTI 


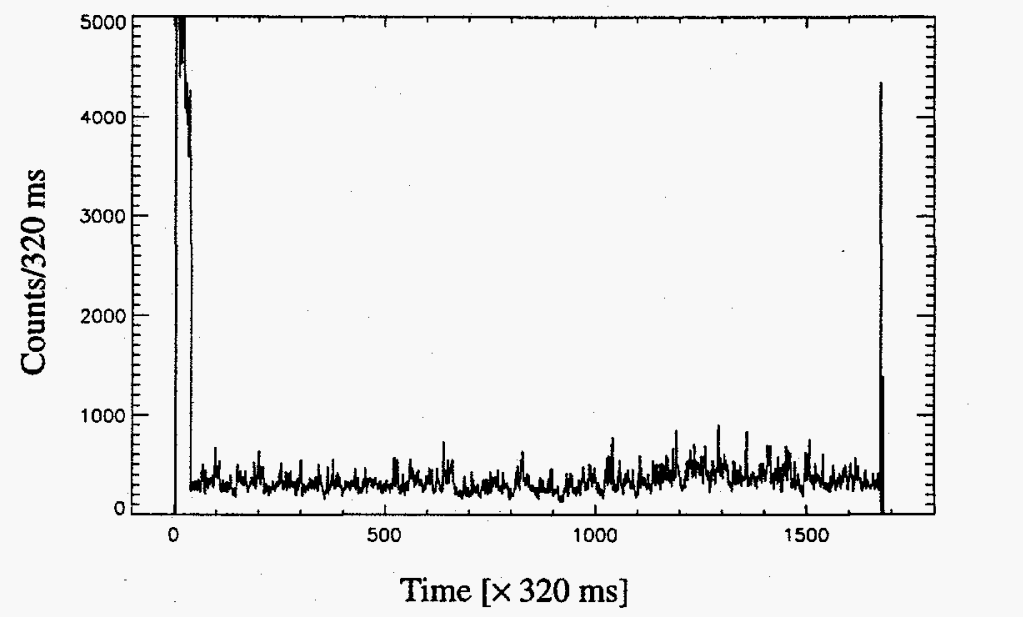

Fig. 3.1 Typical light curve for a HBR raw data file. The ends contain numbers $>$ Crab intensity $\sim 2300$ counts $/ 320 \mathrm{~ms} / 1^{\circ} \times 4^{\circ}$ Module and were cut from further data processing.

format can be found in Appendix A.2. The resulting PTI files are on average 4 times smaller than their raw data predecessors.

Although the PTI format stored photon times efficiently, retrieving those photon times is somewhat cumbersome. The data format was designed for sequentially accessing data in 40.96 second sections. These sections of time, called Major Frames, originate from the format of the normal 320 or 5 millisecond binned NRZ data that delimited sections by Major Frames. The CTIFMT routine concatenates all the Major Frame data segments in the PTI format into arrays, greatly simplifying the retrieval of photon event times. These arrays are stored into a single Interactive Data Language (IDL) structure variable named cat. For a complete description of this IDL structure variable, see Appendix A.3. This IDL structure variable is then saved to an IDL save session file. An IDL save session file is written in a platform independent format called the eXternal Data Representation ${ }^{[5]}$.

Since NASA neither checked nor corrected the HBR data for possible errors, those tasks were left to the data processing at SLAC. Several diagnostic tools were available to help identify errors in the HBR data. Flags associated with downlink errors were included in the HBR data format every 80 microseconds. If any of these error flags was set, then the associated data were cut from further processing. The NRZ data were 
also included in the data stream. Since the NRZ and HBR data collected events over the same period of time, their light curves could be compared and checked for differences. Appendix B describes the procedure for comparing the two light curves in greater detail. Finally, the distribution of waiting times from the HBR data served as another quick look of the data. Anomalous number of occurrences at particular waiting times could be readily identified by examining these waiting time distributions.

Using the diagnostic tools described, a source of noise was identified in the HBR data and was subsequently corrected for. Erroneous waiting times occurred when two adjacent 8 microsecond time bins both recorded an event, called an adjacent pair. However, the detector dead time was 19 microseconds, as discussed later in Section 3.4.1, corresponding to a minimum possible waiting time of two 8 microsecond time bins. These adjacent pairs were found to occur only at a specific location in the raw data format and at a rate that represented only $0.6 \%$ of the total number of events. Comparison of the HBR and NRZ data showed that only one of the two adjacent pairs was present in the NRZ data. Evidence indicates that this noise was a property unique to the HBR data and possibly a product of the digitization process. The HBR data were corrected by randomly removing one of the events in every adjacent pair. This correction was applied during the CTIFMT data processing step and is further described in Appendix B.

\subsection{Instrumental Effects}

In addition to data format errors, instrumental effects can also introduce distortions in the data. Some instrumental effects are unavoidable by-products of detection, such as dead time, while other instrumental effects are due to instrument malfunctions. In the HEAO A-1 detector, both types of instrumental effects were present. We suspect that an electronics malfunction produced systematic distortions in all of the HBR data. The identification and correction of these instrumental effects are necessary

elements for separating real and artificial features in time variability analyses. As discussed later in Section 3.5.2, the instrumental effects in HEAO A-1 introduced distortions in the Meekins, et. al. analysis that completely account for many of the features they attributed to Cyg X-1. 


\begin{tabular}{|c|c|c|}
\hline Dead Time Type & $\begin{array}{c}\text { Observed } \\
\text { Count Rate } \\
R_{\text {obs }} \\
\end{array}$ & $\begin{array}{l}\text { Observed Waiting Time Density } \\
\text { for a Poisson process } \\
f_{o b s}\end{array}$ \\
\hline Non-paralyzable & $\rho /(1+\rho \tau)$ & $U(t-\tau) \rho e^{-p(t-\tau)}$ \\
\hline Paralyzable & $\rho e^{-\rho \tau}$ & $\begin{array}{c}\sum_{j=1}^{K} U(t-j \tau)\left[(-1)^{j-1} /(j-1) !\right] \rho^{j}(t-j \tau)^{j-1} e^{-j \rho} \\
\approx[\rho /(1-\rho \tau)] e^{-\rho t}, \quad t \gg \tau\end{array}$ \\
\hline
\end{tabular}

Table 3.3 Summary of the properties of two types of dead time models. $\rho$ is the true count rate, $\mathrm{t}$ is the waiting time, $K$ is the largest integer smaller than $t / \tau$ and $U$ is the unit-step function.

\subsubsection{Dead Time}

For any detector or electronic circuit there exists a minimum "waiting time" between two events to allow each to be detected. This is referred to as the dead time $\tau$, the length of time that an apparatus is insensitive to additional pulses after the arrival of an accepted event. Two types of dead time are traditionally considered ${ }^{[6]}$, a non-paralyzable dead time and an paralyzable dead time. They differ in how the apparatus responds to the arrival of pulses during a dead time. For a non-paralyzable dead time, the instrument is dead for a fixed amount of time even if another event arrives during the dead time interval. An paralyzable dead time reacts by extending the dead time by $\tau$ from the last pulse arrival time. The properties of these two types of dead time models are summarized in Table 3.3.

For small dead times (i.e. $\rho \tau \ll 1$, where $\rho$ is the true count rate) the distinction between the two types of dead times becomes negligible. To first order in $\rho \tau$, the observed count rate formulae listed in Table 3.3 are identical, $R_{o b s} \approx \rho(1-\rho)$ and the relative error between the two waiting time distributions is $\approx \rho \tau$. For the A-1 instrument, the dead time was 19 microseconds as discussed later in this Section, which gives a $\rho \tau \sim$ $0.06 \ll 1$ at Crab intensities. Thus, the differences between the two types of dead time are negligible in the HBR data analysis and a non-paralyzable dead time is assumed in 


\begin{tabular}{|l|c|c|}
\hline HEAO A-1 Module & 3 & 7 \\
\hline Non-paralyzable Dead Time $[ \pm 0.1 \mu \mathrm{s}]$ & 18.8 & 19.3 \\
\hline
\end{tabular}

Table 3.4 Dead times for Modules 3 and 7 determined from fitting [3.1] to the HBR waiting time distributions.

subsequent analysis for its simpler waiting time distribution.

The observed waiting time distribution is additionally distorted by the binning introduced by the time resolution of the telemetry mode. Namely, the probability of detecting two events within some number of waiting time bins is not simply the integral of the waiting time density over that interval. An event may occur any time within the smallest possible time bin, the length of the time resolution. For the HBR data this time bin is eight microseconds. This information loss must be accounted for in the observed probability distribution. The resulting probability function for a Poisson process

$$
P(n, \rho)= \begin{cases}0, & 0 \leq n \leq \kappa-1, \\ 1-\varepsilon-\left(1-e^{-\mu(1-\varepsilon)}\right) / \mu, & n=\kappa, \\ \varepsilon+\left(1+e^{-\mu(1-\varepsilon)}\left(e^{-\mu}-2\right)\right) / \mu, & n=\kappa+1, \\ \left(\frac{\sinh (\mu / 2)}{\mu / 2}\right)^{2} \mu e^{-\rho(n T-\tau)}, & n>\kappa+1\end{cases}
$$

is derived in Appendix C.1, where $\rho$ is the count rate of the Poisson process; $n$ is number of waiting time bins; $\kappa$ is the largest integer $\leq \tau / T ; \varepsilon$ is the residual $\tau / T-\kappa$; $T$ is the time resolution; $\tau$ is the dead time; and $\mu$ is the mean number of counts per bin, $\rho T$.

The dead times for HEAO A-1 Modules 3 and 7 were determined by fitting [3.1] to the HBR waiting time distribution at small waiting times $(<1$ millisecond). The method of non-linear least squares was used. A summary of the fit results is shown in Table 3.4. The dead time is bounded below by the smallest waiting time recorded, two time bins. The dead time is bounded above by three time bins, otherwise three time bins 
would be the smallest waiting time recorded. These lower and upper limits of two and three time bins correspond to dead times, 15.6 and 23.4 microseconds, respectively.

\subsubsection{HEAO A-1 Module Electronics Malfunction}

A reset problem involving coincidence events was known to exist in the HEAO A-1 module electronics. The $\mathrm{X}$-ray energy spectrum was contaminated by the charge particle spectrum because the charge held in the electronics circuitry was not properly reset when a coincidence event (i.e. charged particle) occurred. The pulse amplitude recorded for the next X-ray event would be the larger of the current X-ray event or the held pulse height of the charged particle.

An exhaustive inspection of the HBR data has revealed another systematic distortion, this time of the Fourier power spectrum. A strong suspect for this previously undetected problem was modelled as a malfunction of the HEAO A-1 module electronics. The distortion resulting from modelling this suspected detector malfunction for a Poisson source is shown in Figure 3.2. The noise floor at lower frequencies is raised above the floor at higher frequencies. A broad "knee" from 100 to 1000 Hertz joins the two levels. These features sharply contrast to the flat spectrum expected for a Poisson source. The waiting time distribution for a Poisson source also showed a systematic distortion from its expected form. As Figure 3.2 shows, a smooth "kink" is introduced into its exponential at approximately one millisecond.

Neither of these distortion features could have been detected in the well-studied NRZ data. This may explain why this problem remained undetected. The highest time resolution for the NRZ data is 5 milliseconds, corresponding to a Nyquist frequency of $100 \mathrm{~Hz}$. This is just where the "knee" distortion starts in the Fourier power spectrum. The NRZ data were binned. Therefore, waiting time distributions that require individual photon times could not be obtained.

Similar distortions were discovered by my colleague at SLAC, G. Shabad, in preflight USA calibration data. After extensive modelling and examination of the USA electronics, G. Shabad and G. Godfrey of SLAC determined that the cause of the USA 


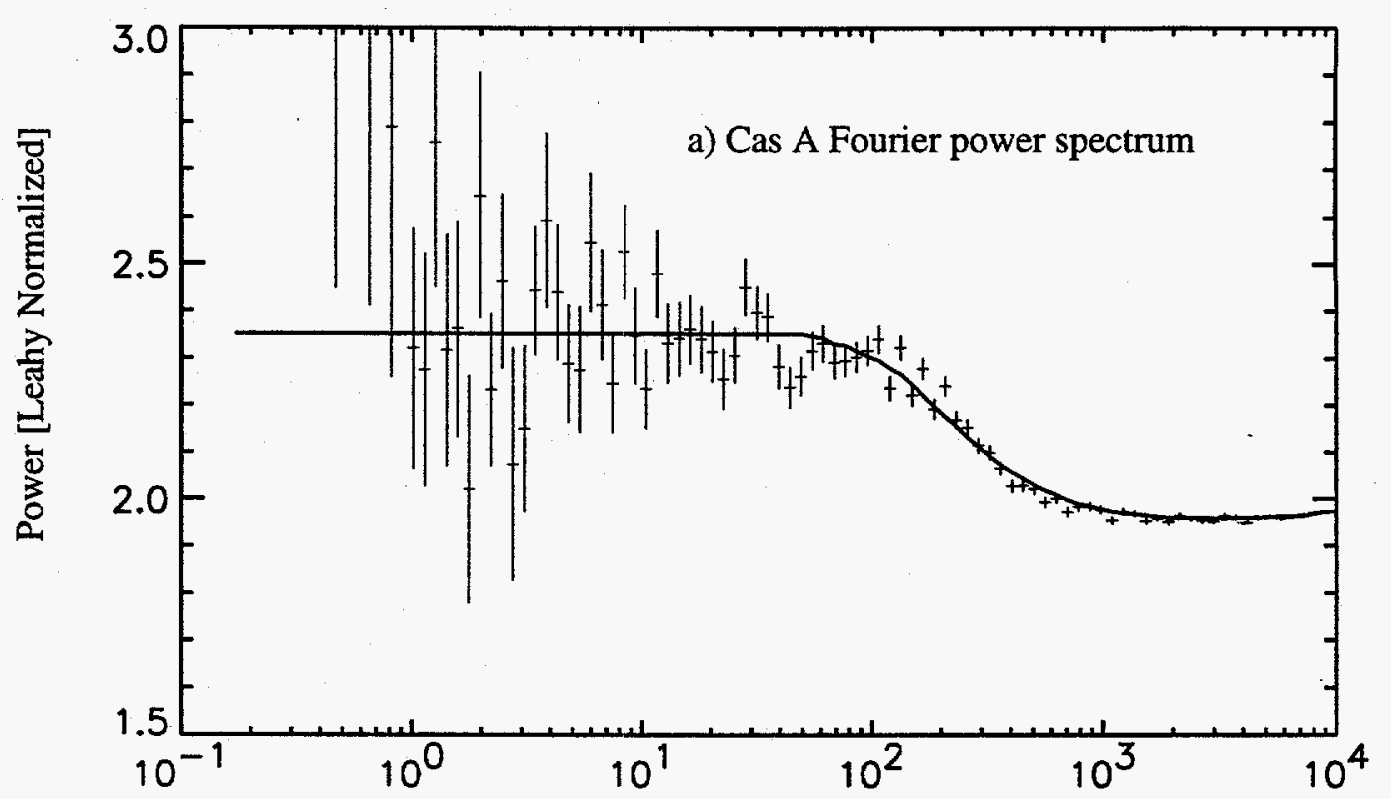

Frequency $[\mathrm{Hz}]$

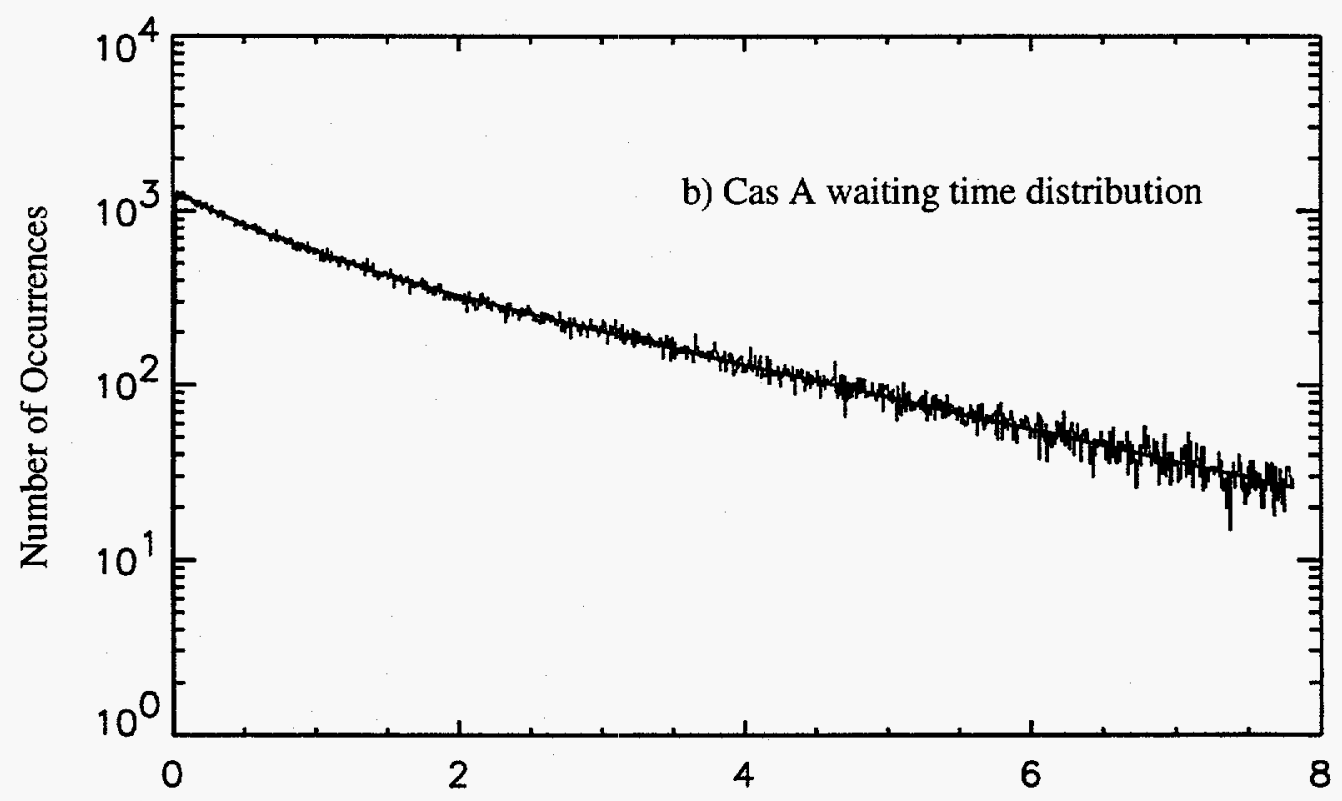

Waiting Time [ms]

Fig. 3.2 The a) Fourier power spectrum and the b) waiting time distribution of a Poisson source, Cas A. Distortions from the expected Poisson characteristics are seen by the bi-level spectrum and a "kink" in the exponential. The solid curves in both plots are the result of fitting an offset hyperexponential distribution to the waiting time distribution shown. Goodness of fit is: $\chi^{2} /$ dof $=115 / 85$ for the Fourier spectrum and $\chi^{2} /$ dof $=1061 / 993$ for the waiting time distribution. 
distortions was a faulty reset of the perimeter veto electronics. Normally, when a charged particle traverses the USA detector, it generates a coincidence between the perimeter veto wire and one or more chamber wires. For such events, the perimeter veto is reset properly. However, for some charge particle trajectories only the perimeter veto wire fires. For such events, the perimeter veto fails to reset. The only way it can be cleared is if another perimeter veto event occurs or if a chamber wire fires. If the next event is an incident X-ray, it will be vetoed regardless of when it arrives. This amounts to vetoing asynchronous coincidences between charged particle and X-ray events. This faulty perimeter veto logic introduces an effective variable dead time. The resulting waiting time density, $f_{p v m}$ for the perimeter veto model (PVM) is given by

$$
\begin{gathered}
f_{v x}(T)=\left(\begin{array}{c}
v-1 \\
x-1
\end{array}\right) \frac{\left(\rho_{v} T\right)^{v}\left(\rho_{\gamma} T\right)^{x}}{(v+x) !} \rho_{\gamma} e^{-\left(\rho_{v}+\rho_{\gamma}\right) T} \\
f_{p v m}(T)=\sum_{v=1}^{\infty} \sum_{x=1}^{v} f_{v x}(T)
\end{gathered}
$$

where $T$ is the waiting time, $\rho_{\gamma}$ is the $\mathrm{X}$-ray source rate and $\rho_{v}$ is the perimeter veto rate. The $f_{v x}(T)$ term is the probability density for a waiting time $T$ between two photons if $v$ perimeter veto events and $x$ photons arrive within that interval of time. Appendix C. 2 gives a detailed derivation of Equation [3.2]. Fortunately, the USA electronics have since been modified before launch to correct this perimeter veto reset problem.

If we assume that a problem similar to that discovered in preflight USA electronics existed in the HEAO A-1 module electronics, then [3.2] provides a reasonable model for fitting the data. However, this scenario could not be confirmed nor denied for HEAO A-1 due to the inaccessibility and lack of archival records of the electronics diagrams. In addition, perimeter veto rates were not recorded in the HBR nor in the NRZ data.

The distortions in the data can be approximated with sufficient accuracy by using an offset hyperexponential distribution ${ }^{[7]}$, 


$$
f_{H}(t)=U(t-\tau)\left(p_{1} \rho_{1} e^{-\rho_{1}(t-\tau)}+\left(1-p_{1}\right) \rho_{2} e^{-\rho_{2}(t-\tau)}\right)
$$

where $\tau$ is the non-paralyzable dead time, $\rho_{1}$ and $\rho_{2}$ are the count rates from two Poisson processes and $p_{1}$ is the probability of generating a waiting time from the first process. The convention chosen here will be that $\rho_{1}>\rho_{2}$. Within the context of the perimeter veto model, the $\rho_{1}$ and $\rho_{2}$ parameters may be interpreted as "effective" Poisson rates.

Equation [3.3] is the linear combination of the waiting time densities for two Poisson processes with the same non-paralyzable dead time The resulting probability function for this offset hyperexponential process is

$$
P_{H}(n)=p_{1} P\left(n, \rho_{1}\right)+\left(1-p_{1}\right) P\left(n, \rho_{2}\right)
$$

where the $P(\mathrm{n}, \rho)$ are given by [3.1]. The solid curves in Figure 3.2 show the result of a non-linear least squares fit of [3.4] to the Cas A data set.

\subsection{Data Analysis}

A challenge for any time variability analysis is to differentiate real features from instrumental effects. For the HEAO A-1 HBR data, this is underscored by significant distortions to the Fourier power spectrum, characterized by a "knee" as previously described. Two types of time variability analyses will be explored with the Cyg X-1 data: the Meekins, et. al. analysis and the Fourier spectra technique. As later discussed, instrumental effects play a significant role in both analyses.

\subsubsection{Correcting the Poisson Noise Floor}

The Poisson noise floor is defined as the Fourier power spectrum of a Poisson process. In the absence of any instrumental effects, the Poisson noise floor is frequency independent with a value of 2 using the Leahy normalization ${ }^{[8]}$. In the HEAO A-1 instrument, the detector dead time and the suspected HEAO A-1 module electronics malfunction each introduced a distortion to this Poisson floor. The dead time lowers the Poisson floor by a small and approximately constant value $(\sim 2 \%)$ over the frequencies of 


\begin{tabular}{|c|c|c|c|c|}
\hline HBR Data File & $\tau[\mu \mathrm{s}]$ & $\rho_{1}[\mathrm{~Hz}]$ & $\rho_{2}[\mathrm{kHz}]$ & $\mathrm{p}_{1}$ \\
\hline $086 \_7 \_7$ & 18.8 & 935 & 2.05 & 0.53 \\
\hline $015 \_2 \_3$ & 18.8 & 991 & 2.18 & 0.52 \\
\hline
\end{tabular}

Table 3.5 The offset hyperexponential distribution parameters that defined the effective Poisson noise floor for two Cyg X-1 data files.

interest $(0.1 \mathrm{~Hz}$ to $4 \mathrm{kHz})$. The suspected electronics malfunction introduces a strong frequency dependent distortion of the Poisson noise floor. As Figure 3.2 shows, the noise floor is distorted into a bi-level spectrum with a broad knee from 100 to $1000 \mathrm{Hertz}$ that joins the two levels. The frequency width and position of this knee vary from source to source.

While the distortions to a Poisson source are readily modelled by fitting [3.4] to the waiting time distribution, the same procedure does not necessarily apply for a nonPoisson source such as Cyg X-1. Excluding any instrument effects, the waiting time distribution for a non-Poisson source is a non-exponential. The effects on nonexponential distributions due to the suspected electronics malfunction are not well understood. However, the Fourier spectra for non-Poisson sources are observed to also have knees characteristic of Poisson sources. The beginning of the knee region is defined as the frequency where the power spectrum has an inflection. For the Cyg X-1 data, this inflection point occurs at $40 \mathrm{~Hz}$. To determine the effective Poisson noise floor for a nonPoisson source, the offset hyperexponential distribution, [3.4] is used to fit to the data's power spectrum from the beginning of the knee region to the Nyquist frequency.

The procedure for determining the effective Poisson noise floor for Cyg X-1 consists of first fitting [3.4] to the waiting time distribution. Random waiting times are drawn from [3.4] as defined by the fitted parameters and accumulated into absolute times. The Fourier spectrum of the simulated event times is calculated in the same manner as the data. Finally, the chi-squared of the fit to the data is calculated at frequencies above 100 Hertz. This procedure is repeated for a grid of parameter values whose origin is defined by the initial fit of [3.4] to the data's waiting time distribution. Table 3.5 summarizes the 
set of parameters that minimized the chi-squared for the Cyg $\mathrm{X}-1$ data. The resulting Fourier power spectrum from these parameters defined the effective Poisson noise floor.

\subsubsection{Correcting the Meekins, et. al. Analysis}

Meekins, et. al. ${ }^{[1]}$ attempted to quantify the aperiodic variability of Cyg $X-1$ by defining a new statistical quantity called the relative integral power. This new statistic was defined by Meekins, et. al. as the total discrete Fourier transform power of the mean subtracted time series divided by the square of total number of counts, $N^{2}$ in the time series,

$$
P_{\text {rel }} \equiv \frac{\sum_{j=-m / 2}^{j=m / 2-1}\left|a_{j}\right|^{2}-a_{0}^{2}}{N^{2}}=\frac{\chi^{2}}{N}
$$

where $a_{j}$ are the standard Fourier coefficients defined in Section 1.2 and $m$ is the number of bins in the time series. The chi-squared, $\chi^{2}$, here is constrained by the sample mean, $N / m$, substituted for both the expected value and the variance of the counts. The timescale associated with [3.5] is defined by the range of Fourier frequencies allowed, namely the Nyquist and the smallest non-zero frequency.

To improve the signal-to-noise, the relative integral power had to be averaged over a large number of independent segments of data. A given time series is first divided into equal length segments. Each segment contained $m$ bins. The relative integral power was calculated for each segment and then the resulting quantities were averaged.

The relative integral power defined by [3.5] contains contributions from both intrinsic variability and extrinsic noise. Meekins, et. al. separated out these two contributions in a formal derivation that showed that

$$
\left\langle P_{\text {rel }}\right\rangle=\left[\left\langle\chi^{2}\right\rangle-\left\langle\chi^{2}\right\rangle_{\text {noise }}\right] /(\langle N\rangle-1)
$$

where $\left\langle P_{\text {rel }}\right\rangle$ is the average relative power due to intrinsic variability, and $\left\langle\chi^{2}\right\rangle$ and $\left\langle\chi^{2}\right\rangle_{\text {noise }}$ are the average chi-squareds of the data and the expected extrinsic noise, 


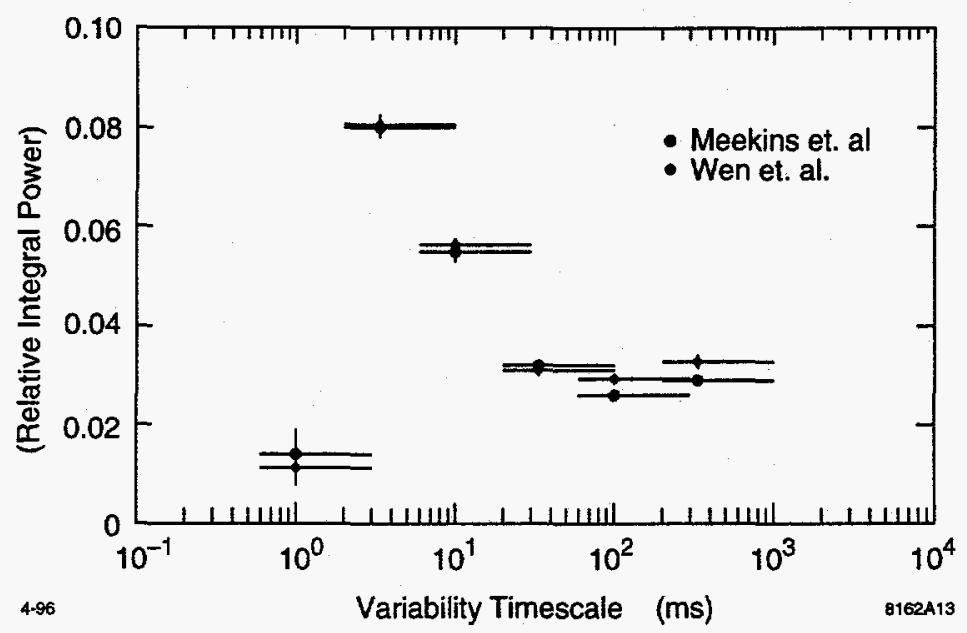

Fig. 3.3 The average relative integral power for Cygnus X-1 from Meekins, et. al. and a reanalysis by Wen, et. al. using the general theoretical framework of Meekins, et. al.

respectfully. The same constraints defined for $\chi^{2}$ in [3.5] also applied to these two averaged quantities. $\langle N\rangle$ is the average total number of counts in one data segment of $m$ bins. This average relative integral power intrinsic to a source will be subsequently referred to as the relative power for brevity.

Equation [3.6] was applied to nine minutes of HBR data on Cyg X-1 observed by HEAO A-1 on May 7, 1978, while Cyg X-1 was in its low state, (observation 086_7_7 listed in Table B.2). The resulting relative power is shown in Figure 3.3. Cyg X-1 exhibits excess power between the 1 - 10 millisecond timescales that cutoff at the 1 millisecond timescale. Meekins, et. al. attributed these features to activity near the inner edge of an accretion disk. In 1996, the Meekins, et. al. result was reproduced using the Meekins, et. al. theoretical framework ${ }^{1}$ with good agreement ${ }^{2}$ as shown in the plot in Figure 3.3.

\footnotetext{
${ }^{1} \mathrm{~A}$ value of 9 for $\chi^{2}$ for sets with no counts was chosen instead of 0 as reported by Meekins, et. al. because using a value of 0 could not reproduce the relative integral power at the $0.6-3 \mathrm{~ms}$ timescale.

${ }^{2}$ Remaining discrepancies may be attributed to differences in bin offset and analyzing data from a separate digitization of the original analog tape from Meekins, et. al.
} 
The impact of possible instrumental effects on this result was not quantitatively addressed. Meekins, et. al. neglected the effects of dead time in their analysis and additionally, did not detect the suspected HEAO A-1 module electronics malfunction. To allow a more straightforward correction of dead time and other instrumental effects, the definition of the relative power is first re-examined.

As in [3.5], we define $\chi^{2}$ in terms of the sum of all the Leahy normalized Fourier powers, $P_{\mathrm{j}}$

$$
\begin{aligned}
& \chi^{2}=\sum_{j=1}^{m / 2-1} P_{j}+\frac{1}{2} P_{m / 2} \\
& P_{j}=2\left|a_{j}\right|^{2} / N
\end{aligned}
$$

where $m$ is the total number of time bins in each data segment, (chosen as 10 for the Meekins, et. al. analysis). The average of the chi-squared in [3.7] over the entire ensemble of $m$ bin data segments can be approximated by,

$$
\left\langle\chi^{2}\right\rangle \approx\left(\frac{m-1}{2}\right)\langle P\rangle
$$

where $\langle P\rangle$ is the average Leahy normalized power over the entire ensemble of $m$ bin data and the set of frequencies $\left(f_{j}=1 / T, 2 / T, . . m / 2 T\right) . T$ is the length of an $m$ bin data segment. Using [3.8] to substitute for the average chi-squared in [3.6] yields,

$$
\left\langle P_{\text {rel }}\right\rangle \approx\left(\frac{m-1}{2}\right)\left[\langle P\rangle-\langle P\rangle_{\text {noise }}\right] /(\langle N\rangle-1)
$$

The average chi-squared of the expected intrinsic noise is simply proportional to the usual Poisson noise floor. By ignoring any instrumental effects, the Meekins, et. al. analysis effectively chose a value of 2 for the expected noise floor. The average Fourier power, $\langle P\rangle$ in [3.9], is approximated by the Fourier power described in Section 1.2 at the logarithmic frequency interval that spans the set of frequencies $\left(f_{j}, j=1 . . m / 2\right)$. This approximation ignores possible contamination of variability from other timescales. The 


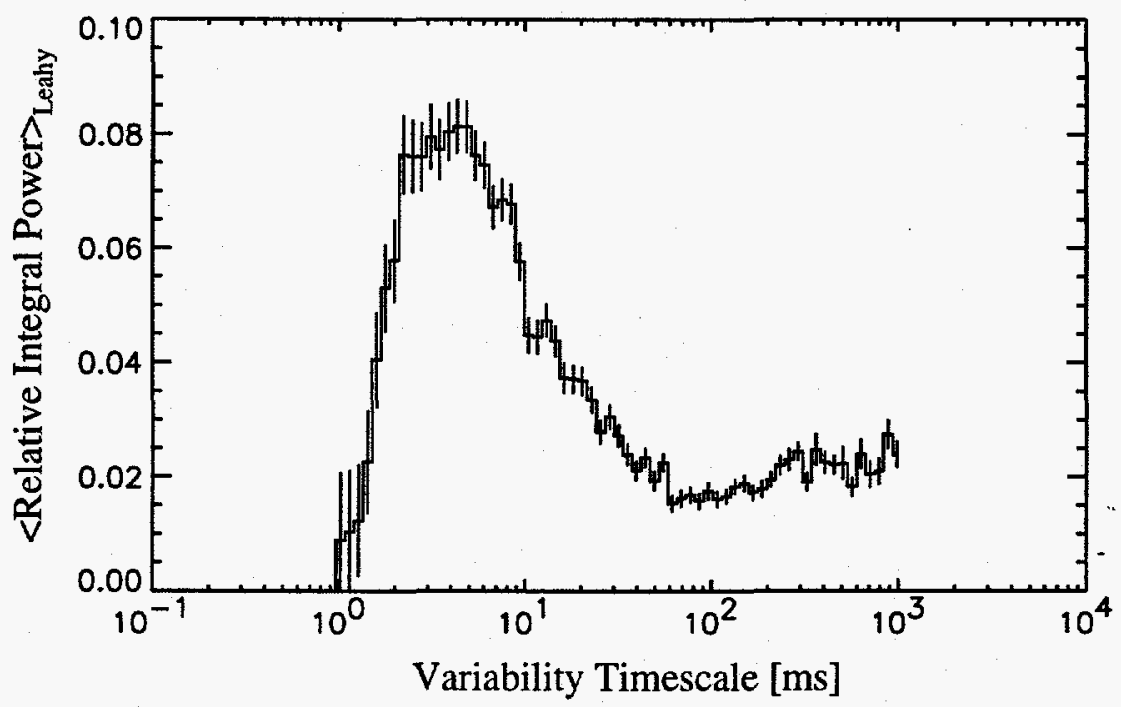

Fig. 3.4 The average relative integral power for Cyg X-1 approximated by using Leahy normalized Fourier power. As in the Meekins, et. al. analysis, dead time and instrumental effects were ignored here.

result of applying [3.9] to the data and ignoring instrumental effects is shown in Figure 3.4. Good agreement is seen between Figures 3.3 and 3.4 using the two methods for calculating the relative power, [3.6] and [3.9]. Any contamination of variability from other timescales is negligible in this data compared to the sharply peaked feature in the relative power from the one to ten millisecond timescale.

By using [3.9] to approximate the relative power, the procedure for correcting instrumental effects in the HEAO A-1 data is straightforward. First, the Poisson noise floor is calculated with the instrumental effects taken into account by applying the technique discussed in Section 3.5.1. The average Fourier power over the $m$ bins of a given timescale is again approximated by the usual Fourier power spectrum as previously described.

\subsection{Results}

The relative power distribution of Figure 3.4 can now be corrected for instrumental effects. Figure 3.5 shows the relative power after correcting the Poisson noise floor for dead time and then additionally for the suspected HEAO A-1 module 

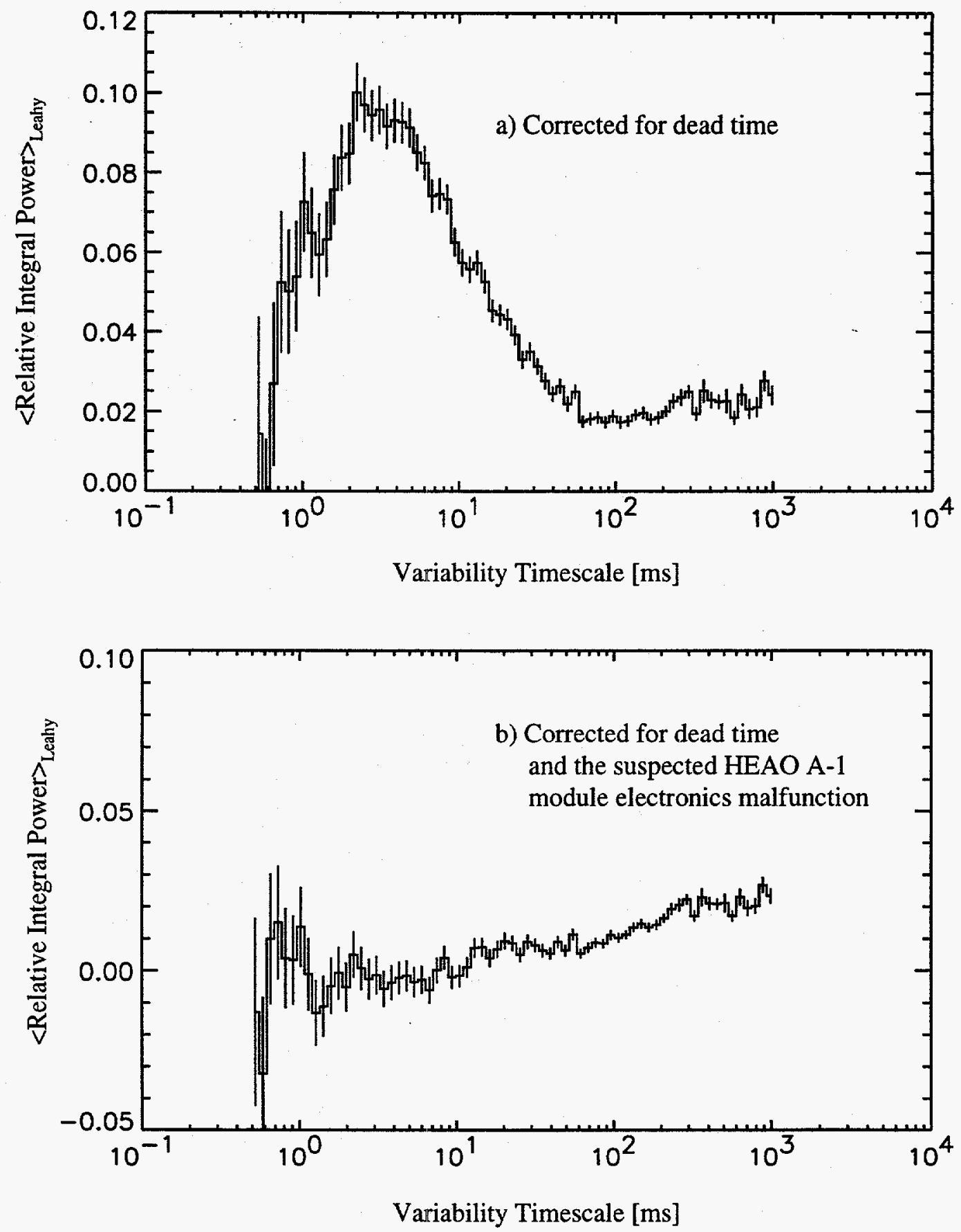

Fig. 3.5 The average relative integral power for Cyg X-1 after the Poisson noise floor is corrected for a) dead time and b) dead time and the suspected HEAO A-1 module electronics problem. The Fourier power are used to approximate the average relative integral power, as described in Section 3.5.2. 
electronics malfunction. The peak in relative power is actually broadened and enhanced after correcting the Poisson noise floor only for dead time. This is due to the slight lowering of the Poisson floor in the presence of dead time. However, this larger peak in relative power is eliminated once the corrections due to the suspected electronics malfunction have been additionally applied to the Poisson noise floor. This analysis indicates that the excess relative power between the $1-10$ millisecond timescales that was previously reported by Meekins, et. al. can be attributed to previously undetected instrumental effects.

Using the approximation [3.9], the statistic that defines the relative power can be reinterpreted from the perspective of Fourier power. The numerator of [3.9] is the total noise-subtracted Fourier power. The denominator in [3.9] scales linearly with timescale since the width of each time bin is proportional to the timescale. The relative power is proportional to the total intrinsic Fourier power per timescale. Figure $3.5 \mathrm{~b}$ shows that this total power per timescale steadily decreases with smaller timescales.

The HEAO-1 spacecraft made a total of two pointed observations of Cyg X-1 using the high-bit-rate telemetry mode. Meekins, et. al. analyzed the first of these observations. The second observation occurred on November 9, 1978, (observation 015_2_3 listed in Table B.2), while Cyg X-1 was again in its low state based on its mean count rate. Both observations each lasted 8.5 minutes and were made while Cyg X-1 was in its low state based on its average count rate of $1071 \mathrm{cts} \mathrm{s}^{-1}$.

The Fourier power spectra technique was applied to both of these pointed observations. For each observation, the Poisson noise floor was corrected for instrumental effects as described in Section 3.5.1. To further improve the statistics, the Fourier power spectra from the two observations were averaged together. Figure 3.6a shows the resulting power spectra of Cyg X-1 and the simulated Poisson noise floor corrected for instrumental effects. Their difference is shown in Figures $3.6 \mathrm{~b}$ and $3.6 \mathrm{c}$.

To measure the significance of the noise-subtracted power, the $95 \%$ confidence level for detecting a signal above the Poisson noise floor is overlayed in Figures 3.6b and 

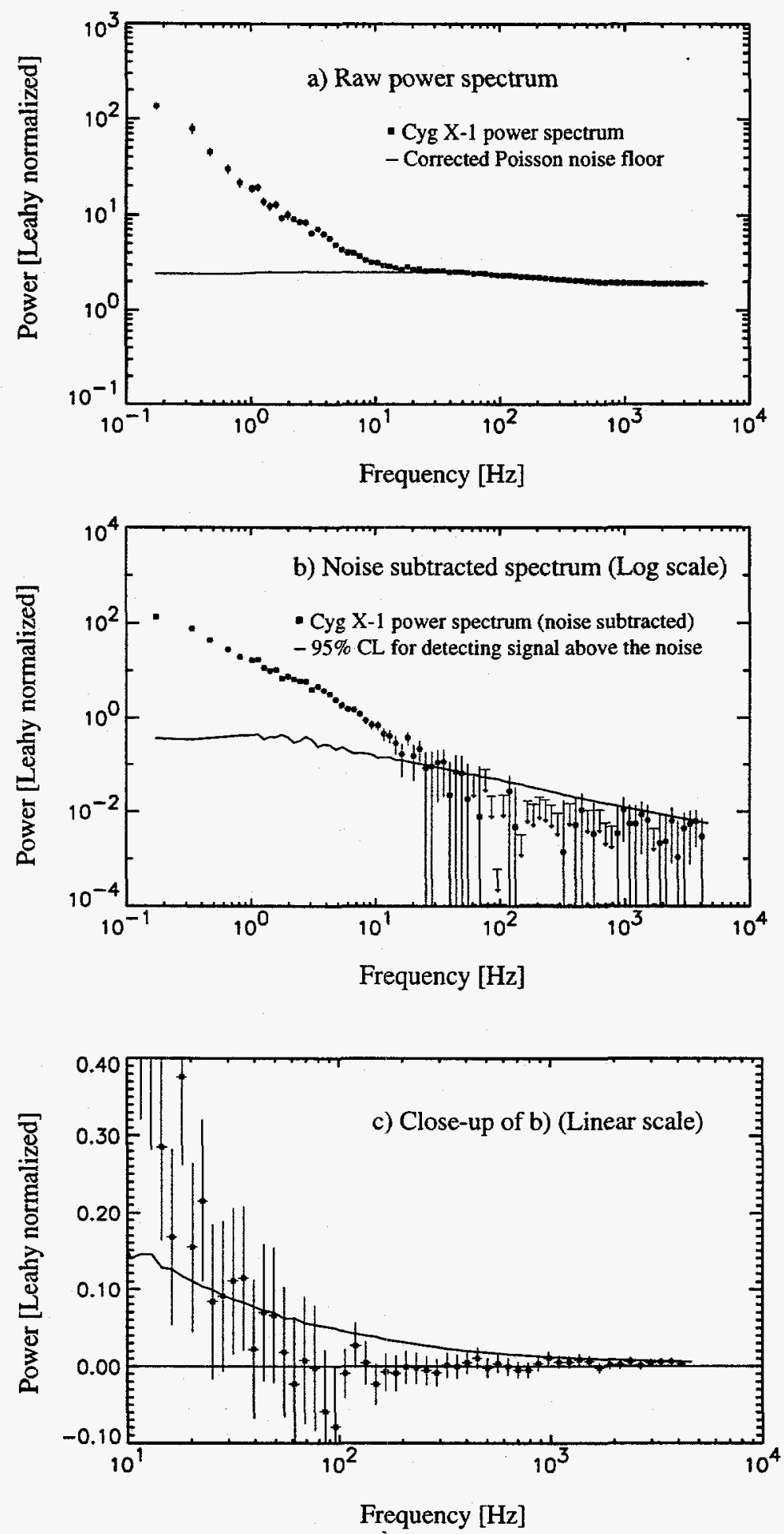

Fig. 3.6 The a) raw and noise-subtracted Fourier power spectrum of Cyg X-1 from the HBR data in b) full and c) close-up views. The solid curve represents the $95 \%$ confidence level for detecting a signal above the Poisson noise floor. Excess power at frequencies greater than $20 \mathrm{~Hz}$ (shaded region) may be contaminated by residual instrumental effects. 


\begin{tabular}{|l|c|c|c|c|c|c|}
\cline { 2 - 7 } \multicolumn{1}{c|}{} & \multicolumn{2}{|c|}{ Power Law Before Apparent Break } & \multicolumn{3}{|c|}{ Power Law After Apparent Break } \\
\hline Detector & $\begin{array}{c}\text { Frequency } \\
\text { Range } \\
{[\mathrm{Hz}]}\end{array}$ & $\begin{array}{c}\text { Photon } \\
\text { Index }\end{array}$ & $\chi^{2} /$ dof & $\begin{array}{c}\text { Frequency } \\
\text { Range } \\
{[\mathrm{Hz}]}\end{array}$ & $\begin{array}{c}\text { Photon } \\
\text { Index }\end{array}$ & $\chi^{2} /$ dof \\
\hline HEAO A-1 & $0.1-3$ & $1.20 \pm 0.08$ & $15 / 13$ & $3-40$ & $1.7 \pm 0.2$ & $18 / 19$ \\
\hline
\end{tabular}

Table 3.6 Summary of power law fits to the noise-subtracted Fourier power spectrum. The break between the two different power laws occurs at $3 \mathrm{~Hz}$.

3.6c. The overlayed curve is 1.96 times the standard deviations of the corrected Poisson noise floor based on the same total number of counts as the data. Excess power is observed at frequencies less than $40 \mathrm{~Hz}$ with greater than a $95 \%$ confidence level. At greater frequencies the noise-subtracted spectrum is consistent with the null hypothesis as expected. The Poisson noise floor was corrected for HEAO A-1's instrumental effects by fitting to the power spectrum of Cyg X-1 for frequencies greater than $40 \mathrm{~Hz}$.

Between frequencies 0.1 and $20 \mathrm{~Hz}$, the spectrum follows power laws with a break to a steeper photon index at frequencies greater than $3 \mathrm{~Hz}$. Power laws were fit separately to the frequency region before and after the break, and the results are presented in Table 3.6. The power spectrum follows a $\sim 1 / f$ spectrum before the break and then falls more steeply afterwards.

No evidence was seen in the HBR data on Cyg X-1 for QPOs. A 95\% confidence level upper limit was calculated for detecting QPOs of a given fractional root-meansquare (rms) amplitude over a given logarithmic frequency interval. The fractional rms normalization is the Leahy normalization divided by the mean X-ray intensity, and can be thought of as a mean amplitude of the intensity variations expressed as a fraction of the average intensity ${ }^{[10]}$. The QPO amplitude is plotted in Figure 3.7 as the square root of the area in one logarithmic frequency interval, $\sqrt{\Delta P \Delta f}$, where $\Delta P$ is 1.96 times the standard error of the average Fourier power over the logarithmic frequency interval, $\log (\Delta f)$. The QPOs for this limit are contained within a given logarithmic frequency interval, giving a quality factor, $\mathrm{Q}>9$. Over the frequency range from 34 to $357 \mathrm{~Hz}$ where diskoseismic 


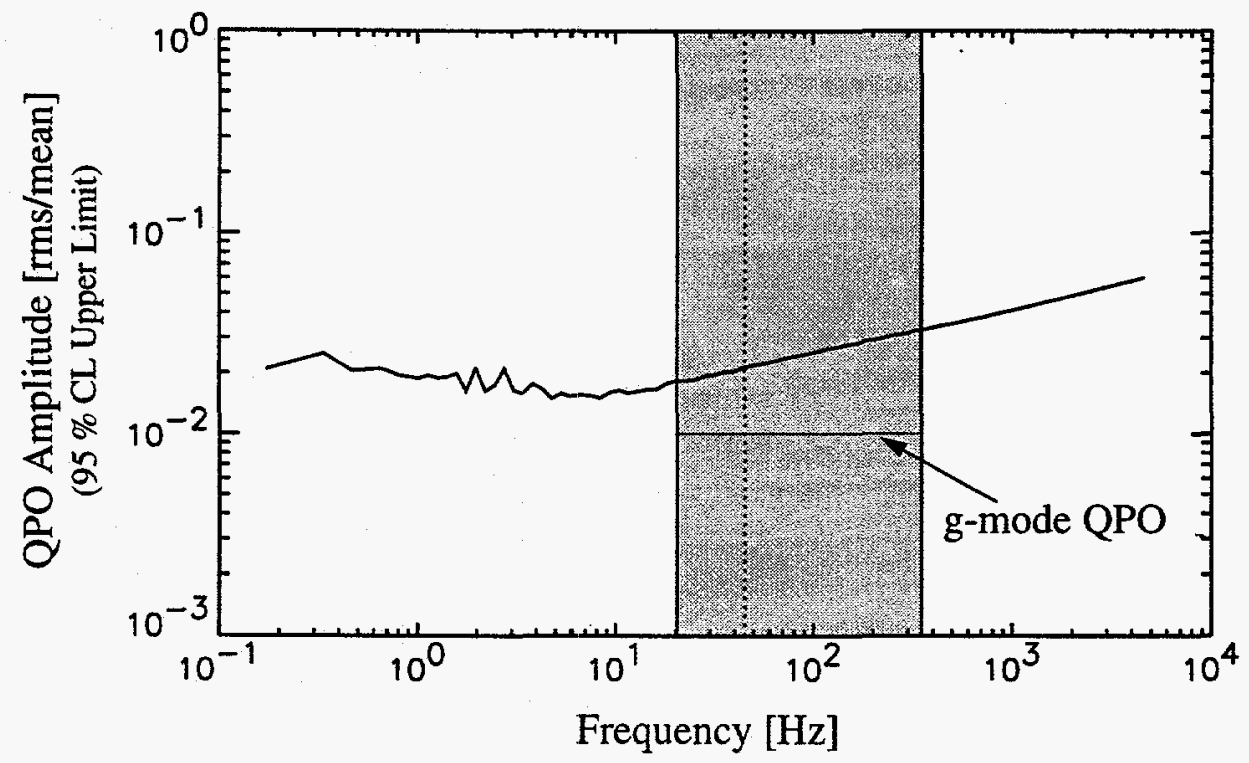

Fig. 3.7 The 95\% confidence level upper limit for detecting QPOs in Cyg X-1 from HEAO A-1 with a quality factor, $Q>9$ for a given fractional rms amplitude. The shaded region spans the range of expected frequencies for g-mode QPOs, with the horizontal line marking the expected gmode QPO amplitude and the dashed vertical line marking the frequency for a non-rotating, $16 M_{\odot}$ black hole.

QPOs are predicted for Cyg X-1, the fractional amplitudes are less than $1.8 \%$ to $3.3 \%$, respectively at a $95 \%$ confidence level. For a $16 M_{\odot}$ black hole, the limit is $2.2 \%$. The amount of HBR data on Cyg X-1 is insufficient to detect QPOs at the $1 \%$ fractional rms amplitudes predicted by relativistic diskoseismology ${ }^{[9]}$. 


\section{References}

1) Meekins, J. F., Wood, K. S., Hedler, R. L., Byram, E. T., Yentis, D. J., Chubb, T. A., \& Friedman, H.. (1984), ApJ, 278, 288.

2) Wood, K. S., et al. (1984), ApJS, 56, 507; Wood, K. S. (1978), “HEAO General Information, 2nd Edition", NRL internal report.

3) Anderson, G. \& Anderson, P. (1986), The UNIX C Shell Field Guide, Prentice-Hall, New Jersey.

4) Research System, Inc. (1994), IDL User's Guide, Research Systems, Inc., Boulder, CO.; (1994), IDL Reference Guide, Boulder, Co.: Research Systems, Inc.

5) Sun Microsystems, Inc. (1993), SunOS 5.3 Networking Interfaces Programmer's Guide, Sun Microsystems, Inc., Mountain View, CA.

6) Müller, J. W. (1973), Nucl. Instr. and Meth., 112, 47; (1974), Nucl. Instr. and Meth., 117, 401 .

7) Eadie, W. T., et al. (1971), Statistical Methods in Experimental Physics, NorthHolland Publishing Co., Amsterdam, 74.

8) Leahy, D. A.Leahy, D. A., Darbro, W., Elsner, R. F., Weisskopf, M. C., Sutherland, P. G., Kahn, S., \& Grindlay, J. E. (1983), ApJ, 266, 160.

9) Nowak, M. A. \& Wagoner, R. V. (1991), ApJ, 378, 656; (1992), ApJ, 393, 697; (1993), ApJ, 418, 187; (1995), MNRAS, 274, 37; Perez, C. A., Silbergleit, A.S., Wagoner, R. V., \& Lehr, D. E. (1997), ApJ, 476, 589.

10) Van der Klis, M. (1995), X-Ray Binaries, W.H.G. Lewin, J. V. Paradijs and E.P.J. Van den Heuvel, editors, Cambridge University Press, Cambridge, England, p. 259. 
Chapter 4

RXTE/PCA DATA STUDIES

\subsection{Introduction}

The RXTE/PCA instrument is capable of collecting X-ray data at time resolutions down to $1 \mu \mathrm{s}$ and up to 256 energy channels covering $2-60 \mathrm{keV}$. The time resolution capability of the instrument is the finest for all current and previous astronomical X-ray detectors. The data collected from this instrument represents the opportunity to explore the time variability of $\mathrm{X}$-ray objects to unprecedented submillisecond time scales.

\subsection{Description of the RXTE/PCA Data}

Under guest observation proposal D0889, P.I. P. Hertz, the RXTE/PCA instrument $^{[1]}$ observed Cygnus X-1 in its high state on June 8,1996 , for 23 minutes. The data mode for the Proportional Counter Array (PCA) was set to the E_4us_4B_0_1s configuration, giving a time resolution of 4 microseconds and 4 energy channels: (1.6 $6.4 \mathrm{keV}),(6.4-10.1 \mathrm{keV}),(10.1-14.6 \mathrm{keV})$ and $(14.6-99.6 \mathrm{keV})$.

The resulting data were written in Flexible Image Transport System (FITS) ${ }^{[2]}$ format. The components of this FITS file are described in Table 4.1 and further documented at the High. Energy Astrophysics Science Archive Research Center (HEASARC $^{[3]}$. There are only two basic FITS formats for the RXTE/PCA science data: 


\begin{tabular}{|l|l|}
\hline Name & Description \\
\hline Primary Header & $\begin{array}{l}\text { Information about the mission, the instrument, the observation and } \\
\text { the initial processing. }\end{array}$ \\
\hline Primary Image & Array is blank. \\
\hline $\begin{array}{l}\text { First Extension } \\
\text { Header }\end{array}$ & $\begin{array}{l}\text { Description of the contents of the first extension. For convenience, it } \\
\text { also contains some of the same information as the primary header. }\end{array}$ \\
\hline First Extension & $\begin{array}{l}\text { Contains the scientific data. In the case of science array files, the first } \\
\text { extension is called XTE_SA. In the case of science event files, it is } \\
\text { called XTE_SE. }\end{array}$ \\
\hline $\begin{array}{l}\text { Second Extension } \\
\text { Header }\end{array}$ & $\begin{array}{l}\text { Description of the contents of the second extension. For } \\
\text { convenience, it also contains some of the same information as the } \\
\text { primary header. }\end{array}$ \\
\hline Second Extension & $\begin{array}{l}\text { Lists the standard good time intervals, i.e. the start and stop times of } \\
\text { all the potentially useable data in the file. }\end{array}$ \\
\hline
\end{tabular}

Table 4.1 Description of the RXTE FITS format.

science array and science event. The science array format is used for data binned at regular intervals by the spacecraft electronics. For unbinned data (e.g., individual events collected in this observation), the science event format is used.

The science data occupy the XTE_SE extension as event words - binary-encoded descriptions of the individual events. In the XTE_SE extension the science data is arranged in rows (one per event) and in two columns: time and event word. Event words define the properties of each event with respect to a template of all possible properties within the data mode configuration (e.g. E_4us_4B_0_1s). This template is broken up into sections that, depending on the particular configuration, refer to items such as Proportional Counter Unit (PCU) Identification Number (ID), Pulse Height Analyzer (PHA) channel band, event overflow, etc. A detailed description of these templates can also be found at the HEASARC ${ }^{[4]}$.

\subsection{Processing the RXTE/PCA Data}

The tasks for the off-line analysis software are two-fold: extract good event times and save those event times in an easily retrievable manner. Table 4.2 summarizes the 


\begin{tabular}{|l|l|}
\hline \multicolumn{2}{|c|}{ OFF-LINE DATA PROCESSING STEPS } \\
\hline 1) FITSHDR & Extract the FITS headers \\
\hline 2) E4US4B01 & Decipher the Event words \\
\hline 3) XTETIMES & $\begin{array}{l}\text { Filter event times according to PCU ID and Energy } \\
\text { channel }\end{array}$ \\
\hline
\end{tabular}

Table 4.2 The major off-line processing steps for the RXTE/PCA data.

steps followed to complete these tasks. The RXTE/PCA data are written in a welldocumented FITS format, greatly simplifying the job of extracting good event times. The primary and extension headers are first extracted from the FITS file using the FITSHDR routine. In particular, the first extension header describes the templates used to encode the event words in the XTE_SE extension. The E4US4B01 routine uses this description to decipher the bits in the event word into various items such as the PCU ID and energy channel. This information is used to filter events according to their PCU ID and energy channels in the XTETIMES routine. All of the routines listed in Table 4.2 were written in the Interactive Data Language (IDL) ${ }^{[5]}$. In addition, several FITS routines from IDL Astronomy User's Library ${ }^{[6]}$ were also called to interpret the FITS file format.

The event times were separated according to their PCU ID and saved as 5 separate IDL Save Session files ${ }^{[5]}$, one for each PCU. Another processing run selected events from PCU 0 and separated events according to their energy channel.

Invalid events were removed in the XTETIMES routine by selecting various criteria in the first and second (XTE_SE) extensions of the FITS file. Any event times not within the good time intervals specified in the second extension were cut. For each row of the XTE_SE extension, the event word was checked for a valid time, PCU ID and energy channel. If any of those items was invalid, (e.g. because the row contained overflow information) the time recorded in the first column would be eliminated from the returned event times array. Finally, the event times from the first column were checked by verifying that the time encoded in its event word agreed. 


\begin{tabular}{|l|c|c|c|c|c|}
\hline PCU & 0 & 1 & 2 & 3 & 4 \\
\hline Dead Time $[ \pm 0.03 \mu \mathrm{s}]$ & 8.77 & 8.74 & 8.87 & 8.81 & 8.81 \\
\hline
\end{tabular}

Table 4.3 Non-paralyzable dead times for each PCU determined from fitting [3.1] to the RXTE/PCA waiting time distributions.

\subsection{Instrumental Effects}

Distortions caused by instrumental effects dominated the time variability of the HEAO A-1 HBR data. Given that proven example of how important instrumental effects can be, emphasis was placed on searching for possible instrumental effects in the RXTE/PCA data. The dead times were first determined by separately fitting [3.1] to the waiting time distribution of each PCU using the method described in Section 3.4.1. As in the HEAO A-1 HBR data, the differences between the two types of dead time, nonparalyzable and paralyzable, were negligible. A non-paralyzable dead time was chosen in the fits because of its mathematical simplicity. The results of these fits, shown in Table 4.3 give an average dead time of 9 microseconds.

Additional instrumental effects were searched for by analyzing RXTE/PCA data on a Poisson source, the Crab Nebula. The data archive in the RXTE Guest Observer Facility ${ }^{[4]}$ was searched for public data on observations of the Crab. Several such observations at a one millisecond time resolution were located and subsequently analyzed for instrumental effects. The Fourier power spectra were summed and averaged over all observations and over all five PCUs. Figure 4.1 shows the final averaged Fourier power spectrum of all the Crab data analyzed. The Fourier powers at the harmonics of the Crab pulsar frequency $(33 \mathrm{~ms})^{-1}$ were excluded from the plot. As discussed in the next Section, the expected Poisson noise floor shown by the solid horizontal line fits the Fourier power spectrum of the Crab exceptionally well. No distortions of the power spectrum beyond those due to dead time were detected in this Crab data for all frequencies up to the Nyquist value, $500 \mathrm{~Hz}$. 


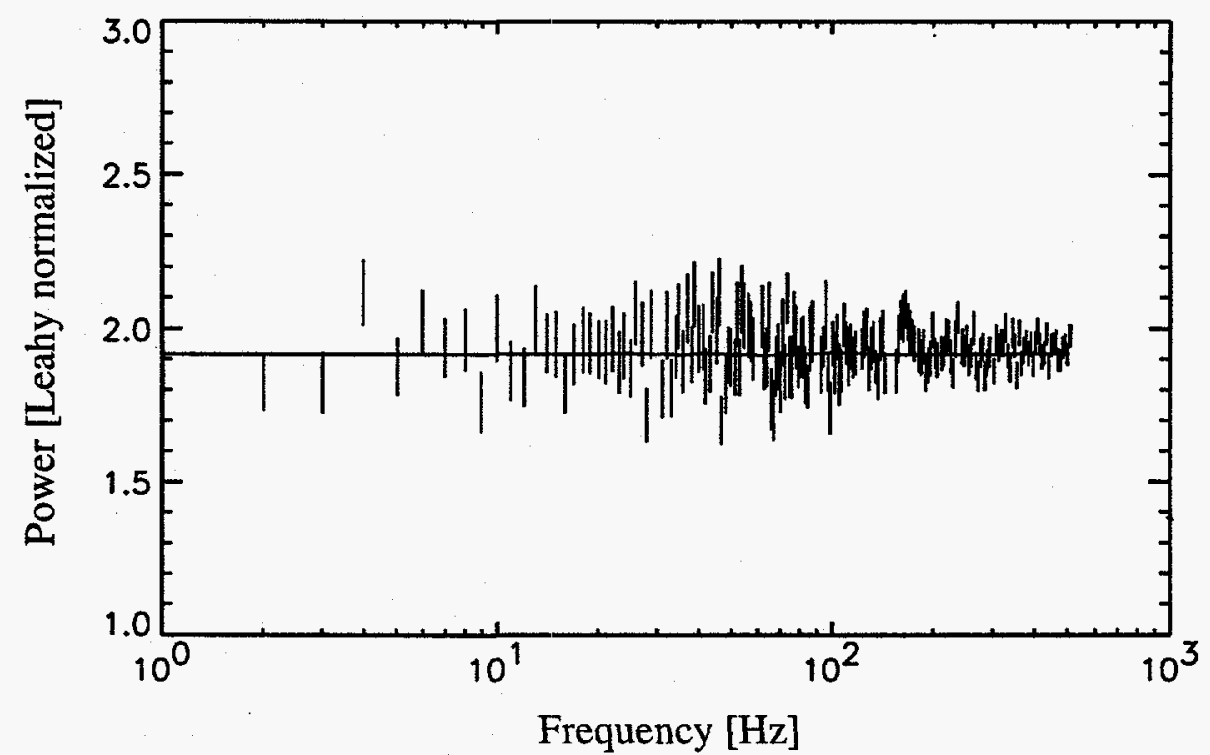

Fig. 4.1 The Fourier power spectrum of the RXTE/PCA data on the Crab, excluding values at the harmonics of the Crab pulsar frequency. The solid horizontal line is the expected Poisson noise floor corrected for RXTE/PCA dead time. The fit of the expected Poisson noise floor to the Crab spectrum shown gives a goodness of fit: $\chi^{2} /$ dof $=173 / 178$.

\subsection{Data Analysis}

The time variability analysis of the RXTE/PCA data was greatly facilitated by the limited number of instrumental effects introduced by the detector. The analysis of Crab data gave no indication of any other instrumental effect besides that due to detector dead time. Correcting the Poisson noise floor only for dead time is straightforward and well understood. Given confidence that all of the instrumental distortions of the Poisson noise floor are understood, an upper limit to measuring noise-subtracted power can be determined.

\subsubsection{Correcting the Poisson Noise Floor}

In the presence of dead time, the Poisson noise floor is affected by the length of the dead time and the observed count rate of the X-ray source ${ }^{[7]}$. This Poisson noise floor can be determined by first generating simulated Poisson data distorted by dead time, and then calculating the Fourier spectrum of that simulated data. The dead time and the true 
count rate are input parameters into [3.1] to define the probability function of waiting times between successive Poisson events. The true count rate is determined from the observed count rate and the dead time by using the formula listed in Table 3.3. Random waiting times are drawn from this probability function until the total number of events equals that of the data. The generated waiting times are accumulated into event times and then the simulated event times are Fourier analyzed in the same manner as the data. The resulting average Fourier spectrum defines the Poisson noise floor in the presence of dead time for that data.

\subsubsection{Frequency Upper Limit on Detected Intrinsic Variability}

Poisson noise in the presence of dead time is well understood in terms of a binned offset exponential, [3.1] that describes the probability of waiting times between events. Given this understanding, the highest frequency that Fourier power can be detected above the Poisson noise can be determined for a given confidence level. Signal is detected above the noise at a $95 \%$ confidence level or greater when the noise-subtracted power exceeds 1.96 times the standard deviation of the power defining the corrected Poisson noise floor. The frequency upper limit is defined as the highest frequency where signal is detected over the noise at a given confidence level.

\subsection{Results}

Given the technique described in the previous Section, the RXTE/PCA data can now be corrected for instrumental effects. For each PCU, the data were summed over all energy channels and then the resulting light curves were Fourier analyzed. The corrected Poisson noise floor was simulated for each PCU using the average count rate and dead time specific to that PCU. To further improve the statistics, the Fourier spectra of all the PCUs were averaged together. Figure 4.2 shows the resulting power spectra for Cyg X-1. The corrected Poisson noise floor is flat with a small frequency dependency near the Nyquist frequency. The level is lowered by $2 \%$ from its value when no instrumental effects are present. 


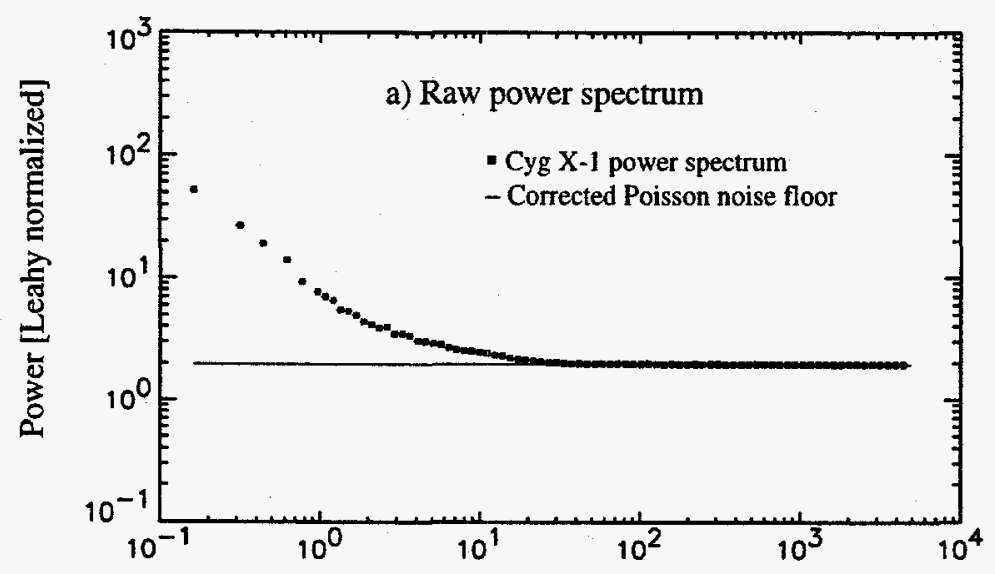

Frequency [Hz]

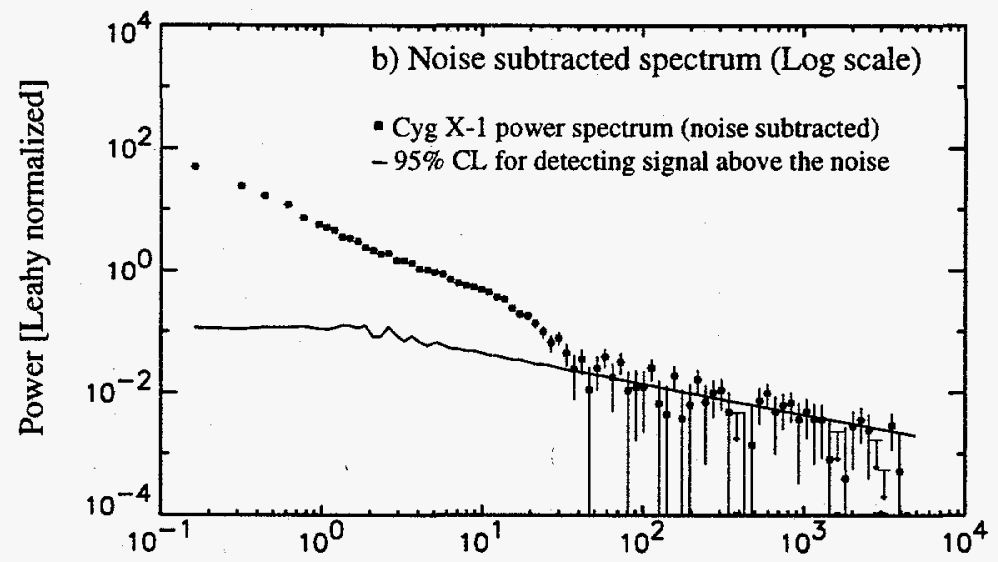

Frequency $[\mathrm{Hz}]$

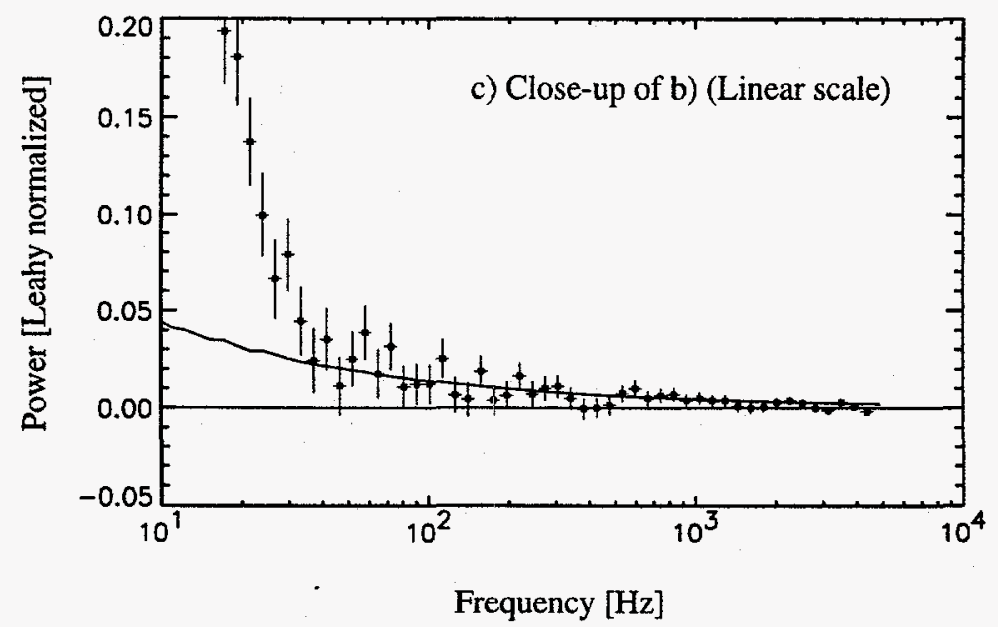

Fig. 4.2 The a) raw and noise-subtracted Fourier power spectrum of Cyg X-1 from the RXTE/PCA data in b) full and c) close-up views. The Poisson noise floor was corrected for instrumental effects. The solid curve represents the $95 \%$ confidence level for detecting a signal above the Poisson noise floor. 


\begin{tabular}{|l|c|c|c|c|c|c|}
\cline { 2 - 7 } \multicolumn{1}{c|}{} & \multicolumn{2}{|c|}{ Power Law Before Apparent Break } & \multicolumn{3}{|c|}{ Power Law After Apparent Break } \\
\hline Detector & $\begin{array}{c}\text { Frequency } \\
\text { Range } \\
{[\mathrm{Hz}]}\end{array}$ & $\begin{array}{c}\text { Photon } \\
\text { Index }\end{array}$ & $\chi^{2} /$ dof & $\begin{array}{c}\text { Frequency } \\
\text { Range } \\
{[\mathrm{Hz}]}\end{array}$ & $\begin{array}{c}\text { Photon } \\
\text { Index }\end{array}$ & $\chi^{2} /$ dof \\
\hline RXTE/PCA & $0.1-3$ & $1.24 \pm 0.04$ & $28 / 14$ & $14-40$ & $2.1 \pm 0.5$ & $4 / 8$ \\
\hline
\end{tabular}

Table 4.4 Summary of power law fits to the noise-subtracted Fourier power spectrum. The break between the two different power laws occurs between $10-20 \mathrm{~Hz}$.

As in the HEAO A-1 data, the noise-subtracted power spectrum also shows two power laws in the spectrum. The break between the two power laws occurs at a higher frequency than the previous data set, between 10 to $20 \mathrm{~Hz}$. In addition, the break is more rounded. Table 4.4 summarizes the results of fitting power laws to the regions before and after this break.

The 95\% confidence level for detecting a signal above the Poisson noise floor is also plotted in Figures $4.2 \mathrm{~b}$ and $4.2 \mathrm{c}$. At frequencies greater than $50 \mathrm{~Hz}$, the effect of approaching the Poisson noise floor is demonstrated by a flattening of the spectrum. It is an artifact introduced by limited counting statistics. Sensitivity to measuring intrinsic variability is limited by the variance of the noise floor. With additional data, the $95 \%$ confidence level curve will be lowered to reveal how the spectrum continues beyond $50 \mathrm{~Hz}$.

The downward slope seen at frequencies greater than $50 \mathrm{~Hz}$ is a consequence of averaging over equal logarithmic frequency intervals. The number of frequencies, $M$ averaged over in an equal logarithmic frequency interval is proportional to the central frequency of that interval. Thus, $M$ increases while the standard deviation decreases as $1 / \sqrt{M}$. Despite the limiting statistics, evidence for marginal excess power is still seen at frequencies between $100-4000 \mathrm{~Hz}$. Over $33 \%$ of the excess powers at frequencies greater than $100 \mathrm{~Hz}$ exceed the $95 \%$ confidence level curve.

Again, as in the HEAO A-1 data, no evidence is seen for QPOs. A better 95\% confidence level upper limit can be obtained with the RXTE/PCA data since the data set 


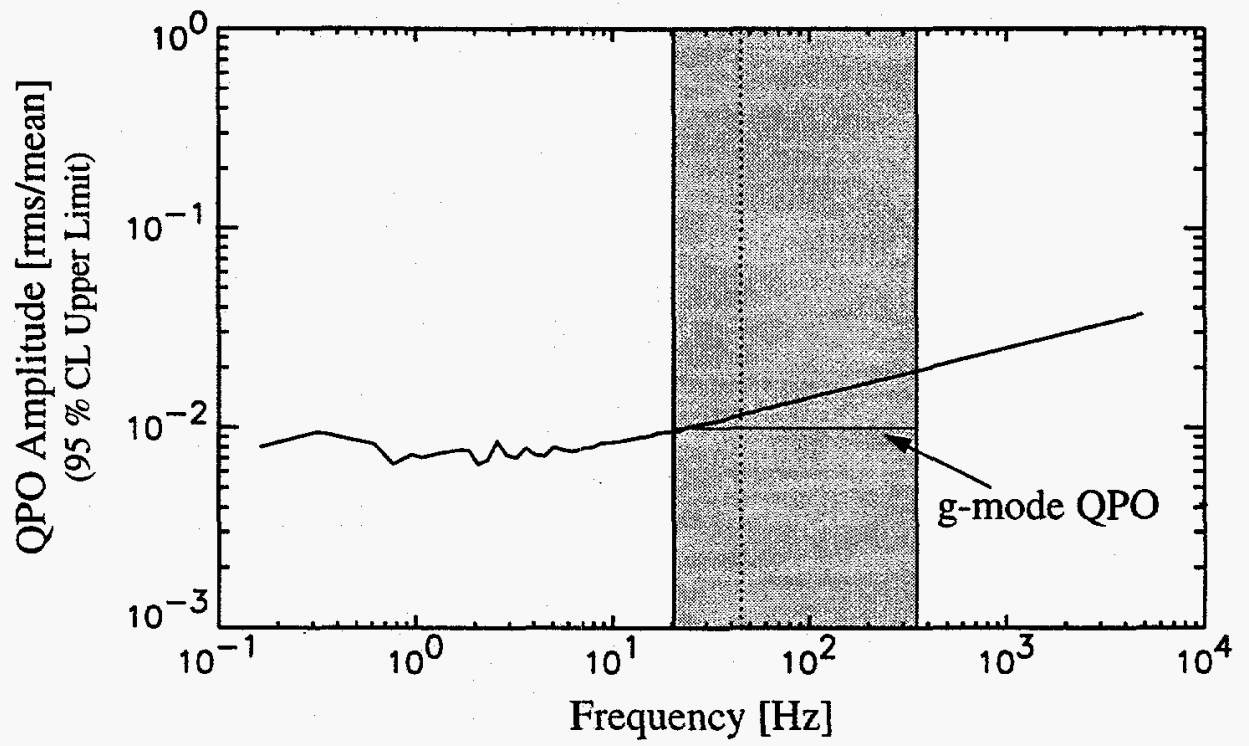

Fig. 4.3 The 95\% confidence level upper limit for detecting QPOs in Cyg X-1 from RXTE/PCA with a quality factor, $\mathrm{Q}>9$ for a given fractional $\mathrm{rms}$ amplitude. The shaded region spans the range of expected frequencies for g-mode QPOs, with the horizontal line marking the expected gmode QPO amplitude and the dashed vertical line marking the frequency for a non-rotating, $16 M_{\odot}$ black hole.

contained 6 times as many photons as in the HEAO A-1 data. Figure 4.3 shows fractional rms amplitudes ranging from $0.96 \%$ to $1.9 \%$ over the frequencies where QPOs are predicted by relativistic diskoseismology ${ }^{[8]}$. Diskoseismic QPOs with $1 \%$ fractional rms amplitudes can be excluded for frequencies $\leq 25 \mathrm{~Hz}$ at a $95 \%$ confidence level. The excluded frequencies contained within the shaded region in Figure 4.3 correspond to retrograde orbits with black hole angular momenta parameter $a \leq-0.5$. At frequencies greater than $25 \mathrm{~Hz}$, the QPO amplitude limits are higher than the $1 \%$ fractional rms amplitudes predicted for diskoseismic QPOs over the range of expected frequencies for Cyg X-1. 


\section{References}

1) Jahoda, K., et al. (1996), SPIE, 2808, 59; Rhee, H. J. (1997), "Proportional Counter Array (PCA)", http://Iheawww.gsfc.nasa.gov/docs/xray/xte/pca/.

2) Hanisch, R. J. et al. (1995), "Definition of the Flexible Image Transport System (FITS )", NOST 100-1.1; Schlesinger, B. M., "FITS Support Office Home Page", http://www.gsfc.nasa.gov/astro/fits/fits_home.html.

3) White, N. E., et al. (1997), "HEASARC High Energy Astrophysics Science Archive Research Center", http: //heasarc.gsfc.nasa.gov/.

4) Swank, J.H., et al. (1997), "RXTE Guest Observer Facility", http://heasarc.gsfc.nasa.gov/docs/xte/xte_1st.html.

5) Research System, Inc. (1994), IDL User's Guide, Boulder, Co.: Research Systems, Inc.; 1994), IDL Reference Guide, Boulder, Co.: Research Systems, Inc.

6) Landsman, W. (1997), "The IDL Astronomy User's Library", http://idlastro.gsfc. nasa.gov/homepage.html.

7) Zhang, W., Jahoda, K., Swank, J. H., Morgan, E. H., \& Giles, A. B. (1995), ApJ, 449, 930.

8) Nowak, M. A. \& Wagoner, R. V. (1991), ApJ, 378, 656; (1992), ApJ, 393, 697; (1993), ApJ, 418, 187; (1995), MNRAS, 274, 37; Perez, C. A., Silbergleit, A.S., Wagoner, R. V., \& Lehr, D. E. (1997), ApJ, 476, 589. 
Appendix A

\section{Format of the HEAO A-1 High-Bit-Rate Data}

\section{A.1 Bit Telemetry Mode Format}

The HEAO A-1 HBR data in the Bit telemetry mode can be basically described as a continuous stream of bits, each bit representing a $7.8125(\approx 8) \mu$ s time bin set if one or more events occurred in that interval. The normal (NRZ) telemetry data was written along with the HBR data onto analog tapes that were later digitized at NRL. The format of the digitized data was described in a Data General assembly routine developed at NRL. This routine was later translated to an IDL routine with the same name, HBRSYNC.

The digitized data consists of fixed length records of 8220 bytes. Each file record spans a minor frame, a period of $320 \mathrm{~ms}$. Table A.1 gives a breakdown of a file record where a word is defined as 2 bytes or 16 bits. The majority of the file record forms an HBR data record, a continous block of 4096 words. Each word of an HBR data record spans an $80 \mu$ s period, one bit for each $8 \mu$ s time bin. A set bit represents data presence in that corresponding time bin. Table A. 2 describes the bit format for each of these words.

The start of an HBR data record is offset from the start of a minor frame of the NRZ data. This offset is typically 65 words. However, this offset may vary from file to file and even between HBR data records if any errors are present. 


\begin{tabular}{|l|l|}
\hline Word(s) & Description \\
\hline 0 & $\begin{array}{l}\text { Time information. The minor frame number can be extracted from this } \\
\text { word. }\end{array}$ \\
\hline $1-4096$ & HBR data record. \\
\hline 4097 & NOVA tape sequence number \\
\hline 4098 & NOVA tape run number \\
\hline 4099 & Number of NOVA files \\
\hline 4100 & NOVA tape file number \\
\hline 4101 & Copy error flag \\
\hline 4102 & NOVA input record size \\
\hline $4103-4105$ & Date of copy (year, day, month) \\
\hline $4106-4108$ & Time of copy (hour, minutes, seconds ) \\
\hline 4109 & Undefined \\
\hline
\end{tabular}

Table A.1 Contents of a HBR File record.

\begin{tabular}{|c|c|}
\hline Bit(s) & Description \\
\hline $0-5$ & HBR photon data at corresponding relative time bins: $[9,8,7,6,5,4]$ \\
\hline 6 & $6.4 \mathrm{kbs}$ synch lock flag $(1=$ lost $)$ \\
\hline 7 & $\begin{array}{l}\text { Frame synch flag from the DCS ground station. }(0=\text { DCS ground station is } \\
\text { locked on and has found the minor frame synch word. For each minor frame, } \\
\text { this bit }=0 \text { for } 16 \text { sequential words and }=1 \text { for all other words.) }\end{array}$ \\
\hline $8-11$ & HBR photon data at corresponding relative time bins: $[3,2,1,0]$ \\
\hline 12 & $\begin{array}{l}6.4 \mathrm{kbs} N R Z \text { data (There are two copies of } 128 \mathrm{NRZ} \text { data words that are } \\
\text { embedded into each HBR data record. Each copy is formed from } \\
\text { concatenating bit } 12 \text { of every other word in an HBR data record, starting at } \\
\text { the Most Significant Bit of the NRZ word. For example, if HBR data words } \\
20,22 \text { and } 24 \text { contain bits } 15,14 \text { and } 13 \text { of a NRZ word then HBR data } \\
\text { words } 21,23 \text { and } 25 \text { would also contain these same three NRZ bits.) }\end{array}$ \\
\hline 13 & $\begin{array}{l}\text { Tick tock bit for copies of NRZ data (Namely, this bit "tick-tocks" between } 0 \\
\text { and } 1 \text { for sequential HBR data words. The words where this bit is set to } 0 \text { or } \\
1 \text { have the first or second copy of the NRZ data, respectively) }\end{array}$ \\
\hline 14 & High bit rate lock $(1=$ lost $)$ \\
\hline 15 & Analog lost data bit $(1=$ lost $)$ \\
\hline
\end{tabular}

Table A.2 Format of the bits in a given HBR data word. Bit $0=$ Least Significant Bit. 


\begin{tabular}{|l|l|l|}
\hline Name & $\begin{array}{c}\text { Number of } \\
\text { records }\end{array}$ & Description \\
\hline MJF header & 1 & Overall accounting statistics and errors for this MJF. \\
\hline NRZ Data block & 32 & The normal $6.4 \mathrm{kbps}$ telemetry data for this MJF. \\
\hline HBR Data block & Dynamic & The $128 \mathrm{kbps}$ HBR data for this MJF. \\
\hline
\end{tabular}

Table A.3 Structure of a Major Frame segment.

Several steps are taken to determine this offset. In an HBR data word, the frame synch bit (bit 7) is cleared when the DCS ground station has found the minor frame synch word and is locked on. The HBR data record is searched for the first word where the frame synch bit is cleared. This typically occurs at the last word of the HBR data record.

The actual start of the minor frame occurs at an additional offset of typically, -64 words. Namely, a minor frame usually starts at word 4031 of the HBR data record, (starting from word 0 ). To verify that the minor frame starts at this offset, a synch pattern is searched for in the first 24 bits of the NRZ data starting from the given offset. Since a copy of the NRZ data occurs in bit 12 of every other HBR word, these 24 bits actually span 48 words of the HBR data record. The synch pattern searched for is:

\section{0}

where the right-most bit represents the Least Significant Bit.

If the 24 bits of NRZ data match the synch pattern then we have found the beginning of the minor frame for both the NRZ and the HBR data. Otherwise, this negative offset is varied between 0 and -100 with the 24 bit synch pattern searched for at each offset until a match is found.

\section{A.2 Photon Time Interval Format}

The Photon Time Interval (PTI) format organizes the HBR data into sequential segments of data, 40.96 seconds long. This period of time is called a major frame, MJF and each major frame may be divided into 128 minor frames. Each major frame segment consists of fixed-length records of 1024 bytes and each record may be read into a 512 


\begin{tabular}{|l|l|l|}
\hline $\begin{array}{l}\text { Word } \\
\text { Offset }\end{array}$ & $\begin{array}{l}\text { Pointer } \\
\text { Name }\end{array}$ & Description \\
\hline 0 & MJF & MJF number calculated (32-bit integer) \\
\hline 2 & MJFNRZ & MJF number read (32-bit integer) \\
\hline 4 & MJFLAGS & MJF flags (16 bits) (see Table A.5) \\
\hline 5 & SYNLOSS & Total number of HBR sync loss errors (32-bit integer) \\
\hline 7 & CLKERRS & Total number of Clock TicToc errors (32-bit integer) \\
\hline 9 & MJFCNTS & Total counts (32-bit integer) \\
\hline 11 & MNFMISS & Number of Missing MNF's \\
\hline 12 & MNFLAGS & MNF flags (duplicated) \\
\hline 139 & MNFNDTS & Number of photon time intervals (Counts-1)/MNF \\
\hline 266 & DATRECS & Total number of data records written \\
\hline 267 & DATRECN & Data record number for each MNF \\
\hline 394 & PARERRS & Total number of parity errors \\
\hline 395 & SYNMNFS & Number of MNF's with HBR sync loss errors \\
\hline 396 & NOSYNCH & Number of buffers with no DCS synch bit transition error(s) \\
\hline 397 & BADSYNC & Number of buffers with bad or missing synch pattern error(s) \\
\hline 398 & TIKTOK & Number of buffers with Clock TicToc errors \\
\hline 399 & BUMSIZ & Number of buffers with MNF is not 4096 words long error(s) \\
\hline 400 & BITSBAD & Number of buffers with DCS bit lock lost somewhere in MNF \\
\hline 401 & OBOUNDS & $\begin{array}{l}\text { Number of buffers with MNF extends beyond buffer size } \\
\text { error(s) }\end{array}$ \\
\hline 448 & MJFMARK & MJF marker (64 words) \\
\hline
\end{tabular}

Table A.4 Description of the Major Frame Header.

element, 16-bit integer array. The time interval or waiting times between events are stored in this data format instead of the absolute times as in the A-1 Bit mode data format.

The data structure of a MJF segment is broken into three sections: a header, a block of normal telemetry data (NRZ) and a block of HBR data as illustrated in Table A.3. The header provides an overall description of the current major frame segment with overall statistics ranging from the number and types of errors found to the number of 


\begin{tabular}{|l|l|l|}
\hline Bit & Bit Name & Description \\
\hline 0 & MJF_GDVS & Global data validity (0=ok) \\
\hline 1 & MNF_MISS & MNF(s) missing \\
\hline 2 & NO_DATAS & No events (data) detected \\
\hline 3 & SYN_ERRS & HBR sync loss error(s) \\
\hline 4 & PAR_ERRS & Tape parity error(s) \\
\hline 5 & HBR_FMTS & HBR format (0=bit,1=word) \\
\hline 6 & NOSYN_ERRS & No DCS synch bit transition error(s) \\
\hline 7 & BADSYN_ERRS & Bad or missing synch pattern error(s) \\
\hline 8 & CLK_ERRS & Clock tictoc error(s) \\
\hline 9 & BUMSIZ_ERRS & MNF is not 4096 words long error(s) \\
\hline 10 & BITBAD_ERRS & DCS bit lock lost somewhere in MNF error(s) \\
\hline 11 & OBOUND_ERRS & MNF extends beyond buffer size error(s) \\
\hline
\end{tabular}

Table A.5 Bit assignments for the MJF flags word, MJFLAGS. ( $0=\mathrm{LSB})$

events detected. A complete description of this header is given in Tables A.4 and A.5.

Although these headers maintain the bookkeeping of errors found in the HBR data, safeguards are needed to check the integrity of the PTI data itself. Data integrity may be breached by file corruption or read errors. To address this issue a marker is placed in the last 64 words of every MJF header. This marker is the number sequence: [66, 79, $75,83,84,69,86,69]$ repeated 8 times.

Even if there are no events in a given MJF or if a MJF is missing, a MJF header is still written out. Information in the header will reflect this by indicating that there are no counts and additionally, for the case of a missing MJF that there are 128 missing minor frames.

The NRZ Data block follows the MJF header and contains 32 records (512 words/record) of normal $6.4 \mathrm{kbps}$ telemetry data from all four HEAO 1 instruments. This block contains a total 16384 or $128^{2}$ words. Each sequential 128 words represents a sequential minor frame of NRZ data. There is a caveat in the case when there are one or more missing minor frames in the HBR data. If a missing minor frame occurs, then by 


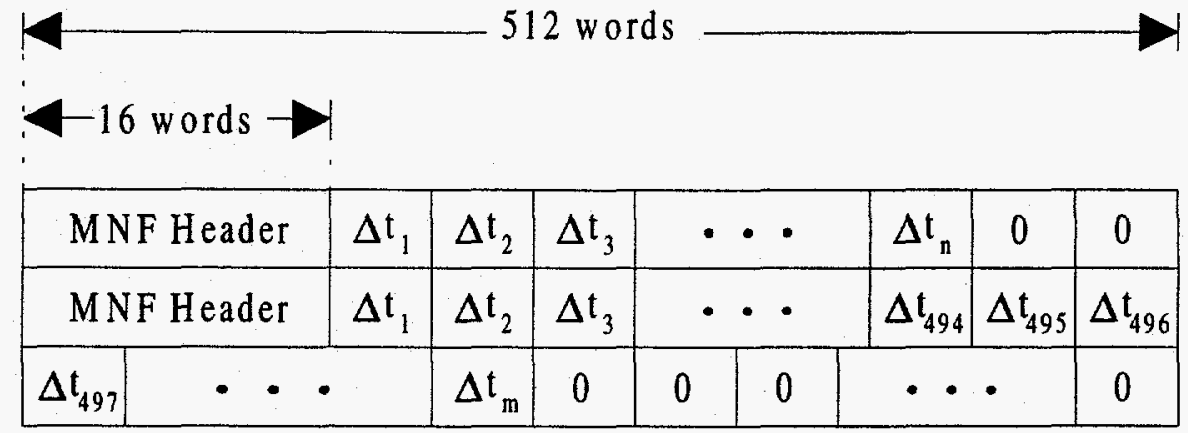

Fig. A.1 Example of two MNF sections in the an HBR Data block.

definition there is no corresponding NRZ data. Namely, 128 words of the NRZ Data block are zero. This and any additional sections of zero NRZ data are positioned at the end of the NRZ Data block regardless of which minor frames are missing.

Unlike the MJF header and the NRZ Data block, the size of the HBR Data block is dynamic in terms of its total number of records. While the HBR Data block may be divided into minor frame (MNF) sections as in the case of the NRZ Data block, each of these minor frame sections may have different numbers of records. Each minor frame section consists of a 16-word minor frame header followed by a list of successive waiting times between events. Therefore, the number of records for a minor frame section depends on the number of waiting times in that minor frame. The situation is further illustrated in Figure A.1.

Each minor frame header also has a marker. This provides another means of testing the integrity of the PTI data. The number sequence: $[-11337,2179]$ is placed in the last two words of the header.

Missing minor frames are also accounted for in the HBR Data block. A MNF header is written out for each range of missing minor frames. The remaining 496 words in the record that holds such a MNF header are flushed to 0 . The missing MNF bit is set and the range of missing MNFs is stored in the MNF header. For further descriptions of the MNF header, see Tables A.6 and A.7. 


\begin{tabular}{|l|l|l|}
\hline $\begin{array}{l}\text { Word } \\
\text { Offset }\end{array}$ & $\begin{array}{l}\text { Pointer } \\
\text { Name }\end{array}$ & Description \\
\hline 0 & MNF & MNF number calculated/first missing \\
\hline 1 & MNFNRZ & MNF number read/last missing \\
\hline 2 & MNFLAG & MNF flags (16 bits) (see Table 7) \\
\hline 3 & MNFCNT & Counts this MNF \\
\hline 4 & NRZOFF & NRZ data offset in buffers \\
\hline 5 & SYNLOS & Number or HBR sync loss errors \\
\hline 6 & CLKERR & Number of Clock TicToc errors \\
\hline 7 & DATREC & Data record number \\
\hline 8 & MNFNDT & Number of photon time intervals in this MNF \\
\hline 9 & MNFLDT & Number of photon time intervals in the last MNF \\
\hline 10 & DELBEG & Time between beginning of MNF to first photon \\
\hline 11 & DELEND & Time between last photon to end of MNF \\
\hline 12 & SYNFLG & HBRsync flags (16 bits) \\
\hline 13 & ERROFF & Error offset in buffers \\
\hline 14 & MNFMARK & MNF marker (32-bit integer) \\
\hline
\end{tabular}

Table A.6 Description of the MNF Header

\begin{tabular}{|l|l|l|}
\hline Bit & Bit Name & Description \\
\hline 0 & MNF_GDV & Global data validity $(0=\mathrm{OK})$ \\
\hline 1 & MNF_MIS & MNF missing \\
\hline 2 & NO_DATA & No events (data) detected \\
\hline 3 & SYN_ERR & HBR sync loss error(s) \\
\hline 4 & CLK_ERR & Clock tictoc error(s) \\
\hline 5 & PAR_ER1 & Tape parity error buffer 1 \\
\hline 6 & PAR_ER2 & Tape parity error buffer 2 \\
\hline 7 & SUSPECT & $\begin{array}{l}\text { Current MNF suspect due to one or more sync } \\
\text { errors between current and previous MNFs }\end{array}$ \\
\hline
\end{tabular}

Table A.7 Bit assignments for the MNF flags word, MNFLAG. (0=LSB) 


\begin{tabular}{|l|l|}
\hline Tag Name & Description \\
\hline Name & Name associated with this HBR data file, SSS_TT_FF, [string]. \\
\hline Origin & Structure describing the origins of this save file. It tags are as follows: \\
Routine : Name of the IDL routine, 'CATPTI.PRO' \\
$\begin{array}{l}\text { Date } \quad \text { : Date of creation, SYSTIME(), [string] } \\
\text { Version : Version of IDL, copy of !VERSION } \\
\text { File } \quad \text { : Filename of the PTI file, including path, [string]. }\end{array}$ \\
\hline MJFH & Array of all the MJF headers, [intarr(512, nMJF)]. \\
\hline NRZ & Array of all the NRZ blocks, [intarr(128, nNRZ)]. \\
\hline MNFH & Array of all the MNF headers, [intarr(16, nMNF)]. \\
\hline PTI & $\begin{array}{l}\text { Array of all the Photon Time Intervals with the Adjacent Photon Events } \\
\text { removed, [intarr(nPTI)]. }\end{array}$ \\
\hline ts & $\begin{array}{l}\text { Array of all absolute photon times, [lonarr(nPTI+1)]. ts(0) = time of the } \\
\text { first photon relative to the beginning of it MNF. }\end{array}$ \\
\hline MJFs & Array of MJF numbers, [lonarr(nMJF)]. \\
\hline nMJF & Total number of MJFs, [fix]. \\
\hline nMNF & Total number of MNFs, [fix]. \\
\hline nPTI & Total number of PTIs, [long]. \\
\hline nAPE & Number of Adjacent Photon Events found/MJF, [intarr(nMJF)]. \\
\hline DBS & $\begin{array}{l}\text { Structure containing the HEAO HBR database information on this } \\
\text { target. }\end{array}$ \\
\hline GDV & Overall Global Data Validity flag (0=Ok), [fix]. \\
\hline
\end{tabular}

Table A.8 Description of the cat structure variable.

There is a caveat with regard to the values of the waiting times. The largest positive number a 16-bit integer can hold is 32767 . This is smaller than the largest possible waiting time within a minor frame, 40960. However, the MSB (the sign bit) effectively expands the range of numbers to [0-65535]. Therefore, any waiting times $>$ 32767 overflow to their negative complement, namely, $-2^{16}+$ waiting time. All waiting times in the range $\in[32768,40960]$ are mapped to their negative overflow values, $[-32768,-24576]$. 


\section{A.3 Concatenated Time Interval Format}

The HBR data that has been reformatted into the Photon Time Interval (PTI) format is organized into sequential blocks of Major Frames. This imposes a strong preference to analyze the event times in 40.96 second segments. To eliminate this bias and simplify access to all the event times, these Major Frame blocks of data are concatenated into arrays that are stored into one IDL structure variable.

Each array stored into an $\mathrm{IDL}$ structure variable must have a name or tag. A properly chosen tag name provides self-documentation of the data stored in that array. The IDL structure variable is then saved as an IDL Save Session file into a platform independent data format called eXternal Data Representation.

The routine needed to restore these files is built into IDL so the user is not dependent on a specific external routine that can properly interpret the data format. The name of the structure variable saved is called cat. Table A.8 lists the tags defined in this structure variable and provides a description of each.

\section{A.4 Relevant IDL Routines}

The following are listings of IDL routines that were developed at SLAC to read in the various $H B R$ data formats described in the last three Sections.

\section{A.4.1 Bit Telemetry Mode Format}

;+

; NAME:

; HBRSYNC

;

; PURPOSE:

This routine processes the HBRB data (High-Bit-Rate in Bit telemetry mode) and extracts the $128 \mathrm{kbps}$ and $6.4 \mathrm{kbps}$ datastream for a minor frame.

; CATEGORY:

HEAO HBR

; CALLING SEQUENCE:

; HBRSYNC, Buf [, HBR, NRZ, MJF, MNF, Flags] 


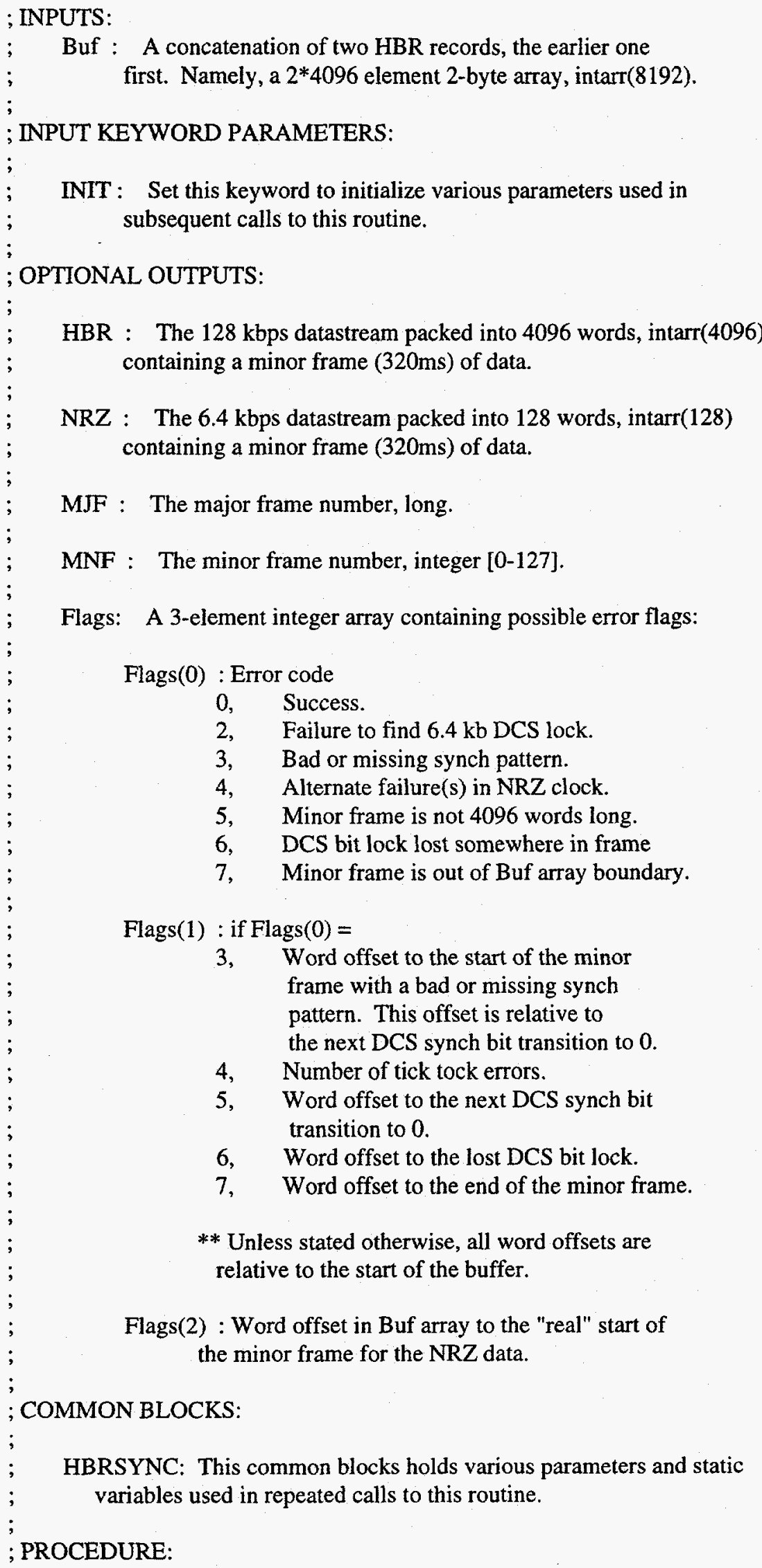


; You must call first this routine with the INIT keyword set to define

; various parameters.

;

; MODIFICATION HISTORY:

;

; MODIFIED 33183 TO CORRECT FOR OBSERVED TICKTOCK ERRORS ON SOME TAPES

; INIT ENTRY ADDED.

; MODULE TO CONTAIN BIT SHOVING STUFF FOR HEAO HIGH BIT RATE DATA

; D P MCNUTT 12683 - NO RIGHTS RESERVED

;

; THE FOLLOWING ASSEMBLY PARAMETER AFFECTS THE TIMING OF THE

; MINOR FRAME DATA RETURNED. FAZEF=0 WILL USE THE EARLIER OF

; THE TWO AVAILABLE COPIES OF A MINOR FRAME, FAZEF=1 WILL USE

; THE LATER.

; **** I will use the convention that BIT 0 is the LEAST significant bit.

$* * * *$ All numbers will be in base 10 , unless stated otherwise.

We will assume that the data is in BIG Endian convention since this

routine was written for the Data Generals which used this convention.

"Ported" to IDL from Data

General Eclipse assembly : Han Wen, April 1995.

23-APR-1995 Bugfix: NRZ was returning as a long integer (4-byte)

instead of a (2-byte) integer.

08-JUL-1995 Changed word offset (Flags(1)) for BITSBAD error

relative to start of buffer instead of start of minor

frame.

$;-$

pro HBRSYNC, Buf, HBR, NRZ, MJF, MNF, Flags, INIT=Init

common HBRSYNC, $B_{-}, E_{-}, V_{-}, C_{-}$

if keyword_set(INIT) then begin

; Define various bit parameters

$B_{-}=\{\$$

LOSS: $15, \$$; Analog lost data bit

HLOK:14,\$ ; High bit rate lock

TIKT:13,\$; Tick tock FAZEF bit for copies of NRZ data

LNRZ: $12, \$ ; 6.4 \mathrm{~kb}$ NRZ data

FSYN:7, \$; Frame synch flag from the DCS ground station

LLOK:6 $\$ ; 6.4 \mathrm{~kb}$ bit synch lock flag

HBR $1: 11, \$$

HBR2:8, \$ ; Bit locations in source words of HBR data bytes

HBR3:5, \$

HBR 4:0 \}

; Define error codes for the Flags array

$E_{-}=\{\$$

NOSYNCH $: 2, \$ ;$ Failure to find $6.4 \mathrm{~kb}$ DCS lock

BADSYNCH:3,\$ ; Bad or missing synch pattern 
; second word is SLIP value

TIKTOK $: 4, \$$; Alternate failure(s) in NRZ clock

; second word is count thereof

BUMSIZ :5,\$ ; Minor frame is not 4096 words long

; second word is ordinal of next synch

; bit transition if it occurred early.

BITSBAD :6,\$ ; DCS bit lock lost somewhere in the frame

OBOUNDS :7 \} ; Minor frame is out of Buf bounds

; second word is end of minor frame offset

; Define other miscellaneous constants

$$
\begin{array}{ll}
\text { SLIPR } & =100 \\
\text { LBUF } & =8192 \quad ; \text { Length of double buffer in } 16 \text { bit words } \\
\text { LIMBF } & =- \text { LBUF/2-SLIPR }
\end{array}
$$

$$
C_{-}=\{\$
$$

SLIPR: SLIPR, $\$ ;+/$ - range allowed for slip checking

LIMBF: LIMBF, $\$ \quad ;=-4196$, limiting address in search for

$$
\text { ; DCS synch }
$$

LIMB2: $-4095+16, \$ ;=-4079$, Length of scan to find second

LOKC9: $-4096, \$$

$$
\text { ; sync flag. }
$$

TTMSK: $8192, \$$; Mask for tick tock bit $(6.4 \mathrm{~kb}$ clock) ; 0010000000000000

TTERR: $-16384, \$$; Fill word in case of ticktock failure ; 1100000000000000

; BARKER CODE 24: 111110101111001100100000

PTRNH: -1293,\$ ; 1111101011110011

PTRNL: 8192$\} \quad ; 0010000000000000$

; Define various STATIC variables

$$
\mathrm{V}_{-}=\{\$
$$

SLIP :-64,\$ ; Offset required to give earlier minor frame ; this value requires that TTSAV be initialized ; to 0

TTSAV:0,\$ ; Storage for 'last' ticktock value

TTCNT:0,\$ ; Counter for ticktock failures

INITF:0,\$ ; Init/reinit flag, zero means init

TTTRY:0,\$ ; Try counter for ticktock phasing TTINI:0 \}

turn

endif

\section{Leftover stuff from HBRSYNC.SR}

FAZEF $=0$

BANANA $=0$; Set so that slip will not be reset on synch fail ; on $3 / 31$

; Initialize/define output arguments 
if N_ELEMENTS (MJF) eq 0 then MJF $=0 \mathrm{~L}$

$$
\begin{aligned}
& \text { HBR }=\text { intarr(4096) } \\
& \text { NRZ }=\text { intarr(128) } \\
& \text { Flags }=\text { intarr(3) }
\end{aligned}
$$

$V_{\text {_.TTTRY }}=0 \quad$; Set try count for ticktock phasing

SLIPS $=V_{-}$.SLIP ; Save beginning value for loop test

;

; Search for the first word in the buffer in which the frame synch word is set. this means that the DCS ground station is locked on and has found the minor frame synch word.

$$
\begin{aligned}
\text { bitsets }=\left(B U F\left(C_{-} \text {.SLIPR:8191) and } 2^{\wedge} B_{\_} \text {.FSYN }\right)\right. & ; \text { Bump C_.SLIPR words } \\
& ; \text { ahead to avoid } \\
& ; \text { early synch pattern }
\end{aligned}
$$

here0 = WHERE ( bitsets eq 0 , nzero ) ; The first word where

; the B_.FSYN bit is NOT

; set points to the first

; DCS frame synch

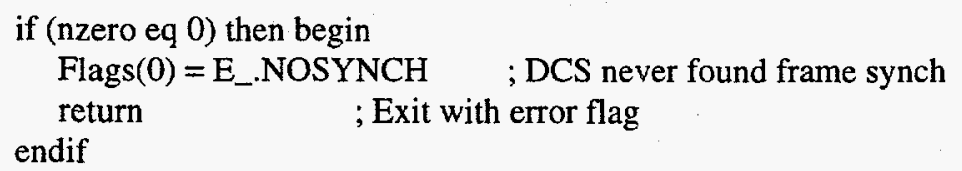

; Check next synch transition to see if it is 4096 words away.

; For each minor frame (i.e. $320 \mathrm{~ms}$ time interval), there are 16 sequential

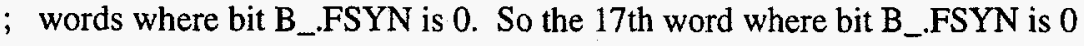

; should contain the next synch transition.

if (nzero gt 16) then begin ; If nzero eq 16, then next ; synch transition is out of buffer.

; Assume it's okay.

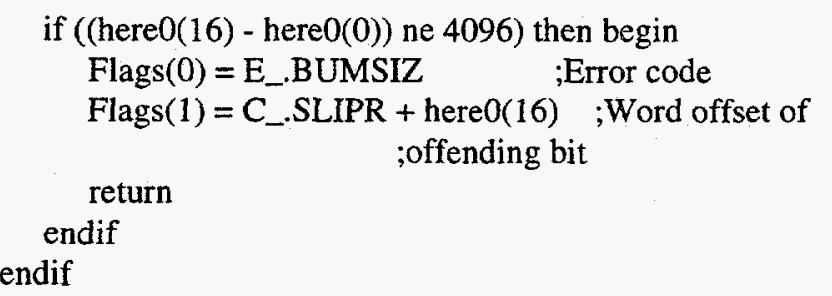

RENTR: $\quad$; Possible reentry if SLIP value is bad

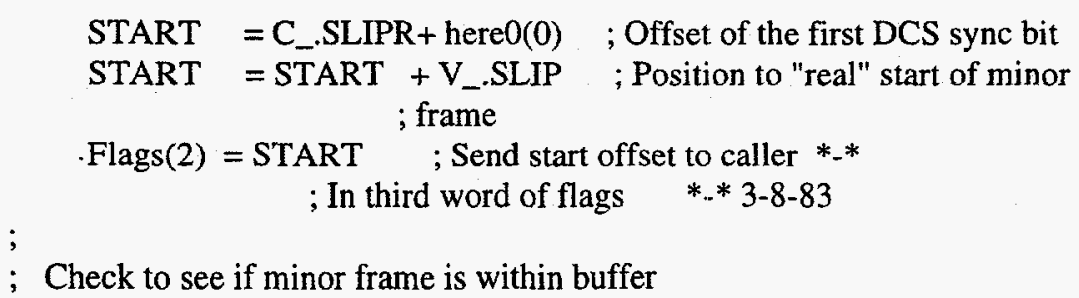




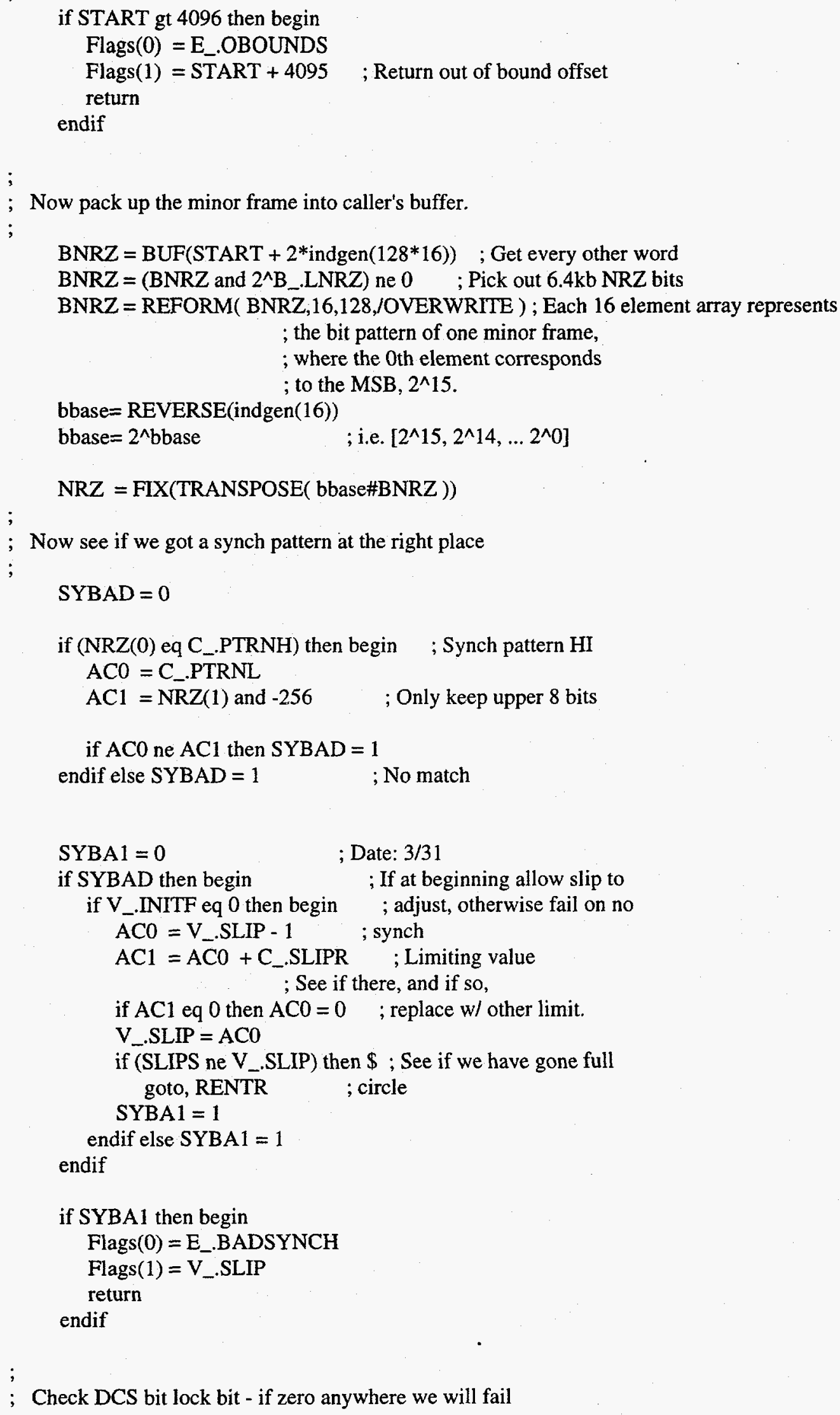




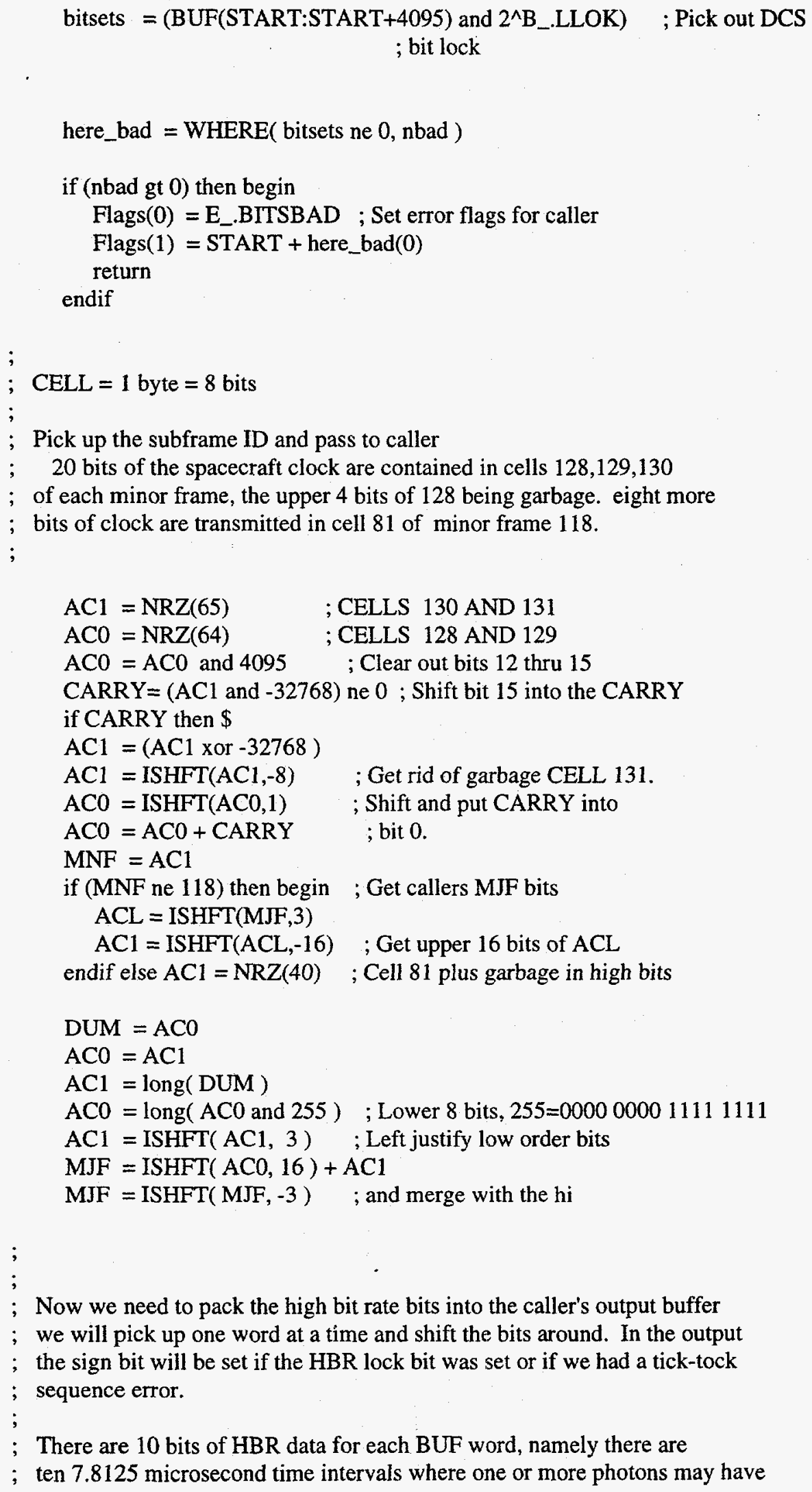


; been detected. Each bit that is set represents a detection of one or

; more photons. (We are assuming that the data is in the HBRB or High Bit

; Rate BIT format.)

;

$$
\begin{array}{cc}
\text { HBRwrds }=(B U F \text { and 63) } & \text { or } \$ ; \text { Bits } 0-5 \\
\text { (ISHFT(BUF,-2) and } 960) & \text { or } \$ ; \text { Bits } 8-11 \\
\text { (ISHFT(BUF and } 2^{\wedge} B_{-} . H L O K, 15-B_{-} \text {.HLOK)) }
\end{array}
$$

HBM_0:

V_.TTSAV $=$ V_.TTINI ; Value varies with assembly parameter "FAZEF"

INC1 $=0-1 \quad$; Set auto incrementer for destination buffer

INCO $=$ START $-1 ;$; Set auto incrementer for source buffer

COUNT $=4096 \quad$; Set number of words to move

;

; The bit picked up by TTMSK should be "tick-tock"ing between values

; 0 and 1 for sequential BUF words.

for $i=1$, COUNT do begin

$\mathrm{INC} 0=\mathrm{INC} 0+1$

$\mathrm{AC1}=\mathrm{BUF}(\mathrm{INC} 0) \quad ;$ Load up next source word

$\mathrm{AC} 2=\mathrm{AC1}$ and $\mathrm{C}_{-}$.TTMSK ; Pick up NRZ clock bit only

$\mathrm{ACO}=\mathrm{V}_{-} \cdot \mathrm{TTSAV}$

$V_{-}$.TTSAV $=A C 2$

HBRfill $=$ HBRwrds $(\mathrm{INC} 0)$

if ( $\mathrm{AC} 2$ eq $\mathrm{AC} 0)$ then begin ; If next "tick" is a "tock"-> error $V_{-}$.TTSAV $=C_{-}$.TTMSK xor AC2

$\mathrm{ACO}=\mathrm{C}_{-}$.TTERR

$\mathrm{INCO}=\mathrm{INC0}-1 \quad ;$ Move back in the source

if $\mathrm{INC} 0$ ne 0 then $\mathrm{HBRfill}=\mathrm{ACO}$; and go store the fill data

endif

$\mathrm{INC} 1=\mathrm{INCl}+1$

$\operatorname{HBR}(\operatorname{INC} 1)=$ HBRfill

endfor

;

; Now calculate the number of words actually loaded from the buffer. should be 4096 - if not we had a ticktock error.

$\mathrm{ACO}=\mathrm{START}+4095$

$\mathrm{ACO}=\mathrm{ACO}-\mathrm{INCO}$

if $\mathrm{ACO}$ ne 0 then begin ; Will be zero if no tick tock errors

if (V_.TTTRY ne 0$)$ or $\$$; Have tried both phases - just fail

(V_.INITF ne 0 ) then $\$$; See if we ever had a good one

begin ; yes so fail now

Flags $(0)=E_{\_}$.TIKTOK $\quad ;$ Return with error

Flags $(1)=\mathrm{AC} 0$

return

endif 


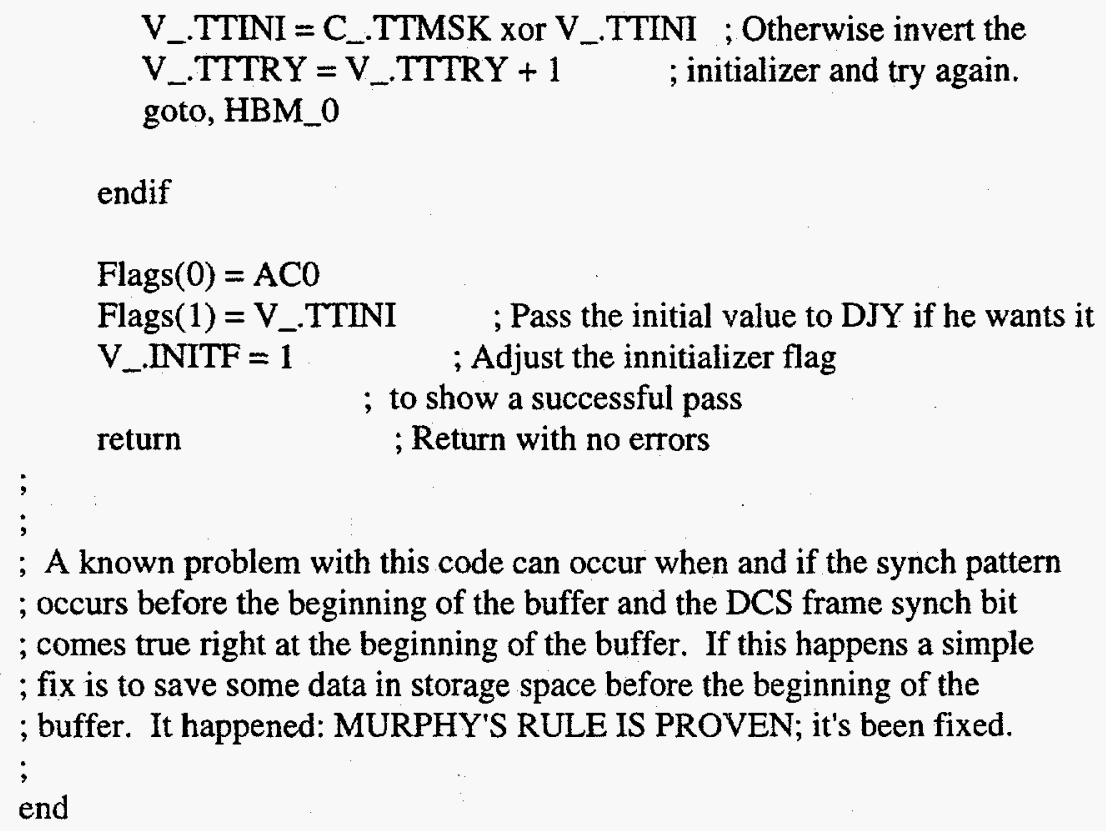

\section{A.4.2 Photon Time Interval Format}

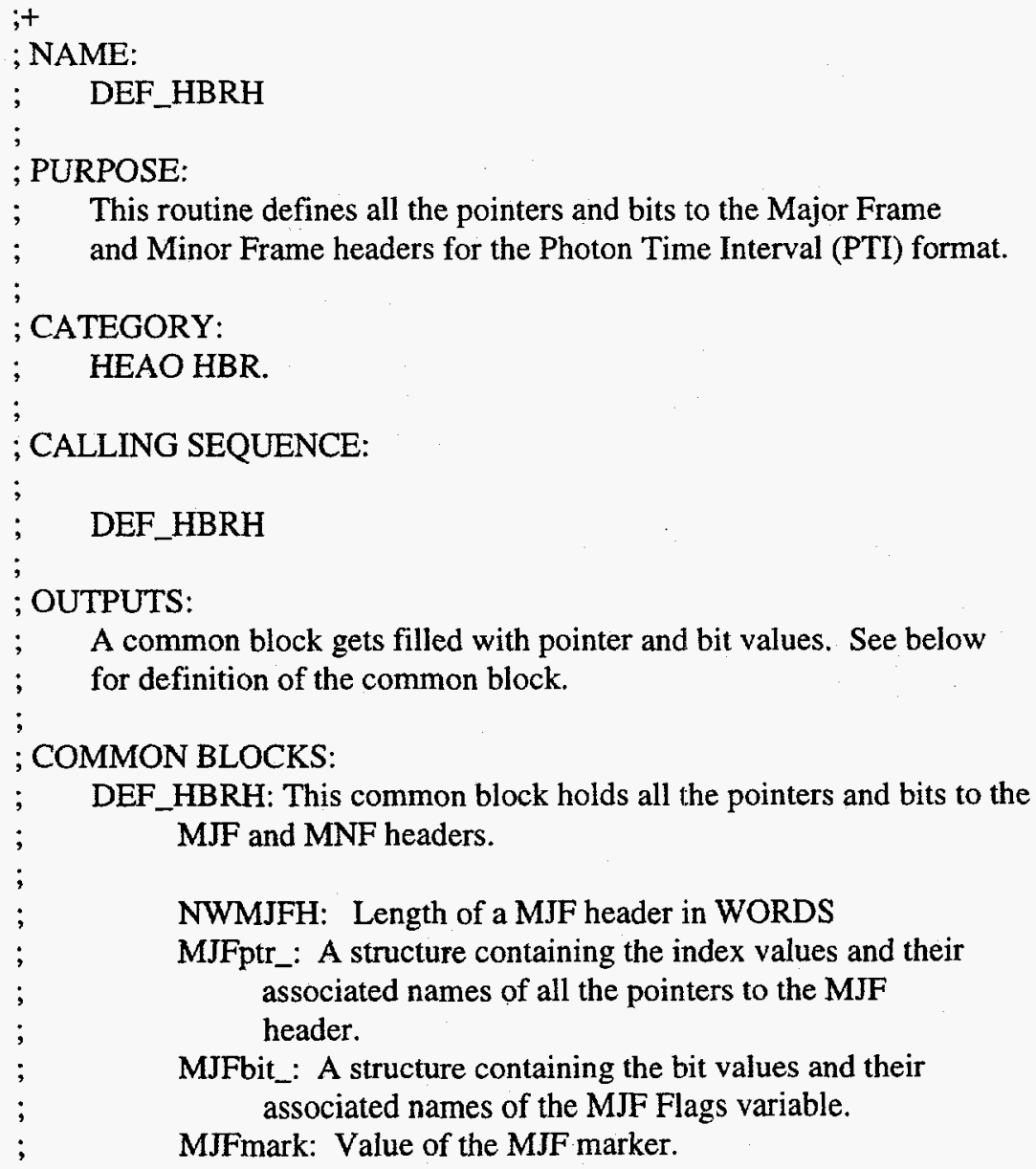


NWMNFH: Length of a MNF header in WORDS

MNFptr_: A structure containing the index values and their associated names of all the pointers to the MNF header.

MNFbit_: A structure containing the bit values and their associated names of the MNF Flags variable.

MNFmark: Value of the MNF marker.

(*Note: For a description of each pointer or bit, see DEF_HBRH.pro)

; MODIFICATION HISTORY:

; Written by: Han Wen, July 1995.

;-

pro DEF_HBRH

common DEF_HBRH, NWMJFH, MJFptr_, MJFbit_, MJFmark, \$

NWMNFH, MNFptr_, MNFbit_, MNFmark, \$

NWNRZB, NWPTIR

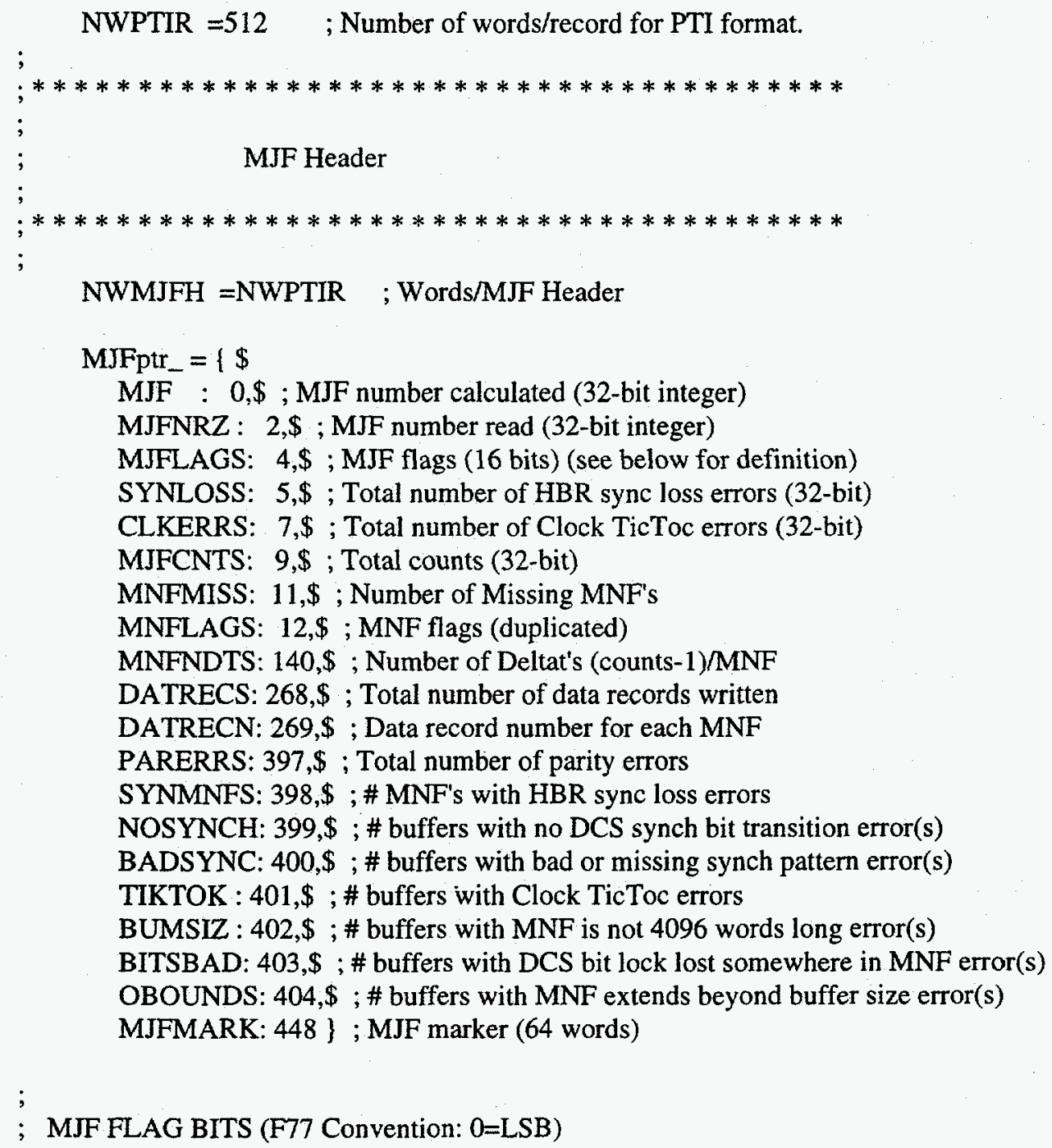




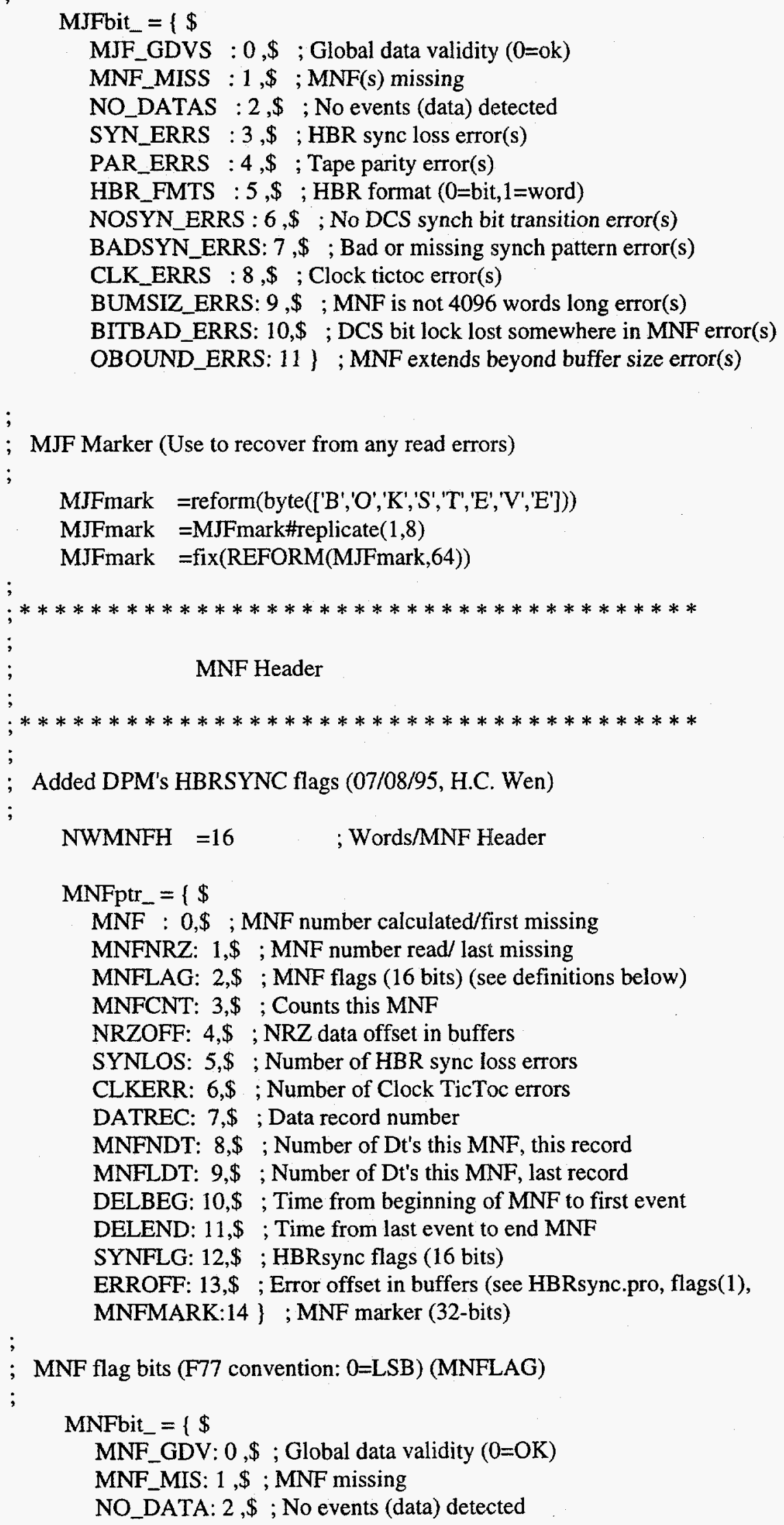


SYN_ERR: $3, \$$; HBR sync loss error(s)

CLK_ERR: $4, \$$; Clock tictoc error(s)

PAR_ER1: $5, \$$; Tape parity error buffer 1

PAR_ER2: $6, \$$; Tape parity error buffer 2

SUSPECT: 7 \} ; Current or previous buffer(s) have one or

$$
\text { ; more fatal HBRSYNC errors. }
$$

$: 8, \$ ;$ Unassigned

$: 9, \$ ;$ Unassigned

$: 10, \$$; Unassigned

$: 11, \$$; Unassigned

$: 12, \$$; Unassigned

$: 13, \$ ;$ Unassigned

$: 14, \$ ;$ Unassigned

$: 15\}$; Unassigned

;

MNF Marker (Use to recover from any read errors)

MNFmark $=142857143 \mathrm{~L}$

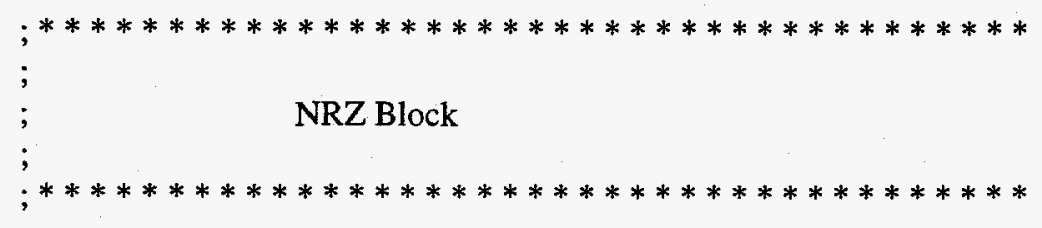

end

NWNRZB $=128 * 128 ;$ Words/NRZ block (128 words/MNF)

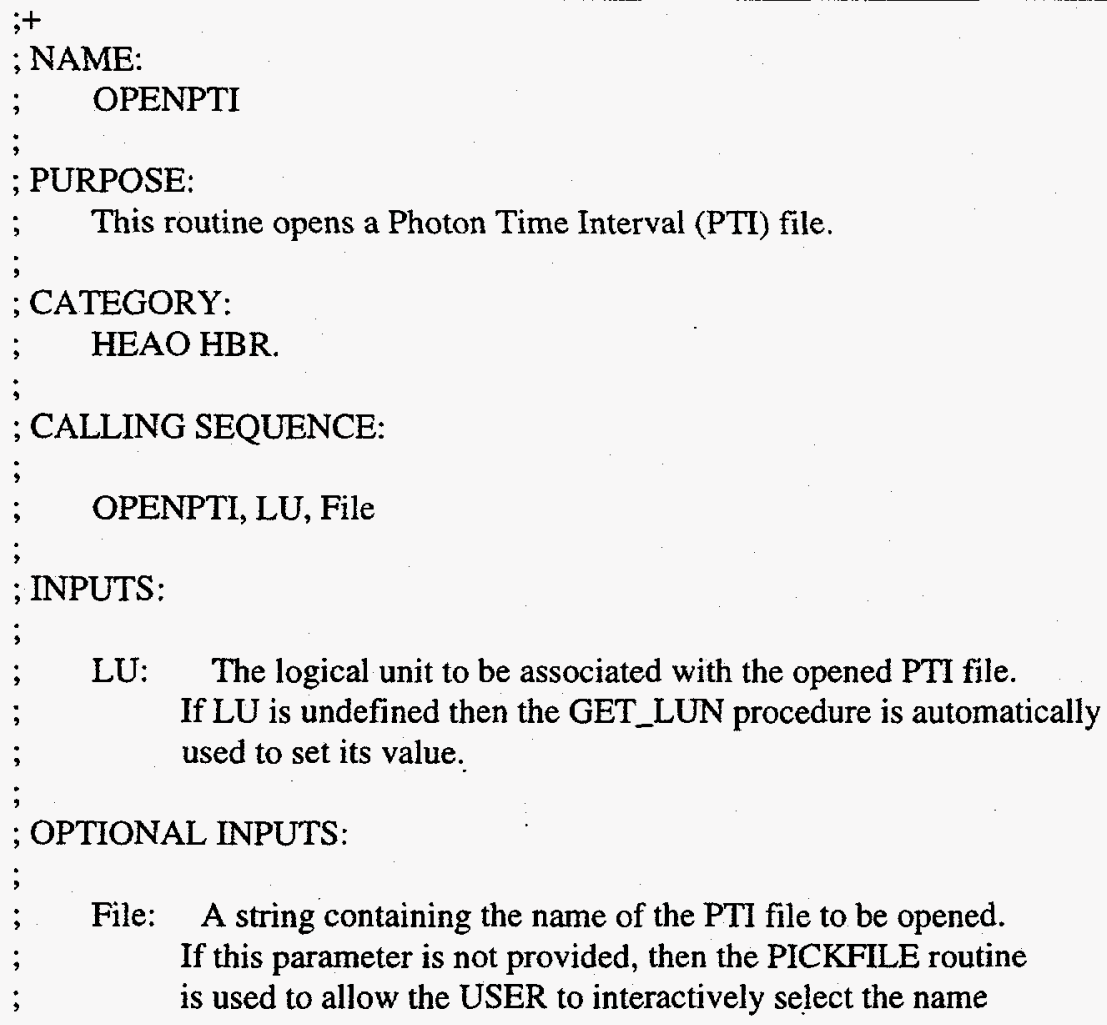

This routine opens a Photon Time Interval (PTI) file.

; CATEGORY:

; HEAO HBR.

; CALLING SEQUENCE:

;

; OPENPTI, LU, File

;

; INPUTS:

; LU: The logical unit to be associated with the opened PTI file.

; If LU is undefined then the GET_LUN procedure is automatically

; used to set its value.

;

; OPTIONAL INPUTS:

; File: A string containing the name of the PTI file to be opened.

; If this parameter is not provided, then the PICKFILE routine

; is used to allow the USER to interactively select the name

; 


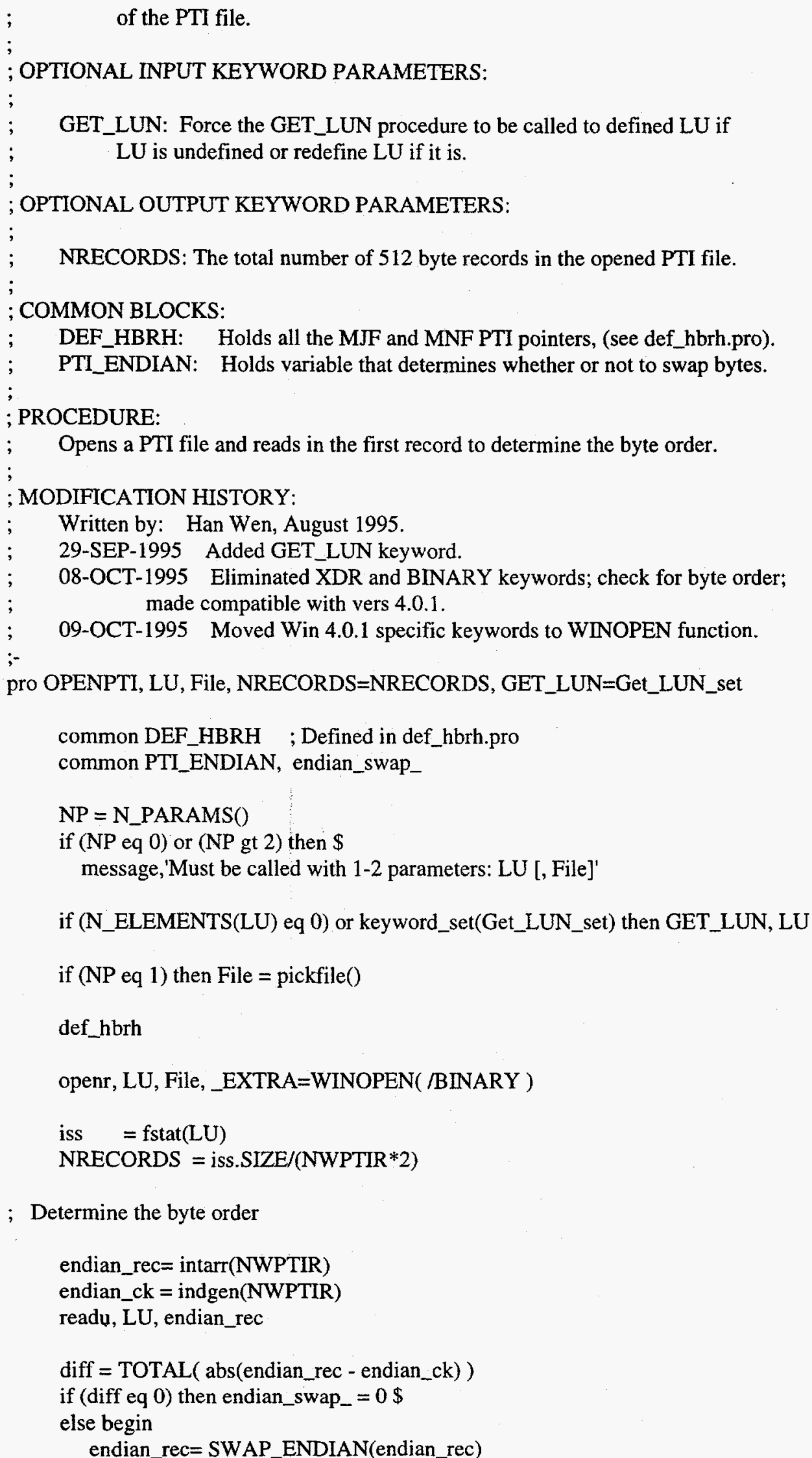




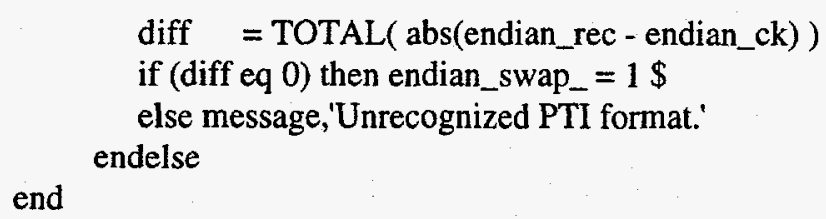

This routine reads in the next MJF block of Photon Time Interval (PTI)

; file and supplies it to the USER in a structure variable.

; CATEGORY:

; HEAO HBR.

;

; CALLING SEQUENCE:

;

; $\quad$ READPTI, LU, Block

;

; INPUTS:

LU: Logical unit associated with the current PTI file.

; OUTPUTS:

Block: Structure variable holding the next MJF block of PTI data.

;he tags are defined as follows:

; $\quad$ MJFH: An array holding the MJF header, intarr(512).

MNFH: An array holding the MNF header for each MNF present in this MJF block, intarr(16,nMNFH*).

NRZ: An array holding the NRZ data**, intarr(128,nMNF*).

PTI: An array holding all the photon time intervals** in 7.8125 usec bins in this MJF block, lonarr(ndt).

(E.g. A value of 1 means that two photons occurred within adjacent 7.8125 usec bins.)

* (see PROCEDURE below for definitions).

**(see PROCEDURE below for caveats).

; OUTPUT KEYWORD PARAMETERS:

ERROR: Error status code:

0 , Success.

1, End of File.

2, Could not find MJF marker.

3, Cound not find MNF marker.

!error, I/O error.

; COMMON BLOCKS:

; DEF_HBRH: Holds all the MJF and MNF PTI pointers, (see def_hbrh.pro).

;

; PROCEDURE: 


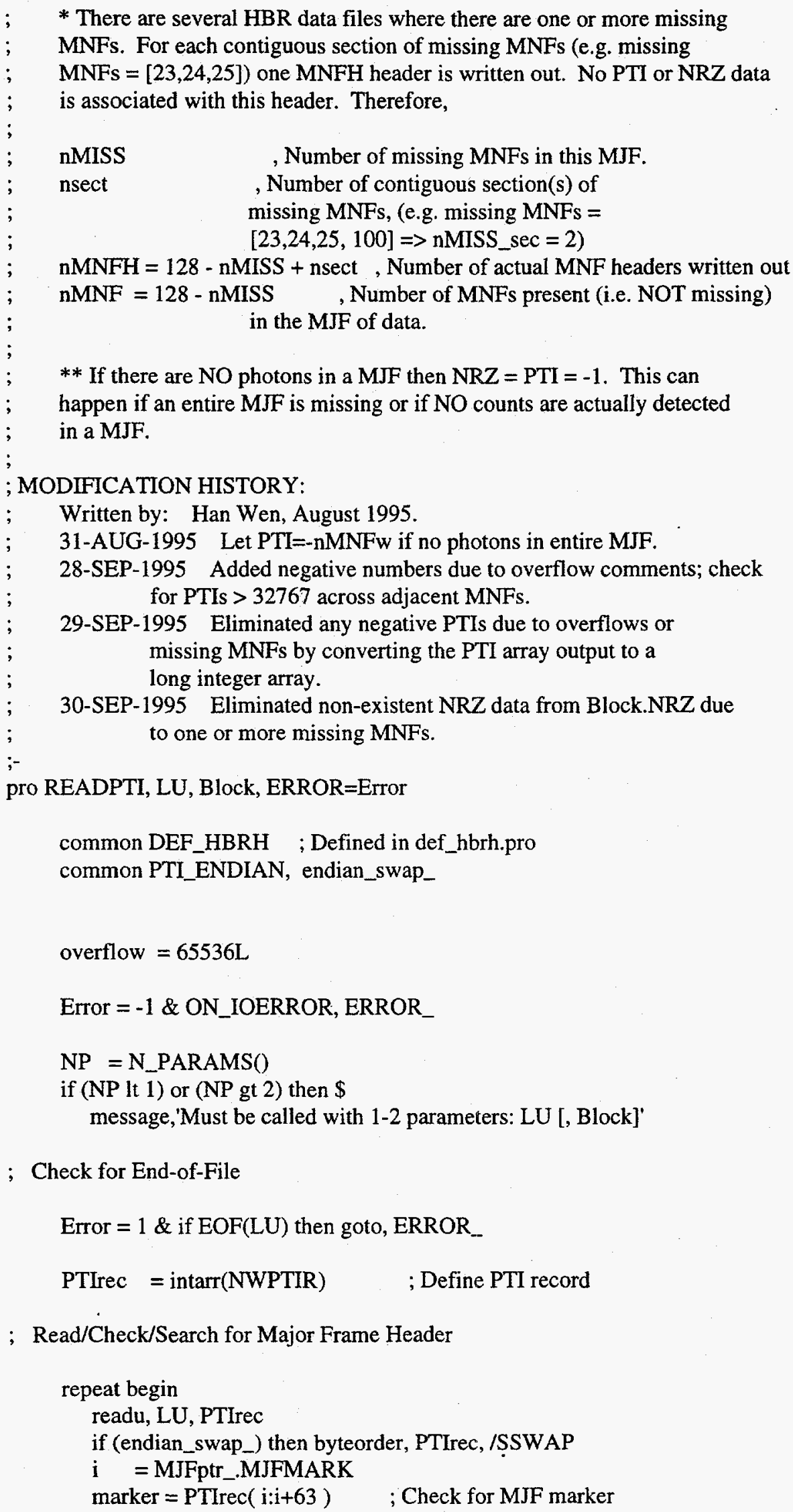




\footnotetext{
diff $=$ TOTAL (abs(marker - MJFmark))

endrep until (diff eq 0 ) or $\operatorname{EOF(LU)~}$

Error $=2$ \& if EOF(LU) then goto, ERROR_

$\mathrm{MJFH} \quad=$ PTIrec
}

; Read in NRZ (6.4 kps) Data block

$$
\begin{aligned}
& \text { NRZ = intarr(NWNRZB) ; NRZ block } \\
& \mathrm{nMNF}=128 \text {; Number of Minor frames/MJF } \\
& \text { NWNRZ }=\text { NWNRZB/nMNF } \quad ;=128 \text { words of NRZ data/MNF }
\end{aligned}
$$

; Read in all Minor Frame data records

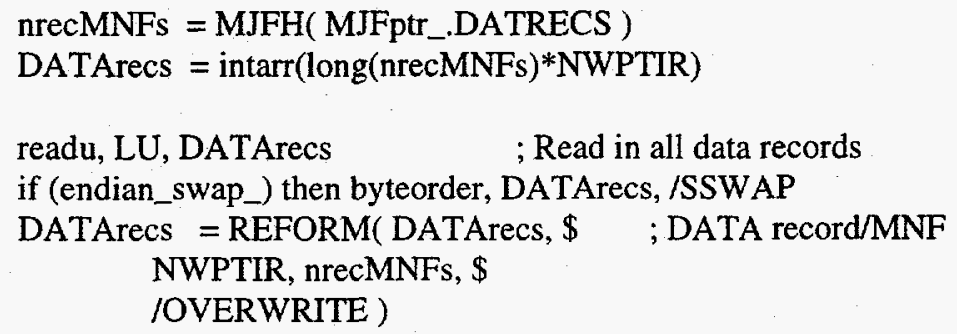

; Determine MNF record numbers

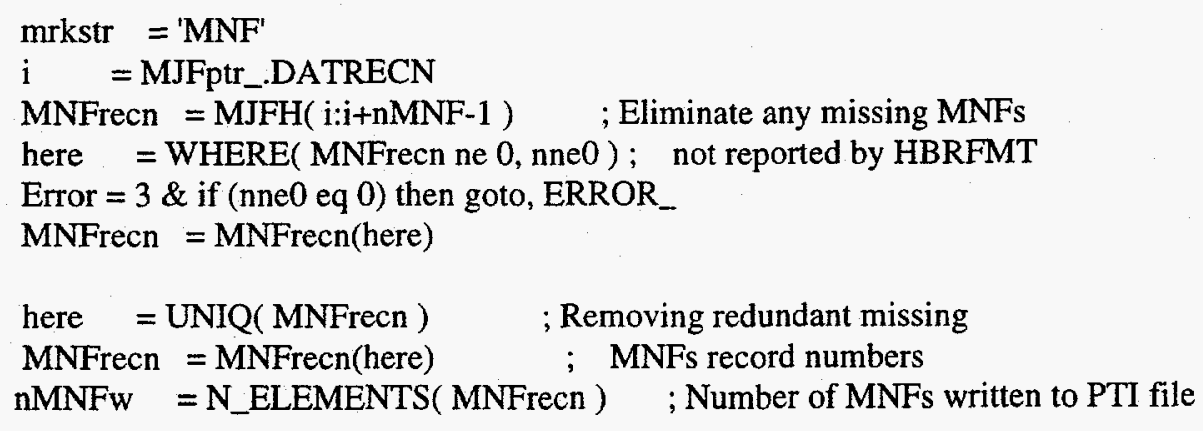

; Extract MNF headers

rec_offset $=$ MNFrecn - MNFrecn $(0)$

MNFH = DATArecs( 0:NWMNFH-1, rec_offset )

; Check MNF marker

i $\quad=$ MNFptr_.MNFMARK

markint $=$ MNFH $\left(\mathrm{i}: \mathrm{i}+1,{ }^{*}\right)$

markers $=$ lonarr ( $\mathrm{nMNFw}$ )

EQUIV, markers, markint 
here = WHERE (markers ne MNFMARK, nne0 )

Error $=3 \&$ if $($ nne0 gt 0$)$ then goto, ERROR

; Extract number of DATA record numbers written for each MNF

if (nMNFw eq 1$)$ then nrecDATA = nrecMNFs - rec_offset $(0) \$$

else begin

nrecDATA $=$ [rec_offset $(1: \mathrm{nMNFw}-1)$, nrecMNFs]

nrecDATA $=$ nrecDATA - rec_offset $(0: n M N F w-1)$

endelse

; and the Photon Time Intervals

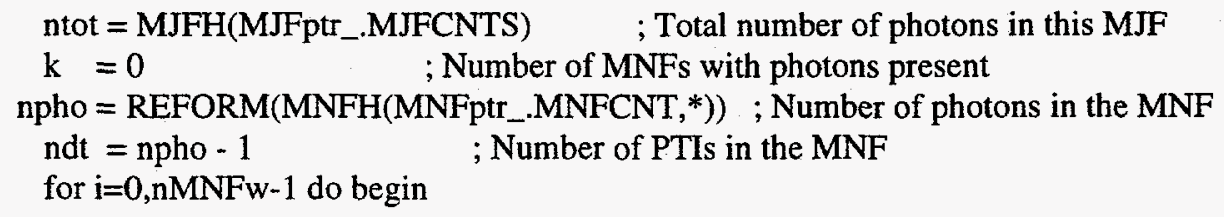

; See if this is a MISSING MNF block

MNFmiss_bit $=$ MNFH(MNFptr_.MNFLAG,i) and 2^MNFbit_.MNF_MIS

if (MNFmiss_bit ne 0) then goto, SKIP_MISS

case 1 of

; Two or more photons in MNF

(npho(i) gt 1): begin

$\mathrm{k}=\mathrm{k}+1$

nrec $=$ nrecDATA $(\mathrm{i})$

$\mathrm{j}=$ rec_offset $(\mathrm{i})$

data $=$ DATArecs $(*, \mathrm{j}: \mathrm{j}+$ nrec-1 $)$

data $=$ REFORM (data, nrec*NWPTIR, /OVERWRITE )

data $=$ long $($ data $($ NWMNFH: NWMNFH $+\operatorname{ndt}(\mathbf{i})-1))$

; Check for 16-bit overflows

hovr $=$ where (data lt 0 , novr $)$

if (novr gt 0 ) then $\$$

data(hovr) $=$ data(hovr) + overflow

if $(\mathrm{k}$ eq 1$)$ then PTI = data $\$$

else begin

; Extract time interval from beginning of MNF

; to 1st photon. With dt_EOMNF, construct the

; time interval between last and first photons

; of "adjacent" MNFs.

dt_SOMNF $=$ long(MNFH( MNFptr_.DELBEG,i $)$ )

if $\left(\mathrm{dt} \_S O M N F\right.$ it 0$)$ then dt_SOMNF $=\mathrm{dt} \_$SOMNF + overflow

dt_pho1 $=d t \_E O M N F+d t \_S O M N F$

; If there are missing MNFs between current and

; previous MNFs, then we must add that time interval 
; to dt_pho1

mnf_skip $=$ MNFH(MNFptr_.MNFNRZ,i $)-\$$

MNFH(MNFptr_.MNFNRZ,i_last) - 1

if (mnf_skip gt 0 ) then $\$$

dt_pho1 = dt_pho1 + mnf_skip*40960L

; Finally add this 1 st and remaining time intervals for

; this MNF to the PTI array.

PTI $=[$ PTI, dt_pho1, data $]$

endelse

; Extract time interval from last photon

; to end of MNF

dt_EOMNF $=$ long $($ MNFH $($ MNFptr_.DELEND,i $))$

if (dt_EOMNF lt 0 ) then dt_EOMNF = dt_EOMNF + overflow

i_last $=\mathrm{i}$

end

; One photon in MNF

(npho(i) eq 1) : begin

$\mathrm{k}=\mathrm{k}+1$

if $(\mathrm{kgt} 1)$ then begin

dt_SOMNF $=$ long(MNFH( MNFptr_.DELBEG,i $))$

if (dt_SOMNF It 0 ) then dt_SOMNF = dt_SOMNF + overflow $\mathrm{dt} \_$pho1 $=\mathrm{dt} \_$EOMNF $+\mathrm{dt} \_S O M N F$

PTI $=[$ PTI,dt_pho1 $]$

endif

dt_EOMNF $=$ long(MNFH $($ MNFptr_.DELEND, $\mathbf{i}))$

if $\left(\mathrm{dt} \_\right.$EOMNF $\left.1 \mathrm{t} 0\right)$ then dt_EOMNF $=\mathrm{dt} \_$EOMNF + overflow

end

; NO photons in MNF

(npho(i) eq 0) : if $(\mathrm{k}$ gt 1$)$ then dt_EOMNF $=\mathrm{dt} \_$EOMNF $+40960 \mathrm{~L}$

SKIP_MISS:

endcase

endfor

if (N_ELEMENTS(PTI) eq 0) then PTI=-1

; MNF headers describing missing MNFs have NO associated NRZ data.

; The actual amount of NRZ data contained in a $(128,128)$ NRZ block is

; (128,nMNF-nskip), where nskip is the number of MNF headers with

; missing MNFs.

b_MNFmiss $=$ MNFH(mnfptr_.MNFLAG, $\left.{ }^{*}\right)$ and $2^{\wedge}$ MNFbit_.MNF_MIS

hdata $=$ where (b_MNFmiss eq 0,ndata) ;i.e. ndata $=$ nMNF-nskip

if (ndata gt 0 ) then $\mathrm{NRZ}=\mathrm{NRZ}\left({ }^{*}, 0\right.$ :ndata- 1$) \$$

else NRZ $=-1$

block $=\{\mathrm{MJFH}: \mathrm{MJFH}, \$$ 


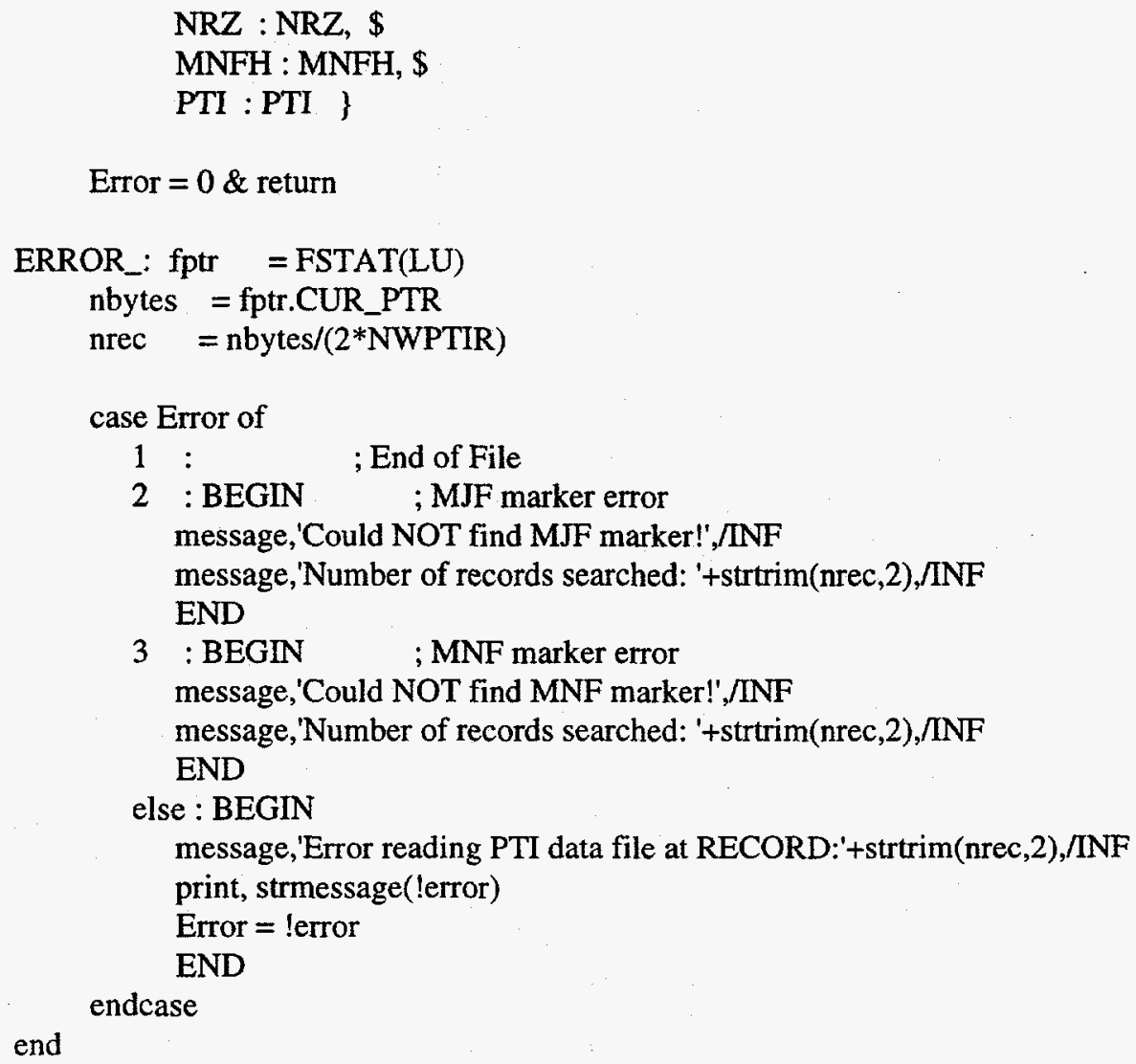




\section{A.4.3 Concatenated Time Interval Format}

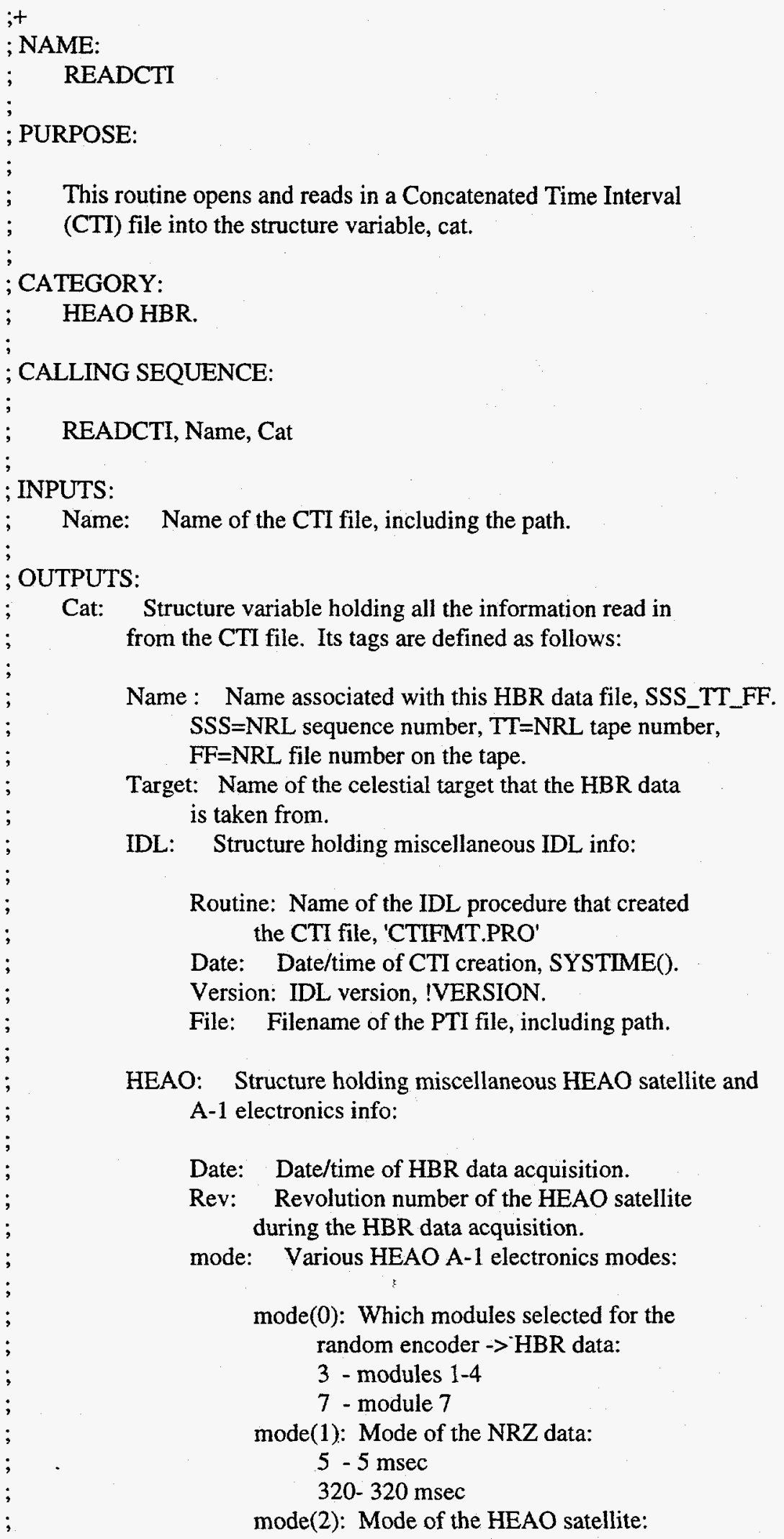




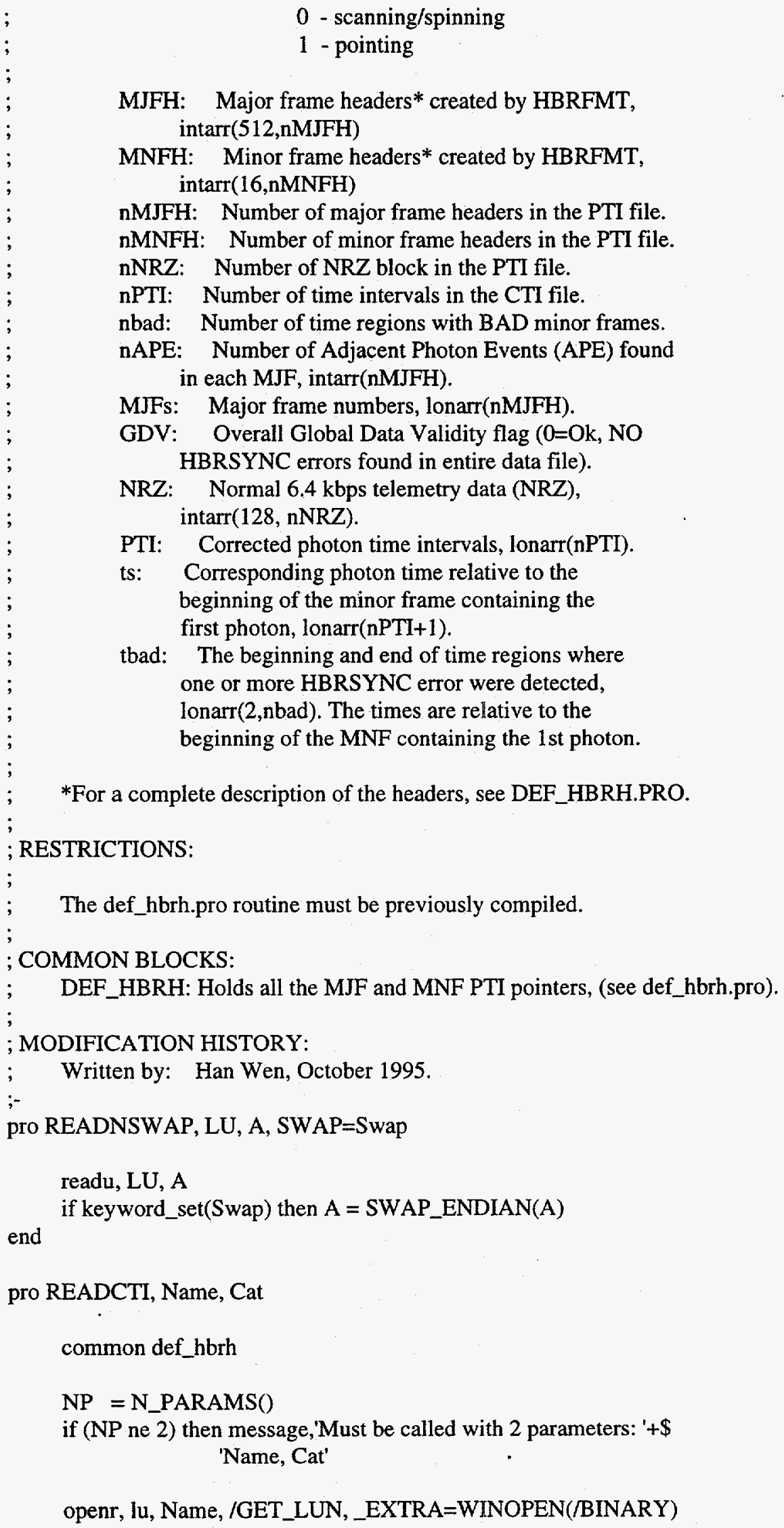


; Determine the byte order

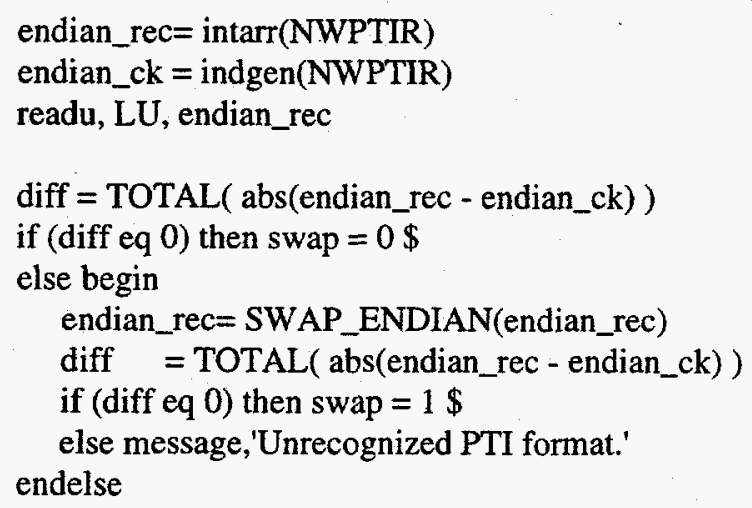

; Read in the string headers

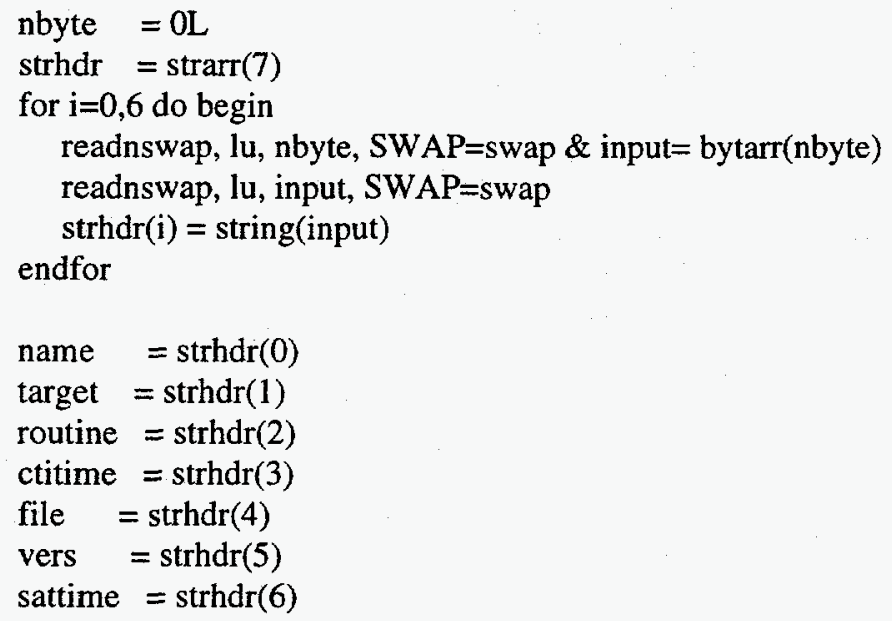

; Read in miscellaneous HEAO info

readnswap, lu, nbyte, SWAP=swap \& rev $=0$

readnswap, lu, rev, SWAP=swap

readnswap, lu, nbyte, SWAP=swap \& mode $=$ intarr(nbyte/2)

readnswap, lu, mode, SWAP=swap

; Extract the MJF and MNF headers

readnswap, lu, nbyte, SWAP=swap \& nMJF = nbyte/4 \& MJFs = lonarr(nMJF)

readnswap, lu, MJFs, SWAP=swap

readnswap, lu, nbyte, SWAP=swap \& MJFH = intarr(512,nMJF)

readnswap, lu, MJFH, SWAP=swap

readnswap, lu, nbyte, SWAP $=$ swap \& $\mathrm{nMNF}=$ nbyte $/ 32 \& \mathrm{MNFH}=\operatorname{intarr}(16, \mathrm{nMNF})$

readnswap, lu, MNFH, SWAP=swap

readnswap, lu, nbyte, SWAP=swap \& GDV $=0$

readnswap, lu, GDV, SWAP=swap

; Get the 6.4 kbps data and the photon info

readnswap, lu, nbyte, SWAP=swap \& nNRZ = nbyte $256 \& N R Z=\operatorname{intarr}(128, n N R Z)$ 
readnswap, lu, NRZ, SWAP=swap

readnswap, lu, nbyte, $\mathrm{SWAP}=$ swap $\& \mathrm{nPTI}=$ nbyte $/ 4 \quad \& \mathrm{PTI}=\operatorname{lonarr}(\mathrm{nPTI})$

readnswap, lu, PTI, SWAP=swap

readnswap, lu, nbyte, SWAP=swap \& nts = nbyte 4 \& ts = lonarr(nts)

readnswap, lu, ts, SWAP=swap

readnswap, lu, nbyte, SWAP=swap \& nbad $=$ nbyte $/ 8 \&$ tbad $=$ lonarr $(2$, nbad $)$

readnswap, lu, tbad, SWAP=swap

readnswap, lu, nbyte, SWAP=swap \& nAPE = intarr(nbyte/2)

readnswap, lu, nAPE, SWAP=swap

if (nPTI eq 1) and (PTI(0) eq -1) then $\mathrm{nPTI}=0 \mathrm{~L}$

if (nbad eq 1 ) and ( $\operatorname{tbad}(0)$ eq -1$)$ then $\mathrm{nbad}=0 \mathrm{~L}$

free_lun,lu

; Pack the IDL structure variables

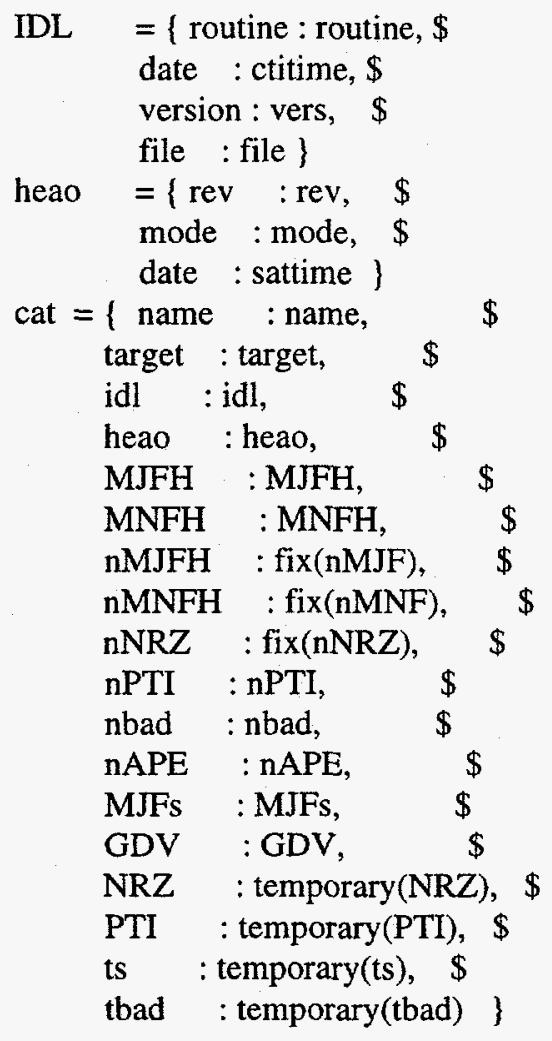

end 


\section{A.4.4 NRZ Format}

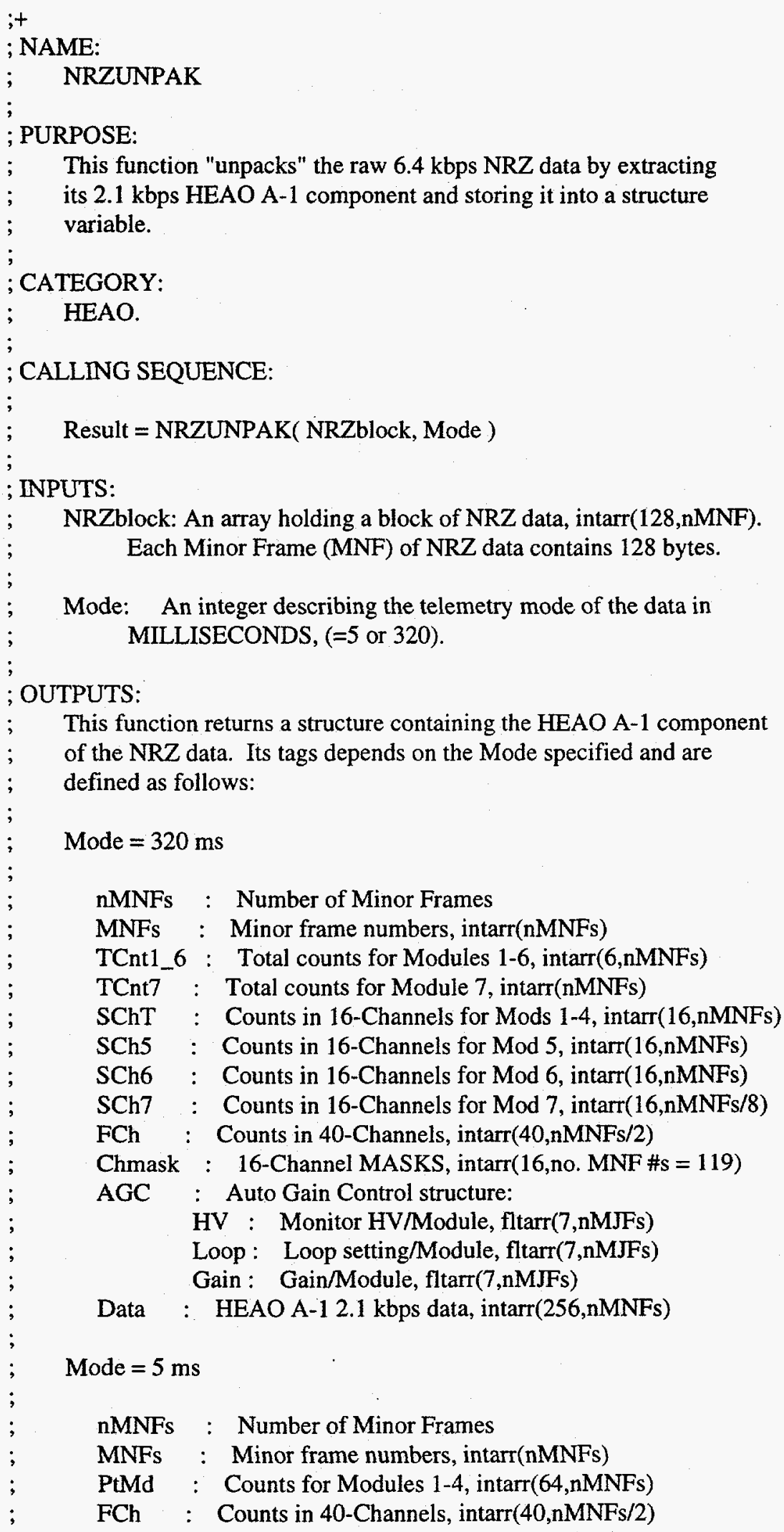

This function "unpacks" the raw $6.4 \mathrm{kbps}$ NRZ data by extracting its $2.1 \mathrm{kbps}$ HEAO A-1 component and storing it into a structure

This function returns a structure containing the HEAO A-1 component of the NRZ data. Its tags depends on the Mode specified and are defined as follows:

Mode $=320 \mathrm{~ms}$

nMNFs : Number of Minor Frames

MNFs : Minor frame numbers, intarr(nMNFs)

TCnt1_6 : Total counts for Modules 1-6, intarr(6,nMNFs)

TCnt7 $^{-}$: Total counts for Module 7, intarr(nMNFs)

SChT : Counts in 16-Channels for Mods 1-4, intarr(16,nMNFs)

SCh5 : Counts in 16-Channels for Mod 5, intarr(16,nMNFs)

SCh6 : Counts in 16-Channels for Mod 6, intarr(16,nMNFs)

$\mathrm{SCh} 7$ : Counts in 16-Channels for Mod 7, intarr(16,nMNFs/8)

FCh : Counts in 40-Channels, intarr(40,nMNFs/2)

Chmask : 16-Channel MASKS, intarr(16,no. MNF \#s = 119)

AGC : Auto Gain Control structure:

HV : Monitor HV/Module, fltarr(7,nMJFs)

Loop : Loop setting/Module, fltarr(7,nMJFs)

Gain : Gain/Module, fltarr(7,nMJFs)

Data : HEAO A-1 2.1 kbps data, intarr(256,nMNFs)

Mode $=5 \mathrm{~ms}$

nMNFs : Number of Minor Frames

MNFs : Minor frame numbers, intarr(nMNFs)

PtMd : Counts for Modules 1-4, intarr(64,nMNFs)

FCh : Counts in 40-Channels, intarr(40,nMNFs/2) 


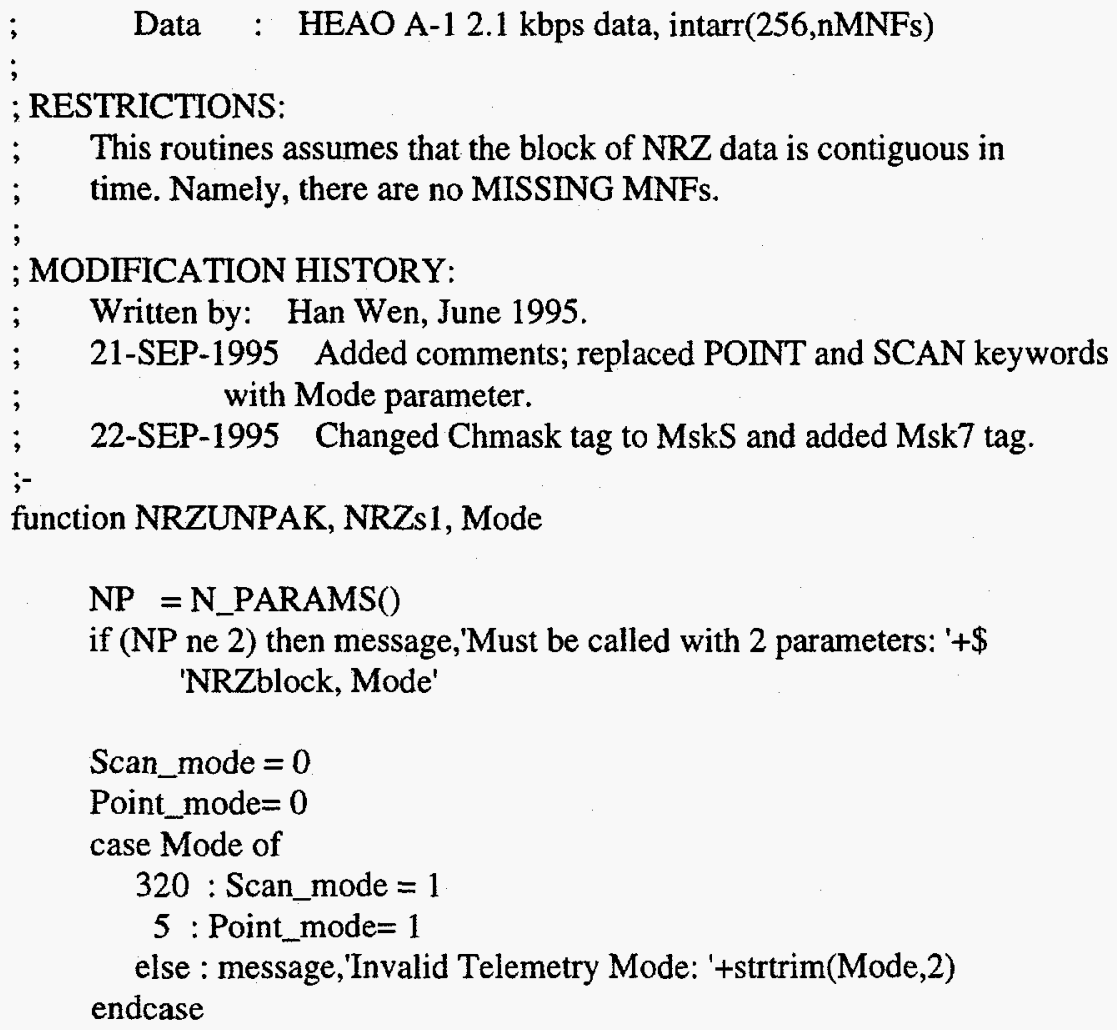

; Define all BYTE offsets to all HEAO A-1 NRZ Data

; Scan Mode Telemetry Format

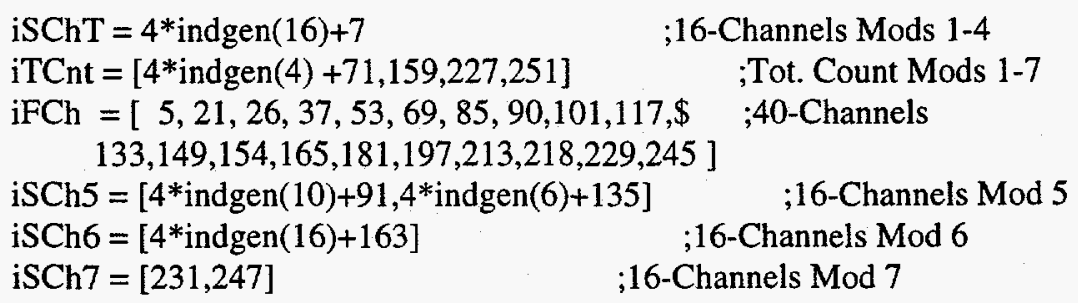

; Point Mode Telemetry Format

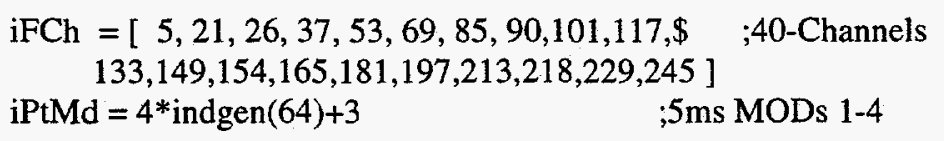

if keyword_set( Scan_mode ) then Sarr = [iSChT, iTCnt, iFCh, iSCh5, iSCh6, iSCh7 ]

if keyword_set( Point_mode) then Sarr $=[\mathrm{iFCh}, \mathrm{iPtMd}]$

Type = strarr(256)

Type(Sarr)= 'S'

; Convert to byte array

$$
\begin{aligned}
& \text { nMNFs }=\text { n_elements(NRZs1)/128 } \\
& \text { NRZs }=\text { REFORM(NRZs1, nMNFs*128) } \\
& \mathrm{i}=0
\end{aligned}
$$


j $\quad=2 *$ indgen(128)

$\mathrm{k} \quad \mathrm{j}+1$

$\mathrm{NRZb}=$ bytarr(256,nMNFs)

$\mathrm{NRZb}\left(\mathrm{j},{ }^{*}\right)=$ ISHFT(NRZs,-8) ;Upper 8 bits fill the "first" bytes

$\mathrm{NRZ} b\left(\mathrm{k},{ }^{*}\right)=\mathrm{NRZs}$ and $377^{\prime} \mathrm{O} \quad$;Lower 8 bits fill the "second" bytes

; Convert quasi-logarithmic numbers for Science data

Data $=$ fix $(N R Z b)$

here = where(Type eq 'S')

$\operatorname{Data}\left(\right.$ here,$\left.{ }^{*}\right)=\operatorname{ACNVRT}\left(\right.$ Data $\left(\right.$ here,$\left.\left.{ }^{*}\right)\right)$

; Extract MNF numbers (Taken from HBRSYNC)

$\mathrm{AC1}=\mathrm{REFORM}(\mathrm{NRZs} 1(65, *))$

; CELLS 130 AND 131

here $=$ WHERE $((A C 1$ and -32768$)$ ne $0, n C A R R Y) \quad$; Shift bit 15 into the CARRY

if (nCARRY gt 0) then AC1(here) = AC1(here) xor -32768

$\mathrm{AC} 1=\operatorname{ISHFT}(\mathrm{ACl},-8) \quad$; Get rid of garbage CELL 131.

$\mathrm{MNFs}=\mathrm{ACl}$

h0 = WHERE(MNFs eq MNFs(0),nMJFs)

; Extract NRZ depending on USER specified telemetry mode

i0 $=0$

if $(\operatorname{MNFs}(0)$ and 1$)$ eq 1 then $i 0=1 \quad$;Odd MNF

CASE 1 OF

keyword_set(Scan_mode): BEGIN

; A-1 Scan Telemetry Mode

; Extract the COUNTS

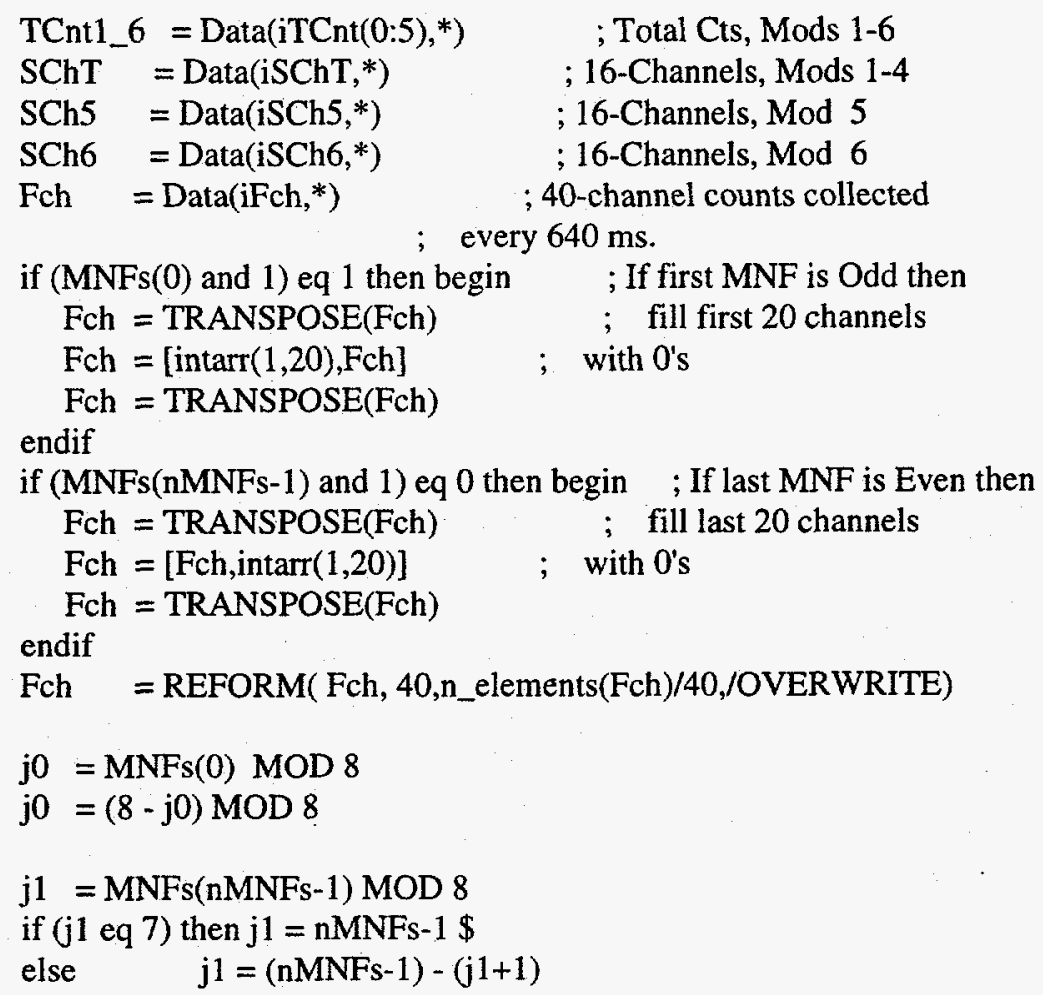




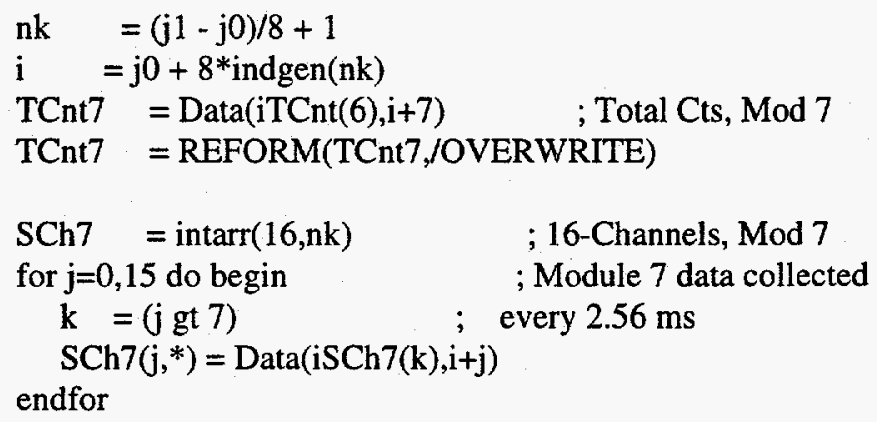

; See which energy channels are masked off for Modules 1-4

$$
\begin{aligned}
& \text { imsk_MNF }=119 \quad ; \text { MNF index } \\
& \text { imsk_word }=255 \quad ; \text { NRZ word index } \\
& \text { hmask = where(MNFs eq imsk_MNF) } \\
& \text { MSBs = Data(imsk_word,hmask) } \\
& \text { LSBs = Data(imsk_word,hmask+1) } \\
& \text { masks = ISHFT(MSBs, } 8)+ \text { LSBs } \\
& \begin{array}{l}
\text { MskS } \quad \text { intarr }\left(16, n \_\right. \text {elements(hmask)) } \\
\text { for } \left.\mathrm{i}=0,15 \text { do MskS(i, }{ }^{*}\right)=\left(\text { masks and } 2^{\wedge} \mathrm{i}\right) / 2^{\wedge} \mathrm{i}
\end{array}
\end{aligned}
$$

; See which energy channels are masked off for Module 7

$$
\begin{aligned}
& \text { imsk_MNF }=117 \quad ; \text { MNF index } \\
& \text { imsk_word }=255 \quad \text {; NRZ word index } \\
& \text { hmask = where(MNFs eq imsk_MNF) } \\
& \text { MSBs = Data(imsk_word,hmask) } \\
& \text { LSBs = Data(imsk_word,hmask+1) } \\
& \text { masks }=\text { ISHFT }(M S B s, 8)+\text { LSBs } \\
& \text { Msk7 = intarr(16,n_elements(hmask)) } \\
& \text { for } \mathrm{i}=0,15 \text { do } \operatorname{Msk} 7\left(\mathrm{i},{ }^{*}\right)=\left(\text { masks and } 2^{\wedge} \mathrm{i}\right) / 2^{\wedge} \mathrm{i}
\end{aligned}
$$

; See what the AGC MONITOR, LOOP and GAIN is for each module

$$
\begin{aligned}
& \text { iAGC_word }=[87,87,87,87,255,255,255] \quad ; \text { NRZ word index for } \\
& \text {; each module } \\
& \text { iHV_MNF }=[16,48,80,112,16,48,80] \quad ; \text { MNF index for } \\
& \text { iAGC_MNF }=[27,59,91,123,27,59,91] ; \text { each module } \\
& \text { iAGC_Lbit }=1 \text {; LOOP Bit index of } \\
& \text { iAGC_Gbit }=2 \quad \text {; GAIN Bit index of } \\
& \text {; the NRZ word } \\
& \text { AGC_Vmin }=-1.56 \\
& \text { AGC_Vmax }=1700.00 \\
& \text {; min. Voltage [V] } \\
& \mathrm{HV}=\text { fltarr(7,nMJFs }) \\
& \text { Loop }=\text { fltarr }(7, \text { nMJFs }) \\
& \text { Gain }=\text { fltarr }(7, \mathrm{nMJFs})
\end{aligned}
$$




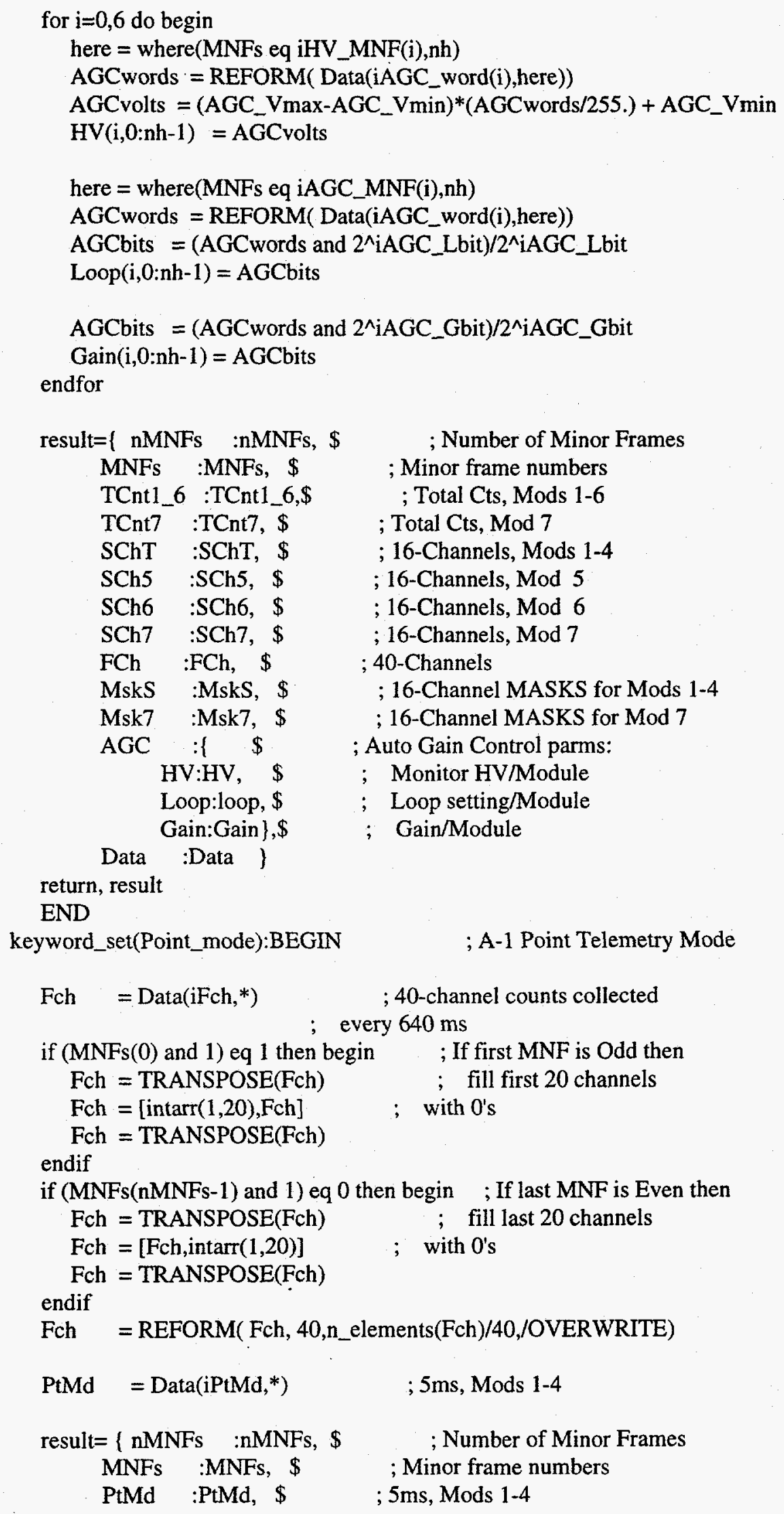




\[ \begin{array}{l}\text { FCh } \\ \text { Data }\end{array} \text { :DCh, } \$ \text {; } \]
return, result
END
SE: message,/INF,'No Telemetry Mode specified.'
CASE

end

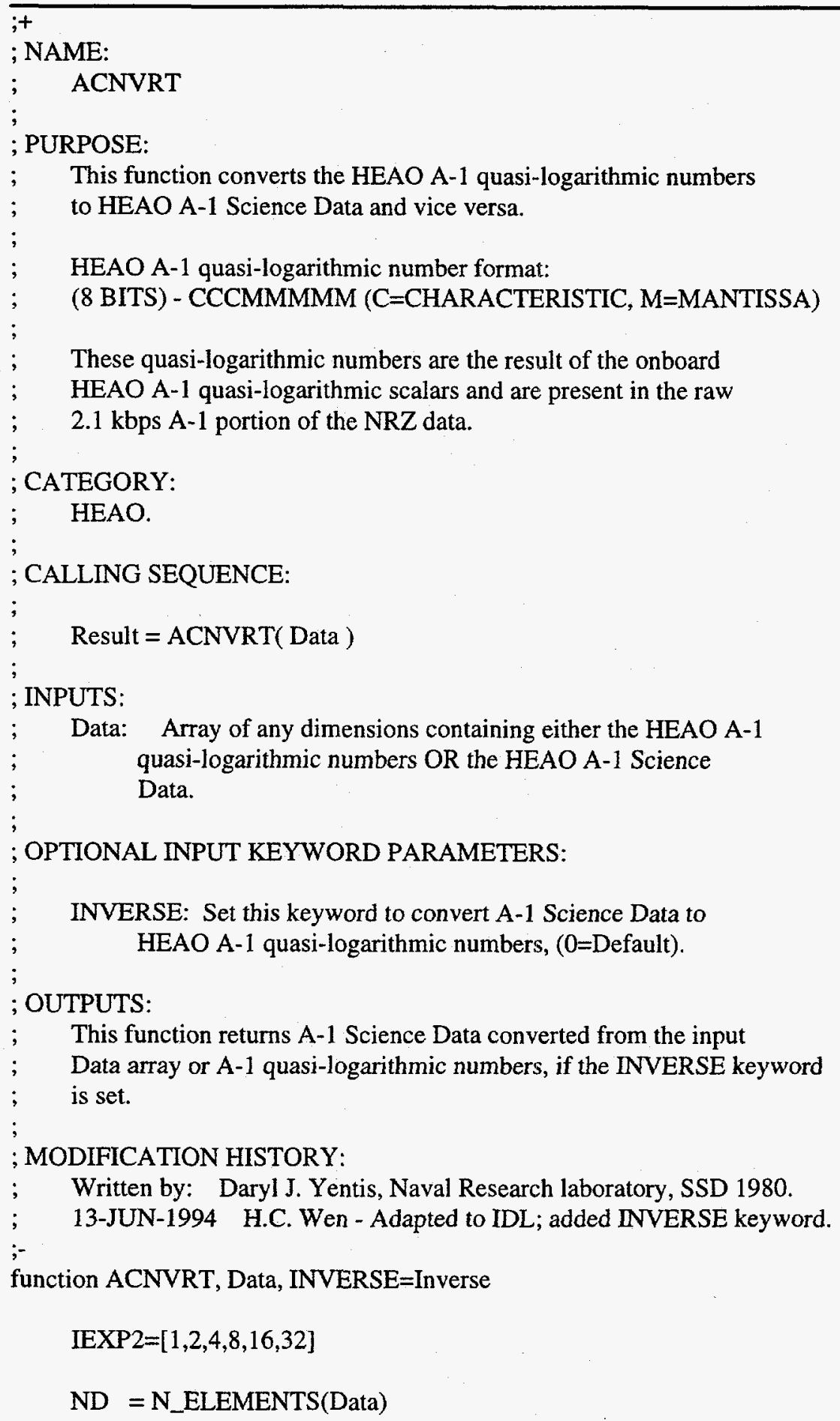

This function returns A-1 Science Data converted from the input Data array or A-1 quasi-logarithmic numbers, if the INVERSE keyword is set. 
if keyword_set(INVERSE) then begin

ID $=$ FIX (Data)

h64 = where(Data gt 64, n64)

if ( $\mathrm{n} 64 \mathrm{gt} 0$ ) then begin

D64 = FIX (Data(h64))

char $=$ REPLICATE $(2$, n64 $)$

h127 $=$ where (D64 gt 127, n127)

while (n127 gt 0 ) do begin

D64(h127) $=$ ISHFT (D64(h127),-1)

$\operatorname{char}(\mathrm{h} 127)=\operatorname{char}(\mathrm{h} 127)+1$

h127 = where(D64 gt 127, n127)

endwhile

mant $=$ ISHFT $($ D64-64,-1)

ID $($ h64 $)=$ ISHFT $($ char ,5) + mant

endif

return, ID

endif

ID $=$ FIX(Data) $\quad$; convert to 16 -bit words

; FINISHED IF $D=0$ OR $C=0$ OR 1

here $=$ WHERE $($ ID ge ' 100 ' $\mathrm{O}$, nconvert $)$

if (nconvert gt 0 ) then begin

; CHARACTERISTIC (3-BITS)

; IC=ISHIFT(ID,-5).AND.7K

ICs $=\mathrm{ID}($ here $) /{ }^{\prime} 40^{\prime} \mathrm{O}$

; MANTISSA (5-BITS)

$\mathrm{IMs}=\mathrm{ID}$ (here) and ${ }^{\prime} 37^{\prime} \mathrm{O}$

; $\mathrm{D}=(2 *(\mathrm{M}+32)+1)^{*}(2 * *(\mathrm{C}-2))$

; DATA(I) $=$ ISHIFT $(2 *$ IM +65 ,IC- 2$)$

$\mathrm{ID}($ here $)=(2 * \mathrm{IMs}+65) * \mathrm{IEXP} 2$ (ICs-2)

endif

return, ID

end 
Appendix B

\section{HEAO A-1 High Bit Rate Data Archive}

\section{B.1 Summary of the HBR Data}

A summary of all the useable HEAO A-1 HBR data is shown in Table B.2. Each row represents a different scanned or pointed observation. A total of 23 separate observations from November 1977 to January 1979 are listed. Most of these observations were pointed and a total of 13 different X-ray targets were observed. Details of the instrument configuration are provided in columns 11-12, specifying which Modules collected the data in which telemetry and data format modes. The list of observations in Table B.2 is restricted to those collected in the Bit mode, since the data formats of the other two modes, Word and Frame are not understood. The raw HBR data file locations at NRL and at SLAC are specified by the information listed in the first four columns. All of the data processing steps described in Chapter 3 have been applied to each observation listed.

Any observations that are subsequently referenced will follow the following naming convention: the NRL sequence number, tape copy and file number, as described in columns 3-5 of Table B.2. For example, there are two pointed observations of Cygnus $\mathrm{X}-1$. The first observation, analyzed by Meekins' et. al., is listed on row 12 of Table B.2 and will be referenced as 086_7_7. The second observation listed on row 8 will be referenced as 015_2_3. 


\begin{tabular}{|c|c|c|c|c|}
\hline Column & Heading & \multicolumn{3}{|c|}{ Description } \\
\hline 1 & $\begin{array}{l}\text { SLAC Vol } \\
\text { Ser }\end{array}$ & \multicolumn{3}{|c|}{ Volume serial number of the SLAC silo cartridge. } \\
\hline 2 & SLAC Seq & \multicolumn{3}{|c|}{ The sequence number on the SLAC silo cartridge } \\
\hline 3 & NRL Seq & \multicolumn{3}{|c|}{$\begin{array}{l}\text { The sequence number on the NRL magnetic tapes } \\
\text { containing the original analog HBR data. }\end{array}$} \\
\hline 4 & COPY Tape & \multicolumn{3}{|c|}{$\begin{array}{l}\text { One of the NRL magnetic tapes containing a copy of the } \\
\text { digitized HBR data. }\end{array}$} \\
\hline 5 & File & \multicolumn{3}{|c|}{ The file number on that NRL magnetic tape. } \\
\hline 6 & $\operatorname{Rev}$ & \multicolumn{3}{|c|}{ HEAO satellite revolution number. } \\
\hline 7 & Pass & \multicolumn{3}{|c|}{ Ground station pass, abbreviation. } \\
\hline 8 & Time & & & \\
\hline 9 & Day & \multicolumn{3}{|c|}{ Day of the year. } \\
\hline 10 & Date & & & \\
\hline 11 & Configuration & $\begin{array}{l}\text { HBRh } \\
\text { PMmh } \\
\text { MODn } \\
\text { FCON } m f\end{array}$ & $\begin{array}{l}\text { HBR mode } \\
\text { Modules selected } \\
\text { for HBR data } \\
\text { NRZ mode } \\
\text { Modules selected } \\
\text { for 40-channel } \\
\text { analyzer }\end{array}$ & $\begin{aligned} h & =(\mathrm{B}) \mathrm{it},(\mathrm{F}) \mathrm{rame} \text { or }(\mathrm{W}) \text { ord } \\
m h & =(\mathrm{SCA})-\text { mods } 1-4 \\
& =(\mathrm{SEV})-\text { module } 7 \\
n & =(\mathrm{P} 0)-5 \mathrm{~ms} \\
& =(\mathrm{SC})-320 \mathrm{~ms} \\
m f & =\text { List of modules, } 1-4\end{aligned}$ \\
\hline 12 & Sat Mode & \multicolumn{3}{|c|}{ HEAO satellite mode,$(P)=$ point,$(S)=$ scanning } \\
\hline 13 & Min & \multicolumn{3}{|c|}{ Length of the processed HBR data in minutes. } \\
\hline 14 & Target & \multicolumn{3}{|c|}{$\begin{array}{l}\text { Description of the celestial object; the character(s) in } \\
\text { parentheses (e.g. (U)) follows the van Paradijs' Catalogue of } \\
\text { X-ray Binaries letter code, e.g. U - ultra-soft X-ray } \\
\text { spectrum). }\end{array}$} \\
\hline
\end{tabular}

Table B.1 Description of the column headings in Table B.2. 


\begin{tabular}{|c|c|c|c|c|c|c|c|c|c|c|c|c|c|c|}
\hline & 1 & 2 & 3 & 4 & 5 & 6 & 7 & 8 & 9 & 10 & 11 & 12 & 13 & 14 \\
\hline & $\begin{array}{l}\text { SLAC } \\
\text { Vol Ser }\end{array}$ & \begin{tabular}{|l} 
SLAC \\
Seq \\
\end{tabular} & $\begin{array}{l}\text { NRL } \\
\text { Seq } \\
\end{array}$ & $\begin{array}{l}\text { COPY } \\
\text { Tape } \\
\end{array}$ & File & Rev & Pass & Time & Day & Date & Configuration & $\begin{array}{c}\text { Sat } \\
\text { Mode }\end{array}$ & Min & Target \\
\hline 1 & RY2071 & 23 & 20 & 2 & 8 & 6250 & $\mathbf{H}$ & 4:32:00 & 262 & 19-Sep-78 & HBRB, PMSCA, MODP0 & $\mathbf{P}$ & 8.3 & 4U1728-34, 1728-337, LMXB, NS, (BA) \\
\hline 2 & RY2071 & 10 & 10 & 1 & 9 & 5507 & G & 12:58:00 & 214 & 2-Aug-78 & HBRB, PMSEV, MODP0 & $\mathbf{P}$ & 9.0 & Cas A, 2321+585, SNR \\
\hline 3 & RY2071 & 9 & 36 & 4 & 2 & 5470 & $\mathbf{H}$ & 3:33:39 & 212 & 31-Jul-78 & HBRB, PMSEV, MODPO & $\mathbf{P}$ & 5.6 & Cen X-3, 1119-603, HMXB, NS, (P) \\
\hline 4 & RY2071 & 16 & 12 & 1 & 11 & 5776 & $Q$ & 19:22:00 & 231 & 19-Aug-78 & HBRB, PMSEV, MODPO & $\mathbf{P}$ & 2.7 & Cir X-1, 1516-569, LMXB, NS, (TB) \\
\hline 5 & RY2070 & 16 & 25 & 3 & 3 & 5775 & $\mathbf{I}$ & $17: 40: 12$ & 231 & 19-Aug-78 & HBRB, PMSEV, MODPO & $\mathbf{P}$ & 7.3 & Cir X-1, 1516-569, LMXB, NS, (TB) \\
\hline 6 & RY2072 & 5 & 79 & 7 & 3 & 8000 & G & 17:48:00 & 8 & 8-Jan-79 & HBRB, PMSCA, (MODP0) & $\mathbf{P}$ & 8.5 & Cir X-1, 1516-569, LMXB, NS, (TB) \\
\hline 7 & RY2069 & 12 & 2 & 1 & 2 & 531 & $\mathbf{H}$ & 14:49:00 & 258 & 15-Sep-77 & HBRB,PMSCA,FCON1 234, ,MODSC & $\mathbf{s}$ & 6.7 & Crab, $0531+219, \mathrm{NS},(\mathrm{P})$ \\
\hline 8 & RY2071 & 29 & 15 & 2 & 3 & 7056 & I & 17:26:00 & 313 & 9-Nov-78 & HBRB, PMSCA, MODP0 & $\mathbf{P}$ & 8.5 & Cyg X-1, 1956+350, HMXB, BHC, (U) \\
\hline 9 & RY2069 & 28 & 38 & 14 & 4 & 1331 & $\mathbf{H}$ & 9:54:50 & 310 & 6-Nov-77 & HBRB, PMSCA,FCON3 4,(MODSC) & $\mathbf{S}$ & 5.7 & Cyg X-1, 1956+350, HMXB, BHC, (U) \\
\hline 10 & RY2069 & 29 & 38 & 14 & 5 & 1331 & $\mathbf{H}$ & 9:54:50 & 310 & 6-Nov-77 & HBRB, PMSCA,FCON3 4,(MODSC) & $s$ & 5.7 & Cyg X-1, 1956+350, HMXB, BHC, (U) \\
\hline 11 & RY2070 & 3 & 42 & 12 & 6 & 1349 & G & 12:14:50 & 311 & 7-Nov-77 & HBRB, PMSCA, FCON3 4, (MODSC) & $S$ & 5.5 & Cyg X-1, 1956+350, HMXB, BHC, (U) \\
\hline 12 & RY2070 & 26 & 86 & 7 & 7 & 4147 & 1 & $2: 47: 40$ & 127 & 7-May-78 & HBRB, PMSCA, MODSC & $\mathbf{P}$ & 8.5 & Cyg X-1, 1956+350, HMXB, BHC, (U) \\
\hline 13 & RY2071 & 1 & 8 & 1 & 7 & 4542 & H & 12:29:00 & 152 & 1-Jun-78 & HBRB, PMSEV, MODPO & $\mathbf{P}$ & 7.4 & Cyg X-3, 2030+407, HMXB, BHC \\
\hline 14 & RY2071 & 17 & 24 & 3 & 2 & 5834 & 1 & 12:18:10 & 235 & 23-Aug-78 & HBRB, PMSCA, MODSC & $\mathbf{P}$ & 8.1 & Gamma Cas, 0053+604, HMXB \\
\hline 15 & RY2071 & 13 & 1 & 1 & 1 & 5713 & $\mathbf{H}$ & 18:26:45 & 227 & 15-Aug-78 & HBRB, PMSEV, MODP0 & $\mathbf{P}$ & 8.2 & Her X-1, $1656+354$, LMXB, NS, (P) \\
\hline 16 & RY2071 & 12 & 11 & 1 & 10 & 5688 & $\mathrm{G}$ & $3: 43: 50$ & 226 & 14-Aug-78 & HBRB, PMSEV, MODP0 & $\mathbf{P}$ & 7.7 & Her X-1, 1656+354, LMXB, NS, (P) \\
\hline 17 & RY2070 & 21 & 26 & 3 & 4 & 3102 & $\mathrm{H}$ & 18:00:50 & 59 & 28-Feb-78 & HBRB, PMSEV, MODP0 & $\mathbf{P}$ & 8.2 & Her X-1, 1656+354, LMXB, NS, (P) \\
\hline 18 & RY2071 & 15 & 91 & 7 & 9 & 5759 & 1 & 17:00:47 & 230 & 18-Aug-78 & HBRB, PMSEV, MODP0 & $\mathbf{P}$ & 8.1 & Her X-1, 1656+354, LMXB, NS, (P) \\
\hline 19 & RY2071 & 24 & 18 & 2 & 6 & 6338 & I & 19:49:00 & 267 & 24-Sep-78 & HBRB, PMSEV, (MODP0) & $\mathbf{P}$ & 8.7 & $\mathrm{MX} 1730-63$ \\
\hline 20 & RY2071 & 18 & 23 & 3 & 1 & 5308 & $\mathrm{G}$ & 18:17:00 & 201 & 20-Jul-78 & HBRB, PMSEV, MODP0 & $\mathbf{P}$ & 7.9 & New Nova, 0115+634, HMXB, BHC, (TP) \\
\hline 21 & RY2071 & 5 & 77 & 7 & 1 & 5106 & $\mathrm{H}$ & $18: 43: 00$ & 188 & 7-Jul-78 & HBRB, PMSCA, MODPO & $\mathbf{s}$ & 10.6 & Nova, Mag. Clouds \\
\hline 22 & RY2071 & 20 & 13 & 2 & 1 & 6018 & $\mathrm{H}$ & $7: 19: 00$ & 247 & 4-Sep-78 & HBRB, PMSEV, MODPO & $\mathbf{P}$ & 7.8 & OAO 1653-40 \\
\hline 23 & RY2071 & 25 & 19 & 2 & 7 & 6367 & I & 16:17:00 & 269 & 26-Sep-78 & HBRB, PMSCA, MODPO & $\mathbf{P}$ & 7.3 & OAO 165340 \\
\hline
\end{tabular}

Table B.2 Summary of all usable HBR data files. Columns 1-2 identifies the file location in the SLAC silos. Columns 2-4 identifies the file location among the digitized NRL tapes. Columns 6-10 provides date and time information. Columns 11-14 describes the telemetry mode, length of data and target name. For a more detailed description of each column heading, see Table B.1. 


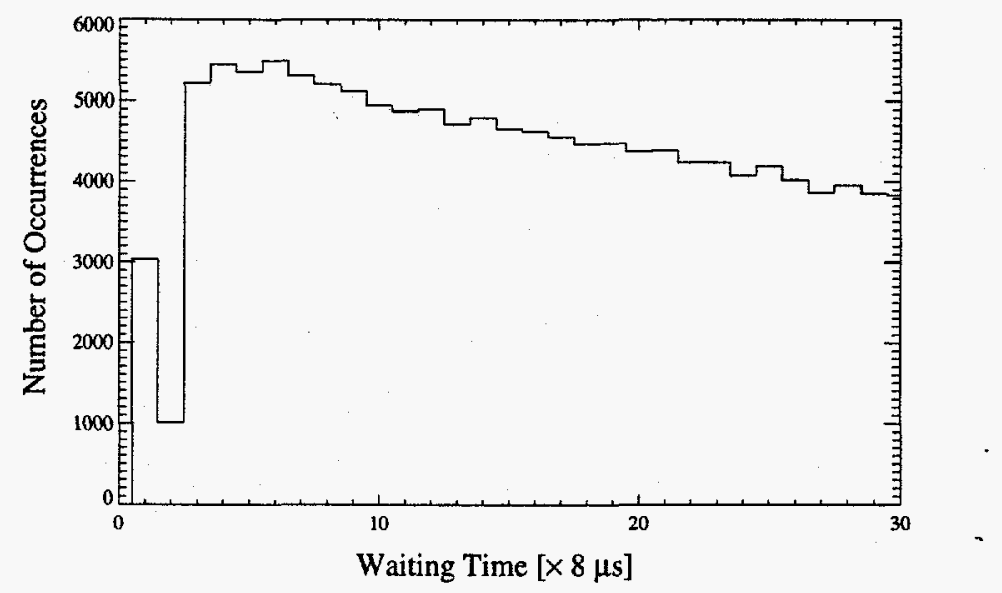

Fig. B.1 Waiting time distribution for an uncorrected HBR data file showing an anomalous number of pairs of events with a waiting time of 1 .

\section{B.2 Data Screening}

The process of data screening involves identifying artifacts in the data and correcting for them. One such artifact as described below was found in the HBR data and subsequently corrected for. The HBR data is checked for additional artifacts by comparing the HBR and NRZ data over periods when they simultaneously observed a target.

\section{B.2.1 Correcting for Adjacent Pairs}

An examination of the waiting time distributions of the HBR data showed an anomalous excess of occurrences at the 8 microsecond waiting time, as Figure B.1 demonstrates. These waiting times correspond to events that are recorded in two sequential 8 microsecond time bins and will be called adjacent pairs. Before these adjacent pairs can be identified as anomalous, binning effects must be first considered. If the data are binned at an 8 microsecond resolution, the location of an event in such a bin is unknown. In units of 8 microsecond bins, a binned waiting time of $n$ corresponds to an uncertainty in the actual waiting time of $[n-1, n+1]$. Given a dead time of $19 \mu \mathrm{s}$, as described in Section 3.4.1, the smallest binned waiting time possible is 2 . However, the 
adjacent pairs have a binned waiting time of 1 . For each observation, these adjacent pairs represent about $0.6 \%$ of the total number of observed events.

The adjacent pairs have been found to occur at same position in the data format of the raw digitized data. As described in Appendix A.1, the data format of the raw 8 microsecond data consists of 4096 16-bit words for each $320 \mathrm{~ms}$ record. Ten of the bits from each word represent 10 sequential 8 microsecond time bins, with a bit set if a photon is detected in that time bin. The adjacent pairs occur only across adjacent words, the last time bit of one word and the first time bit of the next word.

To determine which events in an adjacent pair should be removed, waiting time distributions were made for two possibilities. When one event is removed, the resulting distribution of waiting times between the remaining event and its nearest neighbor is exponential. When both events are removed, the distribution of waiting times between the adjacent pair's nearest neighbors follows a gamma distribution

$$
f(t ; \lambda, k)=\frac{t^{k-1} \lambda^{k} e^{-\lambda t}}{(k-1) !}
$$

at $k=2$. Here, $t$ is the waiting time and $\lambda$ is $k \rho$. Equation [B.1] is the distribution for a Poisson process of waiting times from a particular event to the following $k^{\text {th }}$ event. Namely, distributions for both possibilities are consistent with identifying only one event from the adjacent pair as artificial.

The HBR data were corrected by removing one event from each adjacent pair present. For a given adjacent pair, the event removed was chosen pseudo-randomly by selecting the event with the closest neighboring event outside the adjacent pair. The KILLAPE routine performed this data correction and was called within the CTIFMT routine. 


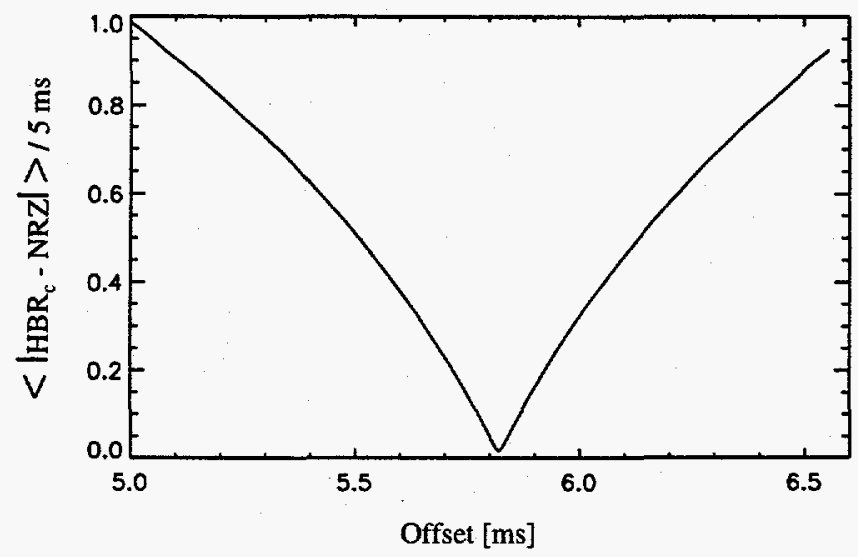

Fig B.2 The average of the absolute valued differences between the corrected HBR and NRZ time series as a function of various offsets. The minimum occurs at an offset of $5.82 \mathrm{~ms}$. (Data file: 015_2_3)

\section{B.2.2 Data Integrity Check}

After applying corrections to the adjacent pairs, the HBR data were then compared to the NRZ data of the same period. The first part in this check was finding the correct offset between the start of the HBR and NRZ data. The NRZ data lagged the HBR data by approximately the length of the NRZ telemetry resolution ( 5 or $320 \mathrm{~ms}$ ). This length of time is required to collect one time bin of NRZ data and then additional time is needed to process it. An offset was added to the event times of the HBR data and the resulting offset times were histogrammed at the same time resolution as the NRZ data. The two time series were compared by averaging the absolute value of their difference. The offset was varied until a minimum was found. For the Cygnus X-1 data set, 015_2_3 the NRZ time resolution was $5 \mathrm{~ms}$, and as Figure B.2 shows, this minimum occurs at a $5.82 \mathrm{~ms}$ offset.

The difference between the NRZ and corrected HBR time series with the latter adjusted for a $5.82 \mathrm{~ms}$ offset is shown in Figure B.3 for a sample of time bins. Discrepancies of \pm 1 count/bin are observed for $\sim 0.3 \%$ of the entire data set. The 


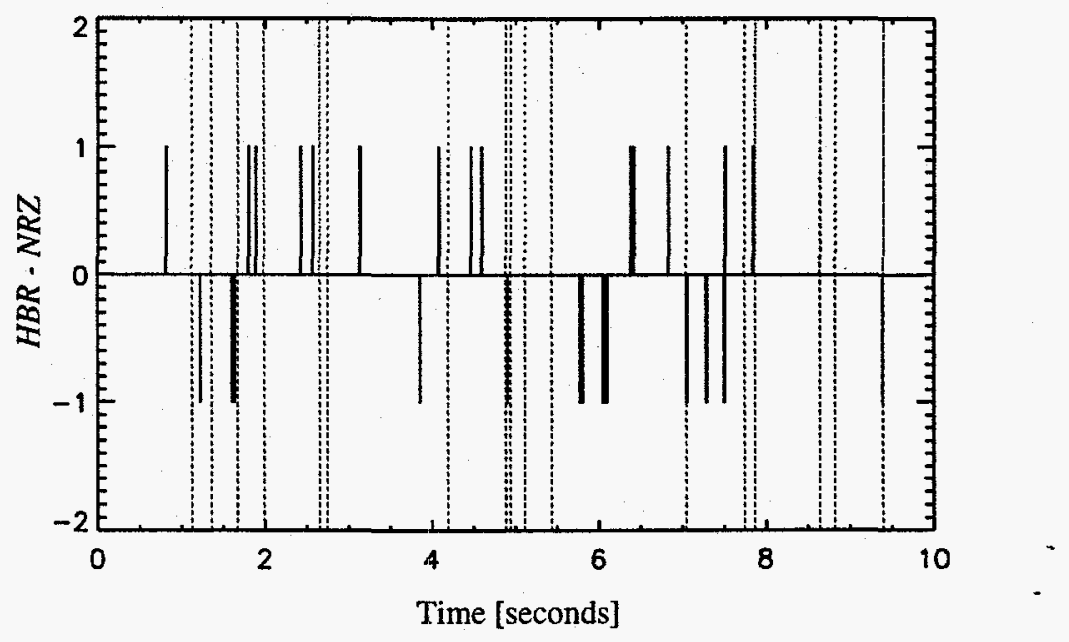

Fig. B.3 The difference between the $5 \mathrm{~ms}$ resolution time series of the corrected HBR and NRZ data for the data set, 015_2_3. The adjacent pairs have been corrected and a $5.82 \mathrm{~ms}$ offset has been added. The dashed vertical lines mark the locations in time of the adjacent pairs. There are 27 bins with \pm 1 count discrepancies and 18 bins with an Adjacent Pair out of a total of 10,162 counts.

locations of adjacent pairs are show in Figure B.3 to be uncorrelated with the remaining discrepancies.

A measure of the significance of the remaining discrepancies is the average and rms average of the residuals,

$$
R=\frac{H B R-N R Z}{\sigma_{H B R-N R Z}}=\frac{H B R-N R Z}{\sqrt{H B R+N R Z}}
$$

where $H B R$ and $N R Z$ are the number of counts/bin in HBR and NRZ time series, respectively. For the 015_2_3 data set, $\langle R\rangle=-2 \times 10^{-4}$ and $\langle R\rangle_{r m s}=0.04$, indicating a uniform distribution of \pm 1 count/bin discrepancies at a level much smaller than the uncertainties due to Poisson statistics. 
Appendix C

\section{Dead-Time-Distorted Waiting Time Distributions}

\section{C.1 Probability Distribution of Binned Waiting Times}

Let $\mathrm{T}$ be the time resolution or width of the time bins in a given apparatus. The dead time of the apparatus is non-extended and greater than $\mathrm{T}$ for this derivation. All events originate from a Poisson process with a true count rate $\rho$. Assume an event is detected in the $0^{\text {th }}$ time bin. The probability of the next event occuring $\mathrm{n}$ time bins away is

$$
P(n)=\int f_{1}(s) P_{2}(s) d s
$$

where $s$ is the period from the start of the $0^{\text {th }}$ bin, $f_{1}(s)$ is the density of the first event occurring at $s$ and $P_{2}(s)$ is the probability of detecting the second event in the $\mathrm{n}^{\text {th }}$ bin if the first event occurs at $s$. The location of the first event in the $0^{\text {th }}$ time bin is random, so the density function is simply

$$
f_{1}(s)=\frac{1}{T}
$$

and the probability for the second event is given by the integral of waiting time density in the presence of a non-extended dead time $\tau$, 


$$
P_{2}(s)=\int U\left(s^{\prime}-\tau\right) \rho e^{-\rho\left(s^{\prime}-\tau\right)} d s^{\prime}
$$

where $s^{\prime}$ is the waiting time between the two events and $U$ is the unit-step function.

The limits in equations [C.1] and [C.3] depend on how close the $\mathrm{n}^{\text {th }}$ bin is to the dead time $\tau$. Defining $\kappa$ as the largest integer $\leq \tau / T$, there are four cases to consider.

case 1)

case 2) $n=\kappa$

case 3) $\quad n=\kappa+1$

case 4) $n>\kappa+1$

$$
s^{\prime} \in[0,0],
$$

$s^{\prime} \in[\tau,(n+1) \mathrm{T}-s]$,

$s^{\prime} \in[n \mathrm{~T}-\mathrm{s},(n+1) \mathrm{T}-s]$,

$s^{\prime} \in[\tau,(n+1) \mathrm{T}-s]$,

$s^{\prime} \in[\mathrm{nT}-\mathrm{s},(n+1) \mathrm{T}-s]$

$$
s \in[0,0]
$$$$
s \in[0,(n+1) \mathrm{T}-\tau]
$$$$
s \in[0, n \mathrm{~T}-\tau]
$$$$
s \in[n \mathrm{~T}-\tau, \mathrm{T}]
$$

$$
s \in[0, \mathrm{~T}]
$$

Inserting these limits into equations [C.1] and [C.3] yields the following solution

$$
P(n)= \begin{cases}0, & 0 \leq n \leq \kappa-1, \\ 1-\varepsilon-\left(1-e^{-\mu(1-\varepsilon)}\right) / \mu, & n=\kappa, \\ \varepsilon+\left(1+e^{-\mu(1-\varepsilon)}\left(e^{-\mu}-2\right)\right) / \mu, & n=\kappa+1, \\ \left(\frac{\sinh (\mu / 2)}{\mu / 2}\right)^{2} \mu e^{-\rho(n T-\tau)}, & n>\kappa+1\end{cases}
$$

where $\mu=\rho \mathrm{T}$ and $\varepsilon=\tau / \mathrm{T}-\kappa$. Equation [C.4] is the probability distribution of binned waiting times for a Poisson process in the presence of a non-extended dead time $\tau$ and a time resolution $\mathrm{T}$.

\section{C.2 Waiting Time Density of the USA Perimeter Veto Problem}

The preflight USA perimeter veto logic failed to properly reset itself for noncoincidence charged particle events. The first photon event following any perimeter veto event that had not self-cleared is vetoed. This resulted in an effective variable dead time in addition to the normal detector dead time. 
Before proceeding, several definitions are in order to facilitate the derivation of the waiting time density for a Poisson source in the presence of this electronics malfunction. Let $\rho_{\gamma}$ and $\rho_{v}$ represent the count rates of the Poisson source and the perimeter veto events that were not self-cleared, respectively. The normal detector dead time will be neglected in this derivation to simplify the calculation. Let $\mathrm{P}_{0}(x, T)$ represent the probability that no events of type $x$ ( $\gamma$ or v) occur in an interval of time $T$ and $f_{x}(T)$ represents the probability density of events of type $x$ arriving a waiting time $T$ later.

The waiting time density for this problem is the sum of the waiting time densities for all possible ways that veto and photon events could have arrived between the waiting time between two detected photon events. The first possibility is that no veto events had arrived. The waiting time density for this configuration is

$$
f_{00}=\mathrm{P}_{0}(v, T) f_{\mathrm{\gamma}}(T)
$$

where $T$ is the waiting time between the two detected photon events. The second possibility is that one veto event had arrived during the interval $T$. Since a second photon was detected at the end of the interval $T$, that means one other photon event must have arrived between the veto event and the second photon. The waiting time density for this configuration is

$$
f_{11}=\int P_{0}\left(\gamma, x_{1}\right) f_{v}\left(x_{1}\right) P_{0}\left(v, T-x_{2}\right) f_{\gamma}\left(x_{2}\right) f_{\gamma}\left(T-\left(x_{1}+x_{2}\right)\right)
$$

Repeating this logic for additional veto events arriving between two observed photons events, the general waiting time density for $v$ veto events and $x$ photon events arriving between two observed photon events can be derived to be

$$
f_{v x}(T)=\left(\begin{array}{c}
v-1 \\
x-1
\end{array}\right) \frac{\left(\rho_{v} T\right)^{v}\left(\rho_{\gamma} T\right)^{x}}{(v+x) !} \rho_{\gamma} e^{-\left(\rho_{\nu}+\rho_{\gamma}\right) T}
$$


The actual waiting time density for this perimeter veto model (PVM) is the sum off all the waiting time densities defined by [C.7] over all possible combinations of veto and photon events that can occur between two observed photon events.

$$
f_{p v m}(T)=\sum_{v=1}^{\infty} \sum_{x=1}^{v} f_{v x}(T)
$$


Appendix D

\section{Testing of the USA Collimators}

\section{D.1 Introduction}

As part of the development of the USA instrument, SLAC has assumed responsibility for the design, construction and testing of the USA collimator. The collimator is a honeycomb of narrow tubes that restricts the solid angle of detected X-rays to directions within the collimator's field of view. To facilitate construction and testing, a modular design was adopted, dividing the collimator into eight identical modules for each detector. Each module was manufactured from layers of rolled, half-hexagonal copper sheets that were epoxied and cured together to form honeycomb tubes. Figure D.1 shows an image of one of the constructed USA collimators.

The testing of the USA collimators consisted of measuring the acceptance of each module to X-rays incident at given relative angles. Rotations about a vertical $\mathrm{Y}$-axis (yaw or $\phi$ ) and a horizontal $\mathrm{X}$-axis (pitch or $\theta$ ) described these relative angles. All angles are relative to the axis normal to the module's face (i.e. the Z-axis). The module's acceptance as a function of pitch and yaw will be called the response function. For each module, an elliptical cone was fitted to the measured response function to determine its full width half-maximum (FWHM) and the location of its peak in pitch and yaw. These parameters 


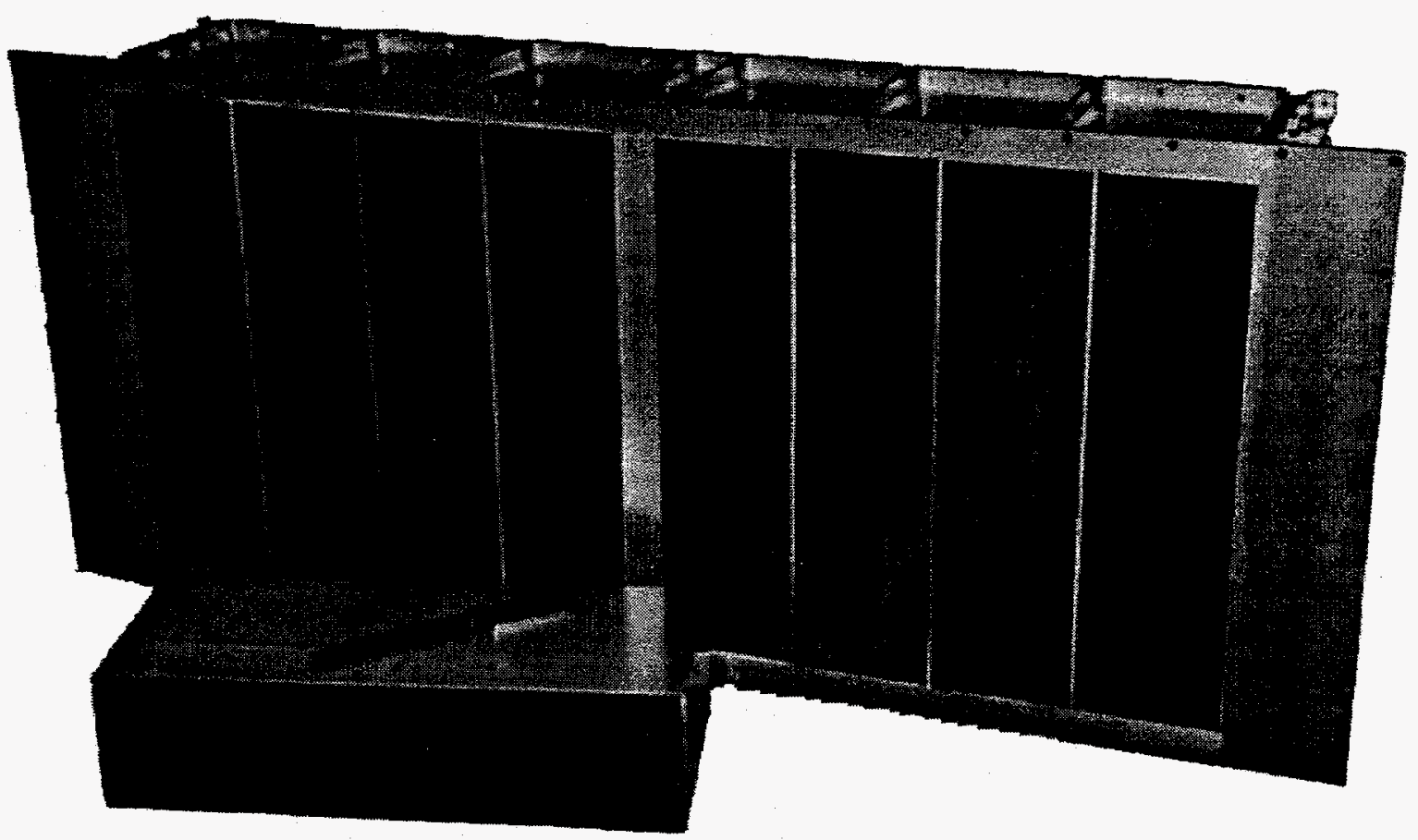

Fig. D.1 One of three USA collimators constructed at SLAC. Eight modules can be seen in the collimator frame stacked in two groups of four. An additional module separate from the collimator is also shown. Each module is framed by thin aluminum (copper anodized) bridge sheets to help support the honeycomb structure.

were used to help select/match the modules that would form the three final collimators ( 2 flight, 1 spare) by simulating the response function expected for a given configuration of eight modules.

A small experiment nicknamed X-Ray Cannon (XRC) was performed at SLAC to individually measure each collimator module response function. Each module was set into a miniaturized USA detector with a goniometer supporting the entire assembly. Counts from an X-ray beam were then measured at different pitch and yaw. The following Appendix will describe the setup, calibration, data acquisition and analysis of the XRC experiment. 


\section{D.2 XRC Setup}

The setup of the X-Ray Cannon experiment consisted of two major components: a source and a detector assembly. The source assembly consisted of building an apparatus that would deliver an X-ray beam. To detect those X-rays a simple detector was assembled along with the supporting apparatus, electronics and data acquisition.

\section{D.2.1 Source Assembly}

The X-ray beam delivered to the detector satisfied several criteria. A thin $4 " \times 4 "$ sheet of $55 \mathrm{Fe}$ with an activity of $25 \mathrm{mC}$ was chosen for the X-ray source. Its energy (6 $\mathrm{keV} K_{\alpha}$ ) lies within USA's energy sensitivity, $2-15 \mathrm{keV}$. Its surface area covered a significant portion of the $3^{\prime \prime} \times 11$ " collimator module face. A long pipe then collimated this source into a beam of $\mathrm{X}$-rays. The length was chosen to give a beam divergence $\left(0.07^{\circ} \mathrm{FWHM}\right)$ that was smaller than the resolution which was chosen to measured the response function, $0.1^{\circ}$.

Other methods for delivering an X-ray beam with a higher intensity were also considered. However, the development of extensive radiation safety infrastructure required for sources with significant activity $(\sim 0.1-1 \mathrm{C})$ dictated a practical limit to the source strength. The source chosen was the hottest source available that required minimal radiation safety precautions.

With the given source area and beam divergence, a 200' long pipe or "cannon" was assembled with an effective 1.5" inner radius. A straight section of the PEP Machine tunnel, adjacent to $\mathbb{R} 12$ was chosen to contain this pipe. The cannon was constructed from 20' segments of 2" inner radius PVC pipe and supported every $10^{\prime}$ by two 5/8" diameter steel rods clamped to cable tray support beams above and threaded into a pipe hanger below. As Figure D. 2 shows, one rod was vertical and the other at $45^{\circ}$ to provide rigid support in both vertical and horizontal directions. To eliminate any small angle scattering of X-rays off the PVC surface, aluminum baffle discs with a $1.5^{\prime \prime}$ inner radius were positioned at the end of each PVC pipe segment. 


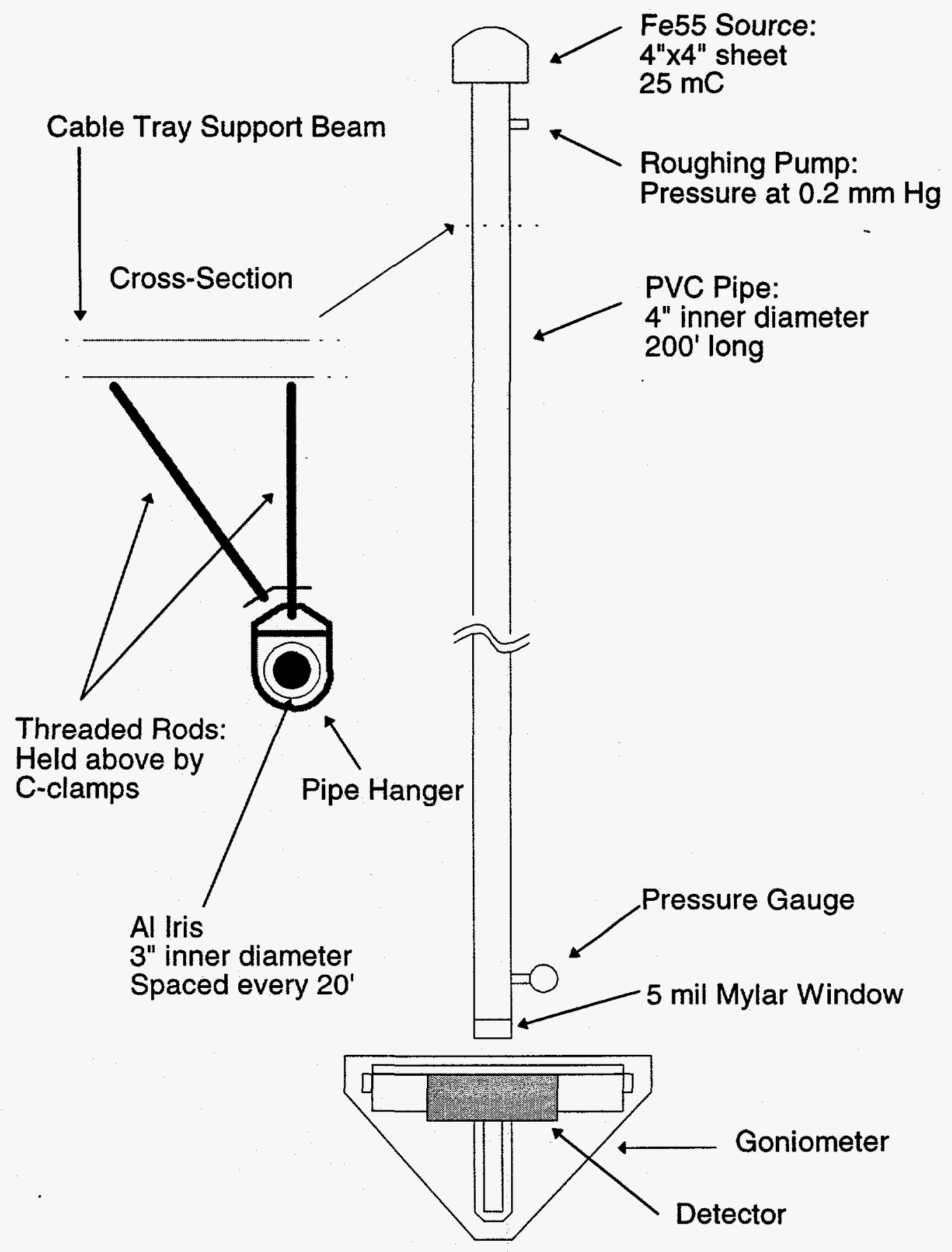

Fig. D.2 Layout of the XRC experiment. 
A roughing pump evacuated the pipe volume to $200 \mu \mathrm{Hg}$ of pressure to help minimize the attenuation of the X-rays over the length of the pipe. To contain this vacuum, a 5 mil mylar window sealed the detector end while an end cap with the source positioned inside sealed the other. The mylar window was supported between two aluminum discs epoxied together along with a thin bar across the diameter.

\section{D.2.2 Detector Assembly}

The detector assembly was designed about the geometry defined by the $\mathrm{X}$-ray beam and the collimator modules. For the $\mathrm{X}$-ray detector, a small single-wire proportional wire chamber (PWC) was constructed to allow a collimator module to be mounted on it. A goniometer was designed and machined to enable relative orientations between the collimator and beam axes. In addition, a 4-sided scintillator was built to help veto cosmic ray events.

To mount a collimator module onto the PWC, the $3 " \times 11^{\prime \prime} \times 4 \frac{1}{2}$ " module was first set into an aluminum frame and then bolted against the PWC with a $\frac{1}{2}$ mil mylar window in between. An evaporated aluminum film covered the PWC side of the mylar window, insuring a uniform ground over the entire inner surface of the PWC. A single $30 \mu \mathrm{m}$ gold-plated wire stretched across the longest dimension of the proportional wire chamber's $3 "$ x $11 " \times 2 \frac{1}{2}$ " active volume. Two gas couplings allowed P-10 gas (10\% $\mathrm{CH}_{4}, 90 \% \mathrm{Ar}$ ) to flow continuously through the chamber's active volume at $\sim 1 \mathrm{~cm}^{3} \mathrm{~s}^{-1}$.

Supporting the PWC and collimator unit was a goniometer that was designed with two perpendicular axes of rotation. As shown in Figure C.2, a double layered "T"-shaped geometry provided the two independent degrees of freedom. Two small aluminum blocks fastened to the bottom " $\mathrm{T}$ " layer provided gimbals support to the top " $\mathrm{T}$ " layer, enabling rotations in the vertical plane. A pivot at the " $\mathrm{T}$ " intersection of the bottom layer allowed rotations in the horizontal plane. Rotations of the goniometer in the horizontal and vertical planes will be referred to by the variables $\phi$ and $\theta$, respectively. In relation to the USA instrument, a rotation in the $\phi$ and $\theta$ directions correspond to yaw and pitch, respectively. 


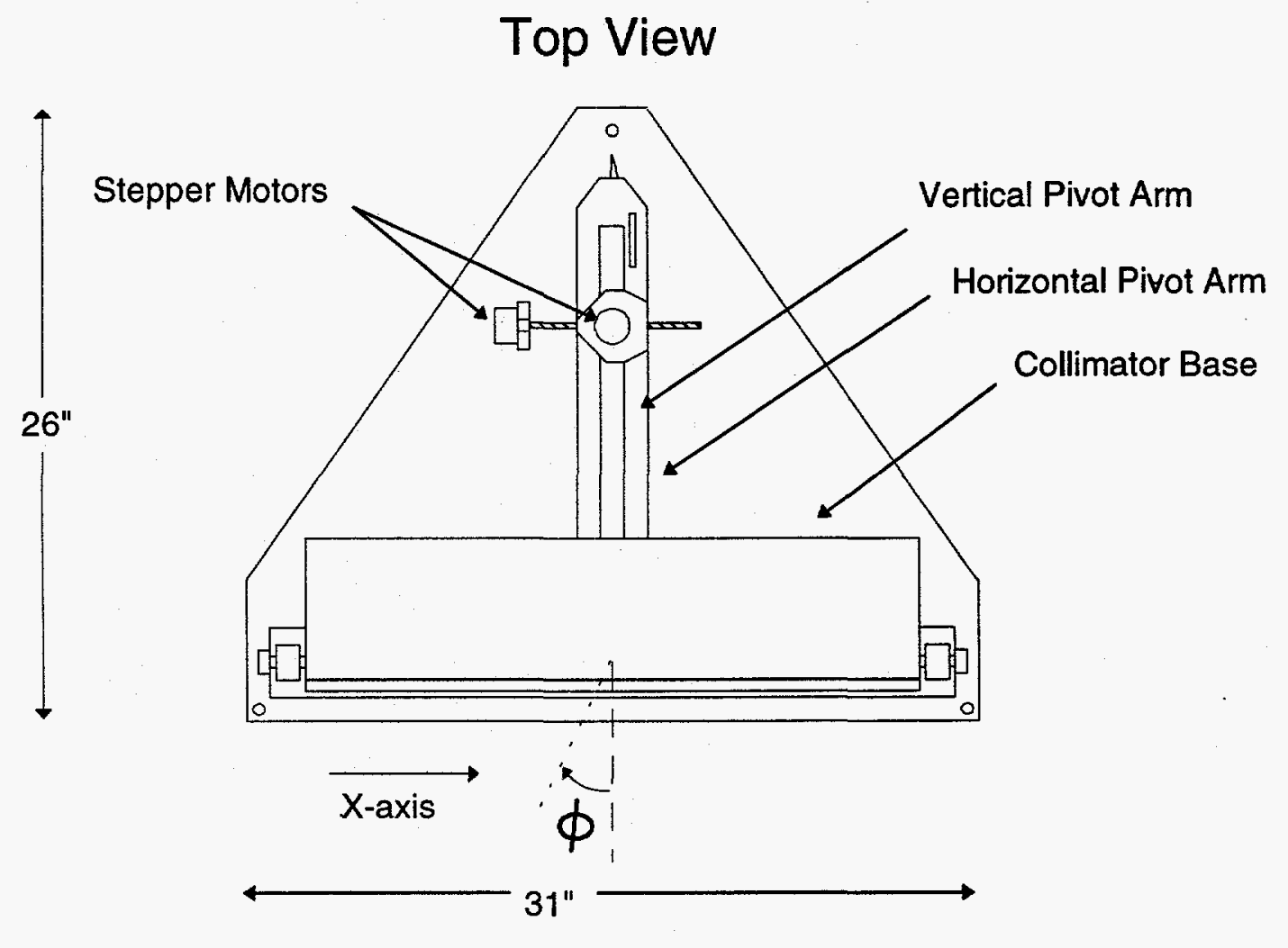

Side View

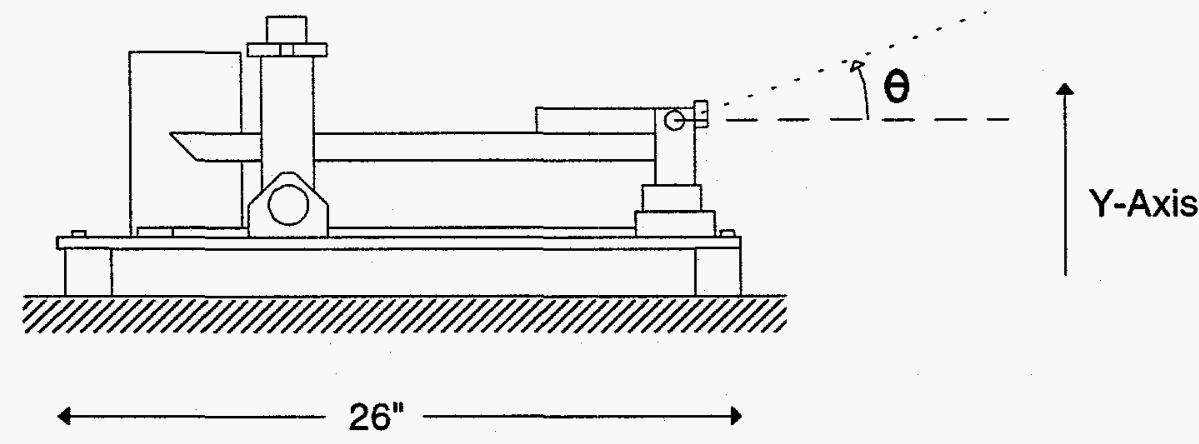

Fig. D.3 Geometry of the XRC goniometer. 
A triangular aluminum baseplate supported the two " $\mathrm{T}$ "-layers and an iron table in turn supported the baseplate's three legs. An adjustable length pipe connector formed each leg, allowing the leveling of the baseplate. To insure stability and position reproducibility, each leg was bolted to the iron table and the iron table was loaded at its base with over $500 \mathrm{lb}$. of lead bricks.

Two stepper motors controlled rotations in $\phi$ and $\theta$ by turning bolts through threaded fixtures on the moment arms of the top and bottom "T" layers. We chose the distance from the pivots to satisfy the simple conversion, 1 turn $=50$ steps $=0.2^{\circ}$.

To veto cosmic ray background, a 4-sided scintillator "box" was assembled to surround all exposed sides of the detector except for the collimator face. The scintillator from each side was cut and polished before epoxied together. Two phototubes were glued to the scintillator with translucent RTV, one along the y-axis and the other along the $\mathrm{x}$ axis as the axes are defined in Figure D.3. The scintillator was wrapped with aluminum foil to reflect scintillated light and then wrapped again with black mat board and black photographic tape seal against light leaks.

Managing all this detector apparatus was the electronics, the final component of the detector assembly. The electronics processed the signals from the detector components into actual X-ray counts and sent signals to the stepper motors to control the goniometer's movements. The PWC and the phototubes were the sources of input signals.

A pre-amplifier preprocessed charge collected on the PWC into a viable signal for the discriminator. The pre-amplifier was fabricated from a circuit consisting of two emitter-followers in series as shown in Figure D.4. The rise time of the output signal was 300 nsec.

The amplified analog signals from the PWC and the phototubes were then digitized by discriminators with a $10 \mathrm{~ns}$ rise time and a $-30 \mathrm{mV}$ threshold. A logic unit summed the discriminated phototube pulses and then a gate generator increased the width of the summed pulse to $250 \mu$ s to allow for any accidental double-counting in the PWC. 


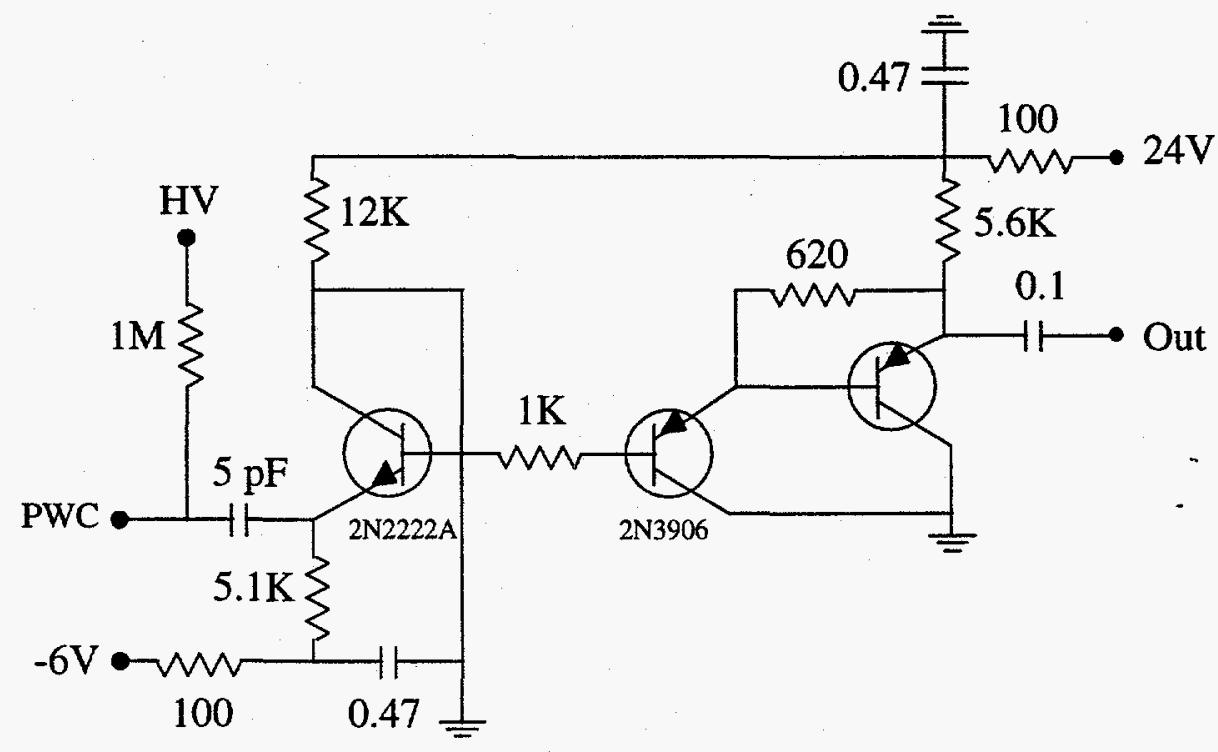

Fig. D.4 Circuit diagram of the pre-amplifier used in the XRC electronics. All units are in $\Omega$ or $\mu \mathrm{F}$ unless indicated otherwise.

Another gate generator narrowed the discriminated PWC pulse to $10 \mu$ s and time-delayed it to lie at the center of a scintillator pulse generated from the same event. This inhibited over-vetoing of cosmic rays due to any multiple misfiring in the chamber.

Finally, a logic unit used the gate generated pulses from the scintillator to veto any corresponding pulses from the PWC. Output from this logic unit translated into counts in a scalar reset and read through the parallel port of a PC. The electronics for counting $\mathrm{X}$-ray events as described here is summarized in Figure D.5.

To control the stepper motors, a simple driver circuit was assembled using LM18293 chips. The PC communicated commands to the circuit through its parallel port. A step commanded by the PC translated into 4 rotations of the motor windings or $0.004^{\circ}$ of the goniometer arms. A simple FORTRAN program enabled complete automation of the goniometer movements and data collection. 


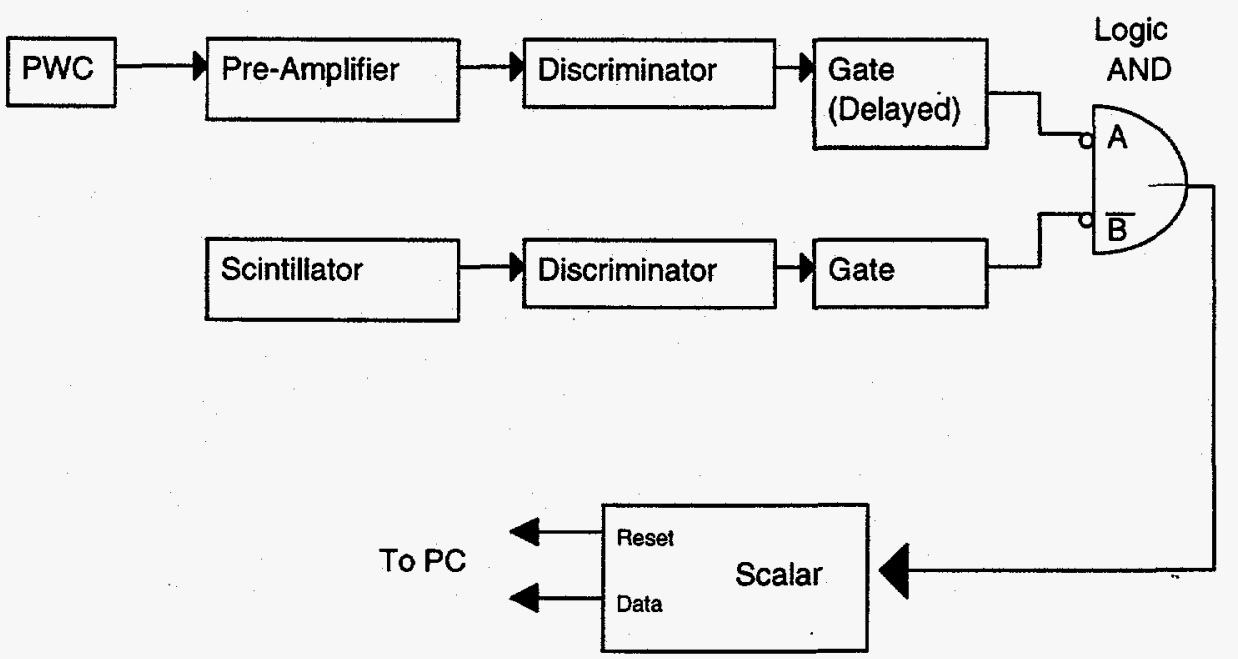

Fig. D.5 Flow chart for the XRC electronics.

\section{D.3 XRC Calibration}

Several source and detector assembly components of the XRC experiment were calibrated. The XRC cannon was aligned to minimize occultation losses of the X-ray beam. To define the collimator pointing axes relative to the beam direction, the goniometer arms were calibrated. Finally, the two phototubes and the PWC were plateaued.

\section{D.3.1 Alignment of the XRC Cannon}

Before the XRC cannon was placed into its support apparatus, the positions of the vertical support rods were determined by using a string suspended across the length of the pipe as a horizontal reference. To mark the position of each pipe hanger along the vertical rods, the string was pulled taut and the ends positioned to the same desired height.

Final vertical and horizontal alignments were made after the cannon was position in the pipe hangers. The vertical reference originated from a suspended bucket of water with a plastic tube attached at the bottom. The height of the water at the other end of the tube was used as a vertical reference. For the horizontal reference another string was tied taut to the pipe hangers at the ends of the cannon. An L-shaped ruler with a leveler was 
used to measure the distance between the pipe and the string. Any minor misalignments were then corrected by adjusting the nuts that held the two rods to an angle bracket.

The accuracy of the final alignment was limited by the poorest reference, the string that defined the horizontal reference. Repeated measurements at each support point along the cannon indicated that the resulting horizontal alignment was good to $\pm 1 / 8$ ".

\section{D.3.2 Calibration of the Goniometer}

The goniometer apparatus, shown in Figure D.3 rested on top of an iron table that was weighed down by $+500 \mathrm{lb}$. of lead bricks. The table was needed in order to bring the detector apparatus to the same height as the X-ray beam. Using two levelers, the baseplate of the goniometer apparatus was first leveled through its adjustable legs. When the adjustments were completed, the legs were then bolted to the iron table.

The zero angle positions of the horizontal and vertical arms were calibrated to the peak transmission of one of the collimator modules. To mark these positions, metal tips were glued to the end of the horizontal arm and to the vertical stepper motor's turning shaft. Each pointed to a fixed reference mark that defined the goniometer's rotation origin.

\section{D.3.3 Plateauing of the Detector}

The voltage setting on a detector unit is determined by increasing its voltage until its efficiency for detecting counts 'plateaus'. This is usually exhibited as a function of voltage by a rapid rise in the number of counts detected followed by a sharp 'knee' and then a plateau. A continued increase of the voltage along this plateau will eventually lead to a breakdown of the detector resulting in a runaway in the number of counts detected.

Three detector units were plateaud: two phototubes and one proportional wire chamber (PWC). The cosmic rays were used as the source to plateau the phototubes. Coincidencing the scintillator signals with the PWC, the resulting veto counts were accumulated as a function of the phototube voltage as shown in Figure D.6. The operating voltages were set to $2300 \mathrm{~V}$ and $2200 \mathrm{~V}$ for phototube 1 and 2, respectively. As defined in Figure D.3, phototubes 1 and 2 were oriented along the $x$-axis and $y$-axis respectively. 


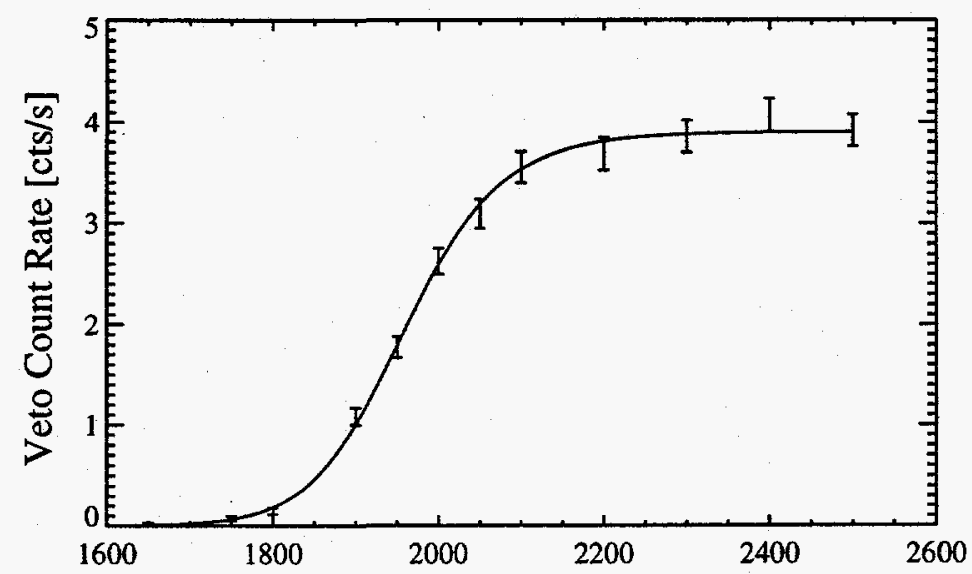

Phototube 1 Voltage [V]

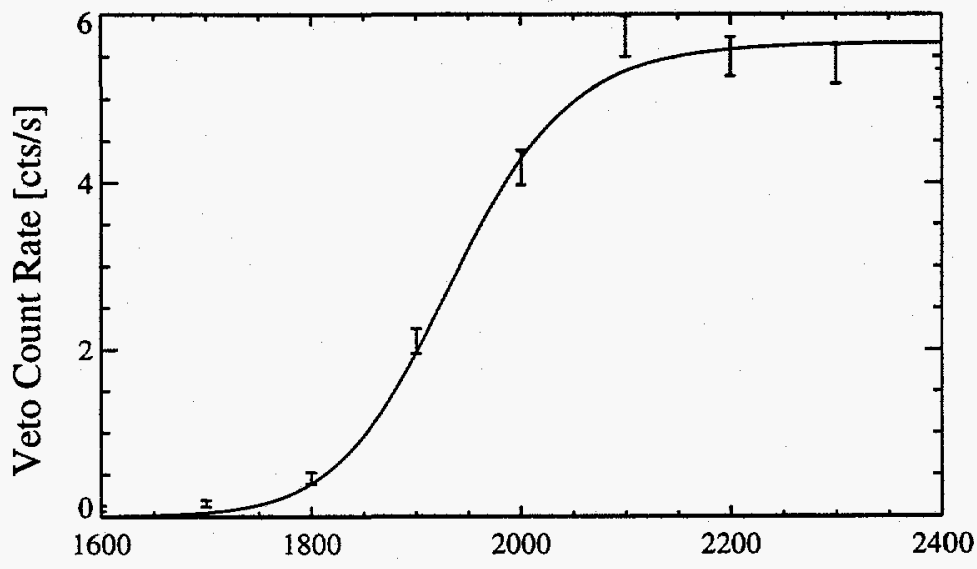

Phototube 2 Voltage [V]

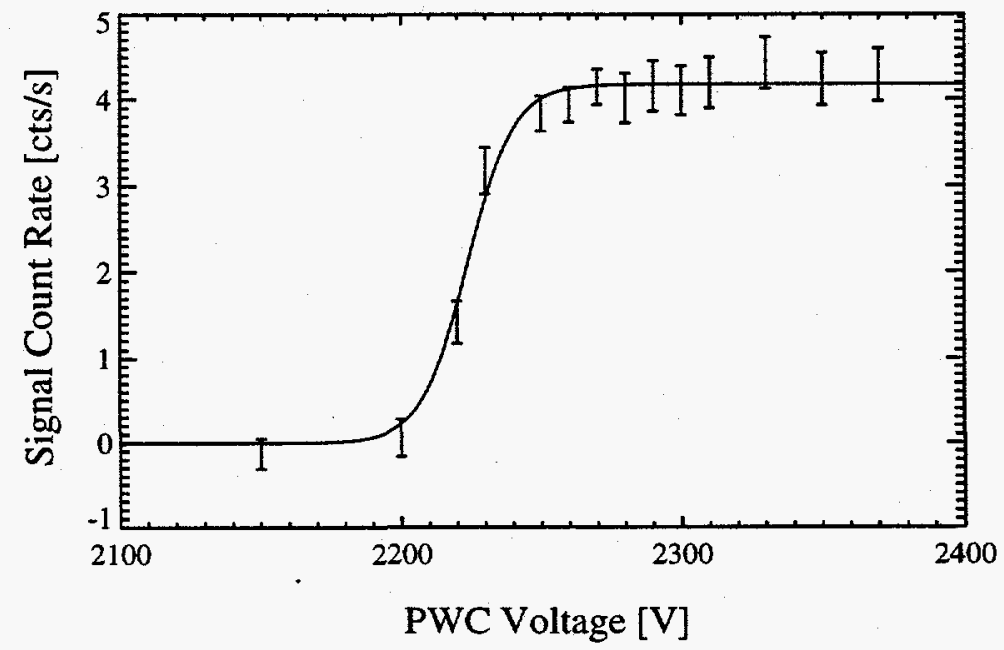

Fig. D.6 Plateau curves for phototubes 1 and 2 and PWC. The operating voltages were set to $2300 \mathrm{~V}$, $2200 \mathrm{~V}$ and $2300 \mathrm{~V}$, for phototubes 1 and 2 and PWC, respectively. 
The X-ray beam from the XRC cannon was used as the source to plateau the PWC. The cosmic rays were first vetoed by coincidencing the outputs of the PWC and the scintillator. In addition, to eliminate the effects from the residual isotope activity of the chamber (described later in Section D.4.1), the signal counts with no X-ray source was subtracted from the signal counts with the $\mathrm{X}$-ray source. The difference represented the "actual" detected source rate. From the resulting plateau curve for the PWC shown in Figure D.6, the operating voltage was set to $2300 \mathrm{~V}$.

\section{D.4 XRC Experiment}

The collimator response measurements began on September 9, 1993, and continued for 3 months. In that period of time, all 28 collimator modules were measured, ( 8 flight units and 20 spares). In addition, module 18 was re-measured several times to test the reproducibility of the detector apparatus.

\section{D.4.1 Backgrounds}

The veto system of the detector apparatus was successful in identifying coincidence events with rate of $\sim 3 \mathrm{cts} \mathrm{s}^{-1}$. These events are due to cosmic rays. At sea level, the total flux from cosmic rays crossing a unit horizontal area from above is estimated at $0.02 \mathrm{~cm}^{-2} \mathrm{~s}^{-1}$. The PWC was on its side facing the X-ray beam, exposing a surface area of $177 \mathrm{~cm}^{2}$. Therefore, the expected rate of cosmic rays was $3.5 \mathrm{cts} \mathrm{s}^{-1}$.

After the veto counts were removed, a residual PWC count rate of $\sim 4 \mathrm{cts} \mathrm{s}^{-1}$ remained. The output pulses of the pre-amplifier and the phototubes were observed on a scope to verify that these residual counts were not caused by double-pulsing in the chamber nor due to incorrect time delays between the PWC and the scintillator signals. To insure that this residual rate was not due to unvetoed cosmic four sides of the detector were covered by scintillator material with two phototubes attached. Evidence indicates that the source of these residual counts originates from within the PWC, probably from radioactive impurities in the chamber's aluminum walls. 


\section{D.4.2 X-Ray Beam Intensity}

In designing the source assembly, the expected rate of X-rays detected by the PWC was estimated to insure that the resulting beam would be useful. The scattering and adsorption through the various materials and physical obstructions (e.g. the cannon's solid angle) were considered and are summarized in Table D.1. For a ${ }^{55} \mathrm{Fe}$ source with 25 $\mathrm{mC}$ activity the expected count rate in the PWC is $4.5 \mathrm{cts} \mathrm{s}^{-1}$.

To measure this rate, the goniometer was set to the peak of a collimator response $\left(0^{\circ}, 0^{\circ}\right)$ and counts were collected in both the presence and the absence of the X-ray beam. The latter measurement was needed to subtract the residual background discussed in the previous section. The difference of these two measurements yielded an observed count rate of $4.29 \pm 0.02 \mathrm{cts} \mathrm{s}^{-1}$.

The small discrepancy between the observed and expected count rate may be attributed to a smaller transmission through the collimator face than dictated by an idealized geometry. While the amount of area covered by the collimator material over its face accounts for only $2 \%$ of the total area, the effective area is slightly larger due to small imperfections and misalignments between different hexagonal cells.

To measure this effect, counts were collected again at $\left(0^{\circ}, 0^{\circ}\right)$ in both the presence and the absence of the X-ray beam with the collimator module removed from the detector apparatus. The difference of these two measurements yielded an observed count rate of $4.6 \pm 0.1 \mathrm{cts} \mathrm{s}^{-1}$. The ratio between the rates with and without the collimator in the apparatus gives $93 \pm 2 \%$ as the transmission through the collimator, compared to $98 \%$ due to the collimator's surface area alone. Taking into account this measured transmission through the collimator, the expected count rate is $4.27 \mathrm{cts} \mathrm{s}^{-1}$ which agrees with the observed rate within $1 \sigma$. 


\begin{tabular}{|l|r|}
\hline & Transmission \\
\hline Transmission through the pipe (Air, $200 \mu \mathrm{Hg})$ & 0.98 \\
\hline Transmission through the pipe window (5 mil mylar) & 0.75 \\
\hline Transmission through gap between pipe window and PWC (Air, 1 atm) & 0.70 \\
\hline Transmission through PWC window (1/2 mil aluminized mylar) & 0.97 \\
\hline Absorption efficiency of PWC gas (P10 gas, $90 \%$ Ar, 10\% $\left.\mathrm{CH}_{4}\right)$ & 0.97 \\
\hline Fraction of Source Area (3.75" $\left.\times 3.75^{\prime}\right)$ within pipe cross section & 0.97 \\
\hline Fraction of solid angle unobstructed by pipe ( $\pm 1 / 8^{\prime \prime}$ misalignment ) & 0.50 \\
\hline Fraction of collimator face area unobstructed by collimator material & $7.85 \times 10^{-8}$ \\
\hline Total Transmission & $4.85 \times 10^{-9}$ \\
\hline
\end{tabular}

\begin{tabular}{|l|r|}
\hline & $\begin{array}{c}\text { Count Rate } \\
{\left[\text { cts s }^{-1}\right]}\end{array}$ \\
\hline $\begin{array}{l}\text { Source: } 55 \mathrm{Fe}, \text { Activity: } 25.0 \mathrm{mC}, \text { Date: } 9-1-93 \\
\text { Spectrum: K } \alpha \text { fraction }=0.26, \text { Energy }=5.9 \mathrm{keV}\end{array}$ & $9.25 \times 10^{8}$ \\
\hline Expected Rate observed by PWC & 4.48 \\
\hline Measured Rate observed by PWC & $4.29 \pm 0.02$ \\
\hline
\end{tabular}

Table D.1 Summary of the transmissions through the various materials and obstructions and the expected and measured count rates in the PWC.

\section{D.4.3 Measurement Run}

The procedure for measuring the response function for a collimator module began with placing the unit in an aluminum frame and bolting the frame to the PWC with a $\frac{1}{2}$ mil mylar window in between to contain the gas. The detector was then positioned on the goniometer base. Since the X-ray beam covered only $\sim \frac{1}{3}$ of the collimator face, three positions were defined along the goniometer base such that the beam was incident at the center or near either end of a collimator face. Holes were drilled at these positions and an aluminum peg filled one of them to define the reference edge for the collimator module. 


\begin{tabular}{|l|c|c|c|c|c|}
\hline Name & $\begin{array}{c}\phi \text { Range } \\
{[\text { degrees] }}\end{array}$ & $\begin{array}{c}\theta \text { Range } \\
{[\text { degrees] }}\end{array}$ & $\begin{array}{c}\text { Step Size } \\
{[\text { degrees] }}\end{array}$ & $\begin{array}{c}\text { No. of } \\
\text { points }\end{array}$ & $\begin{array}{c}\text { Total time } \\
{[\mathrm{hrs}]}\end{array}$ \\
\hline Origin & $0^{\circ}$ & $0^{\circ}$ & $0.0^{\circ}$ & 1 & 0.1 \\
\hline Horizontal Scan & {$\left[-2.4^{\circ}, 2.4^{\circ}\right]$} & $0^{\circ}$ & $0.1^{\circ}$ & 49 & 6.2 \\
\hline Origin & $0^{\circ}$ & $0^{\circ}$ & $0.0^{\circ}$ & 1 & 6.4 \\
\hline Vertical Scan & $0^{\circ}$ & {$\left[-2.4^{\circ}, 2.4^{\circ}\right]$} & $0.1^{\circ}$ & 49 & 12.5 \\
\hline Origin & $0^{\circ}$ & $0^{\circ}$ & $0.0^{\circ}$ & 1 & 12.6 \\
\hline Coarse Grid & {$\left[-0.6^{\circ}, 0.6^{\circ}\right]$} & {$\left[-0.6^{\circ}, 0.6^{\circ}\right]$} & $0.2^{\circ}$ & 49 & 18.7 \\
\hline Background & $2.4^{\circ}$ & $2.4^{\circ}$ & $0.0^{\circ}$ & 1 & 18.9 \\
\hline Fine Grid & {$\left[-0.2^{\circ}, 0.2^{\circ}\right]$} & {$\left[-0.2^{\circ}, 0.2^{\circ}\right]$} & $0.1^{\circ}$ & 25 & 22.0 \\
\hline Background & $2.4^{\circ}$ & $2.4^{\circ}$ & $0.0^{\circ}$ & 1 & 22.1 \\
\hline Origin & $0^{\circ}$ & $0^{\circ}$ & $0.0^{\circ}$ & 1 & 22.2 \\
\hline
\end{tabular}

Table D.2 List of measurements made for each run.

For each collimator, this position was chosen randomly.

Once the detector was fully assembled on the goniometer, the PWC was flushed with P10 gas for $\sim 20$ minutes to remove air in the chamber. In addition, once the measurements began, a pressure regulator insured that a steady stream of P10 gas flowed through the chamber $\left(\sim 1 \mathrm{~cm}^{3} \mathrm{~s}^{-1}\right)$ to flush out any air that may have leaked in.

The measurements of X-rays at different angles relative to the beam axis was fully automated. Commands were read sequentially from a file and sent to the stepper motor through a serial port on a PC. As listed on Table D.2, the measurement run consisted of a horizontal scan in $\phi$, a vertical scan in $\theta$, a coarse $1.2^{\circ} \times 1.2^{\circ}$ grid and a fine $0.4^{\circ} \times 0.4^{\circ}$ grid. Counts were collected at each angular position for 7.5 minutes. Each measurement run lasted 22 hours. 


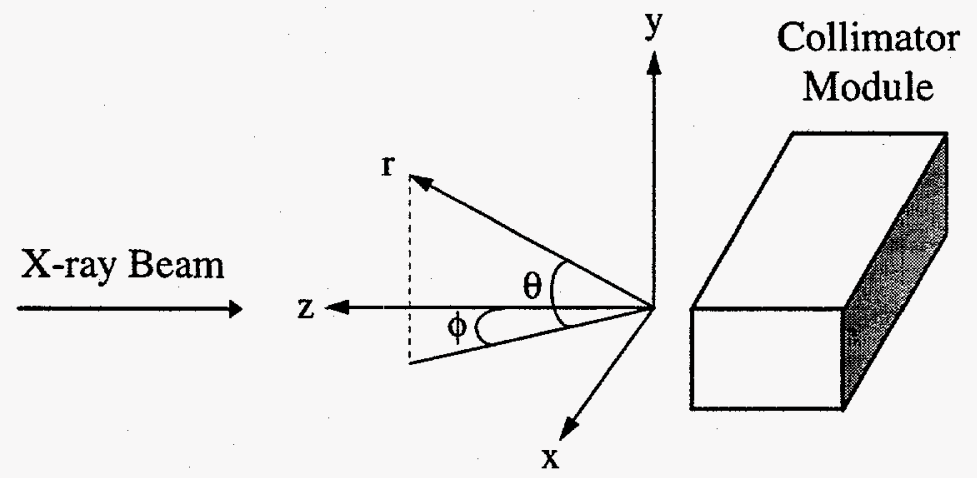

Fig. D.7 Definition of $\phi$ and $\theta$ in the xyz-coordinate system. The X-ray beam lies along the $-\mathrm{z}$-axis. At $\phi=\theta=0^{\circ}$ the collimator module rests on the $x z$-plane with its collimator face is parallel to the xy-plane. The shaded region indicates the rear collimator face.

\section{D.5 XRC Data Analysis}

\section{D.5.1 Coordinate Systems}

In this experiment, three rotational coordinate systems are identified: one from the goniometer and two from the collimator module. The first is defined by the goniometer arms. When the arms are rotated to its origin, the direction the arms are pointing defines the goniometer g-axis. This axis will lie along the z-direction of the goniometer coordinate system.

For a perfect collimator module, all the hexagonal tubes are aligned and are parallel to the bridge sheets that support them. The direction that the tubes are pointing towards (i.e. peak of the response function) defines what will be referred to as the $z$-axis. This is the inertial coordinate system of a perfect collimator module. In terms of an actual module, this coordinate system is defined by the collimator bridge sheets.

The third coordinate system is that of the actual collimator module. Here, small misalignments of the hexagonal tubes may cause the effective pointing direction to be offset from the z-axis. The effective direction that the actual collimator module is pointing towards is identified as the $c$-axis. This axis defines the z-direction of the body coordinate system. 
The angles referred to in the text as $\phi$ and $\theta$ are illustrated in Figure D.7. For a given coordinate system, a unit vector $\mathbf{r}$ is defined in terms of these two angles as

$$
\mathbf{r}=\cos \theta \sin \phi \mathbf{x}+\sin \theta \mathbf{y}+\cos \theta \cos \phi \mathbf{z}
$$

where $\mathbf{x}, \mathbf{y}, \mathbf{z}$ are defined relative to one of the three coordinate systems discussed.

\section{D.5.2 Method for Determining Collimator Response Characteristics}

The data from each measurement run was analyzed to determine the center and width (FWHM) of the response function for that collimator module. An elliptical cone was chosen as the functional form to interpolate the 2 variables that determine the collimator module characteristics relevant to USA performance, acceptance in $\phi$ and $\theta$. Its expression is given by

$$
f_{s}(\phi, \theta, t)=-m \sqrt{\left(\phi-\phi_{0}\right)^{2}+a^{2}\left(\theta-\theta_{0}\right)^{2}}+b
$$

where $\phi_{0}, \theta_{0}, m, a$ and $b$ are fit parameters. $\left(\phi_{0}, \theta_{0}\right)$ defines the center, $b / m$ defines the FWHM along $\phi$ (horizontal axis) and $b /(m a)$ defines the FWHM along $\theta$ (vertical axis). [D.2] is constrained to points within the ellipse

$$
\left(\phi-\phi_{0}\right)^{2}+a^{2}\left(\theta-\theta_{0}\right)^{2}=(b / m)^{2}
$$

where $f_{S}(\phi, \theta, t)>0$. For all points outside this ellipse, $f_{S} \equiv 0$.

In addition to the signal from the $\mathrm{X}$-ray beam, a residual background contributed to the overall observed count rate. As discussed in Section D.5.1, this background was probably due to impurities in the proportional chamber aluminum walls. To account for this systematic offset the background was modeled by a polynomial in time,

$$
f_{B}(t)=\sum_{j=0}^{N} c_{j} t^{j}
$$




\begin{tabular}{|c|c|c|c|c|}
\hline \multirow{2}{*}{ Run } & \multicolumn{2}{|c|}{ Response Function Centers } & \multicolumn{2}{c|}{ Response Function FWHM } \\
\cline { 2 - 5 } & $\begin{array}{c}\phi \text { (g-axis) } \\
{\left[ \pm 0.005^{\circ}\right]}\end{array}$ & $\begin{array}{c}\theta \text { (g-axis) } \\
{\left[ \pm 0.004^{\circ}\right]}\end{array}$ & $\begin{array}{c}\phi \\
{\left[ \pm 0.02^{\circ}\right]}\end{array}$ & $\begin{array}{c}\theta \\
{\left[ \pm 0.02^{\circ}\right]}\end{array}$ \\
\hline 1 & $0.057^{\circ}$ & $0.135^{\circ}$ & $1.21^{\circ}$ & $1.19^{\circ}$ \\
\hline 2 & $0.045^{\circ}$ & $0.124^{\circ}$ & $1.20^{\circ}$ & $1.20^{\circ}$ \\
\hline 3 & $0.048^{\circ}$ & $0.128^{\circ}$ & $1.27^{\circ}$ & $1.20^{\circ}$ \\
\hline 4 & $0.049^{\circ}$ & $0.123^{\circ}$ & $1.23^{\circ}$ & $1.19^{\circ}$ \\
\hline 5 & $0.048^{\circ}$ & $0.125^{\circ}$ & $1.22^{\circ}$ & $1.20^{\circ}$ \\
\hline
\end{tabular}

\begin{tabular}{|c|c|c|c|c|}
\hline Average & $0.049^{\circ}$ & $0.125^{\circ}$ & $1.23^{\circ}$ & $1.196^{\circ}$ \\
\hline$\sqrt{\text { Variance }}$ & $0.005^{\circ}$ & $0.005^{\circ}$ & $0.03^{\circ}$ & $0.005^{\circ}$ \\
\hline
\end{tabular}

Table D.3 Summary of the fit results to five separate measurement runs with Collimator 18. The response function centers are angles relative to the goniometer axis (g-axis). The overall variance for each parameter is consistent with its statistical uncertainty for each run.

The sum of [D.2] and [D.4] was applied to the fit of the entire data set in a measurement run. The MINUIT89 program $^{1}$ was employed to minimize the $\chi^{2}$ of the parameters in the fit using the SIMPLEX algorithm. The degree of the polynomial in [D.4] was varied from 0 to 4 and the fit with the smallest $\chi^{2}$ per degree of freedom, $v$ was selected.

\section{D.5.3 Accuracy of the Detector Apparatus}

To make a meaningful comparison of the centers from different modules, the detector apparatus must be able to consistently reproduce the same response function for repeated measurements of a given module. Several measurement runs were performed on collimator module 18. The data from each run was fit for its response function center and width using the method described in the previous section. The results of these fits, summarized in Table D.3, show that the overall variance of the centers and widths are

\footnotetext{
${ }^{1}$ Application Software Group (1992), Minuit, Function Minimization and Error Analysis, CERN Program Library Entry D506, CERN Geneva, Switzerland.
} 
consistent with the statistical uncertainty in determining each parameter. Namely, the detector apparatus produces reproducible measurements within the resolution of counting statistics. The accuracy of the detector apparatus is estimated conservatively to be $0.005^{\circ}$

\section{D.5.4 Determination of the Goniometer Axis}

All of the response functions were measured at angles relative to the g-axis of the goniometer coordinate system. However, the g-axis itself may be rotated from the true collimator $z$-axis. This true $z$-axis defines the collimator's inertial coordinate system, described in Section D.5.1.

The center of a response function is the location of its peak. This response function center is quantified by parameters $\phi_{0}$ and $\theta_{0}$ of the elliptical cone in [D.2]. The goniometer g-axis can be expressed in the inertial coordinate system in terms of these fitted parameters. The response function centers can then be transformed into the inertial coordinate system to determine the absolute misalignment of a collimator module.

Assume the goniometer g-axis is rotated from the inertial coordinate system by 3 small Euler angles, $\varepsilon_{1}, \varepsilon_{2}$ and $\varepsilon_{3}$. The rotation matrix to second order in these angles is

$$
\left(\begin{array}{ccc}
1-\frac{1}{2}\left(\varepsilon_{1}+\varepsilon_{3}\right)^{2} & \varepsilon_{1}+\varepsilon_{3} & \varepsilon_{2} \varepsilon_{3} \\
-\left(\varepsilon_{1}+\varepsilon_{3}\right) & 1-\frac{1}{2}\left(\varepsilon_{1}+\varepsilon_{3}\right)^{2}-\frac{1}{2} \varepsilon_{2}^{2} & \varepsilon_{2} \\
\varepsilon_{1} \varepsilon_{2} & -\varepsilon_{2} & 1-\frac{1}{2} \varepsilon_{2}{ }^{2}
\end{array}\right)
$$

which gives the coordinates of the g-axis in terms of the z-axis coordinate system as

$$
\begin{gathered}
g=x_{g}+y_{g}+z_{g} \\
\mathbf{x}_{\mathbf{g}}=\left(\begin{array}{c}
1-\frac{1}{2}\left(\varepsilon_{1}+\varepsilon_{3}\right)^{2} \\
-\left(\varepsilon_{1}+\varepsilon_{3}\right) \\
\varepsilon_{1} \varepsilon_{2}
\end{array}\right), \mathbf{y}_{\mathbf{g}}=\left(\begin{array}{c}
\varepsilon_{1}+\varepsilon_{3} \\
1-\frac{1}{2}\left(\varepsilon_{1}+\varepsilon_{3}\right)^{2}-\frac{1}{2} \varepsilon_{2}{ }^{2} \\
-\varepsilon_{2}
\end{array}\right), \mathbf{z}_{\mathbf{g}}=\left(\begin{array}{c}
\varepsilon_{2} \varepsilon_{3} \\
\varepsilon_{2} \\
1-\frac{1}{2} \varepsilon_{2}{ }^{2}
\end{array}\right)
\end{gathered}
$$

For the collimator c-axis, let $(\phi, \theta)$ denote its direction with respect to the inertial coordinate system and $\left(\phi_{\mathrm{g}}, \theta_{\mathrm{g}}\right)$ its direction with respect to the goniometer coordinate 


\begin{tabular}{|c|c|}
\hline & Value $\left[ \pm 0.005^{\circ}\right]$ \\
\hline$\varepsilon_{1}$ & $-0.117^{\circ}$ \\
\hline$\varepsilon_{2}$ & $-0.104^{\circ}$ \\
\hline$\varepsilon_{3}$ & $-0.183^{\circ}$ \\
\hline
\end{tabular}

Table D.4 Euler angles needed to rotate goniometer axis to the direction observed from the inertial z-axis.

system. The c-axis may be expressed in terms of the these two coordinate systems as

$$
\begin{aligned}
\mathbf{c} & =\cos \theta \sin \phi \mathbf{x}+\sin \theta \mathbf{y}+\cos \theta \cos \phi \mathbf{z} \\
& =\cos \theta_{g} \sin \phi_{g} \mathbf{x}_{\mathbf{g}}+\sin \theta_{g} \mathbf{y}_{\mathbf{g}}+\cos \theta_{g} \cos \phi_{g} \mathbf{z}_{\mathbf{g}}
\end{aligned}
$$

Replacing the goniometer unit vectors $\mathbf{x}_{\mathrm{g}}, \mathbf{y}_{\mathrm{g}}, \mathbf{z}_{\mathrm{g}}$ by their representation in the inertial coordinate system [D.6], and then equating the components for each of the unit vectors $\mathbf{x}, \mathbf{y}, \mathbf{z}$ gives $(\phi, \theta)$ in terms of $\left(\phi_{\mathrm{g}}, \theta_{\mathrm{g}}\right)$ and the Euler angles $\varepsilon_{1}, \varepsilon_{2}, \varepsilon_{3}$ to second order as

$$
\begin{aligned}
& \phi \approx \phi_{g}+\theta_{g}\left(\varepsilon_{1}+\varepsilon_{3}\right)+\varepsilon_{2} \varepsilon_{3} \\
& \theta \approx \theta_{g}-\phi_{g}\left(\varepsilon_{1}+\varepsilon_{3}\right)+\varepsilon_{2} \\
& \theta^{2}+\phi^{2} \approx \phi_{g}^{2}+\left(\theta_{g}+\varepsilon_{2}\right)^{2}
\end{aligned}
$$

The three equations in [D.8] can solve three of the 5 unknowns $\left(\phi, \theta, \varepsilon_{1}, \varepsilon_{2}, \varepsilon_{3}\right)$ for a given collimator $\mathrm{c}$-axis. To solve for the remaining parameters, perform a $\pi$-rotation of the collimator module about the $\mathrm{x}$-axis of the inertial coordinate system.

$$
\mathbf{c}^{\prime}=\left(\begin{array}{ccc}
1 & 0 & 0 \\
0 & -1 & 0 \\
0 & 0 & -1
\end{array}\right)\left(\begin{array}{c}
\cos \theta \sin \phi \\
\sin \theta \\
\cos \theta \cos \phi
\end{array}\right)=\left(\begin{array}{c}
\cos \theta \sin \phi \\
-\sin \theta \\
-\cos \theta \cos \phi
\end{array}\right)
$$

Namely, $\phi \rightarrow-\phi$ and $\theta \rightarrow \theta+\pi$. Another measurement of the collimator $c$-axis in the goniometer coordinate system gives $\left(\phi_{g}^{\prime}, \theta_{g}^{\prime}\right)$. This procedure amounts to replacing $\phi_{g} \rightarrow \phi_{g}^{\prime}, \theta_{g} \rightarrow \theta_{g}^{\prime}, \theta \rightarrow-\theta$ in the first two equations of [D.8] which gives the two additional equations needed to solve all of the 5 unknowns for a given collimator $\mathrm{c}$-axis. 
To actually perform this measurement, the collimator module is flipped $180^{\circ}$ about its long $\mathrm{x}$-axis, the collimator response function is re-measured and then the center is determined using the fitting algorithm described in Section D.5.2. Table D.4 shows the values for the Euler angles obtained using this procedure. Using these values in [D.8] and setting $\phi_{g}=\theta_{g}=0$ gives for the goniometer axis,

$$
(\phi, \theta)_{\mathrm{g}-a x i s}=\left(0.019^{\circ},-0.104^{\circ}\right)\left[ \pm 0.005^{\circ}\right]
$$

\section{D.5.5 Monte Carlo Simulation}

Simulations of the response functions for each module are used to estimate the total response function expected by combining two or more of the modules. The simulation is performed in two stages. In the first stage, photons are generated at uniform random positions along a square region and emitted at uniform random solid angles. The photon is projected down the length of the XRC cannon and if it lies within the pipe's effective radius, then the photon is permitted to enter the collimator face.

The second stage projects each photon through the collimator. The collimator axis is first rotated to the direction of the response function center for the collimator module being simulated. If a photon crosses one or more hexagonal cell boundaries as it traverses the depth of the collimator, then that event is discarded. The simulation is repeated using the same angles as in the experiment measurement run to generate simulated data.

\section{D.6 XRC Results}

\section{D.6.1 Characterization of the Collimator Modules}

For each of the 28 collimator modules constructed, the center and FWHM of its response function were determined using the fitting technique described in the previous section. Table D.5 summarizes the results of these fits, where the response function centers listed have been corrected by [D.8] so that they are relative to the inertial 
coordinate system. In the inertial coordinate system, the average offset of the response function centers is observed to be

$$
(\langle\phi\rangle,\langle\theta\rangle)=\left(0.036^{\circ},-0.015^{\circ}\right)\left[ \pm 0.001^{\circ}\right]
$$

with a $\sqrt{\text { variance }}$ or spread of the centers as

$$
(\phi, \theta)_{\sigma}=\left(0.03^{\circ}, 0.06^{\circ}\right)
$$

The overall widths of the response functions are

$$
(\phi, \theta)_{F W H M}=\left(1.237^{\circ}, 1.185^{\circ}\right)\left[ \pm 0.004^{\circ}\right]
$$

with a relatively smaller $\sqrt{\text { variance }}$ as

$$
(\phi, \theta)_{\sigma(F W H M)}=\left(0.02^{\circ}, 0.02^{\circ}\right)
$$

An additional fit was performed on each module using the results from the simulations of its response function. The elliptical cone used to fit the center and width of each response function was replaced with the simulated data. The background was then fit as a polynomial in time for degrees from $0-4$. The fit giving the smallest $\chi^{2} / v$ was selected and its corresponding confidence level is displayed in the last column of Table D.5.

The results of this additional fit are also shown at the end of this Appendix. There, the data from the $\phi$-scan and the $\theta$-scan for each module are displayed along with overplots of the fits using the simulated data. The difference between the data and the fit is displayed below each scan.

\section{D.6.2 Final Selection of Collimator Modules}

Twenty-four modules are selected to construct three full collimators ( 2 flight, 1 spare). The final selection of modules is shown in Table D.6. The selection of modules and their orientations $\phi=(0$ or $\pi)$ in the collimator frame was based on several criteria. Modules with the larger confidence levels $(>1 \%)$ to the fit to the simulations of the 


\begin{tabular}{|c|c|c|c|c|c|c|c|c|c|}
\hline \multirow[t]{2}{*}{ Module } & \multicolumn{4}{|c|}{ Center of Response Functions } & \multicolumn{2}{|c|}{ FWHM } & Cone & \multicolumn{2}{|c|}{ MC } \\
\hline & $\begin{array}{c}\phi \\
\pm\left[0.005^{\circ}\right] \\
\text { (g-axis) }\end{array}$ & $\begin{array}{c}\theta \\
\pm\left[0.004^{\circ}\right] \\
(g \text {-axis })\end{array}$ & $\begin{array}{c}\phi \\
\pm\left[0.005^{\circ}\right] \\
(z \text {-axis })\end{array}$ & $\begin{array}{c}\theta \\
\pm\left[0.004^{\circ}\right] \\
(z \text {-axis })\end{array}$ & $\begin{array}{c}\phi \\
\pm\left[0.02^{\circ}\right]\end{array}$ & $\begin{array}{c}\theta \\
\pm\left[0.02^{\circ}\right]\end{array}$ & $\chi^{2 / v}$ & $\chi^{2 / v}$ & $\mathrm{CL}(\%)$ \\
\hline 1 & 0.070 & 0.016 & 0.084 & -0.067 & 1.25 & 1.17 & 1.40 & 1.12 & 15 \\
\hline 2 & 0.019 & 0.042 & 0.025 & -0.056 & 1.24 & 1.20 & 1.41 & 1.00 & 49 \\
\hline 3 & 0.037 & 0.106 & 0.024 & 0.014 & 1.24 & 1.16 & 1.53 & 1.29 & 0.6 \\
\hline 4 & -0.015 & 0.054 & -0.012 & -0.054 & 1.23 & 1.19 & 1.55 & 1.32 & 0.3 \\
\hline 5 & 0.037 & 0.175 & 0.004 & 0.083 & 1.24 & 1.16 & 1.51 & 1.19 & 4 \\
\hline 6 & 0.068 & 0.131 & 0.048 & 0.048 & 1.21 & 1.19 & 1.58 & 1.31 & 0.3 \\
\hline 7 & 0.010 & 0.040 & 0.017 & -0.061 & 1.26 & 1.17 & 1.44 & 1.40 & 0.03 \\
\hline 8 & 0.021 & 0.130 & 0.001 & 0.033 & 1.25 & 1.20 & 1.26 & 1.12 & 14 \\
\hline 9 & 0.012 & 0.140 & -0.011 & 0.040 & 1.25 & 1.19 & 1.70 & 1.42 & 0.02 \\
\hline 10 & -0.007 & 0.038 & 0.001 & -0.068 & 1.19 & 1.19 & 1.37 & 1.21 & 3 \\
\hline 11] & 0.038 & 0.046 & 0.043 & -0.046 & 1.20 & 1.20 & 1.66 & 1.30 & 0.4 \\
\hline 12 & 0.031 & 0.111 & 0.017 & 0.017 & 1.28 & 1.18 & 1.46 & 1.44 & 0.01 \\
\hline 13 & 0.037 & 0.088 & 0.030 & -0.004 & 1.23 & 1.20 & 1.66 & 1.31 & 0.4 \\
\hline 14 & 0.043 & 0.191 & 0.005 & 0.100 & 1.28 & 1.17 & 1.50 & 1.30 & 0.5 \\
\hline 15 & 0.073 & 0.174 & 0.040 & 0.092 & 1.25 & 1.19 & 1.47 & 1.17 & 6 \\
\hline 16 & 0.048 & 0.044 & 0.054 & -0.045 & 1.21 & 1.20 & 1.56 & 1.22 & 2 \\
\hline 17 & 0.063 & 0.089 & 0.055 & 0.004 & 1.22 & 1.18 & 1.73 & 1.37 & 0.08 \\
\hline 18 & 0.057 & 0.135 & 0.036 & 0.049 & 1.21 & 1.19 & 1.31 & 1.10 & 18 \\
\hline 19 & 0.100 & 0.053 & 0.103 & -0.021 & 1.23 & 1.20 & 1.17 & 0.94 & 72 \\
\hline 20 & 0.084 & 0.065 & 0.083 & -0.013 & 1.25 & 1.19 & 1.30 & 1.15 & 8 \\
\hline 21 & 0.040 & 0.073 & 0.037 & -0.019 & 1.22 & 1.14 & 1.43 & 1.35 & 0.1 \\
\hline 22 & 0.017 & 0.028 & 0.028 & -0.070 & 1.23 & 1.19 & 1.34 & 1.16 & 7 \\
\hline 23 & 0.045 & 0.085 & 0.038 & -0.005 & 1.27 & 1.21 & 1.47 & 1.30 & 0.5 \\
\hline 24 & 0.046 & -0.042 & 0.078 & -0.132 & 1.23 & 1.18 & 1.25 & 1.11 & 16 \\
\hline 25 & 0.024 & 0.032 & 0.033 & -0.064 & 1.23 & 1.19 & 1.33 & 1.21 & 3 \\
\hline 26 & 0.008 & 0.058 & 0.010 & -0.043 & 1.27 & 1.19 & 1.37 & 1.27 & 0.8 \\
\hline 27 & 0.052 & 0.006 & 0.069 & -0.082 & 1.23 & 1.17 & 1.04 & 0.99 & 54 \\
\hline 28 & 0.016 & 0.034 & 0.025 & -0.065 & 1.24 & 1.18 & 1.54 & 1.50 & 0.001 \\
\hline
\end{tabular}

\begin{tabular}{|l|l|l|l|l|l|l|}
\hline Avg. & 0.04 & 0.08 & 0.03 & -0.02 & 1.24 & 1.18 \\
\hline$\sigma$ & 0.03 & 0.06 & 0.03 & 0.06 & 0.02 & 0.02 \\
\hline
\end{tabular}

Table D.5 Characterization of all the collimator modules by fitting an elliptical cone to the data (columns 2-8). The centers of the response functions are given relative to the goniometer coordinate system (columns 2-3) and the inertial coordinate system (columns 4-5). Monte Carlo simulation of each module with a background polynomial in time was also fit to data. The resulting $\chi^{2} / v$ and confidence levels are given in the last two columns. The last two rows represent the average and the $\sqrt{\text { variance }}$ of the fitted quantities in columns 1-4. 


\begin{tabular}{|c|c|c|c|c|}
\hline Collimator & Segment & Module & Relative Rotation & Status \\
\hline \multirow{8}{*}{$\mathrm{C}-1$} & & 5 & $0^{\circ}$ & \multirow{8}{*}{$\begin{array}{c}\text { Flight } \\
\text { Collimator }\end{array}$} \\
\hline & 1 & 20 & $180^{\circ}$ & \\
\hline & & 8 & $0^{\circ}$ & \\
\hline & & 14 & $180^{\circ}$ & \\
\hline & \multirow{4}{*}{2} & 1 & $180^{\circ}$ & \\
\hline & & 7 & $180^{\circ}$ & \\
\hline & & 17 & $180^{\circ}$ & \\
\hline & & 26 & $180^{\circ}$ & \\
\hline \multirow{8}{*}{$\mathrm{C}-2$} & & 3 & $180^{\circ}$ & \multirow{8}{*}{$\begin{array}{l}\text { Destroyed in } \\
\text { thermal-vac } \\
\text { test by burst } \\
\text { window }\end{array}$} \\
\hline & 1 & 4 & $0^{\circ}$ & \\
\hline & & 12 & $0^{\circ}$ & \\
\hline & & 18 & $0^{\circ}$ & \\
\hline & \multirow{4}{*}{2} & 6 & $180^{\circ}$ & \\
\hline & & 19 & $0^{\circ}$ & \\
\hline & & 10 & $0^{\circ}$ & \\
\hline & & 22 & $0^{\circ}$ & \\
\hline \multirow{8}{*}{ C-3 } & & 2 & $0^{\circ}$ & \multirow{8}{*}{$\begin{array}{l}\text { Flight } \\
\text { Collimator }\end{array}$} \\
\hline & 1 & 13 & $0^{\circ}$ & \\
\hline & & 23 & $0^{\circ}$ & \\
\hline & & 27 & $180^{\circ}$ & \\
\hline & \multirow{4}{*}{2} & 11 & $180^{\circ}$ & \\
\hline & & 16 & $180^{\circ}$ & \\
\hline & & 21 & $180^{\circ}$ & \\
\hline & & 25 & $180^{\circ}$ & \\
\hline
\end{tabular}

Table D.6 Collimator modules selected for the three assembled full collimators. The fourth column represents the orientation of each selected collimator module about its short $\mathrm{z}$-axis.

response functions were favored. Combinations of modules were selected based on constructing a full collimator response function with different degrees of flat top. This flat top arises from small misalignments between the collimator modules' axes. The width of the flat top for a full collimator is proportional to the $\sqrt{\text { variance }}$ of module response function centers.

The number of combinations for arranging the modules was increased by allowing a $\pi$-rotation about the module's short $\mathrm{z}$-axis. For such rotations the collimator $\mathrm{c}$-axis flips $\phi \rightarrow-\phi$ and rotates $\theta \rightarrow \theta+\pi$. Namely, $\theta$ effectively remains unchanged.

The response functions for the three full collimators constructed were simulated by combining the simulated response functions of each of its modules listed in Table D.6. The contour plots of the simulated response functions for the three full collimators are 


\begin{tabular}{|c|c|c|c|}
\hline Collimator & Response Fcn. & \multicolumn{2}{|c|}{ Flat Top } \\
\cline { 3 - 4 } & Maximum & $\Delta \phi$ & $\Delta \theta$ \\
\hline C-1 & 0.88 & $0.08^{\circ}$ & $0.14^{\circ}$ \\
C-2 & 0.90 & $0.08^{\circ}$ & $0.10^{\circ}$ \\
C-3 & 0.93 & $0.02^{\circ}$ & $0.04^{\circ}$ \\
\hline
\end{tabular}

Table D.7 Results of fitting the simulations of the response functions of collimators C-1, C-2 and C-3 for a flat top or plateau at the response function apex.

shown in Figure D.8. Their shapes follow the hexagonal geometry of the collimator tubes. As the response function gets closer to its apex, this hexagonal shape smears into an ellipse.

These simulations also show that collimators $\mathrm{C}-1$ and $\mathrm{C}-2$ have larger flat tops than collimator C-3. The width of these flat tops in $\phi$ and $\theta$, may be measured by fitting the simulated data of a $\phi$-scan and a $\theta$-scan. First, the simulated data along a scan is fit for a triangle. The difference between area under this triangle and the simulated data with a flat top is another triangle with area $A$. The width of the flat top is then simply given by

$$
\Delta_{\text {Flat Top }}=2 \sqrt{A \tan \theta_{\mathbf{t}}}
$$

where $\tan \theta_{\mathrm{t}}$ is defined by the fitted triangle, namely, base/2 divide by the height. Table D.7 shows the resulting widths and heights of the flat tops of collimators C-1, C-2 and C3. Collimator C-3 shows a flat top 3-4 times narrower than the other two collimators.

Collimators C-2 and C-3 were originally selected as the flight units for USA. While collimators C-1 and C-2 have very similar response functions, collimator C-3 was chosen over C-1 because its smaller flat top gave it the better angular resolution. Collimator C-2 was later replaced by C-1, after C-2 was damaged after a window break, as discussed in the next Section. Figures D.10 - D.25 show the measured scans of each module in collimators $\mathrm{C}-1$ and C-3 with their fitted response functions overplotted. 
Simulation of C-1 (installed in USA)

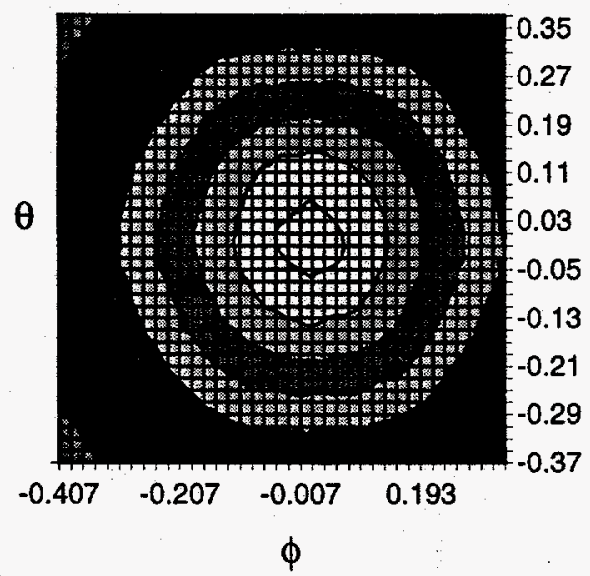

Simulation of C-2 (destroyed in thermal test)

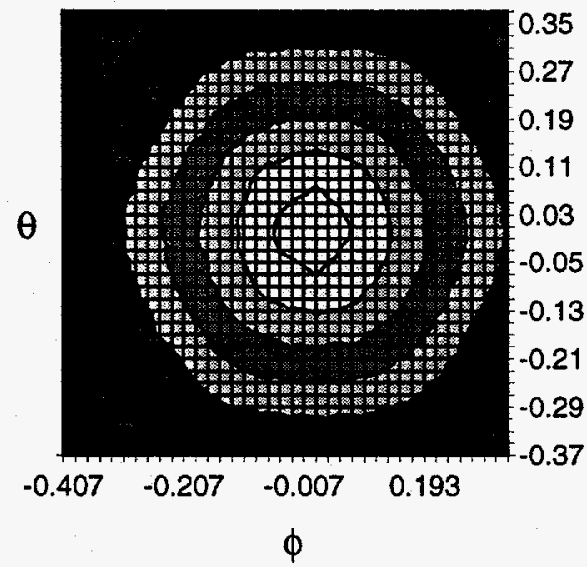

Simulation of C-3 (installed in USA)

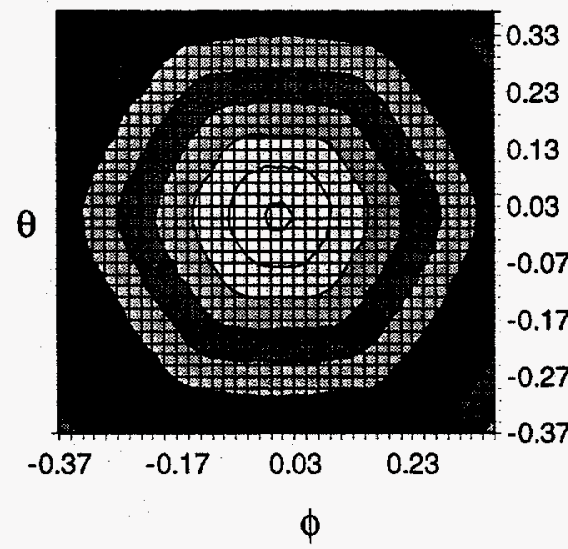

Fig. D.8 Simulated collimator response functions for collimators C-1, C-2 and C-3 from data taken in XRC test. 


\section{D.7 USA Collimator Failure}

In May of 1996, at the end of the last thermal-vac cycle of that series of tests, the window behind collimator C-2 burst, causing a catastrophic collimator failure. The window failure occurred between modules 10 and 22 and resulted in an large eye-shaped opening 2 1/4" wide at its center. As shown in Figure D.9, the bridge sheets between the two modules were left bowed into the copper tubes, collapsing a significant portion of the honeycomb structure.

The consensus after extensive discussion and analysis is that this collimator damage originated from a breach in the window that contained the proportional chamber gas volume. At the union of two collimator modules are two bridge sheets affixed together by a narrow bead of epoxy. The epoxy was intended to prevent relative movements between the modules, not to withstand substantial loads. The loads that were expected were along the collimator face due to the gas pressure.

This union formed a slit and the two bridge sheets that defined its aperture had semi-sharp edges. The gas is believed to have pushed the window into these slits, where the sharp edges eventually breached the integrity of the window. As the gas then leaked into the space between the modules, the pressure between the two bridge sheets rose rapidly until the epoxy failed and a runaway of pressure from the remaining gas volume collapsed the surrounding honeycomb. An engineering calculation was able to reproduce the general character of this model quantitatively.

To prevent this type of failure from reoccurring, all of the slits between adjacent collimator modules were subsequently taped over for remaining USA collimators $\mathrm{C}-1$ and C-3. The tape helps prevent the window from being cut by the semi-sharp edges of the bridge sheets and from letting the window push into the slits. The thermal-vac tests were then repeated on the USA instrument containing collimators $\mathrm{C}-1$ and $\mathrm{C}-3$ and no additional collimator failures occurred. 

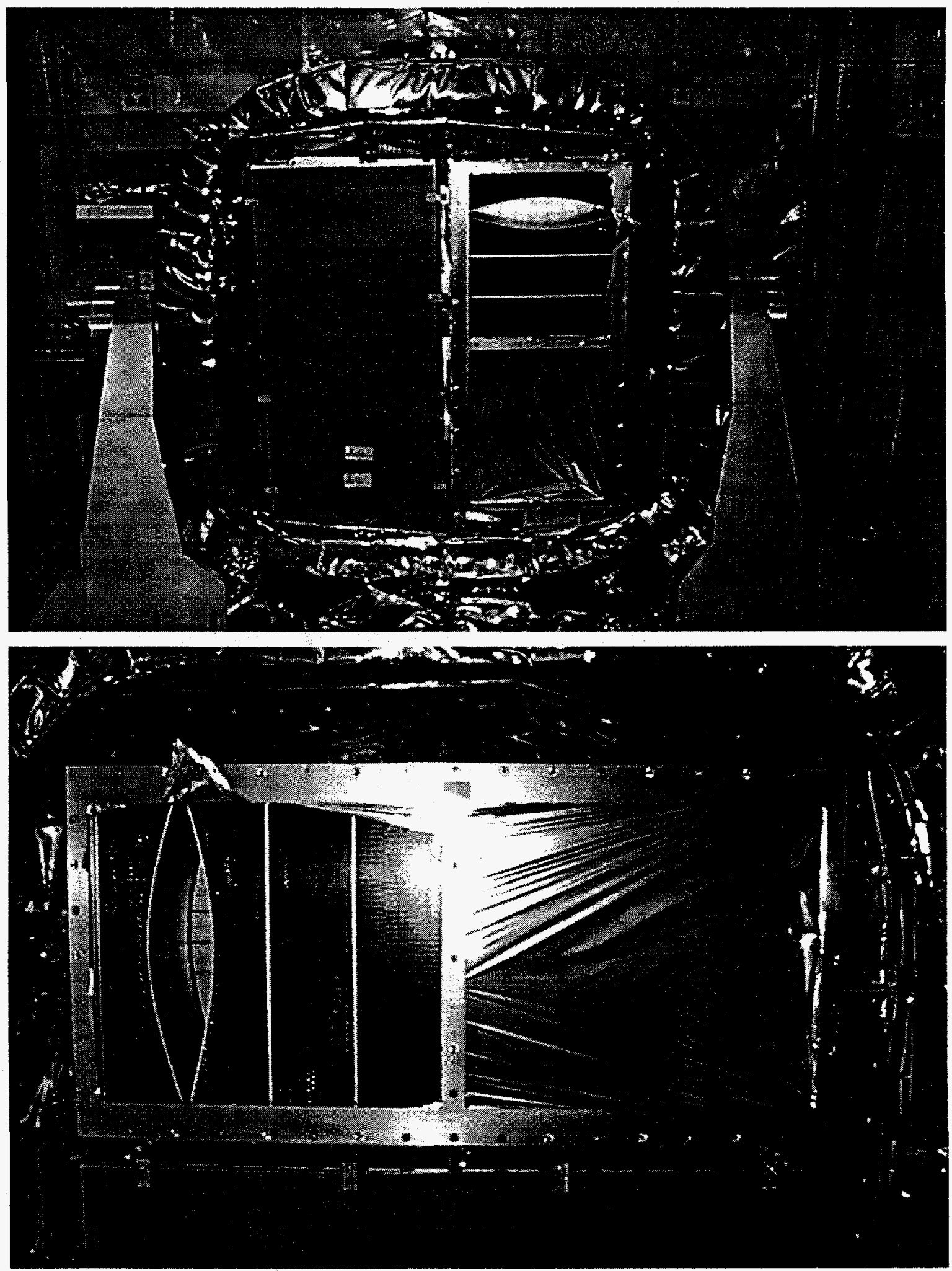

Fig. D.9 Damage from burst PWC window behind USA collimator C-2 resulting in a large eye-shaped opening. The top image shows the hole relative to the USA instrument, and the bottom image shows a close-up of collimator C- 2 . 

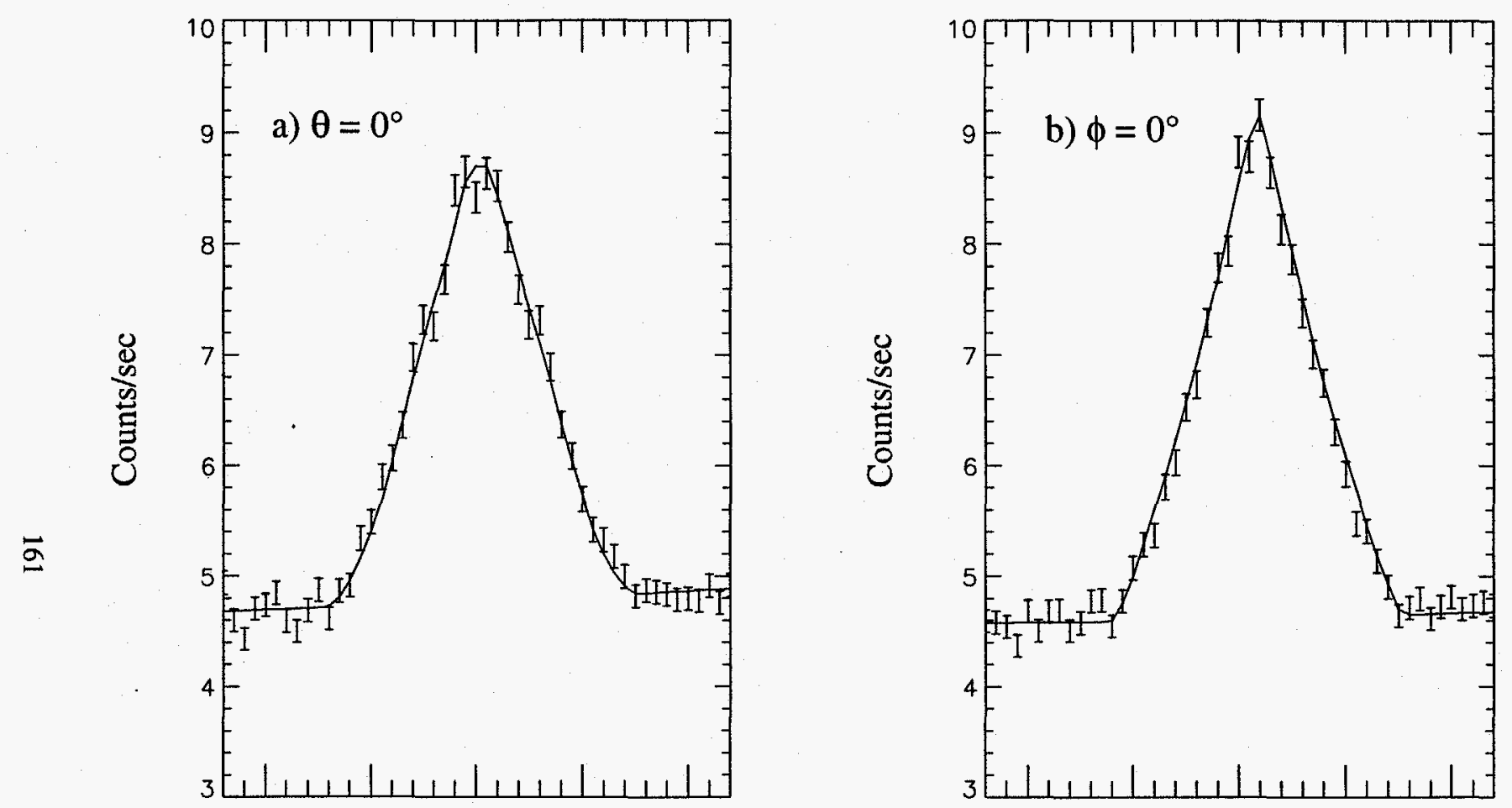

Fig. D.10 :

Scans in a) $\phi$ (yaw) and in b) $\theta$ (pitch) for unit:

Collimator: C-1

$\begin{array}{ll}\text { Segment: } & 1 \\ \text { Module: } & 5\end{array}$

Simulated response

function using parameters

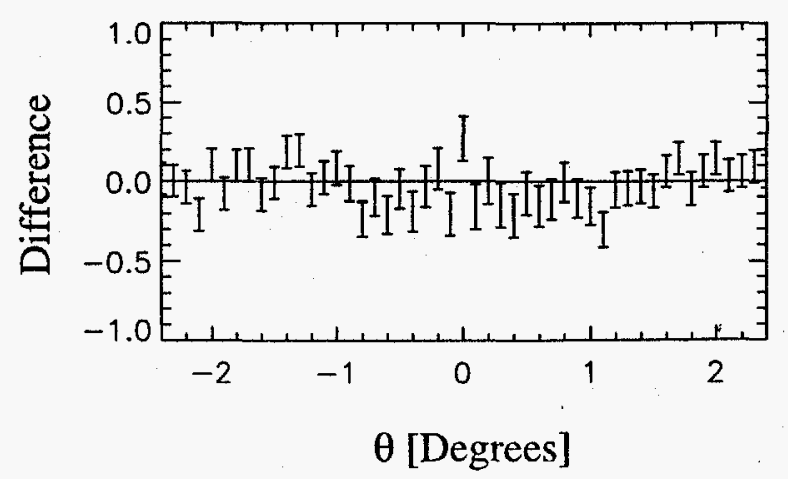

$(\phi),(\theta)$

FWHM: $1.24^{\circ}, 1.16^{\circ}$

Centers: $0.037^{\circ}, 0.175^{\circ}$

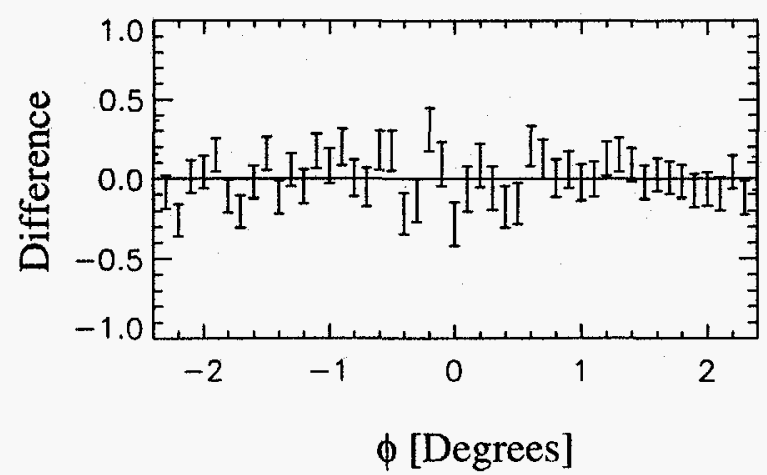

from fit to data are shown in solid curve. Difference between data and simulation shown in lower curves. 

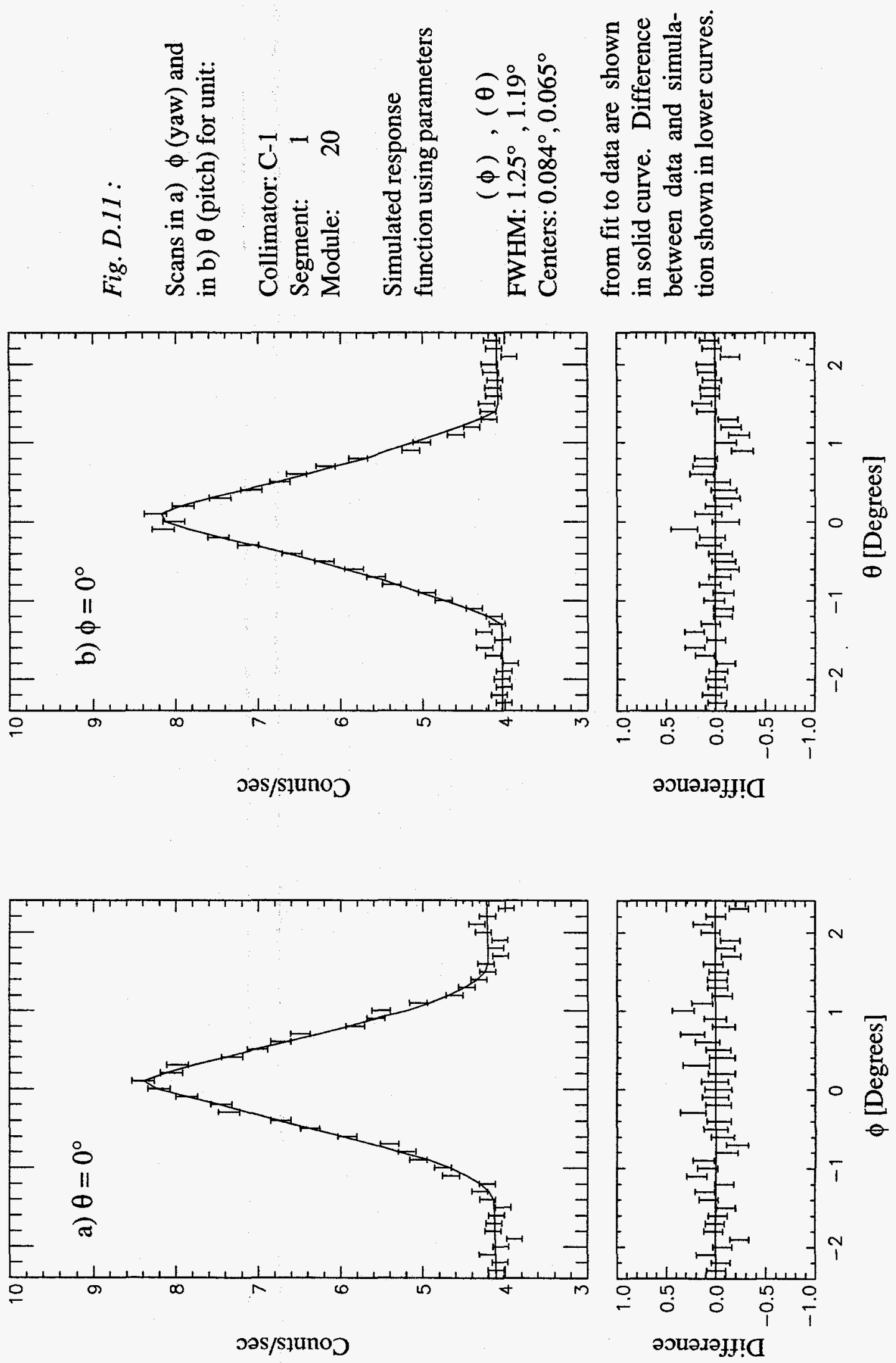

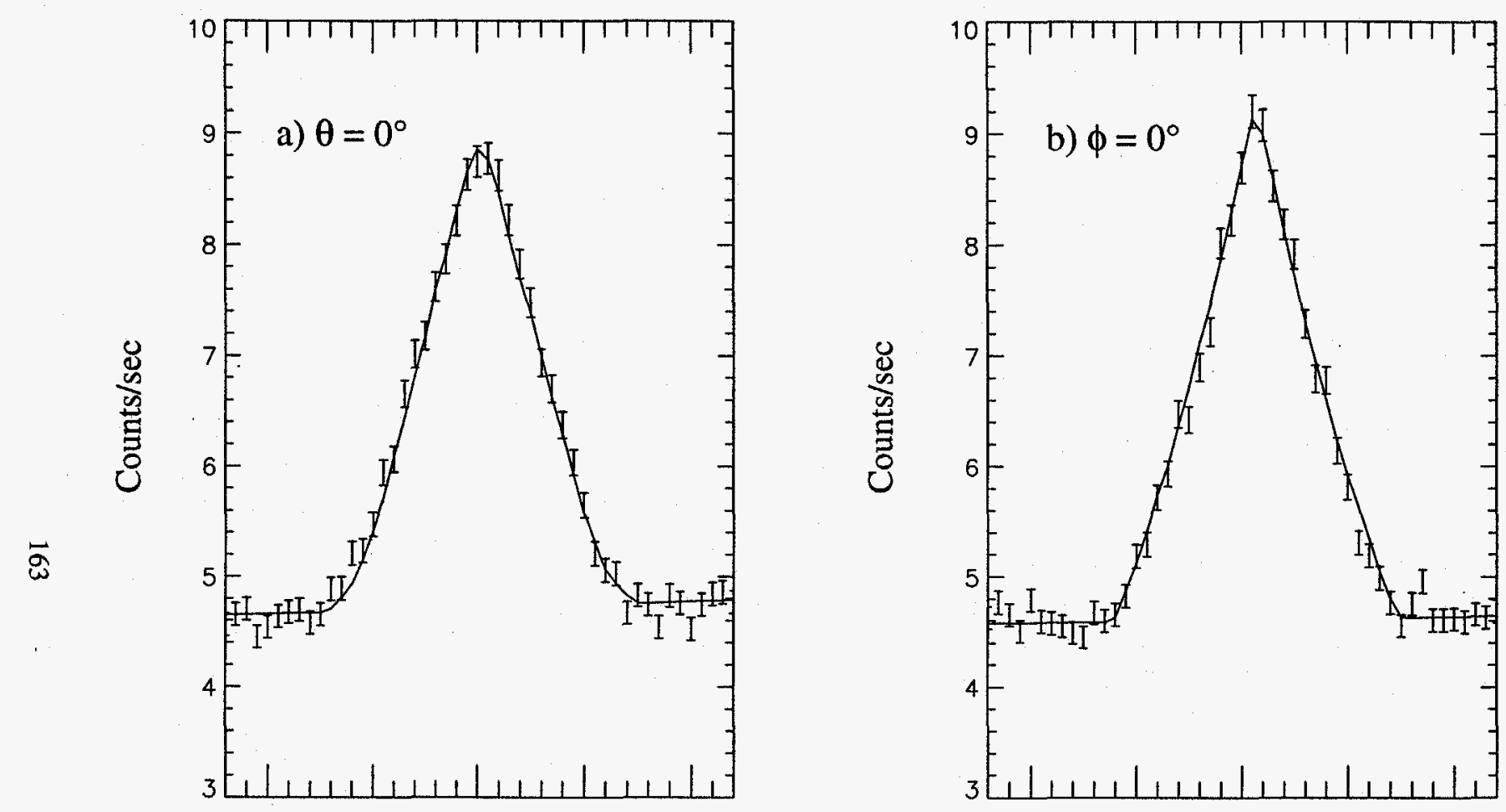

Fig. D.12 :

Scans in a) $\phi$ (yaw) and in b) $\theta$ (pitch) for unit:

\section{Collimator: C-1}

$\begin{array}{ll}\text { Segment: } & 1 \\ \text { Module: } & 8\end{array}$

Simulated response

function using parameters

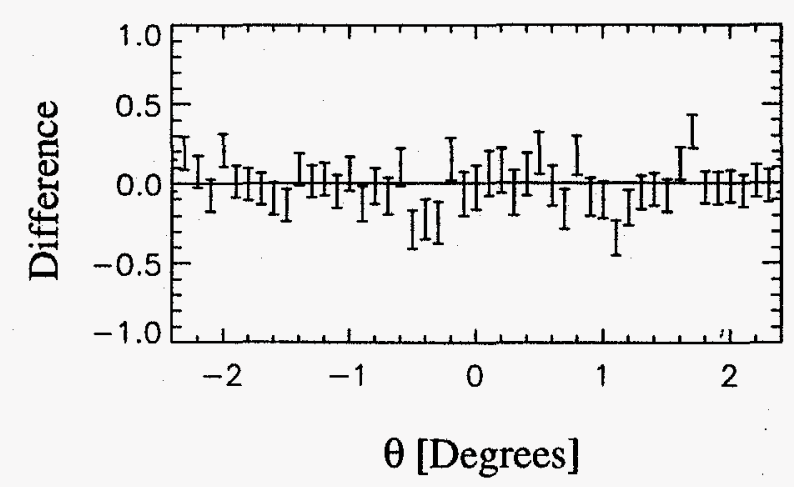

( $\phi),(\theta)$

FWHM: $1.25^{\circ}, 1.20^{\circ}$

Centers: $0.021^{\circ}, 0.130^{\circ}$

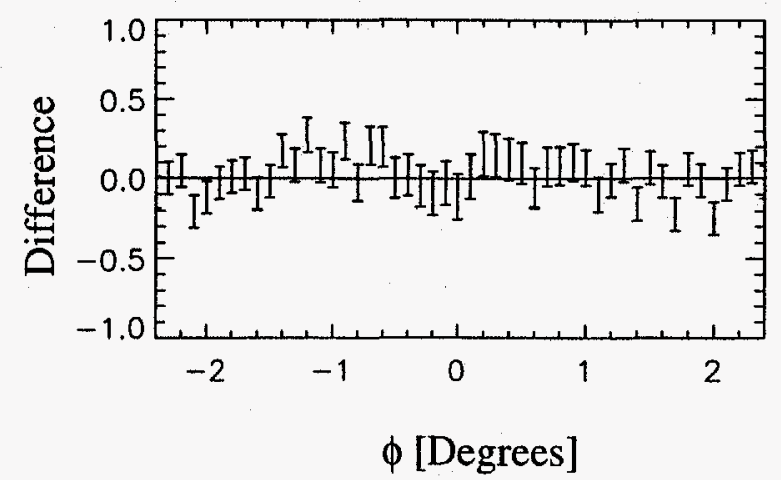

from fit to data are shown in solid curve. Difference between data and simulation shown in lower curves. 

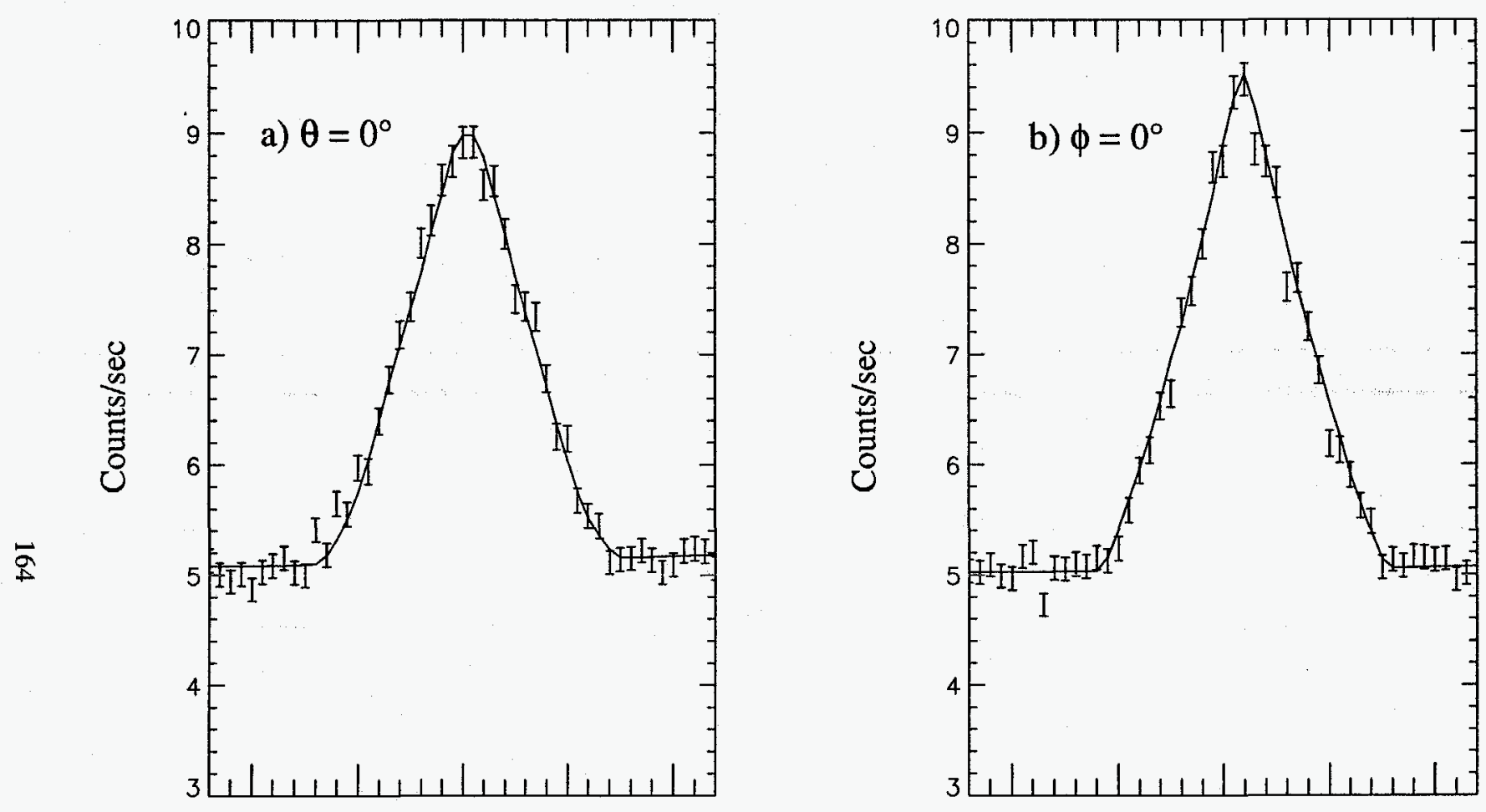

Fig. D.13 :

Scans in a) $\phi$ (yaw) and in b) $\theta$ (pitch) for unit:

Collimator: C-1

Segment: $\quad 1$

Module: 14

Simulated response

function using parameters

$$
(\phi),(\theta)
$$

FWHM: $1.28^{\circ}, 1.17^{\circ}$

Centers: $0.043^{\circ}, 0.191^{\circ}$
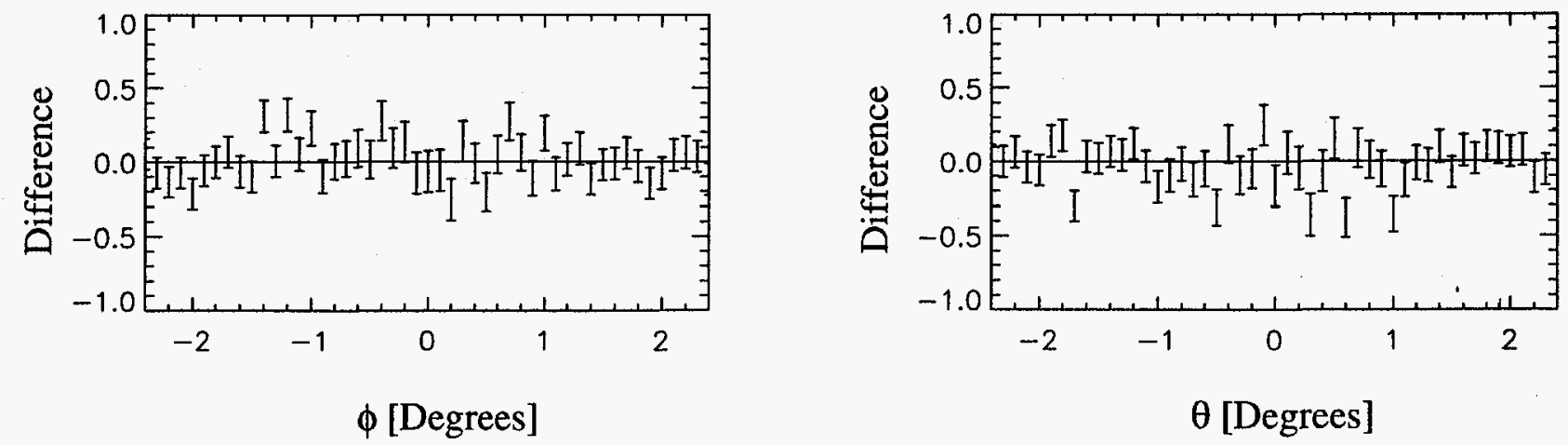

from fit to data are shown in solid curve. Difference between data and simulation shown in lower curves. 

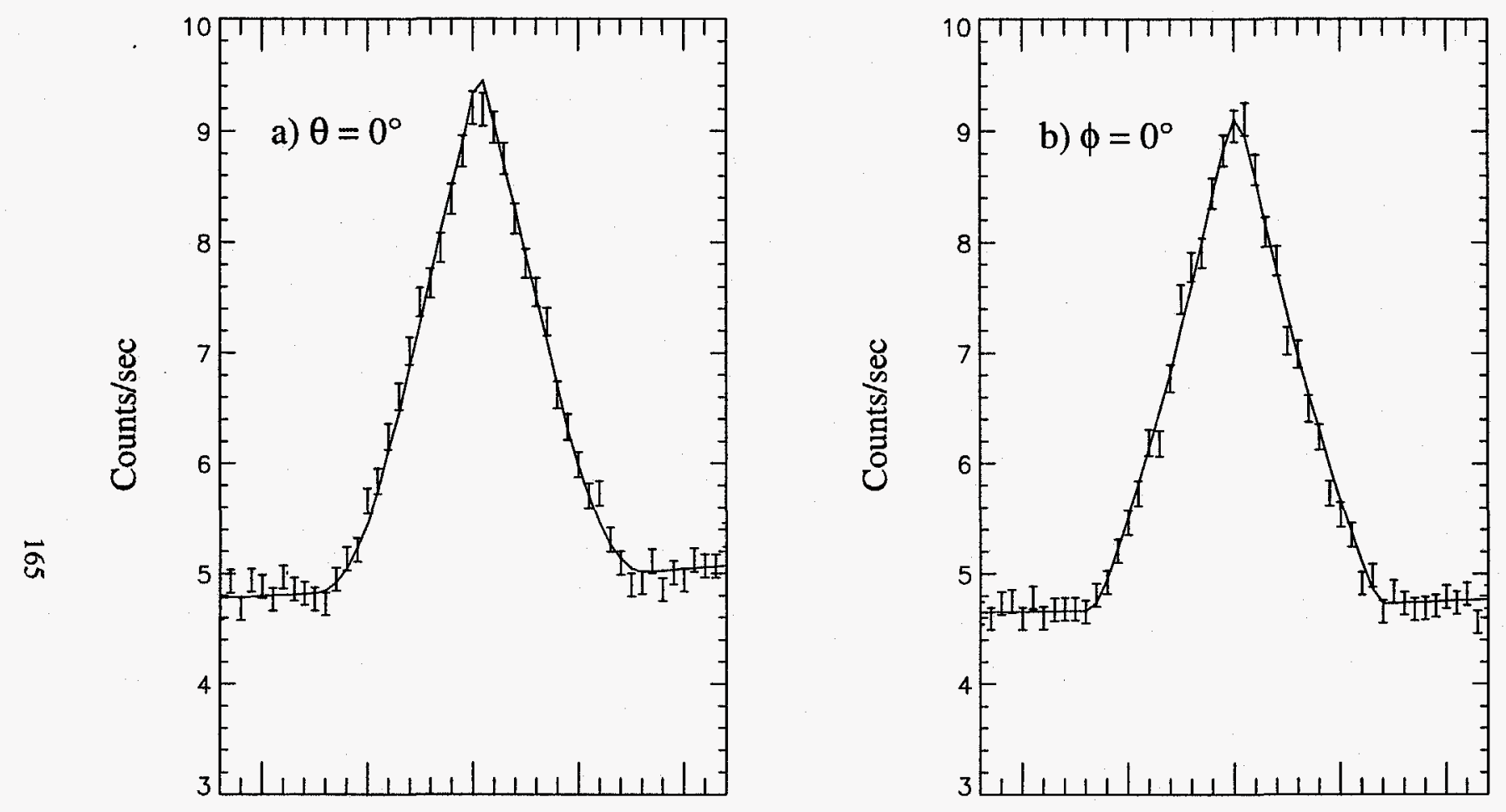

Fig. D.14 :

Scans in a) $\phi$ (yaw) and in b) $\theta$ (pitch) for unit:

\section{Collimator: C-1}

Segment: 2

Module: 1

Simulated response

function using parameters

$$
(\phi),(\theta)
$$

FWHM: $1.25^{\circ}, 1.17^{\circ}$

Centers: $0.070^{\circ}, 0.016^{\circ}$
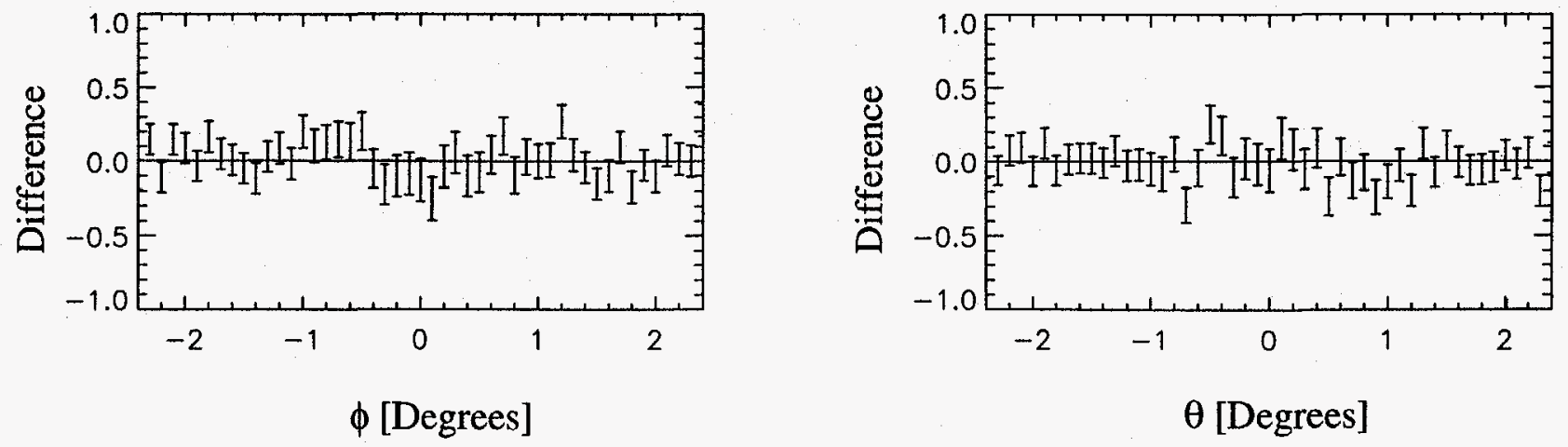

from fit to data are shown in solid curve. Difference between data and simulation shown in lower curves. 

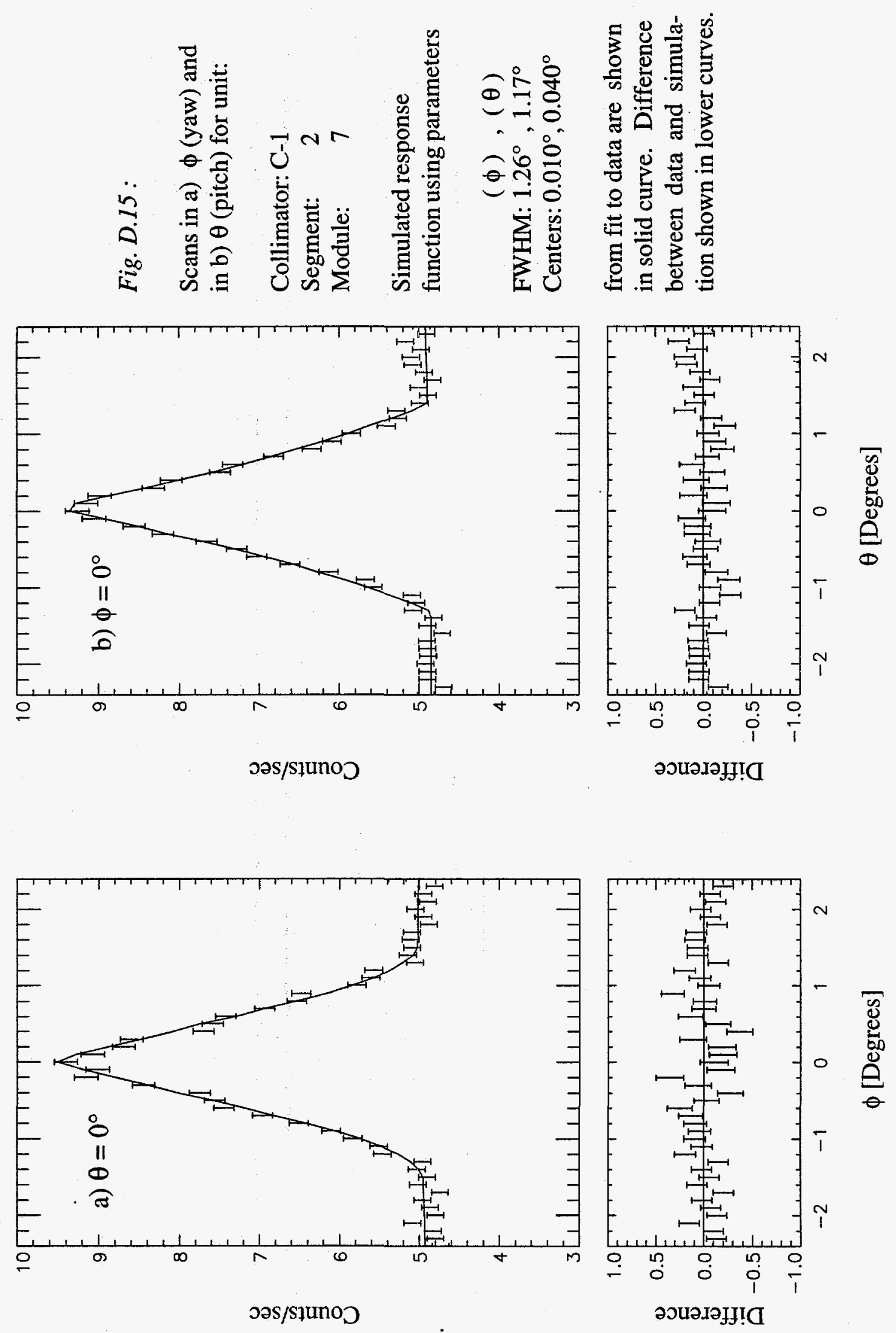

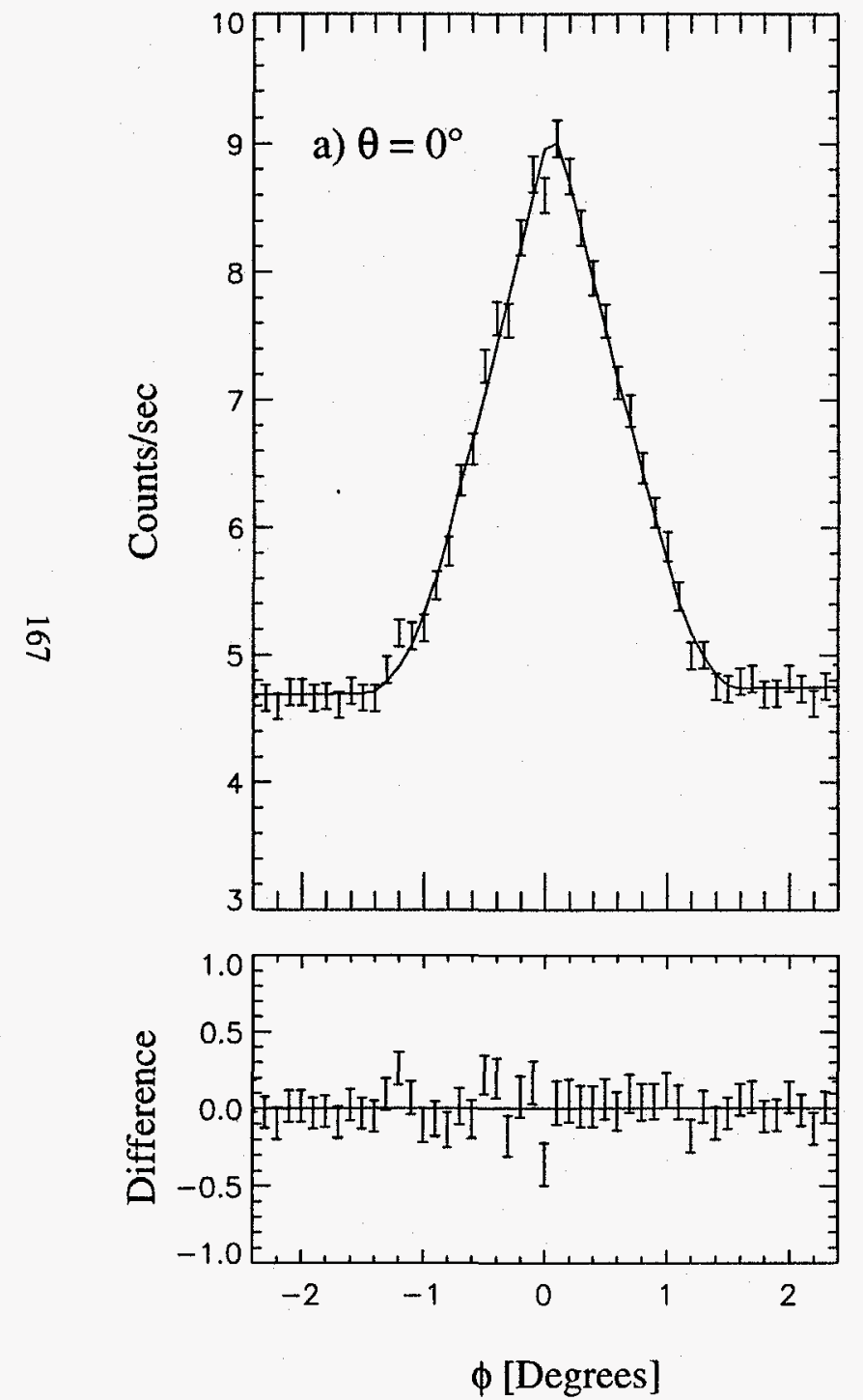
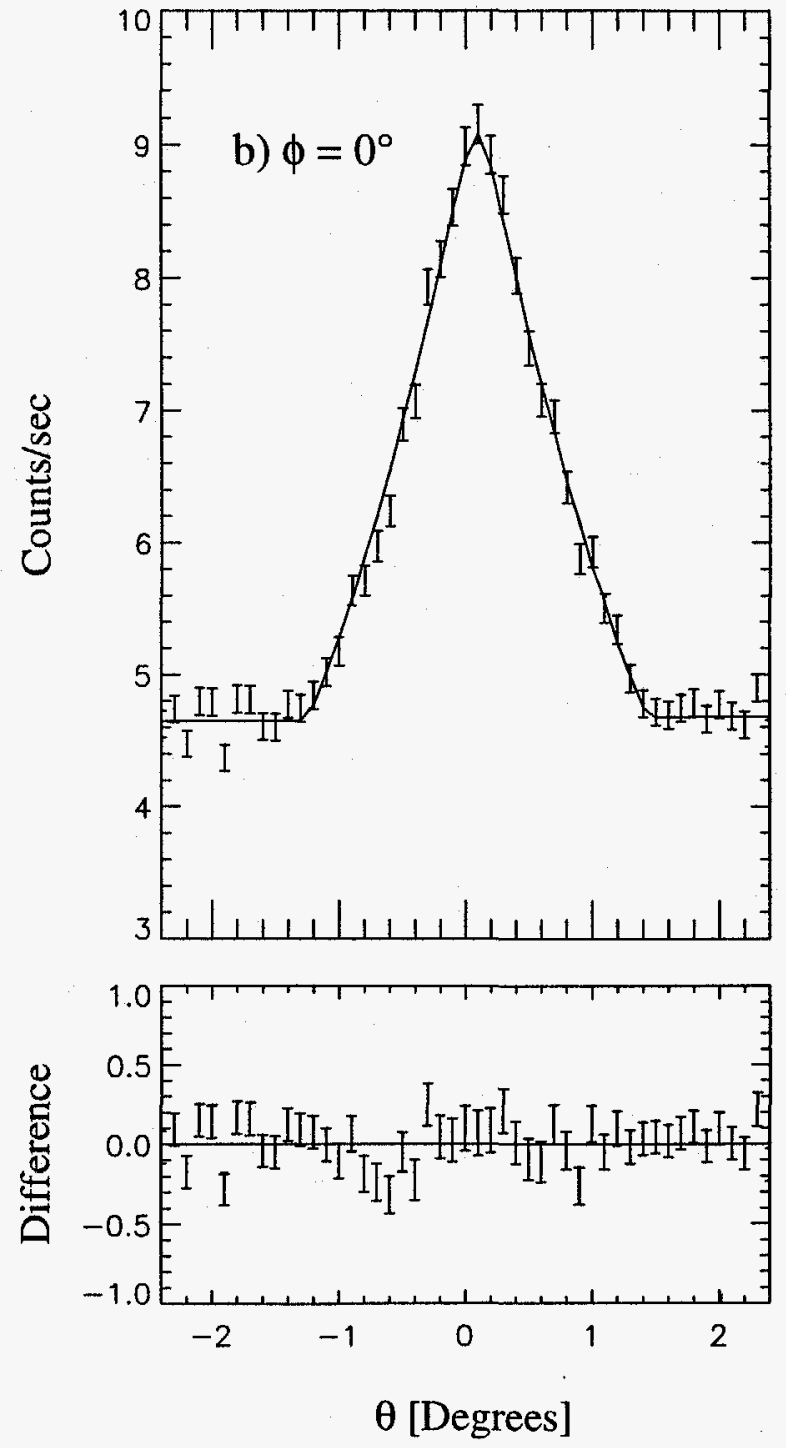

Fig. D.16:

Scans in a) $\phi$ (yaw) and in b) $\theta$ (pitch) for unit:

Collimator: C-1

Segment: 2

Module: 17

Simulated response function using parameters

( $\phi),(\theta)$

FWHM: $1.22^{\circ}, 1.18^{\circ}$

Centers: $0.063^{\circ}, 0.089^{\circ}$

from fit to data are shown in solid curve. Difference between data and simulation shown in lower curves. 

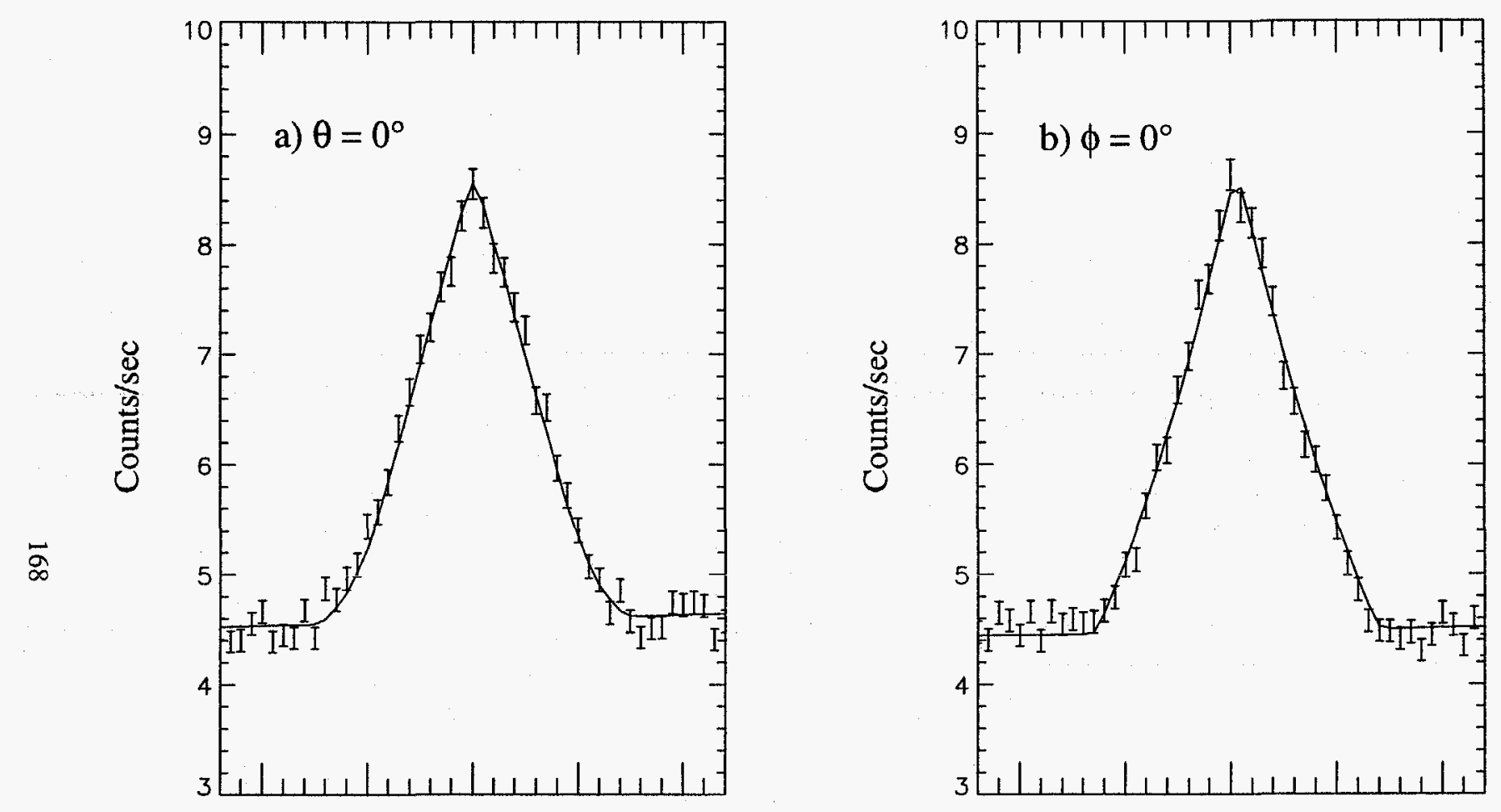

Fig. D.17 :

Scans in a) $\phi$ (yaw) and in b) $\theta$ (pitch) for unit:

Collimator: $\mathrm{C}-1$

Segment: 2

Module: $\quad 26$

Simulated response

function using parameters

$$
(\phi),(\theta)
$$

FWHM: $1.27^{\circ}, 1.19^{\circ}$

Centers: $0.008^{\circ}, 0.058^{\circ}$
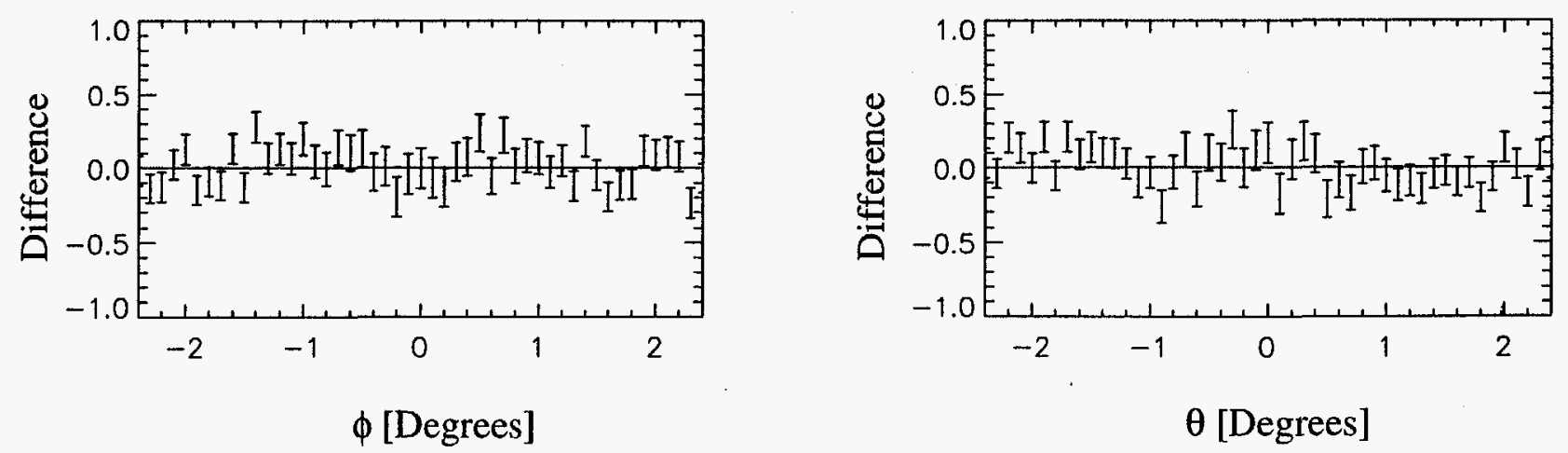

from fit to data are shown in solid curve. Difference between data and simulation shown in lower curves. 

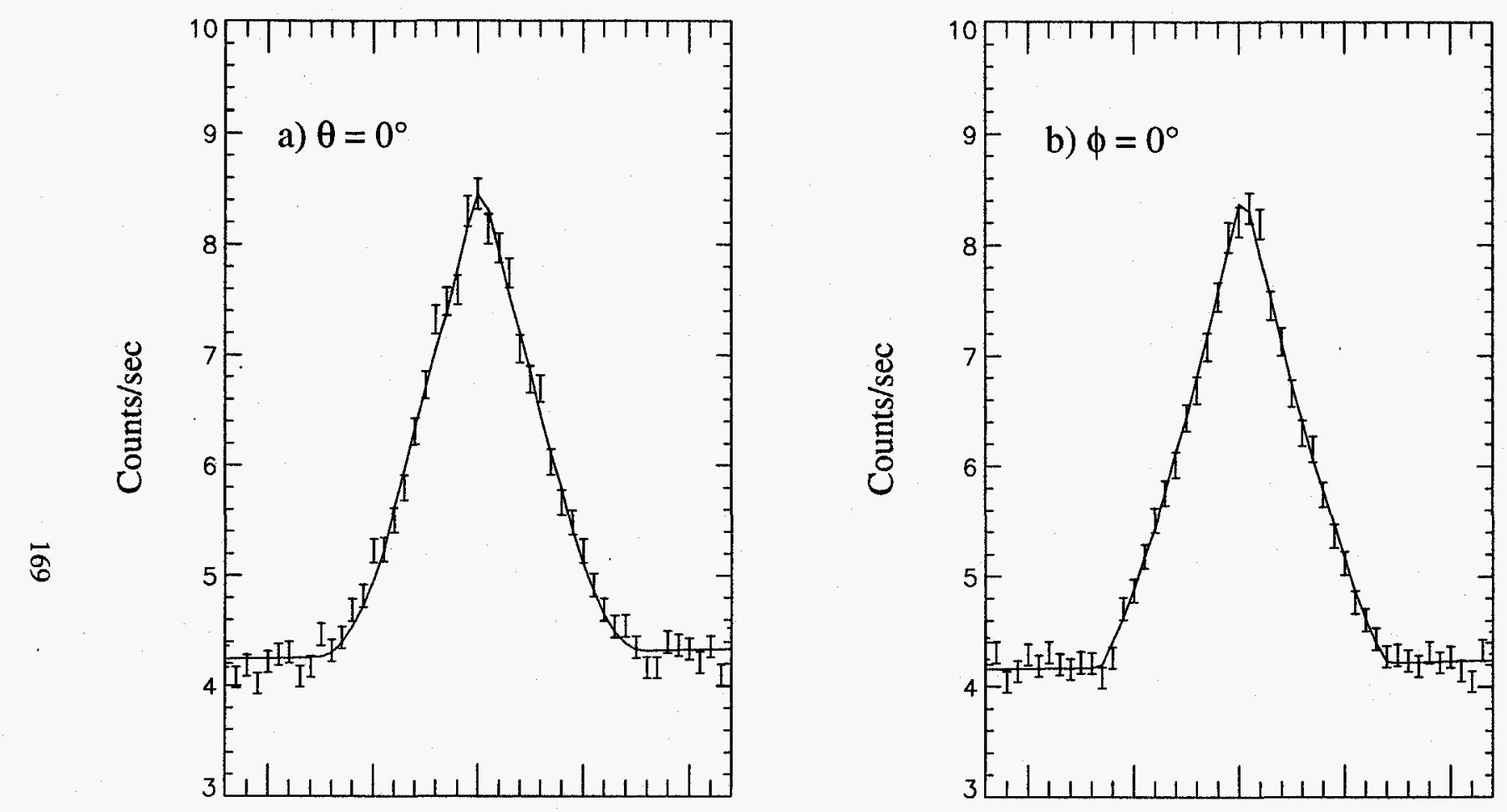

Fig. D.18:

Scans in a) $\phi$ (yaw) and in b) $\theta$ (pitch) for unit:

Collimator: C-3

Segment: $\quad 1$

Simulated response

function using parameters

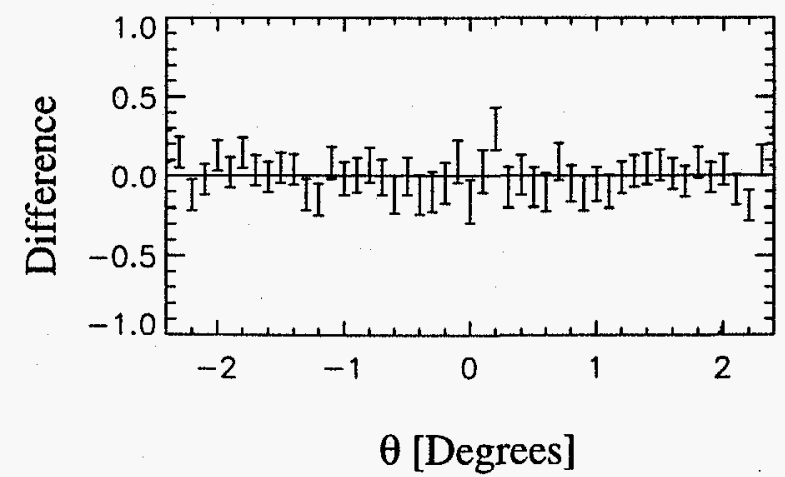

( $\phi),(\theta)$

FWHM: $1.24^{\circ}, 1.20^{\circ}$

Centers: $0.019^{\circ}, 0.042^{\circ}$

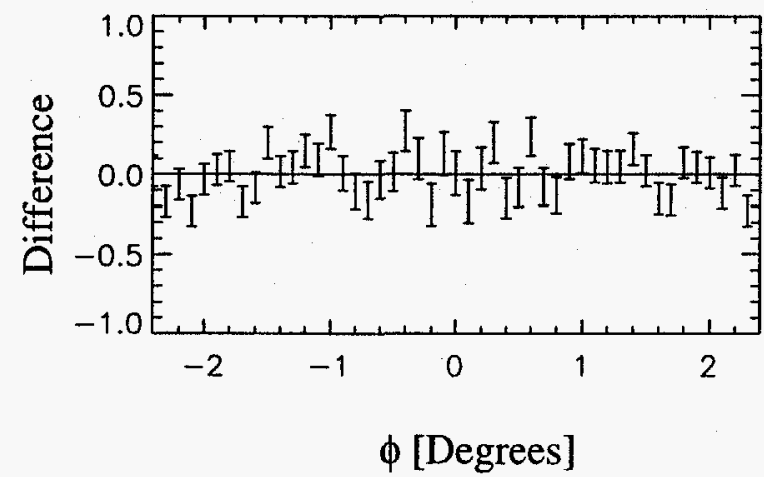

from fit to data are shown in solid curve. Difference between data and simulation shown in lower curves. 

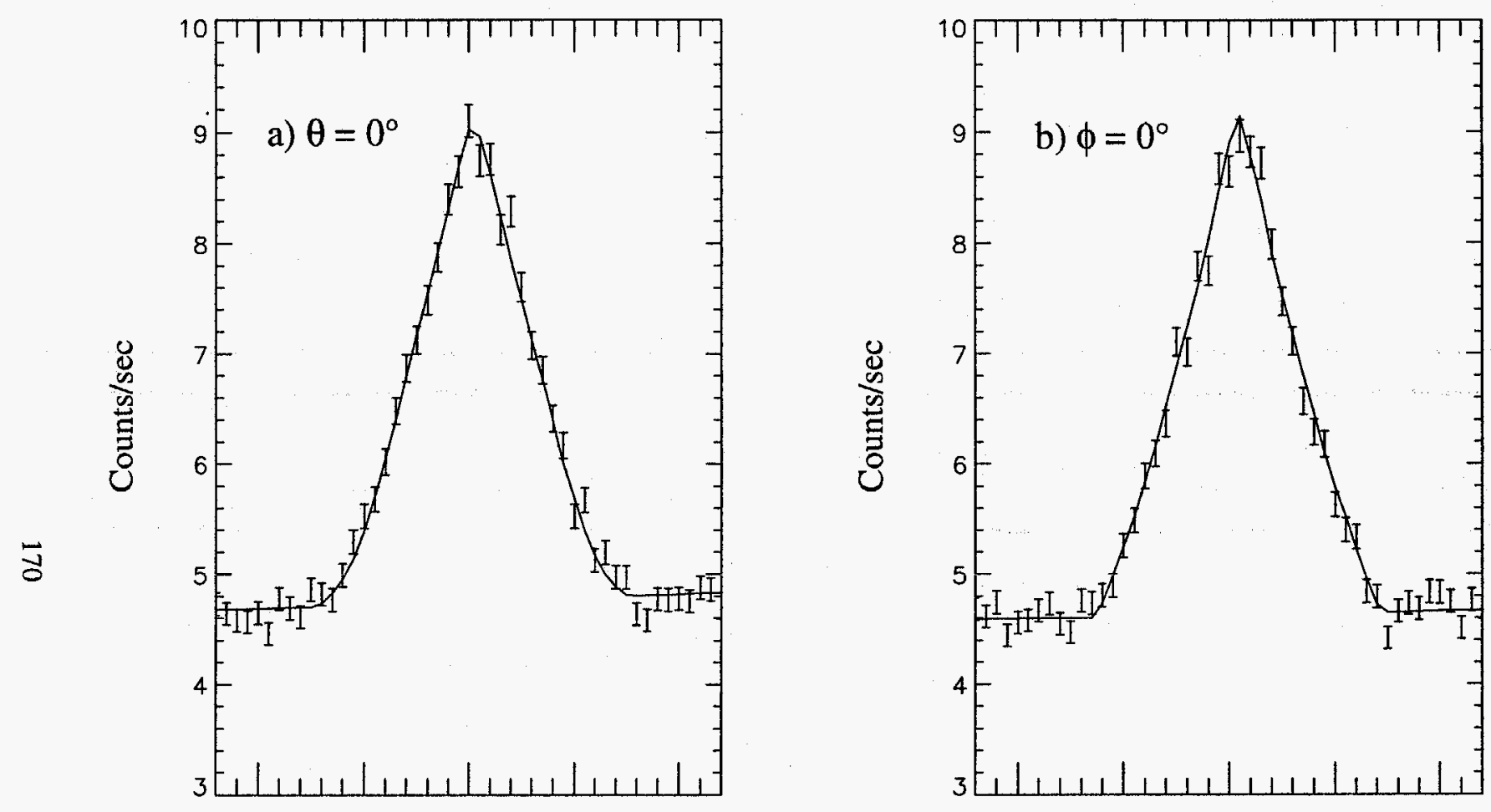

Fig. D.19:

Scans in a) $\phi$ (yaw) and in b) $\theta$ (pitch) for unit:

Collimator: C-3

Segment: 1

Module: 13

Simulated response

function using parameters

$$
(\phi),(\theta)
$$

FWHM: $1.23^{\circ}, 1.20^{\circ}$

Centers: $0.037^{\circ}, 0.088^{\circ}$
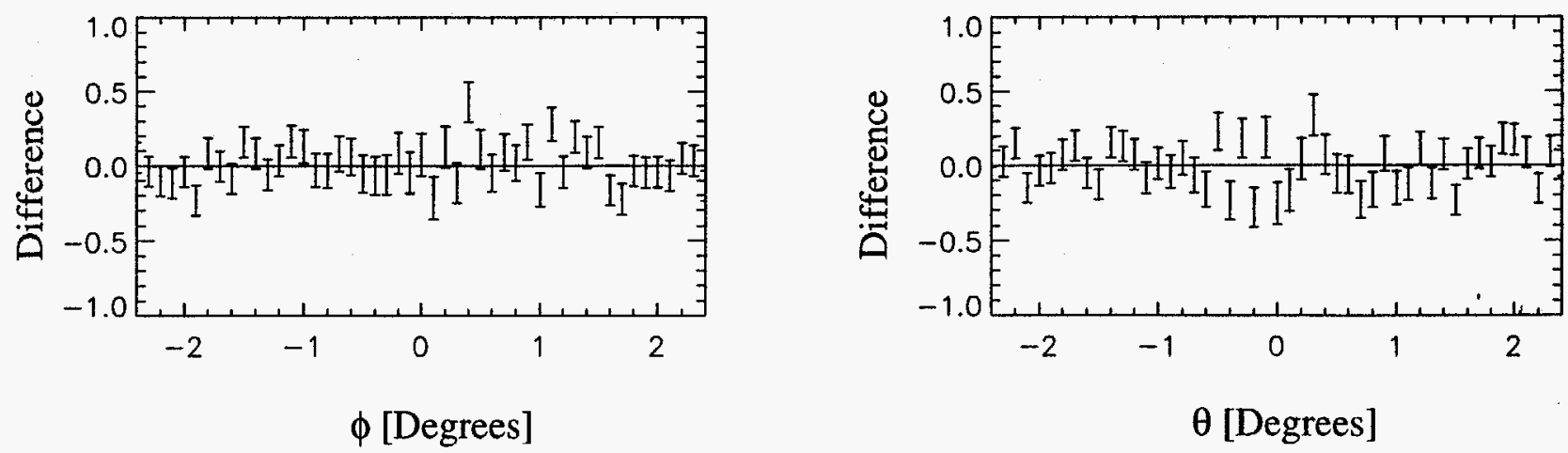

from fit to data are shown in solid curve. Difference between data and simulation shown in lower curves. 

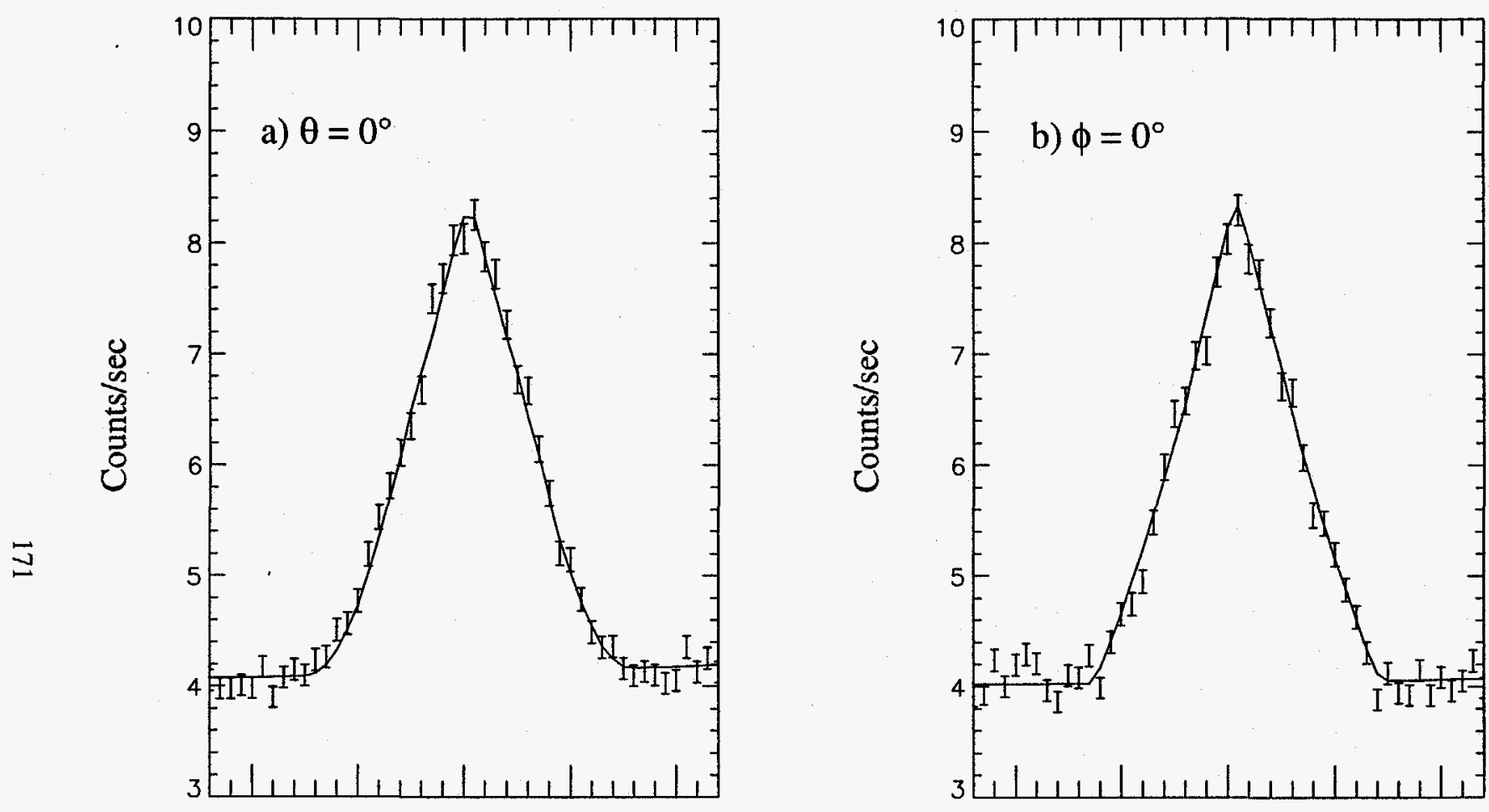

Fig. D.20 :

Scans in a) $\phi$ (yaw) and in b) $\theta$ (pitch) for unit:

Collimator: C-3

Segment: 1

Module: 23

Simulated response function using parameters

$$
(\phi),(\theta)
$$

FWHM: $1.27^{\circ}, 1.21^{\circ}$

Centers: $0.045^{\circ}, 0.085^{\circ}$
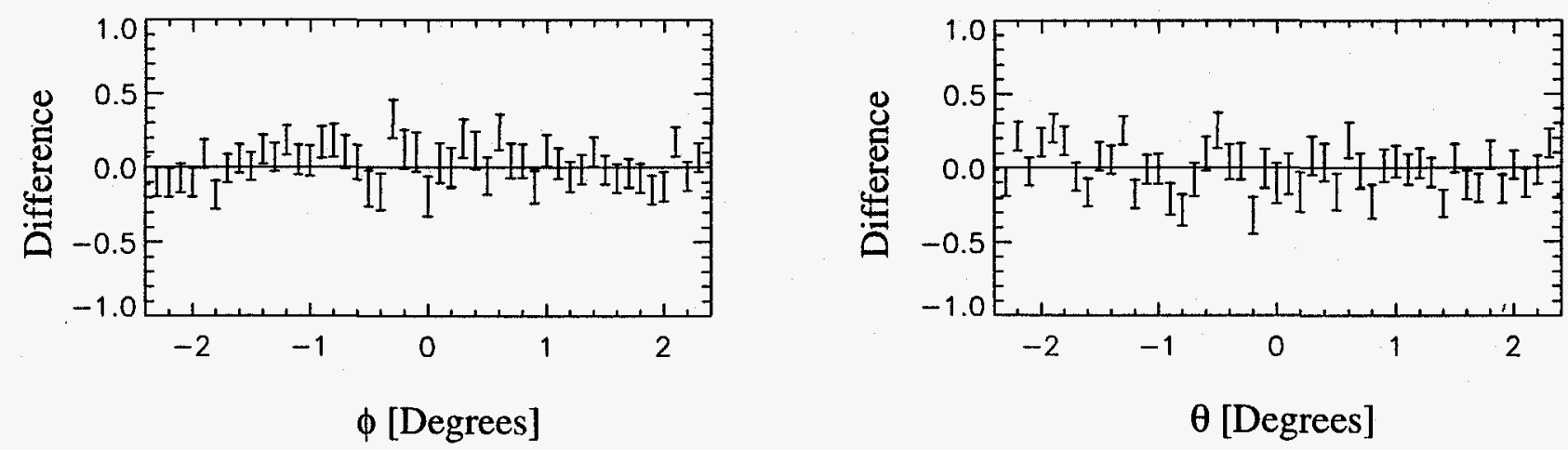

from fit to data are shown in solid curve. Difference between data and simulation shown in lower curves. 

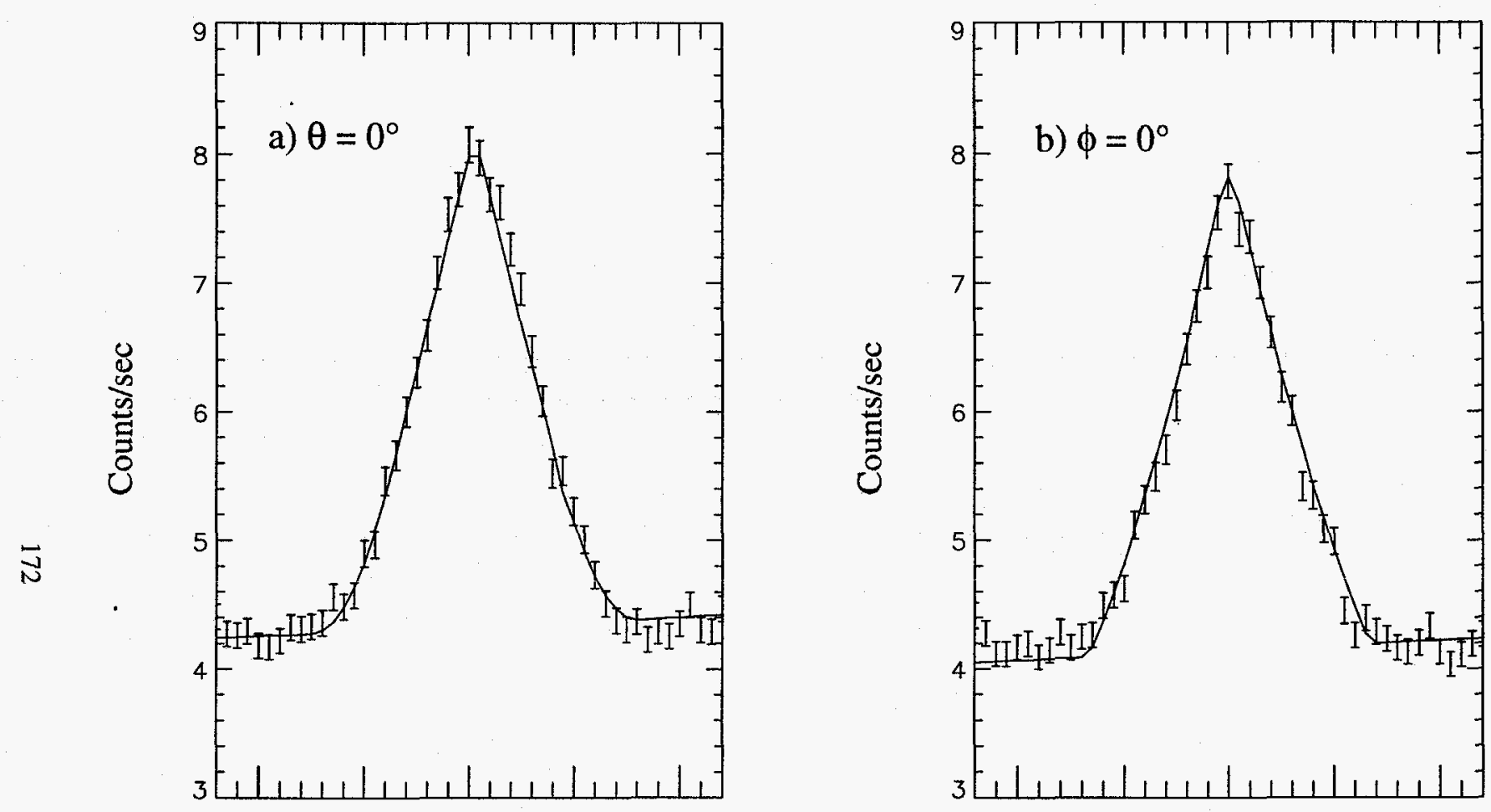

Fig. D.21 :

Scans in a) $\phi$ (yaw) and in b) $\theta$ (pitch) for unit:

\section{Collimator: C-3}

Segment: 1

Module: $\quad 27$

Simulated response

function using parameters

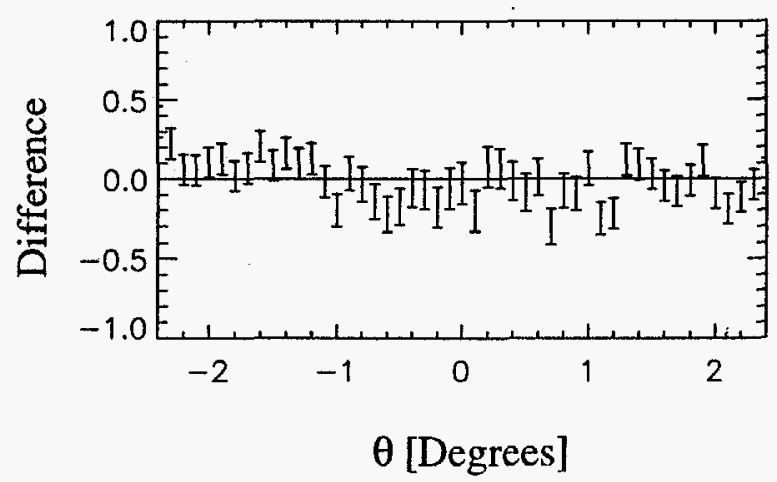

, $(\theta)$

FWHM: $1.23^{\circ}, 1.17^{\circ}$

Centers: $0.052^{\circ}, 0.006^{\circ}$

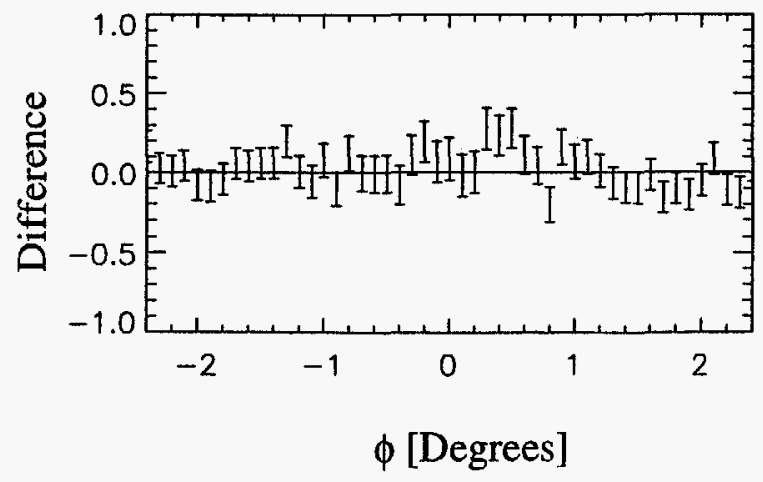

from fit to data are shown in solid curve. Difference between data and simulation shown in lower curves. 

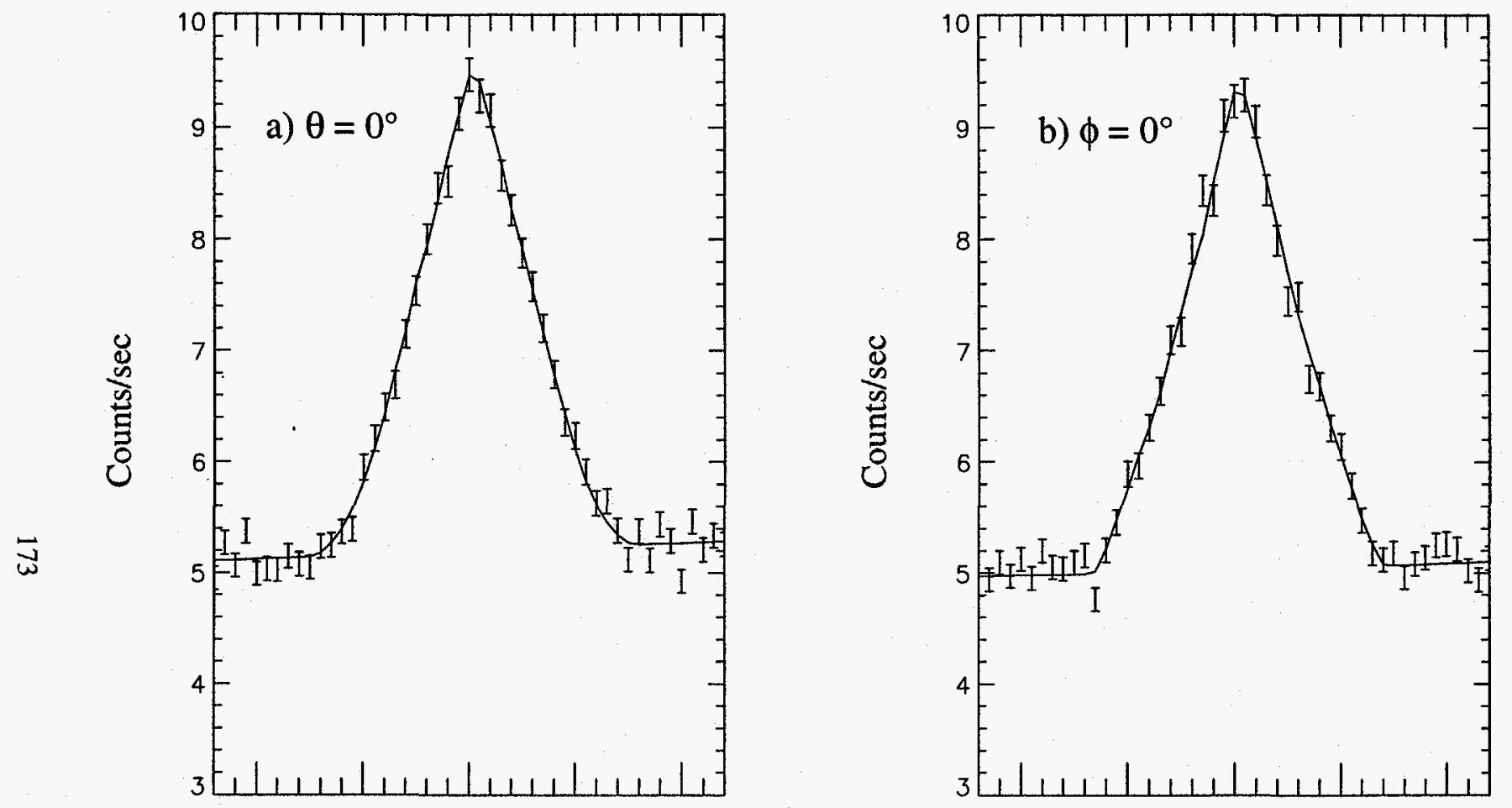

Fig. D.22 :

Scans in a) $\phi$ (yaw) and in b) $\theta$ (pitch) for unit:

Collimator: C-3

Segment: 2

Module: 11

Simulated response

function using parameters

$(\phi),(\theta)$

FWHM: $1.20^{\circ}, 1.20^{\circ}$

Centers: $0.038^{\circ}, 0.046^{\circ}$
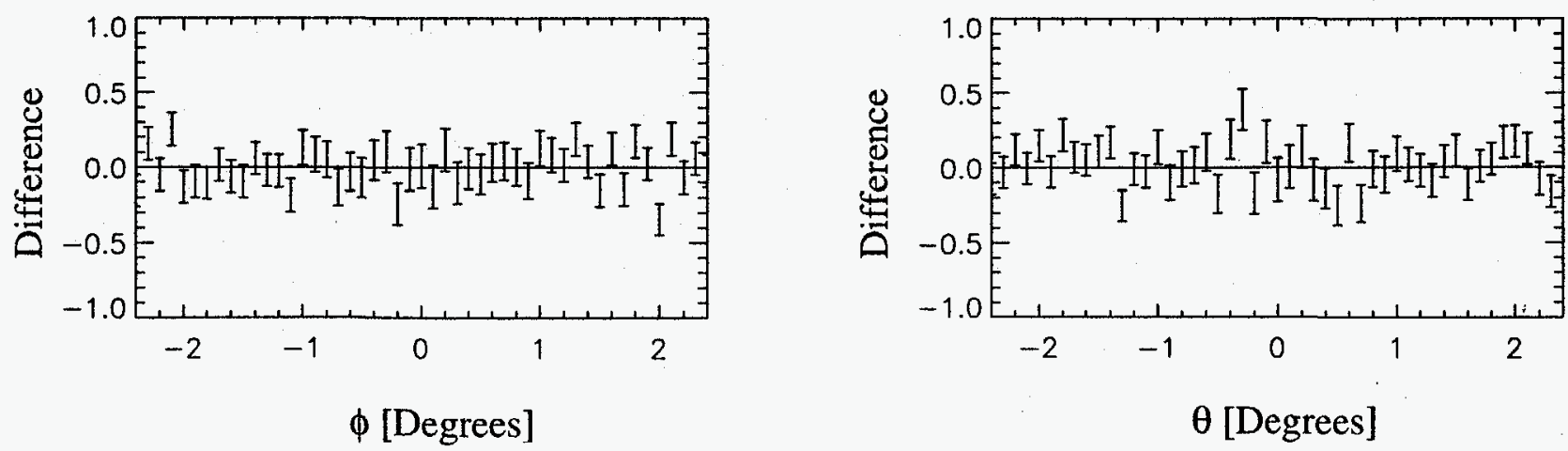

from fit to data are shown in solid curve. Difference between data and simulation shown in lower curves. 

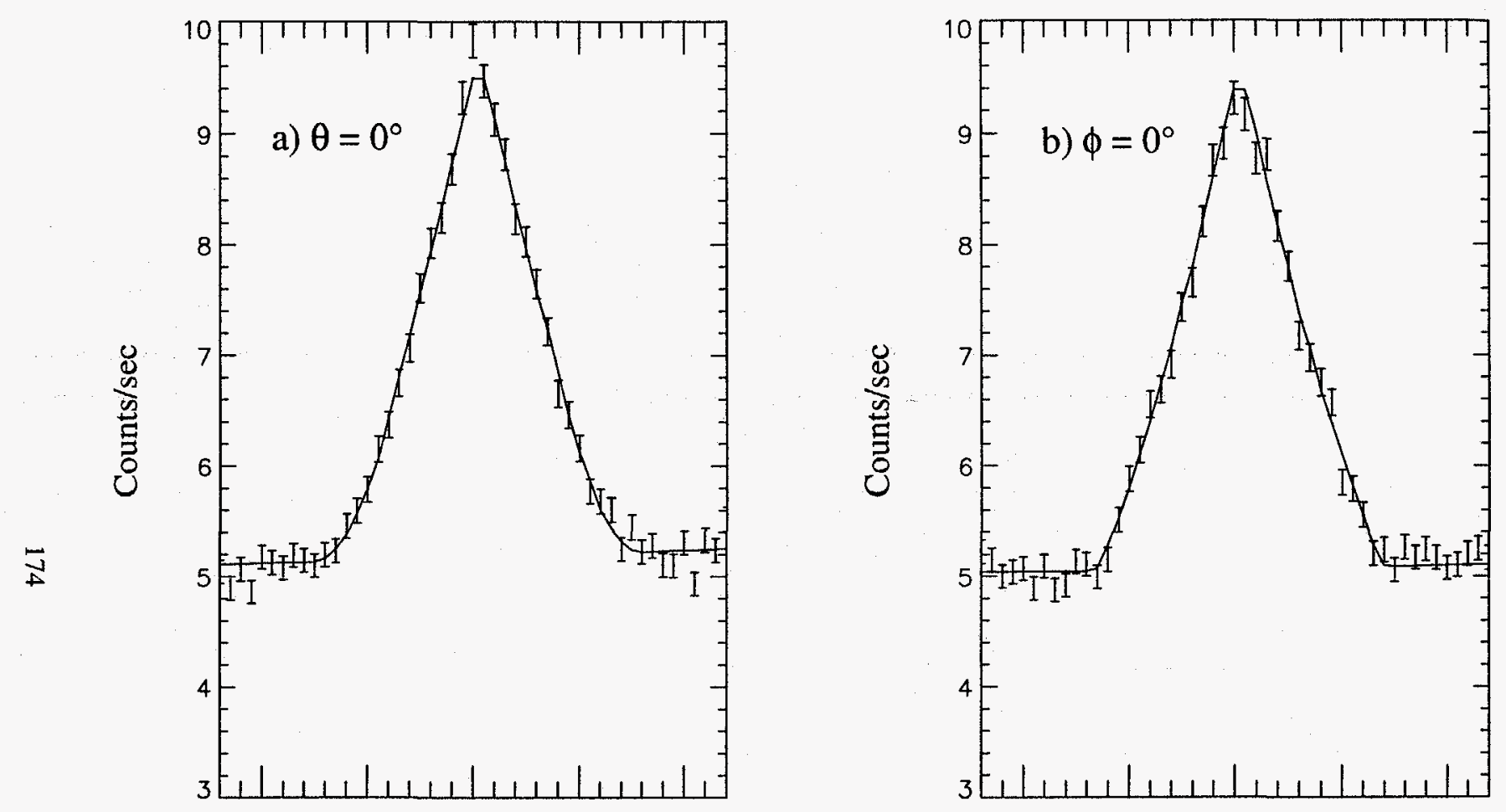

Fig. D.23 :

Scans in a) $\phi$ (yaw) and in b) $\theta$ (pitch) for unit:

Collimator: C-3

Segment: 2

Module: 16

Simulated response

function using parameters

$$
\text { ( } \phi),(\theta)
$$

FWHM: $1.21^{\circ}, 1.20^{\circ}$

Centers: $0.048^{\circ}, 0.044^{\circ}$
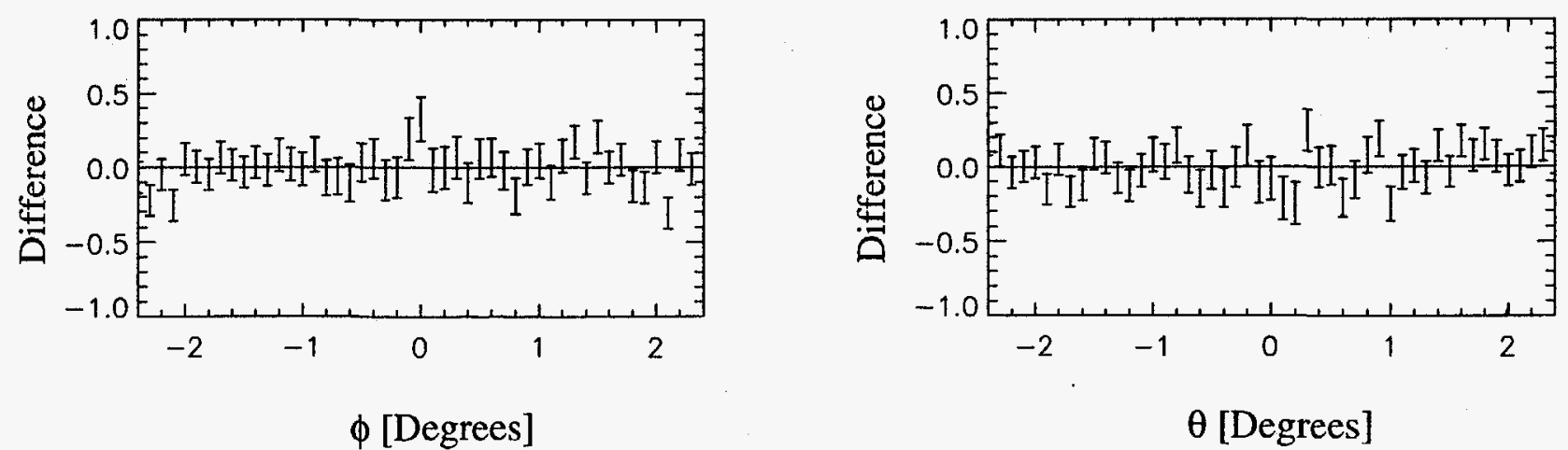

from fit to data are shown in solid curve. Difference between data and simulation shown in lower curves. 

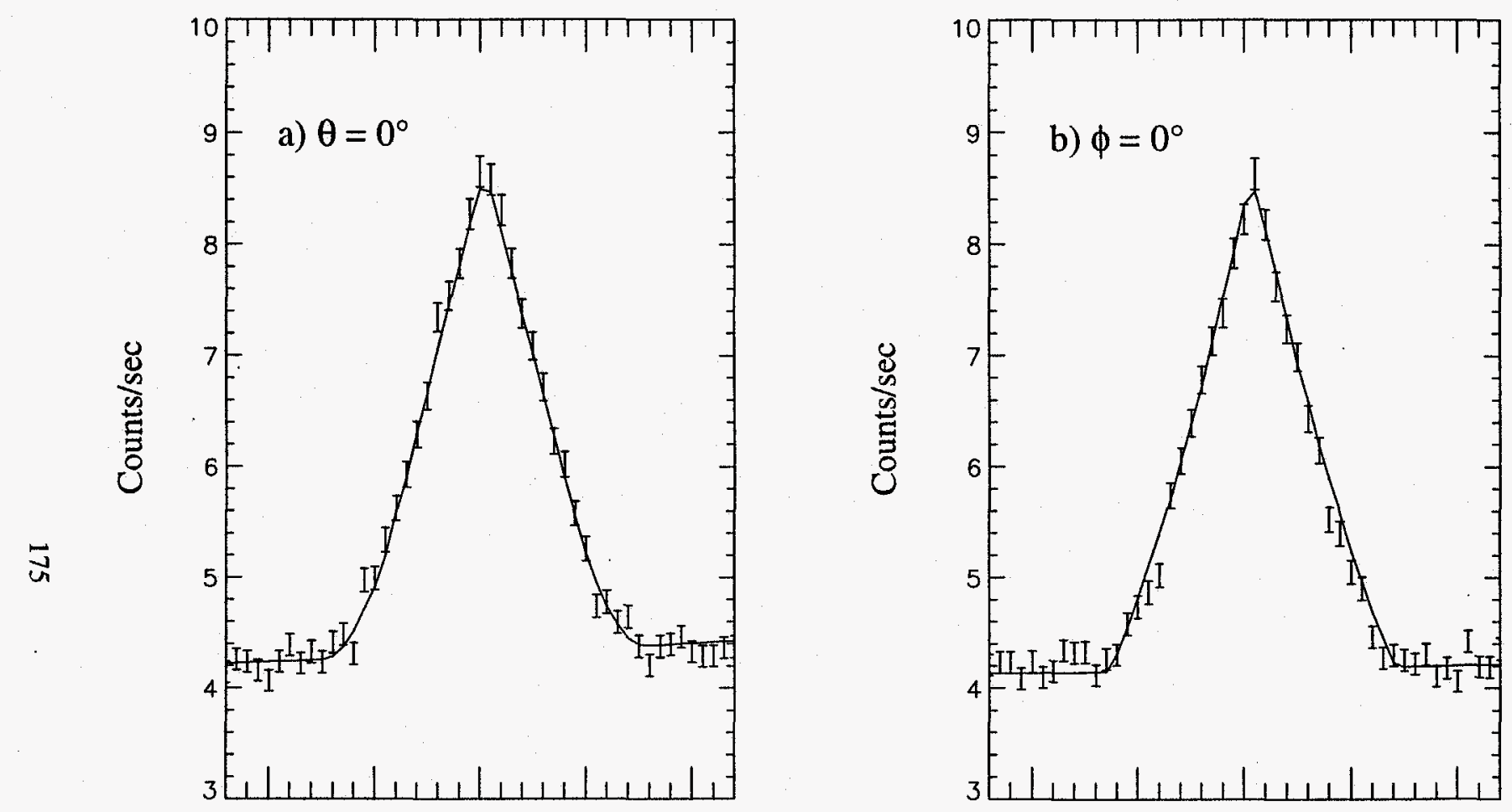

Fig. D.24 :

Scans in a) $\phi$ (yaw) and in b) $\theta$ (pitch) for unit:

Collimator: C-3

Segment: 2

Module: 21

Simulated response

function using parameters

$$
(\phi),(\theta)
$$

FWHM: $1.22^{\circ}, 1.14^{\circ}$

Centers: $0.040^{\circ}, 0.073^{\circ}$
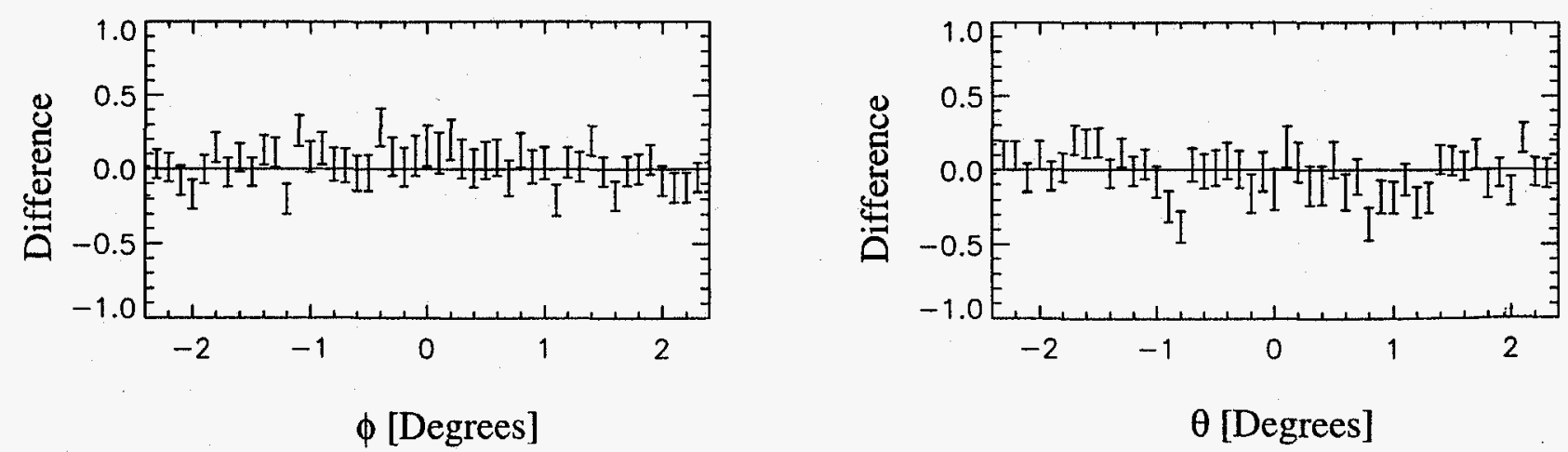

from fit to data are shown in solid curve. Difference between data and simulation shown in lower curves. 

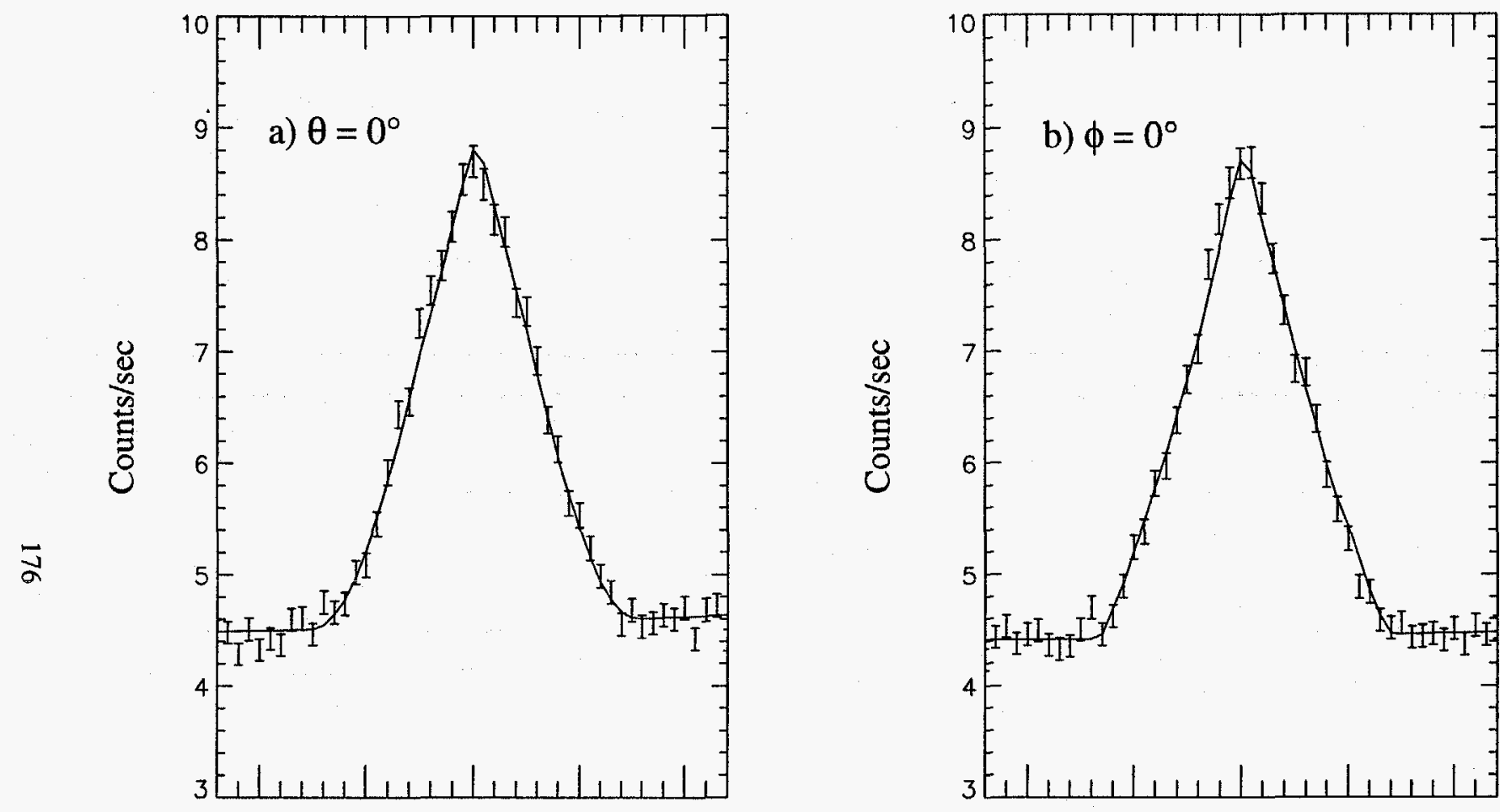

Fig. D.25 :

Scans in a) $\phi$ (yaw) and in b) $\theta$ (pitch) for unit:

Collimator: C-3

Segment: 2

Module: 25

Simulated response

function using parameters

$$
(\phi),(\theta)
$$

FWHM: $1.23^{\circ}, 1.19^{\circ}$

Centers: $0.024^{\circ}, 0.032^{\circ}$
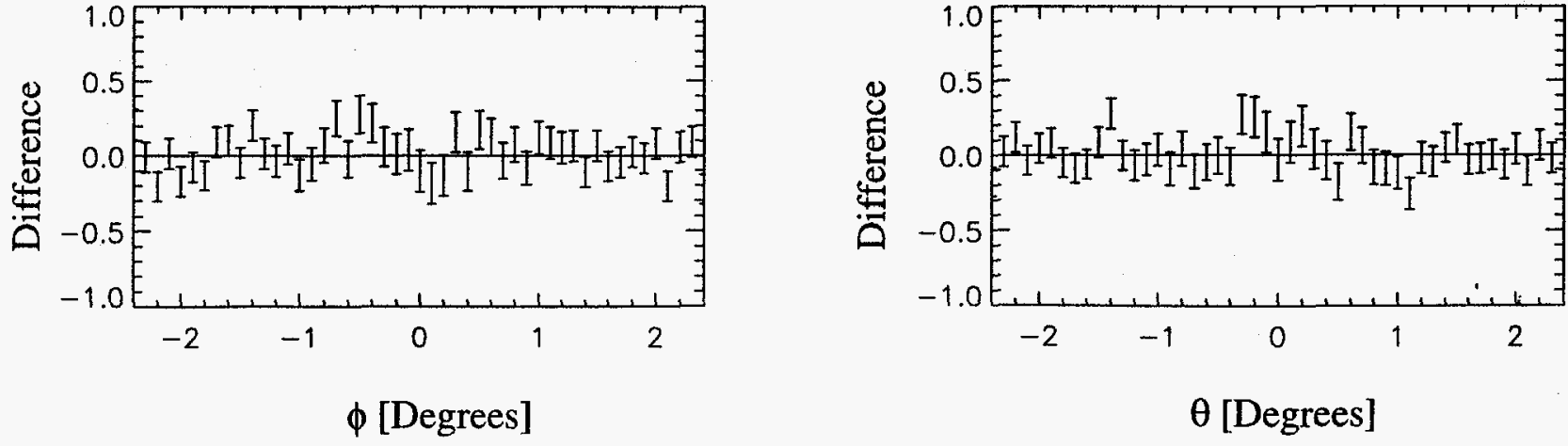

from fit to data are shown in solid curve. Difference between data and simulation shown in lower curves. 
Appendix E

\section{Charged Particle Backgrounds in the USA Detector}

\section{E.1 Introduction}

The time available for X-ray observations in Earth-orbit is limited by regions of high charged particle flux, where the background counting rates are too high for X-ray detectors to operate. An estimate is made of this available time or uptime for the USA detector. Phenomenological models are first utilized to estimate the flux of charged particles at USA's altitude for a given longitude and latitude. The charged particles are made incident on the USA detector by Monte Carlo simulation and then propagated through the various materials to determine the observed counting rate. The USA orbit is then simulated to estimate the fraction of orbital time with counting rates below a detector cutoff.

\section{E.2 Monte Carlo Simulation}

The active X-ray detecting volume of the USA detector is defined by its proportional chamber. There are two detector units with identical proportional chambers. All simulations and counting rate estimates will refer to one detector unit. The rate of charged particles penetrating into the proportional chamber is given by 


$$
\frac{d N}{d t}=\sum_{n=e^{-}, p} \int d E \int d \Omega \oint_{S} d a\left(\frac{d^{2} \Phi}{d \Omega d E}\right)_{n} t_{n, S}(\mathbf{r}, E)
$$

where the surface integral is performed over all faces of the detector unit, $\left(d^{2} \Phi / d \Omega d E\right)_{n}$ is the differential flux [particles/( $\left.\left.\left.\mathrm{cm}^{2} \cdot \mathrm{ster} \cdot \mathrm{sec}\right) / \mathrm{MeV}\right)\right]$ and $t_{n, S}(\mathbf{r}, E)$ is the probability (0 or 1) that a charged particle incident on surface $S$ at position and direction $\mathbf{r}$ and kinetic energy $E$ will be recorded in the USA proportional chamber

The charged particle fluxes were determined from phenomenological models that provided omnidirectional estimates

$$
\frac{d \Phi}{d E}=4 \pi \frac{d^{2} \Phi}{d \Omega d E}
$$

which factor out of the solid angle and surface integrals in [E.1]. The closed surface integral in [E.1] can be rewritten as the sum of surface integrals over all the faces of the detector unit $S_{i}$

$$
\oint_{S} d a=\sum_{S_{i}} \int_{S_{i}} d a=\sum_{S_{i}} A_{i} \int_{S_{i}} \frac{d a}{A_{i}}
$$

Finally, defining the total transmission probability for particles of type $n$ incident on face $S_{i}$ with kinetic energy $\mathrm{E}$ as

$$
T_{n, S_{i}}(E) \equiv \int \frac{d \Omega}{4 \pi} \int_{S_{i}} \frac{d a}{A_{i}} t_{n, S_{i}}(\mathbf{r}, E)
$$

and substituting it with [E.2] and [E.3] into [E.1] gives the final expression for the charged particle rate observed in the proportional chamber of a USA detector unit,

$$
\frac{d N}{d t}=\sum_{n=e^{-}, p} \sum_{S_{i}} A_{i} \int d E\left(\frac{d \Phi}{d E}\right)_{n} T_{n, S_{i}}(E)
$$

The total transmission probabilities were evaluated by approximating the integrals in expression [E.4] by sums 


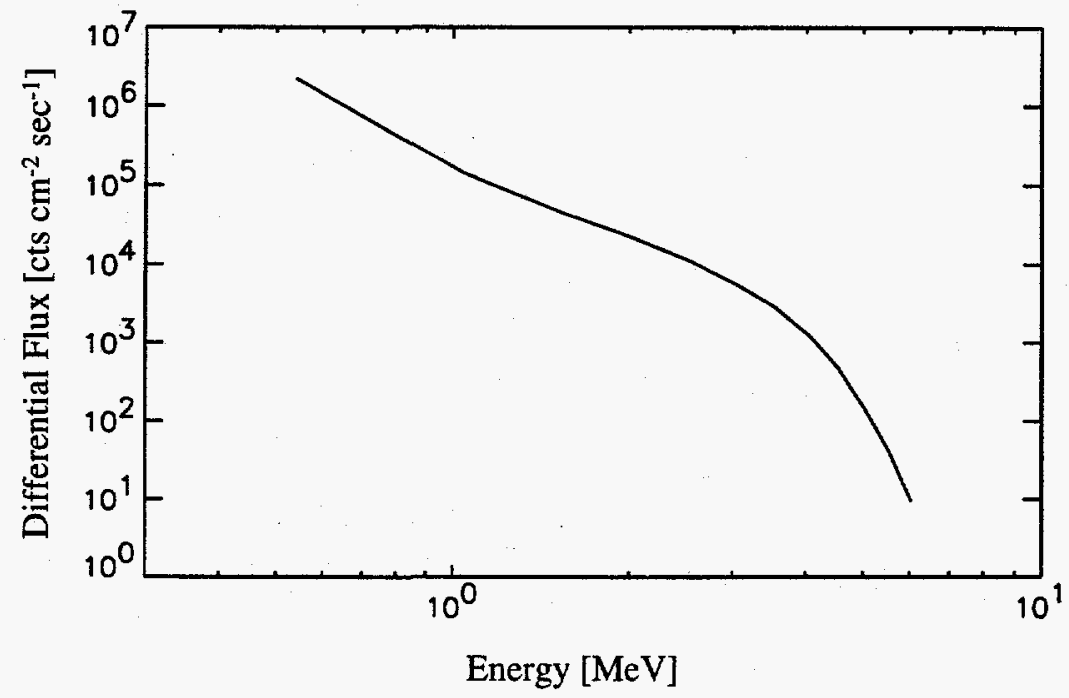

Fig. E.I Typical differential flux distribution of electrons trapped in the van Allen radiation belts. Model: AE8 (NSSDC) at an altitude of $834 \mathrm{~km}$.

$$
T_{n, S_{i}}(E)=\sum \frac{\Delta \Omega}{4 \pi} \sum \frac{\Delta a}{A_{i}} t_{n, S_{i}}(\mathbf{r}, E)
$$

and evaluating the sums by method of Monte Carlo simulation. Charged particles at kinetic energy $E$ were generated at random incident positions and solid angles onto one of the detector unit faces $S_{i}$. The trajectory of each particle was then propagated through the detector materials. If the trajectory penetrated the proportional chamber volume and the energy lost traversing the materials was less than the particle's initial kinetic energy then that particle was recorded as an event. The total transmission probability is the fraction of incident particles that are recorded as events in the proportional chamber.

\section{E.2.1 Sources of Charged Particle Background}

The charged particle background originates primarily from the solar wind and the galaxy. The Earth's magnetosphere interacts with the solar wind by trapping solar particles (electrons and protons) in doughnut-shaped van Allen radiation belts. At a given altitude, these torroidal belts intersect a sphere by forming two rings or bands at high and low latitudes with high charged particle flux. Another region of high charged particle flux is known as the South Atlantic Anomaly (SAA), an elliptical region centered 
above Brazil, spanning roughly $130^{\circ}$ in longitude and $50^{\circ}$ in latitude (see Figure E.4). In the SAA, the magnetic flux is anomalously low, allowing particles from the inner belt to brush the top of the atmosphere.

Phenomenological models ${ }^{1}$ of the flux of trapped particles have been developed by the U.S. National Space Science Data Center (NSSDC) at NASA's Goddard Space Flight Center. They are based on almost all available satellite data. The models, AP8 for protons $^{2}$ and AE8 for electrons ${ }^{3}$ provide omnidirectional estimates of differential flux down to 1 particle $\mathrm{cm}^{-2} \mathrm{sec}^{-1}$ at an energy spectrum ranging from 0.1 to $400 \mathrm{MeV}$ for the protons and from 0.04 to $7 \mathrm{MeV}$ for the electrons. A typical differential flux distribution is shown in Figure E.1 for electrons in the radiation belts. The electron flux dominates over the proton flux by several orders of magnitude.

Galactic cosmic rays are focused at the Earth's poles where the density of magnetic field lines is the highest. Developed at the Naval Research Laboratory (NRL), the Cosmic Ray Effects of Microelectronics (CREME) model $^{4}$ was used to estimate the differential flux from cosmic rays. Consisting primarily of protons, the CREME model estimates the differential flux at an energy spectrum ranging from $10 \mathrm{MeV}$ to 30 $\mathrm{GeV}$ for various interplanetary and magnetic weather conditions. Figure E.2 shows a typical differential flux distribution for cosmic rays near the poles.

\section{E.2.2 Detector Physics}

Once the flux of charged particles incident on the USA detector is estimated, the task of translating that flux into an actual detector count rate is left to the detector physics simulation. Charged particles that penetrate into the detector's chamber volume must traverse either the collimator or the proportional chamber walls. The trajectory of the incident particle must project into the chamber volume with more incident energy than is

\footnotetext{
${ }^{1}$ Bilitza, D. (1988), "Radbelt, Trapped Radiation Models", NSSDC ID:PT-14A.

${ }^{2}$ Sawyer, D. W. \& Vette, J. I. (1976), “AP-8 Trapped Proton Environment for Solar Maximum and Minimum", NSDDC/WDC-A-R\&S 76-06.

${ }^{3}$ Teague, M. J., Chan, K. W., \& Vette, J. I. (1976), "AE-8: A Model Environment of Trapped Electrons for Solar Maxiumum", NSSDC/WDC-A-R\&S 76-04.

${ }^{4}$ Adams, J.H. (1986), "Cosmic Ray Effects on Microelectronics, Part IV", NRL Report 5901.
} 


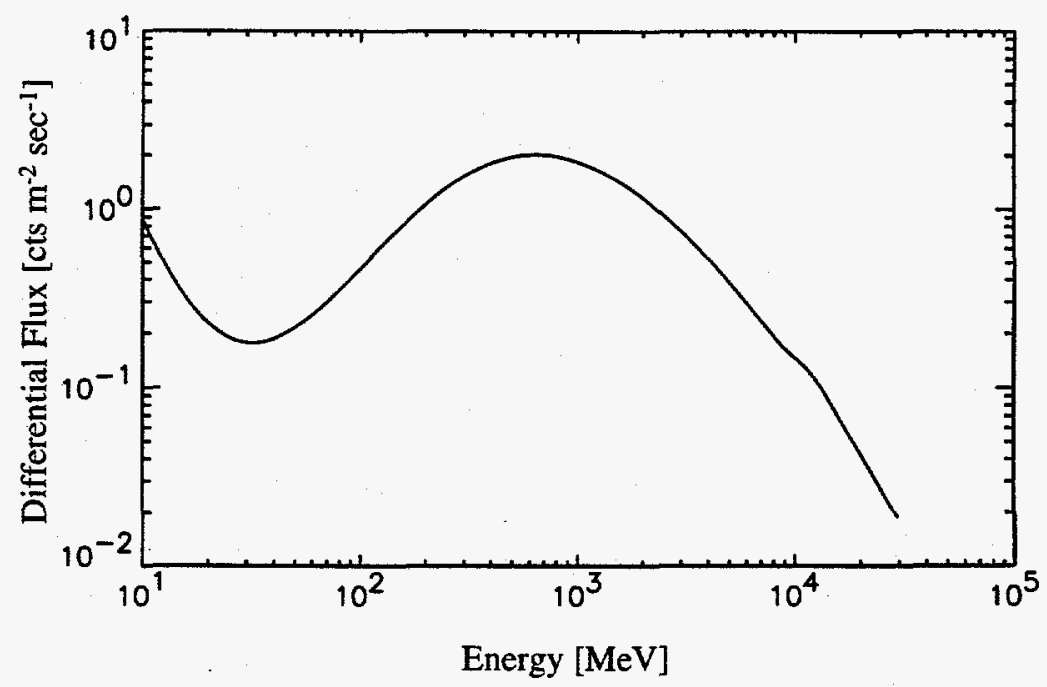

Fig. E.2 Typical differential flux distribution of galactic cosmic rays. Model: CREME (NRL) at an altitude of $834 \mathrm{~km}$.

lost while traversing these materials.

Most of the energy lost is due to ionization. The $d E / d x$ curves for this process were determined using the Berger-Seltzer formula ${ }^{5}$ for the electrons and the restricted energy loss formula ${ }^{6}$, a modification of the Bethe-Bloch equation, for the protons. Although both of these formulae allow for the explicit generation of delta rays, the energy loss for both particle species was treated as continuous.

Special treatment was given to the calculation of energy loss through the collimator. A uniform effective density was chosen to represent the collimator honeycomb material. This density was the ratio of the total collimator mass divided by the total collimator volume. However, particles incident on the top face with angles less than $0.75^{\circ}$ from the normal were passed through the collimator without interacting with the material.

\footnotetext{
${ }^{5}$ Berger, M. J. \& Selzer, S. M. (1964), NASA SP 3012.

${ }^{6}$ Particle Data Group (1992), Review of Particle Properties, p. III.14.
} 


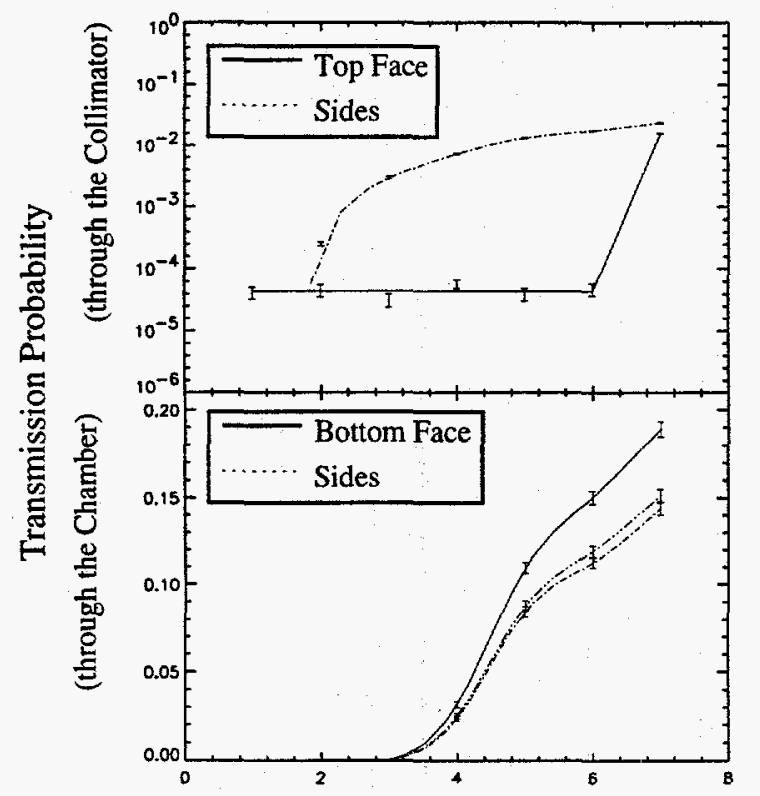

Electron Energy [MeV]

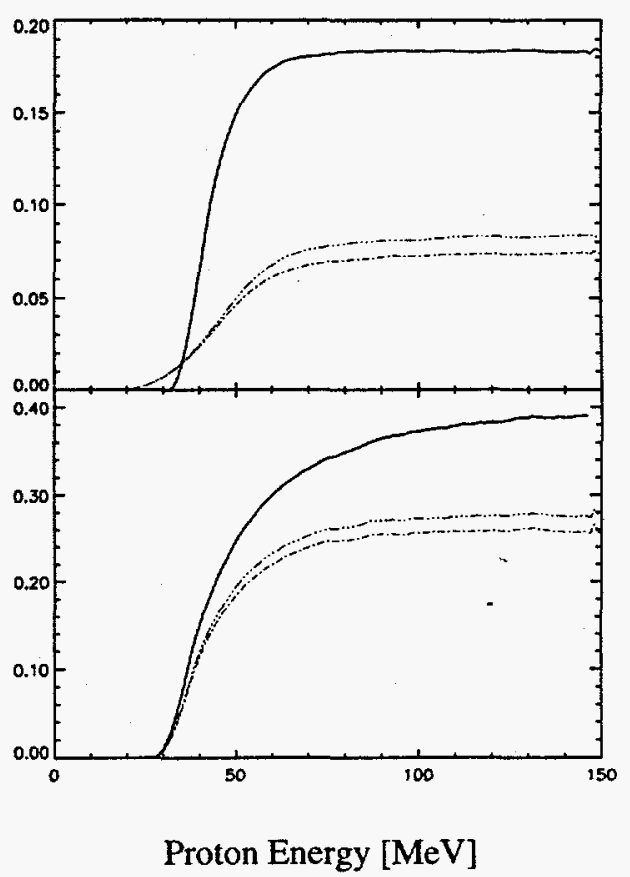

Proton Energy $[\mathrm{MeV}]$

Fig. E.3 Transmission probabilities through the various faces of the collimator and the proportional chamber walls. The top and bottom rows are transmissions through the collimator and proportional chamber, respectively. The left and right columns specifies the particle species, electrons and protons, respectively.

Applying the simulation as previously described in conjunction with the $d E / d x$ curves, the total transmission probabilities, [E.6] were determined through each of the 3 different faces of the collimator and the proportional chamber. Figure E.3 summarizes these transmission probabilities covering energy ranges with significant differential flux of electrons or protons.

\section{E.3 Results}

\section{E.3.1 Charged Particle Background Counting Rates}

Combining the transmission probabilities with the differential flux of electrons and protons, the charged particle background counting rates, [E.5] were estimated for all longitudes and latitudes in $1^{\circ}$ increments at the altitude of the USA detector, $834 \mathrm{~km}$. Figures E.4, E.5 and E.6 shows the contour maps of counting rates due to charged 


\begin{tabular}{|c|c|}
\hline Region & $\begin{array}{c}\text { Average Expected } \\
\text { Background Counting Rate } \\
{\left[\text { counts sec }^{-1} \text { ] }\right.}\end{array}$ \\
\hline Equatorial & $\sim 1.5 \times 10^{3}$ \\
\hline Polar & $\sim 6 \times 10^{3}$ \\
\hline Radiation Belts & $\sim 2 \times 10^{5}$ \\
\hline South Atlantic Anomaly & $\sim 2 \times 10^{6}$ \\
\hline
\end{tabular}

Table E.1 Average expected counting rate in a USA detector unit from all estimated particle backgrounds. The equatorial region lies between the two radiation belt bands and outside the SAA.

particles trapped by the Earth's magnetosphere, galactic cosmic rays and all charged particles, respectively. As Figure E.4 shows, rates in the two radiation belt bands and the South Atlantic Anomaly dominate the trapped charged particle background. For the galactic cosmic rays, Figure 3.5 shows a smooth distribution of counting rates rising to maxima at the polar regions. The averages for various regions of Figure E.6 are summarized in Table E.1.

\section{E.3.2 X-ray Observation Uptime}

A USA detector unit shuts down when the counting rate in its proportional counter exceeds $10^{4}$ counts $\sec ^{-1}$. The fraction of USA's orbit spent with background rates below this cutoff is defined as the uptime. To calculate this uptime, the orbit of the ARGOS satellite was simulated with an expected orbital inclination of $98.7^{\circ}$ and a period of 102 minutes. The Earth's rotation was also included in the simulation. The uptime was averaged over 1800 orbits or 127 days using contour maps of the total charged particle counting rates as the one shown in Figure E.6. Table E.2 summarizes the uptimes calculated from this simulation. 


\begin{tabular}{|c|c|c|c|}
\hline Solar Epoch & $\begin{array}{c}\text { Magnetic Weather } \\
\text { Condition }\end{array}$ & $\begin{array}{c}\text { Interplanetary } \\
\text { Weather Index }\end{array}$ & \% Uptime \\
\hline \multirow{2}{*}{ Maximum } & \multirow{2}{*}{ Stormy } & 1 & 61.8 \\
\cline { 3 - 4 } & & 2 & 60.9 \\
\cline { 3 - 4 } & & 3 & 46.2 \\
\cline { 2 - 4 } & & 4 & 46.2 \\
\hline \multirow{2}{*}{ Minimum } & \multirow{3}{*}{ Stormy } & 1 & 61.1 \\
\cline { 3 - 4 } & & 2 & 60.7 \\
\cline { 3 - 4 } & & 3 & 45.8 \\
\cline { 3 - 4 } & & 4 & 45.8 \\
\hline
\end{tabular}

Table E.2 The percentage of uptime below a $10^{4}$ counts $\sec ^{-1}$ cutoff for several scenarios in the trapped charged particle and cosmic ray models. The interplanetary weather index is defined as follows: 1 galactic cosmic rays only (quiet period), 2 - worst-case galactic cosmic rays allowing for uncertainty in flux data and solar activity, 3 - peak ordinary flare flux and mean composition and 4 - peak $10 \%$ worst-case flare flux and mean composition. 


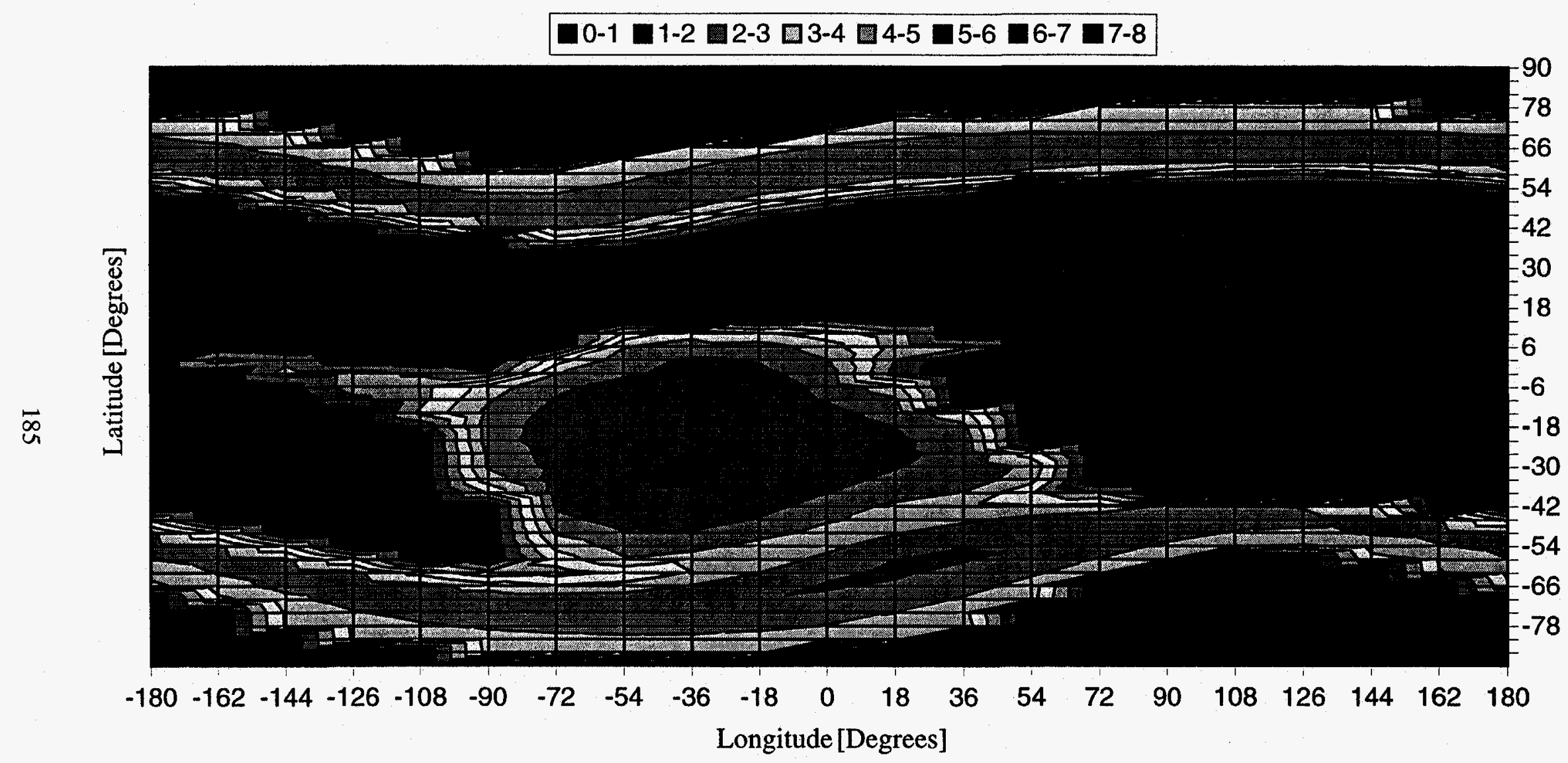

Fig. E.4 Contour map of the expected background rates in one USA detector unit due to charged particles trapped by the Earth's magnetosphere. The numbers in the legend represents the $\log _{10}$ (Counting rates [counts $\left.\mathrm{sec}^{-1}\right]$ ). The two bands due to the radiation belts and the South Atlantic Anomaly are the most notable features. 


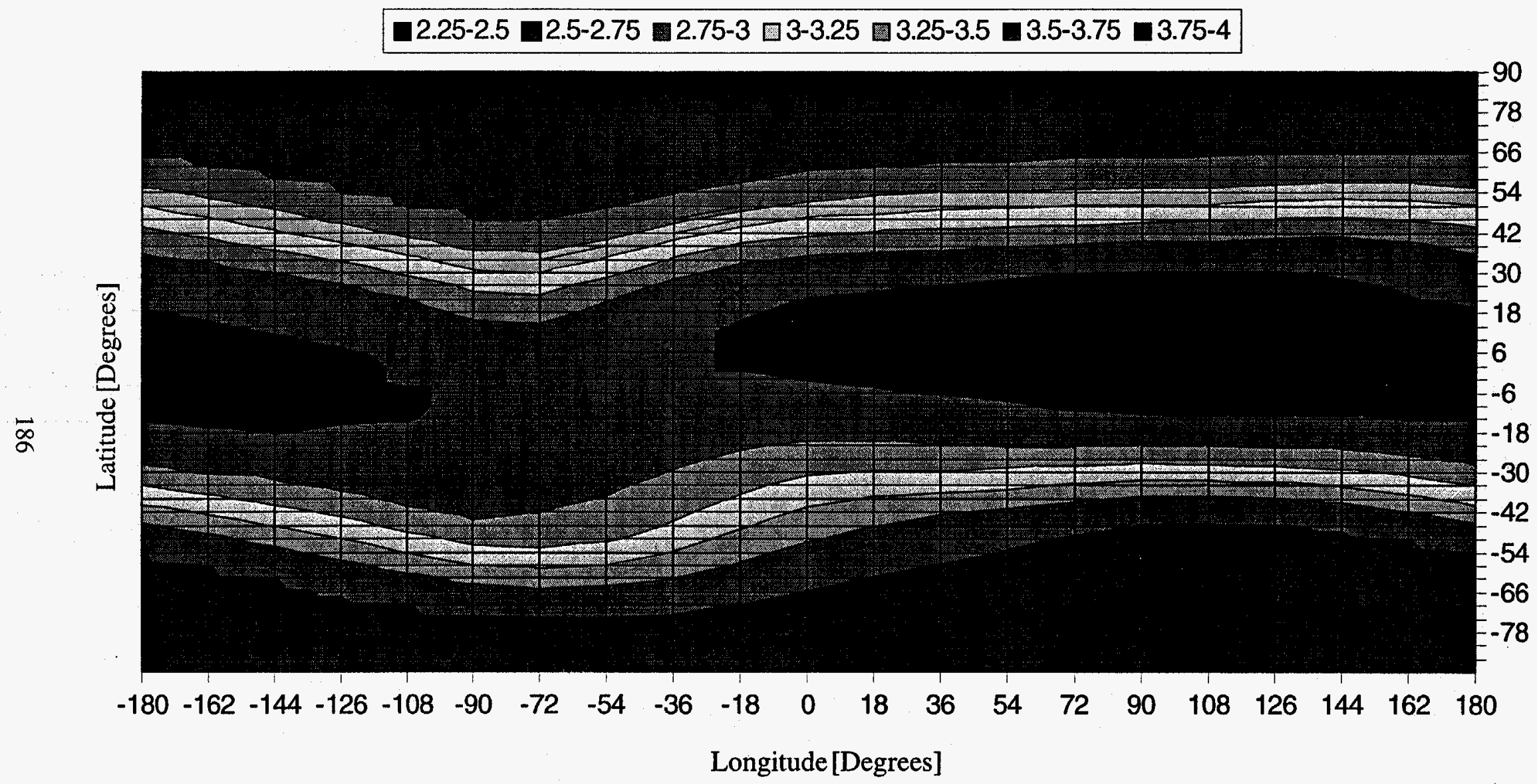

Fig. E.5 Contour map of the expected background rates in one USA detector unit due to galactic cosmic rays. The numbers in the legend represents the $\log _{10}$ (Counting rates [countssec $\left.\left.{ }^{-1}\right]\right)$. The rates are highest at the poles where the density of the Earth's magnetic field lines is the highest. 


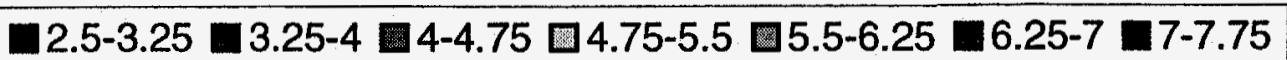

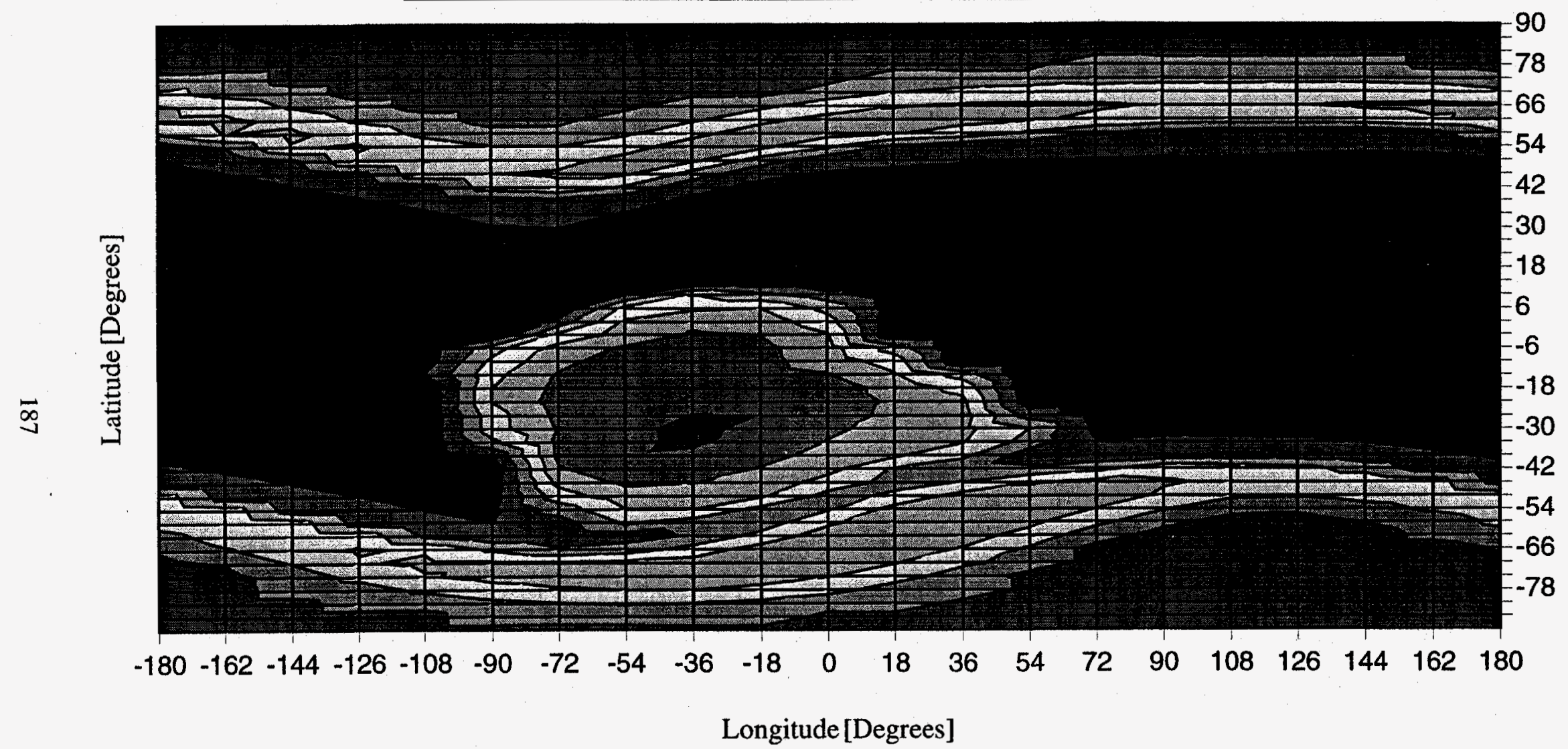

Fig. E.6 Contour map of the expected background rates in one USA detector unit due to all charged particles. The numbers in the legend represents the $\log _{10}$ (Counting rates [counts sec ${ }^{-1}$ ]). 
I certify that I have read this dissertation and that in my opinion it is fully adequate, in scope and quality, as a dissertation for the degree of Doctor of Philosophy.

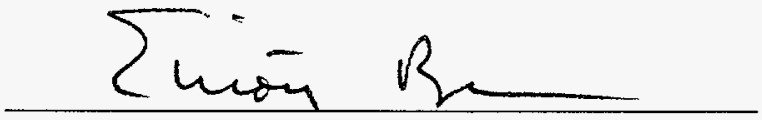

(Elliott Bloom) Principal Advisor

I certify that I have read this dissertation and that in my opinion it is fully adequate, in scope and quality, as a dissertation for the degree of Doctor of Philosophy.

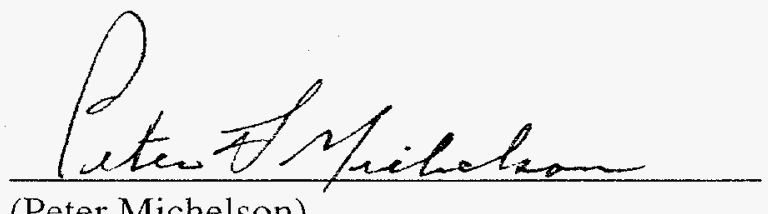

(Peter Michelson)

I certify that I have read this dissertation and that in my opinion it is fully adequate, in scope and quality, as a dissertation for the degree of Doctor of Philosophy.

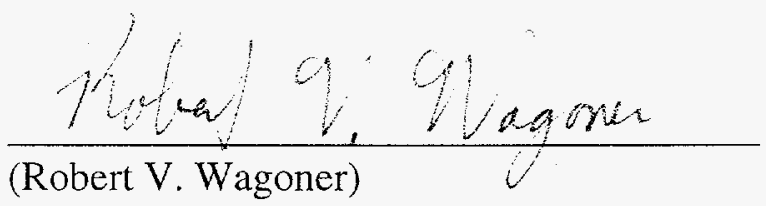

Approved for the University Committee on Graduate Studies:

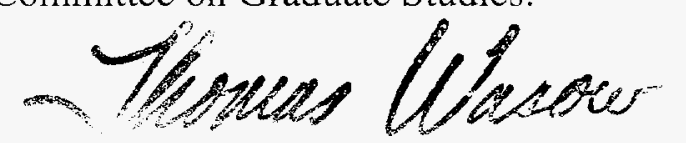

